\title{
Estimation of Monthly Water Yields and Flows for 1951-2012 for the United States Portion of the Great Lakes Basin with AFINCH
}

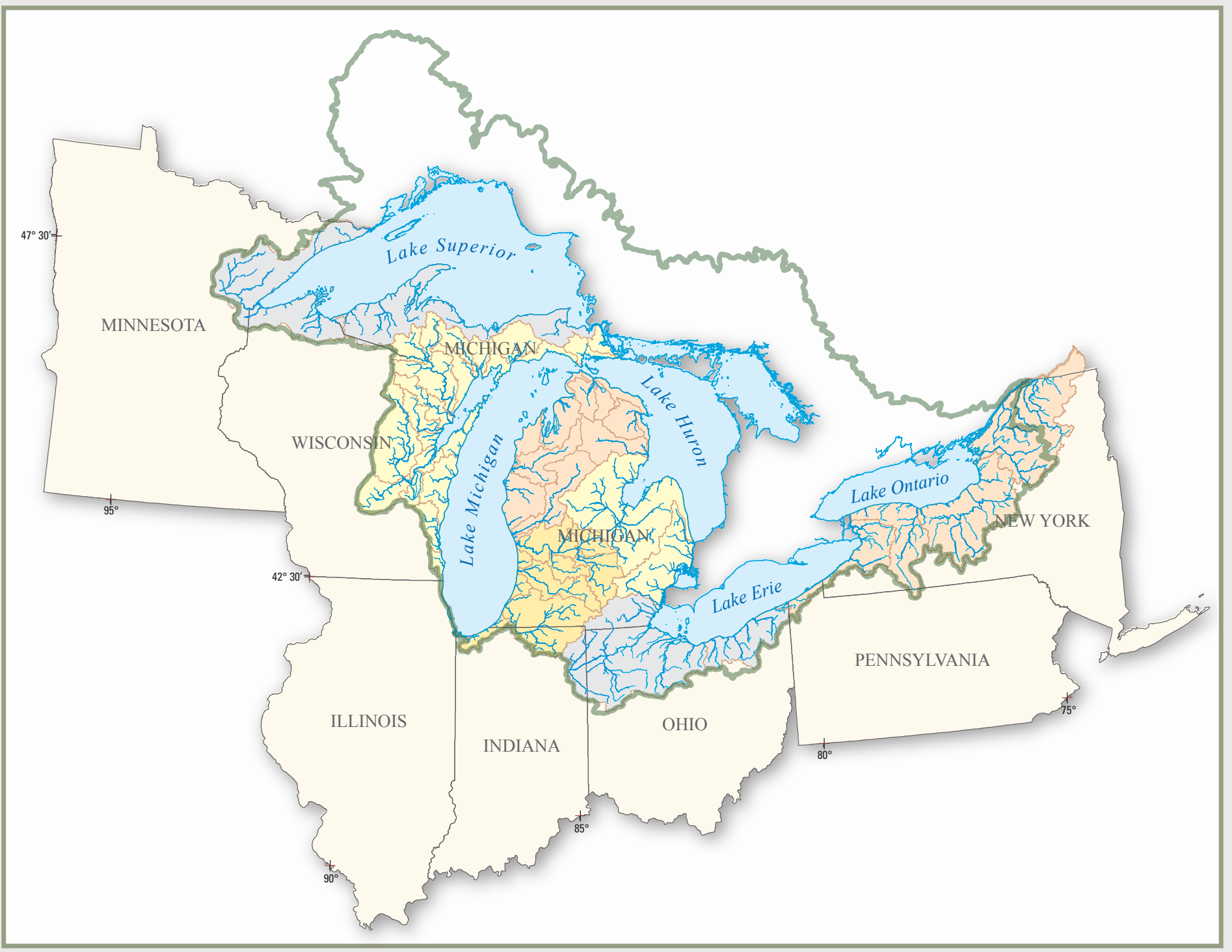

Scientific Investigations Report 2014-5192 
Cover image. The United States portion of the Great Lakes Basin. 


\section{Estimation of Monthly Water Yields and Flows for 1951-2012 for the United States Portion of the Great Lakes Basin with AFINCH}

By Carol L. Luukkonen, David J. Holtschlag, Howard W. Reeves, C.J. Hoard, and Lori M. Fuller

Scientific Investigations Report 2014-5192 


\title{
U.S. Department of the Interior SALLY JEWELL, Secretary
}

\section{U.S. Geological Survey Suzette M. Kimball, Acting Director}

\author{
U.S. Geological Survey, Reston, Virginia: 2015
}

For more information on the USGS - the Federal source for science about the Earth, its natural and living resources, natural hazards, and the environment, visit http://www.usgs.gov or call 1-888-ASK-USGS.

For an overview of USGS information products, including maps, imagery, and publications, visit http://www.usgs.gov/pubprod

To order this and other USGS information products, visit http://store.usgs.gov

Any use of trade, firm, or product names is for descriptive purposes only and does not imply endorsement by the U.S. Government.

Although this information product, for the most part, is in the public domain, it also may contain copyrighted materials as noted in the text. Permission to reproduce copyrighted items must be secured from the copyright owner.

Suggested citation:

Luukkonen, C.L., Holtschlag, D.J., Reeves, H.W., Hoard, C.J., and Fuller, L.M., 2015, Estimation of monthly water yields and flows for 1951-2012 for the United States portion of the Great Lakes Basin with AFINCH: U.S. Geological Survey Scientific Investigations Report 2014-5192, 83 p, http://dx.doi.org/10.3133/sir20145192.

ISSN 2328-0328 (online) 


\section{Contents}

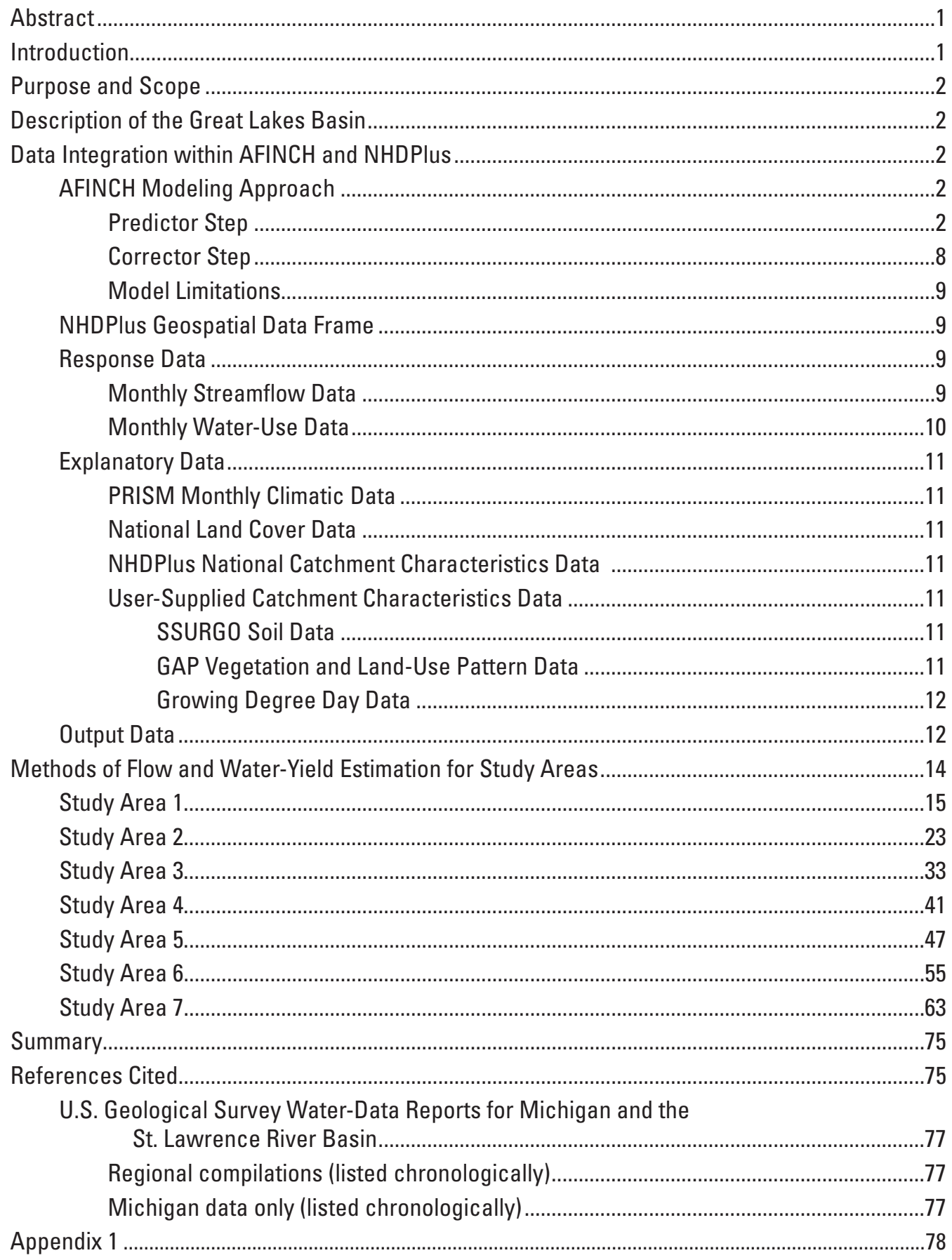




\section{Figures}

1. Map showing the hydrologic subregions and cataloguing units in the U.S. Great Lakes Basin .

2. Map showing the distribution of estimated water yields for August averaged over 1951-2012.

3. Time-series plot of monthly flows at flowline 11959440, streamgaging station 04058000, Middle Branch Escanaba River near Ishpeming, Michigan

4. Map showing the location of study area 1 (hydrologic subregions 0401 and 0402) in the U.S. Great Lakes Basin

5. Graph showing the t-value-indexed colors corresponding to each explanatory variable in the monthly regression equation for estimating water yield for study area 1 (hydrologic subregions 0401 and 0402) in the U.S. Great Lakes Basin

6. Map showing the location of study area 2 (hydrologic subregion 0403 and cataloguing units $04040002,04040003,04060106,04060107,04070001$, and 04070002) in the U.S. Great Lakes Basin

7. Graph showing the t-value-indexed colors corresponding to each explanatory variable in the monthly regression equation for estimating water yield for study area 2 (hydrologic subregion 0403 and cataloguing units $04040002,04040003,04060106,04060107,04070001$, and 04070002) in the U.S. Great Lakes Basin

8. Map showing the location of study area 3 (hydrologic subregion 0405 and cataloguing unit 04040001) in the U.S. Great Lakes Basin

9. Graph showing the t-value-indexed colors corresponding to each explanatory variable in the monthly regression equation for estimating water yield for study area 3 (hydrologic subregion 0405 and cataloguing unit 04040001) in the U.S. Great Lakes Basin

10. Map showing the location of study area 4 (cataloguing units 04060101, 04060102, 04060103, 04060104, 04060105, 04070003, 04070004, 04070005, 04070006, and 04070007) in the U.S. Great Lakes Basin

11. Graph showing the t-value-indexed colors corresponding to each explanatory variable in the monthly regression equation for estimating water yield for study area 4 (cataloguing units 04060101, 04060102, 04060103, 04060104, 04060105, 04070003, 04070004, 04070005, 04070006, and 04070007) in the U.S. Great Lakes Basin

12. Map showing the location of study area 5 (hydrologic subregions 0408 and 0409) in the U.S. Great Lakes Basin

13. Graph showing the t-value-indexed colors corresponding to each explanatory variable in the monthly regression equation for estimating water yield for study area 5 (hydrologic subregions 0408 and 0409) in the U.S. Great Lakes Basin

14. Map showing the location of study area 6 (hydrologic subregions 0410 and 0411) in the U.S. Great Lakes Basin

15. Graph showing the t-value-indexed colors corresponding to each explanatory variable in the monthly regression equation for estimating water yield for study area 6 (hydrologic subregions 0410 and 0411) in the U.S. Great Lakes Basin 


\section{Figures (continued)}

16. Map showing the location of study area 7 (hydrologic subregions 0412 , 0413, 0414, and 0415) in the U.S. Great Lakes Basin

17. Map showing the t-value-indexed colors corresponding to each explanatory variable in the monthly regression equation for estimating water yield for study area 7 (hydrologic subregions 0412, 0413, 0414, and 0415) in the U.S. Great Lakes Basin

\section{Tables}

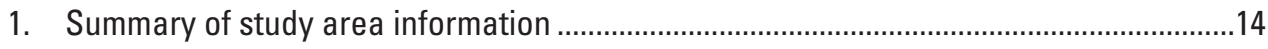

2. Streamgaging stations considered for analysis of study area 1 (hydrologic subregions 0401 and 0402) in the U.S. Great Lakes Basin, by water year........................16

3. Selected explanatory variables for each study area.......................................................

4. Regression statistics and explanatory variables used in the AFINCH analysis of flows in study area 1 (hydrologic subregions 0401 and 0402), in the U.S. Great Lakes Basin, by month.

5. Streamgaging stations considered for analysis of study area 2 (hydrologic subregion 0403 and cataloguing units 04040002, 04040003, 04060106, 04060107, 04070001, and 04070002) in the U.S. Great Lakes Basin, by water year.

6. Regression statistics and explanatory variables used in the AFINCH analysis for study area 2 (hydrologic subregion 0403 and cataloguing units 04040002, 04040003, 04060106, 04060107, 04070001, and 04070002) in the U.S. Great Lakes Basin, by month

7. Streamgaging stations considered for analysis of study area 3 (hydrologic subregion 0405 and cataloguing unit 04040001) in the U.S. Great Lakes Basin, by water year.

8. Regression statistics and explanatory variables used in the AFINCH analysis for study area 3 (hydrologic subregion 0405 and cataloguing unit 04040001) in the U.S. Great Lakes Basin, by month

9. Streamgaging stations considered for analysis of study area 4 (cataloguing units 04060101, 04060102, 04060103, 04060104, 04060105, 04070003, 04070004, 04070005, 04070006, and 04070007) in the

U.S. Great Lakes Basin, by water year.

10. Regression statistics and explanatory variables used in the AFINCH analysis for study area 4 (cataloguing units 04060101, 04060102, 04060103, 04060104, 04060105, 04070003, 04070004, 04070005, 04070006, and 04070007) in the U.S. Great Lakes Basin, by month.

11. Streamgaging stations considered for analysis of study area 5 (hydrologic subregions 0408 and 0409) in the U.S. Great Lakes Basin, by water year.

12. Regression statistics and explanatory variables used in the AFINCH analysis for study area 5 (hydrologic subregions 0408 and 0409) in the U.S. Great Lakes Basin, by month. 


\section{Tables (continued)}

13. Streamgaging stations considered for analysis of study area 6

(hydrologic subregions 0410 and 0411) in the U.S. Great Lakes Basin,

by water year.

14. Regression statistics and explanatory variables used in the AFINCH analysis for study area 6 (hydrologic subregions 0410 and 0411) in the U.S. Great Lakes Basin, by month

15. Streamgaging stations considered for analysis of study area 7 (hydrologic subregions 0412, 0413, 0414, and 0415) in the U.S. Great Lakes Basin, by water year.

16. Regression statistics used in the AFINCH analysis for study area 7 (hydrologic subregions 0412, 0413, 0414, and 0415) in the

U.S. Great Lakes Basin, by month

\section{Appendix Tables}

1-1. Revisions to NHDPlus flowline value-added attribute (VAA) information ........................78

1-2. Description of classes in the 2006 National Land Cover Database. ...............................82

1-3. Description of soil groups in the Soil Survey Geography (SSURGO) database...............83

1-4. Description of variables from Aquatic Gap Analysis Program (GAP) used in the analysis. 


\section{Conversion Factors and Datums}

\begin{tabular}{lcl}
\hline \multicolumn{1}{c}{ Multiply } & By & \multicolumn{1}{c}{ To obtain } \\
\hline & Length & \\
\hline mile $(\mathrm{mi})$ & 1.609 & kilometer $(\mathrm{km})$ \\
kilometer $(\mathrm{km})$ & 0.6214 & mile $(\mathrm{mi})$ \\
\hline & Area & \\
\hline square mile $\left(\mathrm{mi}^{2}\right)$ & 259.0 & hectare $(\mathrm{ha})$ \\
square mile $\left(\mathrm{mi}^{2}\right)$ & 2.590 & square kilometer $\left(\mathrm{km}^{2}\right)$ \\
\hline & Volume & \\
\hline cubic mile $\left(\mathrm{mi}^{3}\right)$ & 4.168 & cubic kilometer $\left(\mathrm{km}^{3}\right)$ \\
\hline & Flow rate & \\
\hline cubic foot per second $\left(\mathrm{ft}^{3} / \mathrm{s}\right)$ & 0.02832 & cubic meter per second $\left(\mathrm{m}^{3} / \mathrm{s}\right)$ \\
\hline
\end{tabular}

Temperature in degrees Celsius $\left({ }^{\circ} \mathrm{C}\right)$ may be converted to degrees Fahrenheit $\left({ }^{\circ} \mathrm{F}\right)$ as follows:

${ }^{\circ} \mathrm{F}=\left(1.8 x^{\circ} \mathrm{C}\right)+32$

Vertical coordinate information is referenced to the North Geodetic Vertical Datum of 1929

(NGVD 29).

Horizontal coordinate information is referenced to the North American Datum of 1983 (NAD 83).

Altitude, as used in this report, refers to distance above the vertical datum.

A water year is the 12-month period October 1 through September 30. The water year is designated by the calendar year in which it ends and which includes 9 of the 12 months. Thus, the year ending September 30, 2002, is called the "2002 water year."

\section{Abbreviations}

$\begin{array}{ll}\text { AFINCH } & \text { Analysis of Flows In Networks of CHannels } \\ \text { GAP } & \text { USGS National Gap Analysis Program } \\ \text { HUC } & \text { hydrologic unit code } \\ \text { NED } & \text { National Elevation Dataset } \\ \text { NHD } & \text { National Hydrography Dataset } \\ \text { NLCD } & \text { National Land Cover Data } \\ \text { NPDES } & \text { National Pollutant Discharge Elimination System } \\ \text { OLS } & \text { ordinary least square } \\ \text { POA } & \text { period of analysis } \\ \text { PRISM } & \text { Parameter-elevation Regressions on Independent Slopes Model } \\ \text { R } & \text { coefficient of determination } \\ \text { RMSE } & \text { root mean square error } \\ \text { SSURGO } & \text { Soil Survey Geography } \\ \text { USGS } & \text { U.S. Geological Survey } \\ \text { VAA } & \text { value-added attributes }\end{array}$





\title{
Estimation of Monthly Water Yields and Flows for 1951-2012 for the United States Portion of the Great Lakes Basin with AFINCH
}

\author{
By Carol L. Luukkonen, David J. Holtschlag, Howard W. Reeves, C.J. Hoard, and Lori M. Fuller
}

\section{Abstract}

Monthly water yields from 105,829 catchments and corresponding flows in 107,691 stream segments were estimated for water years 1951-2012 in the Great Lakes Basin in the United States. Both sets of estimates were computed by using the Analysis of Flows In Networks of CHannels (AFINCH) application within the NHDPlus geospatial data framework. AFINCH provides an environment to develop constrained regression models to integrate monthly streamflow and wateruse data with monthly climatic data and fixed basin characteristics data available within NHDPlus or supplied by the user. For this study, the U.S. Great Lakes Basin was partitioned into seven study areas by grouping selected hydrologic subregions and adjoining cataloguing units. This report documents the regression models and data used to estimate monthly water yields and flows in each study area. Estimates of monthly water yields and flows are presented in a Web-based mapper application. Monthly flow time series for individual stream segments can be retrieved from the Web application, The Great Lakes Restoration Initiative (GLRI) Mapper for Monthly Streamflow and Yield of Catchments using Analysis of Flows in Networks of CHannels (AFINCH), http://cida.usgs.gov/glri/afinch/, and used to approximate monthly flow-duration characteristics and to identify possible trends.

\section{Introduction}

A regionally consistent estimate of streamflow provides unified information across the U.S. Great Lakes Basin for restoration, assessment, management, and conservation of stream ecosystems. These estimated flows provide information for natural resource professionals and the general public to assess stream ecosystems across State jurisdictional boundaries and provide a framework that can be used to help enhance existing data networks. In addition, this information can be used to assess the status of stream ecosystems, to identify and prioritize locations to focus stream restoration efforts, to plan and manage game and non-game species, to protect and enhance aquatic systems for recreational use, to provide tools for assessing disturbance of aquatic ecosystems, to plan adaptive management strategies, and to aid in implementation of the Great Lakes-St. Lawrence River Basin Water Resources Compact (Council of Great Lakes Governors, 2005).

The Analysis of Flows In Networks of CHannels (AFINCH) application (Holtschlag, 2009) was applied to estimate monthly time series of yields and flows for water years 1951-2012 within the U.S. Great Lakes Basin (Hydrologic Region 04) (Seaber and others, 1987). The resulting long-term flow time series can be used to describe monthly flow-duration characteristics and to investigate trends at 107,691 stream segments in the U.S. Great Lakes Basin. AFINCH is an interactive program used to facilitate the estimation of time series of monthly water yields from catchments and flows in stream reaches defined within the 1:100,000-scale NHDPlus geospatial data framework (McKay and others, 2012). NHDPlus is an integrated suite of application-ready geospatial-data products that includes features from the National Hydrography Dataset (NHD; U.S. Geological Survey, 2009a), the National Elevation Dataset (NED; U.S. Geological Survey, 2009b), and the National Watershed Boundary Dataset (U.S. Department of Agriculture-Natural Resources Conservation Service, 2009; U.S. Geological Survey, 2008), which describes the geometry and attributes of the hydrologic units. Monthly streamflow data at included streamgages, adjusted for upstream water use, along with monthly climatic data and land-cover or basin characteristics provide a basis for developing regression equations to estimate natural monthly water yields at individual 
catchments. Streamflows are estimated from the catchment yields and flow is conserved through the NHDPlus network. Chloropleth maps of monthly water yield and flow can be generated and analyzed. Estimated monthly flows can be displayed within AFINCH, examined for nonstationarity, and tested for monotonic trends. Monthly flows also can be used to estimate flow-duration characteristics at stream segments within AFINCH.

\section{Purpose and Scope}

The purpose of this report is to document regression models and estimates of monthly water yields and flows developed for seven study areas spanning the U.S. Great Lakes Basin for the period 1951-2012. These models were developed within the AFINCH modeling environment by using the NHDPlus geospatial data framework to estimate monthly water yields and flows for 105,829 catchments and 107,691 flowlines in the region. The estimated flows are constrained to be consistent with measured flows at 823 U.S. Geological Survey (USGS) streamgages. These constraints also adjust regression estimates upstream from streamgages to generally improve flow estimates from regression estimates. Monthly water-use data were applied, where available, to mitigate effects of water use on the regionalization process.

\section{Description of the Great Lakes Basin}

The Great Lakes Basin in the northeastern United States and southeastern Canada contains the five Laurentian Great Lakes of Superior, Michigan, Huron, Erie, and Ontario. These Great Lakes make up the largest surface freshwater system on Earth (U.S. Environmental Protection Agency, 2014), and contain approximately 5,500 cubic miles $\left(\mathrm{mi}^{3}\right)$ of water (Waples and others, 2008). The approximate land drainage area of the basin is 201,460 square miles $\left(\mathrm{mi}^{2}\right)$, water area is $94,250 \mathrm{mi}^{2}$, and the total area of the basin is $296,000 \mathrm{mi}^{2}$ (Government of Canada and U.S. Environmental Protection Agency, 1995). Parts of the States of Michigan, New York, Ohio, Wisconsin, Indiana, Minnesota, Illinois, and Pennsylvania, and the provinces of Ontario and Quebec drain into the Great Lakes.

Hydrologic Region 04 subdivides the U.S. Great Lakes Basin into 15 hydrologic subregions based on surfacewater divides (watersheds) (U.S. Geological Survey, 2008) (fig. 1). The hierarchical hydrologic unit code (HUC) system describes increasingly refined levels of subdivision by appending a 2-digit code onto the end of the code identifying the previous level. In particular, the coding system divides surface-water drainage basins in the U.S. and the Caribbean into 21 hydrologic regions (2 digits), 221 subregions (4 digits), 378 accounting units ( 6 digits), and 2,264 cataloguing units ( 8 digits). The subregions and cataloguing units in the U.S. Great Lakes Basin were formed into seven study areas for analysis with AFINCH. These study areas facilitated the analysis by (1) combining hydrologically similar areas, (2) utilizing a larger pool of streamgaging stations than would have been possible by analyzing subregions individually, and (3) improving consistency of estimates between study areas.

\section{Data Integration within AFINCH and NHDPlus}

The AFINCH modeling approach and method of estimating flows along with descriptions of potential explanatory variables are described in the following sections.

\section{AFINCH Modeling Approach}

AFINCH implements a two-step predictor-corrector process. The predictor step is based on a user-specified multiple-linear-regression equation, which estimates monthly yields using selected monthly climatic and basin characteristics data and flows at streamgages. The corrector step adjusts the regression estimates to match measured flows at included streamgages.

\section{Predictor Step}

The predictor step in an AFINCH analysis is based on the statistical relation between a response variable that is a function of monthly flows and one or more explanatory variables that may be formed from time-varying monthly climatic data or fixed basin characteristics data. In particular, a user-specified multiple-linear-regression model is used to predict the response variable for each NHDPlus catchment in the study area. As discussed later in more detail, catchment yield (the response variable in this study) is defined as the streamflow contribution from the catchment divided by the catchment area. The estimated square root of yield is transformed and multiplied by catchment area to produce a runoff or catchment flow. The catchment flows are accumulated downstream by using the stream network topology described in NHDPlus. The following paragraphs develop the notational conventions underlying the AFINCH model. 


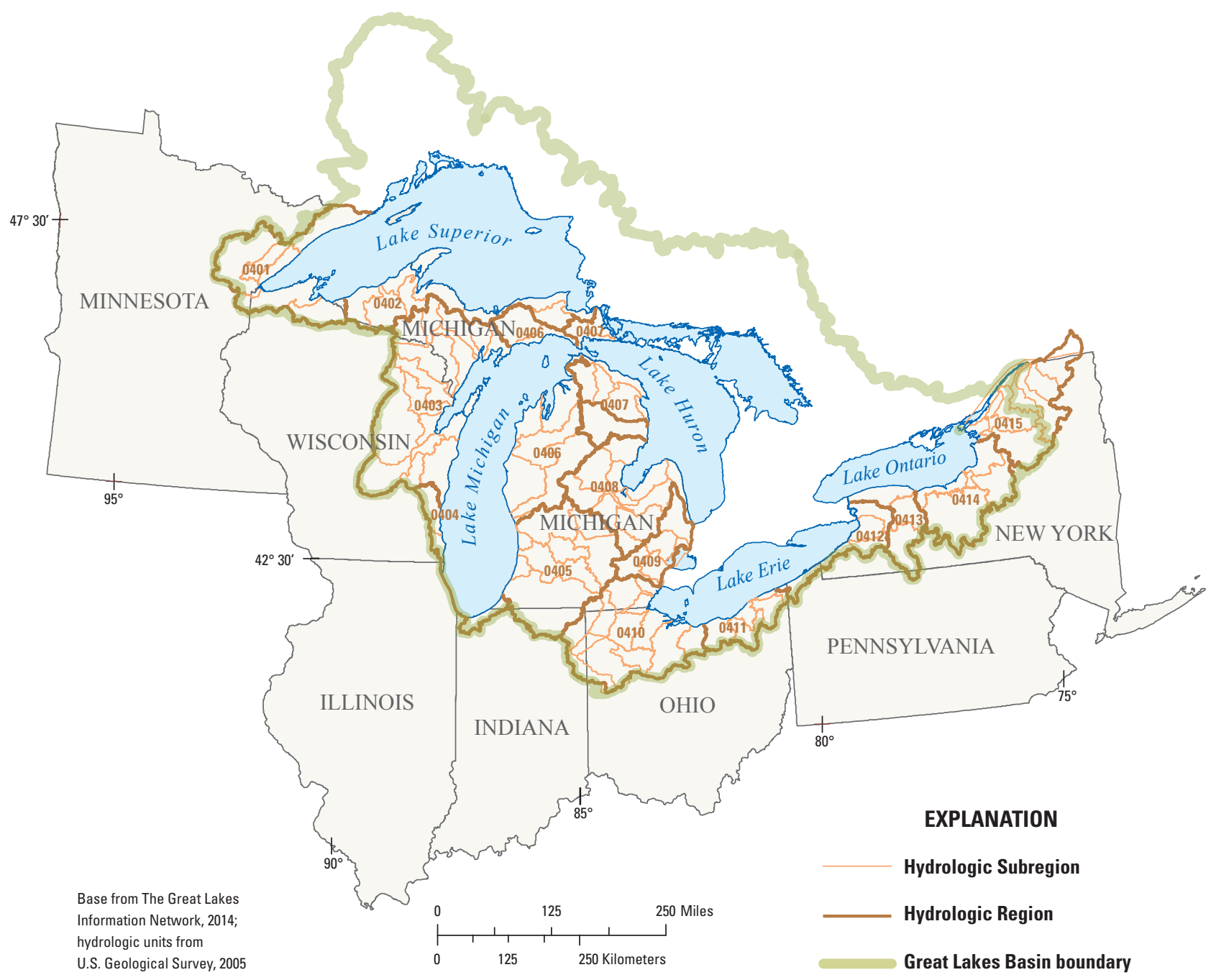

Figure 1. Hydrologic subregions and cataloguing units in the U.S. Great Lakes Basin.

A time series of monthly flows for the period of analysis at each NHDPlus flowline in the study area is the primary output from an AFINCH analysis. Monthly measured flows ${ }^{m e a} Q_{\text {Gage }(i, j)}$, for water year index $i$ and streamgage index $j$, are critical to developing these estimates. Streamflow data are typically reported as mean daily flows at the streamgage; monthly flows used in this study are the mean of the daily flows for each month. This set of monthly measured flows at included streamgages in water year $i$ can be formed into a column vector with length $n_{i}$, written ${ }^{\text {mea }} Q_{n_{i} \times 1}$.

In some stream reaches, withdrawals, diversions, or augmentations associated with water use can augment or diminish flows measured at downstream streamgages. If monthly water-use data are available to document these uses, measured flows at streamgages can be adjusted to more accurately represent natural flow conditions, that is, without water use. For example, if a consumptive water use of 1 cubic foot per second $\left(\mathrm{ft}^{3} / \mathrm{s}\right)$ is withdrawn from a reach upstream of one or more streamgages, the natural flow at the affected streamgages would be expected to have $1 \mathrm{ft}^{3} / \mathrm{s}$ more than the measured flow. Understanding natural flow conditions helps regionalize flow information with AFINCH. Measured flows that have been adjusted with available water use are designated as ${ }^{\text {mea }} \tilde{Q}_{\text {Gage }(i, j)}$. 
Streamflow generally occurs in dendritic drainage patterns in which headwater streams converge to form tributaries that combine flow from upstream branches. This process progresses downstream as tributaries continue to merge until only a main stem remains. A streamgaging network follows this pattern, so some streamflow is commonly measured by multiple streamgages. To avoid this redundancy in streamflow information, AFINCH computes an incremental flow, ${ }_{\Delta}^{\text {mea }} Q_{\text {Gage }(i, j)}$, and a corresponding incremental drainage area, ${ }_{\Delta}^{b a \sin } A_{\text {Gage }(i, j)}$, for each streamgage based on the stream network configuration. Consider, for example, a simple two-gage network in which the water measured at the upstream gage is combined with additional flow as it moves downstream and is re-measured at the downstream gage. In this network, the incremental flow and area for the upstream streamgage is the same as the measured flow and total area; at the downstream streamgage, however, the incremental flow is the measured flow minus the flow measured at the upstream gage. Similarly, the incremental area is the contributing drainage area at the downstream gage minus the contributing drainage area at the upstream gage.

Over a significant range of flows and drainage areas, (incremental) streamflow is proportional to the (incremental) drainage area. For example, all other factors being equal in a local flow system, the mean streamflow at a stream site with twice the drainage area of another stream site is likely to have twice the mean flow. The dominant effect of drainage areas on flows can mask more subtle effects statistically associated with the impact that land use and land cover, monthly precipitation and temperature, and other factors might have on streamflow. To increase the sensitivity of the analysis to these more subtle basin and climate effects, AFINCH analysis is based on water yield, which is obtained by dividing incremental flows by corresponding incremental areas as ${ }_{\Delta}^{m e a} Y_{\text {Gage }(i, j)}={ }_{\Delta}^{\text {mea }} Q_{\text {Gage }(i, j)} /{ }_{\Delta}^{\text {basin }} A_{\text {Gage }(i, j)}$ or in vector notation ${ }_{\Delta} Y_{n_{i} \times 1}={ }_{\Delta} Q_{n_{i} \times 1} \circ{ }_{\Delta}^{\text {basin }} A_{m_{i \times 1}}^{-1}$. This approach removes drainage area as a possible explanatory variable, which also mitigates some concerns about developing parameter estimates from analysis of (incremental) streamgage basins, made up of hundreds of individual NHDPlus catchments, for prediction at individual catchments.

A multiple regression model provides a basis for prediction and for assessing the uncertainty of the prediction. This uncertainty is based on simplifying assumptions concerning the characteristics and distribution of model residuals $\varepsilon$ formed by the differences between measured and predicted values of the response. In particular, model residuals are assumed to be independently and identically distributed with a normal (Gaussian) distribution having a mean of 0 and a variance of $\sigma_{\varepsilon}^{2}$ (Draper and Smith, 1998). So that this assumption is more likely satisfied, a square-root transformation was applied to the incremental yield to reduce its skewness. Skewness is a measure of the departure of a distribution from a symmetric distribution like the normal distribution. The transformed yield data are denoted by ${ }_{\Delta}^{\text {mea } Y_{\text {Gage }(i, j)}^{0.5}}=\sqrt{{ }_{\Delta}^{\text {mea }} Y_{\text {Gage }(i, j)}}$ and the residuals are formed as $\varepsilon_{n_{i} \times 1}={ }_{\Delta}^{m e a} Y_{n_{i} \times 1}^{0.5}-{ }_{\Delta}^{e s t} Y_{n_{i} \times 1}^{0.5}$.

In the multiple regression model underlying AFINCH, the response variable of monthly water yields - expressed generically for any individual water year $i$ with $n_{i}$ streamgages in the study area as ${ }_{\Delta}^{\text {mea }} Y_{n_{i}}^{0.5}$-is linearly related to a design matrix containing a column of 1's, corresponding to an intercept term, augmented on the right with a set of explanatory variables. Explanatory variables are user specified, but generally include monthly climatic and basin characteristics. All columns have the same number of elements (lengths of $n_{i}$ ) and the rows must be independent as 


$$
{ }_{\Delta}^{m e a} \tilde{Y}_{n_{i} \times 1}^{0.5}=\left[\begin{array}{ll}
\mathbf{1}_{n_{i} \times 1} & X_{n_{1} \times k}
\end{array}\right] \cdot \beta_{k+1}+\varepsilon_{n_{i}}
$$

where

$$
\begin{aligned}
& { }_{\Delta}^{m e a} \tilde{Y}_{n_{i} \times 1}^{0.5} \quad \text { is an } n_{i} \text {-column vector of the square-roots of measured incremental } \\
& \text { water yields, adjusted for water use; } \\
& {\left[\begin{array}{ll}
\mathbf{1}_{n_{i} \times 1} & X_{n_{i} \times k}
\end{array}\right] \quad \text { is a design matrix of explanatory variables in which the first }} \\
& \text { column is a } n_{i} \text {-vector of ones for the intercept term, } \\
& \text { which is augmented on the right by an } n_{i} \times k \text { matrix of } \\
& \text { explanatory variables indexed over water years by } i \text {; } \\
& \beta_{k+1} \quad \text { is a } k \times 1 \text { column vector of parameters relating the explanatory } \\
& \text { variables to the response variable; and } \\
& \varepsilon_{n_{i}} \quad \text { is an } n_{i} \text {-column vector of regression residuals, which are assumed } \\
& \text { to be independent and identically distributed random variables } \\
& \text { following a normal (Gaussian) distribution with mean } 0 \text { and variance } \sigma_{\varepsilon}^{2} \text {. }
\end{aligned}
$$

In cases where the $k$ explanatory variables can be partitioned into a set of $k_{v}$ time-varying characteristics such as monthly precipitation, and a set of $k_{f}$ time-invariant (fixed) characteristics such as soil types, the design matrix for a selected month in the $i^{\text {th }}$ year can be written as

$$
X_{n_{i} \times\left(1+k_{v}+k_{f}\right)}^{i}=\left[\begin{array}{lll}
1_{n_{i}} & X_{n_{i} \times k_{v}}^{i} & X_{n_{i} \times k_{f}}
\end{array}\right]
$$

Ordinary least-square (OLS) estimates of the parameter vector $\beta^{i}$ for monthly water yields in the $i^{\text {th }}$ water year can be computed (Draper and Smith, 1998) as

$$
\left[\begin{array}{c}
\hat{\beta}_{0}^{i} \\
\hat{\beta}_{k_{v}}^{i} \\
\hat{\beta}_{k_{f}}^{i}
\end{array}\right]=\left[\left(X_{n_{i} \times\left(1+k_{v}+k_{f}\right)}^{i}\right)^{\prime} \cdot X_{n_{i} \times\left(1+k_{v}+k_{f}\right)}^{i}\right]^{-1} \cdot\left(X_{n_{i} \times\left(1+k_{v}+k_{f}\right)}^{i}\right)^{\prime} \cdot{ }_{\Delta}^{m e a} \tilde{Y}_{n_{i} \times 1}^{0.5}
$$

Annual estimates of parameters may be useful in cases where the relation between the explanatory and response variables is suspected of being time varying. For example, a trend in parameter estimates might occur if an explanatory variable, such as land use, is treated as fixed when it is actually time varying. More commonly, the design matrix for the user-specified period of analysis (POA) for $N$ years can be developed by vertically stacking design matrixes for individual water years and corresponding water yield vectors ${ }_{\Delta}^{m e a} \tilde{Y}_{n_{i} \times 1}^{0.5}$ as

$$
\underline{X}=X_{\sum_{i} n_{i}, 1+k_{v}+k_{f}}=\left[\begin{array}{c}
X_{n_{1} \times\left(1+k_{v}+k_{f}\right)}^{1} \\
X_{n_{2} \times\left(1+k_{v}+k_{f}\right)}^{2} \\
\vdots \\
X_{n_{N} \times\left(1+k_{v}+k_{f}\right)}^{N}
\end{array}\right]=\left[\begin{array}{ccc}
1_{n_{1}} & X_{n_{i} \times k_{v}}^{i} & X_{n_{i} \times k_{f}} \\
1_{n_{2}} & X_{n_{2} \times k_{v}}^{2} & X_{n_{i} \times k_{f}} \\
\vdots & \vdots & \vdots \\
1_{n_{N}} & X_{n_{N} \times k_{v}}^{N} & X_{n_{i} \times k_{f}}
\end{array}\right]
$$


The corresponding OLS estimate of the regression parameter vector for the period of analysis is

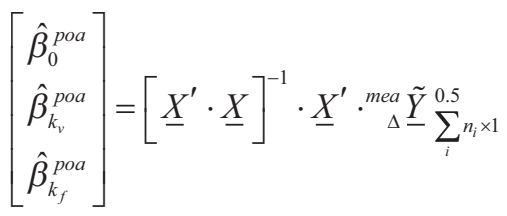

In AFINCH, 12 equations are individually estimated for monthly yields (either annually or for the POA) providing the flexibility to include different explanatory variables, $\beta$, in different monthly equations. A stepwise-selection process is used to automate the selection process in which the user specifies an $\alpha$-level (the probability of a type I error, which in this example, is incorrectly rejecting the null hypothesis that individual parameters are equal to zero) to control the apparent statistical significance of included explanatory variables. Stepwise selection is an iterative process in which $p$-values (the apparent probability that the magnitude of a parameter estimate, relative to its uncertainty, would occur by chance under the null hypothesis) are repeatedly computed and compared to the specified $\alpha$-level. Explanatory variables with $p$-values smaller than the $\alpha$-level are maintained in the equation. The user can override the stepwise-selection process by specifying which explanatory variables to include in the monthly regression equations regardless of their apparent statistical significance. Finally, the user can specify whether OLS or robust parameter estimates are to be used in the estimation of catchment water yields. Robust parameter estimates use a bisquare weight function with a tuning constant of 4.685 by default (The MathWorks, Inc., 2012). Robust estimates may be preferred to OLS estimates to reduce the sensitivity of parameter estimates to one or more irresolvable outliers in the water yield series; however, for the estimates presented in this report, OLS parameter estimates were used.

Once the monthly regression equations are developed from streamflow information at gaged basins, the results are applied to estimate water yields for all gaged and ungaged NHDPlus catchments. For the case where estimation equations are developed by using POA parameter estimates, monthly estimates of water yield for individual water years are computed as

$$
{ }^{e s t} \underline{\underline{y}}_{M \times 1}^{0.5}=\underline{x}_{M \times(k+1)} \cdot \hat{\beta}_{k+1}^{p o a},
$$

where

$$
\begin{aligned}
& { }^{e s t} \underline{\tilde{y}}_{M \times 1}^{0.5} \quad \text { is the estimated vector of natural water yields } \\
& \text { (adjusted for water use), in the square-root } \\
& \text { metric, for all MNHDPlus catchments-water } \\
& \text { years in the period of analysis; } \\
& \underline{x}_{M \times(k+1)} \quad \text { are catchment characteristics that correspond } \\
& \text { to basin characteristics for all the water-years } \\
& \text { in equation [4]; and } \\
& \hat{\beta}_{k+1}^{\text {poa }} \text { is the vector of parameter estimates. }
\end{aligned}
$$


Estimated runoff (flows) from individual catchments are computed from estimated yields and catchment areas as

$$
{ }^{e s t} \underline{\tilde{q}}_{M \times 1}=\left({ }^{e s t} \underline{\hat{y}}_{M \times 1}^{0.5}\right)^{2} \circ{ }^{\text {catch }} \underline{a}_{M \times 1},
$$

where

$$
\begin{aligned}
& { }^{e s t} \underline{\tilde{q}}_{M \times 1} \quad \text { is the vector of natural flows (runoff) for all } \\
& \text { catchments-water years in the period of } \\
& \text { analysis, } \\
& \left({ }^{e s t} \underline{\tilde{y}}_{M \times 1}^{0.5}\right)^{2} \quad \text { is the estimated natural catchment yield, and } \\
& { }^{\text {catch }} \underline{a}_{M \times 1} \quad \text { is a vector of NHDPlus catchment areas. }
\end{aligned}
$$

For notational simplicity, let ${ }^{e s t} \underline{\underline{q}}_{M \times 1} \rightarrow{ }^{e s t} \tilde{q}_{m \times 1}^{i} \forall w y(i) \in p o a$. Then accumulate monthly flows from catchments (runoff) forming the incremental areas upstream of included streamgages in water year $i$ as

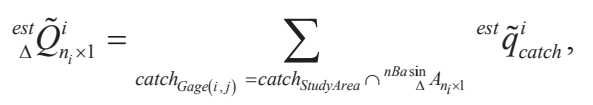

where

$$
\begin{aligned}
& { }_{\Delta}^{e s t} \tilde{Q}_{n_{i} \times 1}^{i} \quad \text { is the vector of accumulated AFINCH estimates of incremental } \\
& \text { flows at the } n_{i} \text { active streamgages in water year } i, \\
& \text { catch is the set of all study area catchments in the incremental area of } \\
& \text { individual streamgages monitored in water year } i \text {, and } \\
& { }^{e s t} \tilde{q}_{\text {catch }}^{i} \quad \text { is the natural monthly runoff from the set of catchments } \\
& \text { forming the incremental streamgage areas in the } i^{\text {th }} \text { water year. }
\end{aligned}
$$

and compare with measured flows. 


\section{Corrector Step}

A corrector step is used to adjust AFINCH estimated monthly water yields upstream from an included gage so that resulting AFINCH constrained monthly flows match measured flows at every streamgage for each water year in the period of analysis as ${ }_{\Delta}^{\text {con }} \tilde{Q}_{\text {Gage }_{j}}^{i} \equiv{ }_{\Delta}^{\text {mea }} \tilde{Q}_{\text {Gage }_{j}}^{i}$. The adjustment is applied to the catchment corresponding to the NHDPlus flowline where the streamgage is located and to estimated yields on all catchments upstream of the streamgage. The flow-based correction ratio is computed as

$$
\begin{aligned}
& \tilde{q} \operatorname{Ratio}_{\text {Gage }_{j}}^{i}={ }_{\Delta}^{\text {mea }} \tilde{Q}_{\text {Gage }_{j} \times 1} \circ{ }_{\Delta}{ }_{\Delta}^{e s t} \tilde{Q}_{\text {Gage }_{j} \times 1}^{-1} \\
& { }^{c o n} \tilde{y}_{\text {usGage }_{j}}^{i}={ }^{e s t} \tilde{y}_{\text {usGage }_{j}}^{i} \cdot \tilde{q} \text { Ratio }_{\text {Gage }_{j}}^{i},
\end{aligned}
$$

where

$$
\begin{aligned}
& { }^{c o n} \tilde{y}_{\text {usGage }}^{i} \quad \text { is the constrained estimate of water yields in NHDPlus } \\
& \text { catchments forming the incremental drainage area } \\
& \text { upstream of the } j^{\text {th }} \text { streamgage in the } i^{\text {th }} \text { water year, } \\
& \text { usGage }_{j} \quad \text { is the set of catchments contributing to flowlines } \\
& \text { upstream from } \text { Gage }_{j} \text {, and } \\
& \tilde{q} \text { Ratio }_{\text {Gage }_{j}}^{i} \quad \text { is a constant for the } j^{\text {th }} \text { streamgage and } i^{\text {th }} \text { water year. } \\
& \text { In ungaged areas and areas downstream from active } \\
& \text { gages, estimated water yields are unconstrained. }
\end{aligned}
$$

Constrained monthly water yields for each catchment and water year are used with corresponding catchment areas to compute constrained runoff ${ }^{c o n} \tilde{q}_{\text {catch }}^{i}={ }^{c o n} \tilde{y}_{\text {catch }}^{i} \circ a_{\text {catch }}$. The constrained runoffs are accumulated downstream by using the NHDPlus flowline topology to compute constrained accumulated AFINCH flows ${ }^{c o n} \tilde{Q}_{\text {flowline }}^{i}$. Lastly, wateruse information is added to affected flowlines and combined with ${ }^{c o n} \tilde{Q}_{\text {flowline }}^{i}$ so that ${ }^{\text {con }} Q_{\text {Gage }_{j}}^{i} \equiv{ }^{\text {mea }} Q_{\text {Gage }_{j}}^{i} \forall j \in$ Steamgage Network in water year $i$. 


\section{Model Limitations}

The regression model structure underlying AFINCH has some inconsistencies with the data structure that may affect the reliability of reported statistics ( $p$-values) used for assessing model suitability. In particular, model residuals are assumed to be independent in regression models, but the data structure provides repeated (annual) measures from a network of streamgages. Thus, residual series from individual streamgages may not be independent in time, and residual series from different streamgages may not be identically distributed. These limitations are likely to be exacerbated when there are few streamgages with relatively long periods of record. Lack of temporal independence and non-identically distributed residuals series could inflate the apparent significance (reducing $p$-values) of individual explanatory variables, thus leading to greater type I errors than what would be consistent with the specified $\alpha$ - level. To mitigate this potential problem, the number of explanatory variables potentially included in the regression model might be constrained, say to a number less than the square root of the number of included streamgages, or the $\alpha$-level might be otherwise reduced.

\section{NHDPlus Geospatial Data Frame}

NHDPlus describes the geometry and the attributes of the hydrologic units and links flowlines to catchments and to other flowlines. Notably, NHDPlus includes the flowline topology, which is the routing information providing the upstream and downstream connections for each flowline. AFINCH estimates of water yields are based on incremental flows and areas within streamgage networks. These incremental flows are summed down the network from the headwaters to the downstream-most segment in the watershed under analysis. There are 105,829 catchments making up Hydrologic Region 04 (U.S. Great Lakes Basin), which have an average area of $1.16 \mathrm{mi}^{2}$. Each catchment, which is uniquely identified by a Gridcode, is associated with a flowline (stream segment), which is uniquely identified by a ComID, that drains the catchment.

The 107,691 flowlines characterizing the drainage network in the U.S. Great Lakes Basin include streams and rivers, canals and ditches, pipelines, artificial paths, coastlines, and connectors. Flowlines in the U.S. Great Lakes Basin average about $1 \mathrm{mile}$ (mi) in length; some flowlines have no associated catchments. The topology of flowlines defined within NHDPlus provides a mechanism to accumulate flows from catchments and route flows through a drainage network. Initial inspection of some of the network design matrixes and the drainage area plots produced by AFINCH indicated the need for revisions to the flowline routing. In addition, early estimated flows also indicated the need for changes to the specified diversions for some flowlines. These revisions to flowline routing and diversion information are outlined in appendix 1 , table $1-1$.

\section{Response Data}

AFINCH uses multiple regression analysis to regionalize and spatially distribute water yields across catchments spanning (stream) gaged and ungaged basins in the study area. Monthly streamflow and water-use data are used together to describe the response variable.

Removing effects of artificial augmentations and diversions from directly impacted streamflow records helps describe natural streamflow conditions and associated yields, which can be regionalized. From these natural streamflow conditions, regression analysis describes how monthly climatic and basin characteristics affect the distribution of natural water yields across catchments. Catchment yields with corresponding drainage areas are used to compute runoff (flow), which can be accumulated downstream by using the NHDPlus streamflow topology. In gaged basins, the regression yield estimates are adjusted (constrained) so that resulting flows, with water-use data re-applied, match measured monthly flows. In ungaged basins, the natural water yields are used (without constraints) to estimate flows, with available water-use data re-applied to provide the final estimates of flow.

\section{Monthly Streamflow Data}

The NHDPlus GageLoc is a shapefile that locates USGS streamgages along flowlines. A USGS streamgage is uniquely identified by its station number; other information included in the analysis is the drainage area at the gage. In the U.S. Great Lakes Basin (Hydrologic Region 04), 1,339 streamgages are identified; however, stations that (1) represented lake sites, (2) had no specified drainage area, and (or) (3) did not have complete data for at least 1 water year within the period 1951-2012 were excluded from the analysis. Therefore, data from 823 streamgages were compiled for the AFINCH analysis. Time series of monthly streamflow values were computed from data obtained through the USGS cooperative streamgaging program (Wahl and others, 1995). 
Occasionally, within NHDPlus, one or more streamgages may be located at different points along a single flowline. The streamgage that best represents flow conditions, for example the gage with the longer period of record, was selected for the analysis. In each subregion, flow at sites that were regulated or influenced by water withdrawals and diversions, as indicated by station descriptions or analysis of the water yield graphs, was not included in the analysis if water-use or routing data were not readily available to adjust the flow to more natural conditions. NHDPlus routing tables were used to route flow upstream to headwaters or downstream to discharge points. For each streamgaging station, all upstream flowlines (and associated catchments) were identified by using the NHDPlusV2 Flow Table Navigator Toolbar in ArcMap (Horizon Systems Corporation, 2014).

\section{Monthly Water-Use Data}

In AFINCH, water use (including withdrawals, augmentations, and diversions) can be associated with flowlines and accounted for along with catchment flows. Monthly flows can be adjusted for specified water uses by using a companion (currently (2014) unpublished) program to AFINCH, which aids with water-use accounting and tracks withdrawals or returns from their point of origin to the downstream-most segment in the analysis. The Water Use Data Tool is available upon request from the USGS Michigan Water Science Center. Water withdrawals are indicated by negative water-use values, and flow augmentations are indicated by positive water-use values. Monthly water-use data for each water year within the period of analysis were specified for those flowlines with readily available data; however, determination of monthly water use for the period of analysis at each possible location was beyond the scope of this study. In addition, much of these data are unavailable, incomplete, or are not in a format that is readily amenable for inclusion in this analysis. Collection of additional data would likely improve the estimates and permit inclusion of additional streamgage data in the analysis.

Water withdrawals, diversions, and augmentations were determined from available records for selected flowlines in hydrologic subregions 0402, 0403, and 0409 using data from the National Pollutant Discharge Elimination System (NPDES) permit program, USGS Annual Data Reports, and (or) other USGS studies. The NPDES permit program requires industrial, municipal, and other facilities to report point-source discharges to surface-water bodies. Data were retrieved using the Permit Compliance System (U.S. Environmental Protection Agency, 2001). Monthly discharges that could be linked to an adjacent flowline were included in the AFINCH analysis each year the discharge occurred for subregions 0403 and 0409. Other withdrawal and diversion data were available from USGS Annual Data Reports (U.S. Geological Survey Water-Data Reports for Michigan and the St. Lawrence River Basin, 1958-2012). For example, reservoir stage data are collected that can be related to a flow or change in storage in the reservoir or to a withdrawal and later release to a different flowline. Data from these sources were included in the AFINCH analysis for subregions 0402 and 0403. In addition, local data used in the Kalamazoo area study (Luukkonen and others, 2004), describing water diversions to Portage Creek for selected years during 1966-2002, were used to estimate wateruse data for hydrologic subregion 0405 for this time period.

In hydrologic subregion 0402, at streamgaging station 04034000 Bond Falls Reservoir near Paulding, Michigan, water is diverted to South Branch Ontonagon River through Bond Falls Canal (streamgaging station 04033500), and water is used for power production at Victoria Dam near Rockland. Gage height data provided by Upper Peninsula Power Company are converted to acre-feet by USGS (Neal Craig, U.S. Geological Survey, oral commun., 2011). Water diverted from Schweitzer Reservoir to this subregion as described below also was included in the analysis. Data for 1950-2010 were used in the AFINCH analysis for subregion 0402.

In hydrologic subregion 0403, at streamgaging station 04057811 Greenwood Reservoir near Greenwood, Mich., water can flow over a concrete spillway into the Middle Branch Escanaba River below streamgaging station 04057814 Greenwood Release near Greenwood, Mich., with some downstream diversions by local industries above streamgaging station 04058000 . An outlet structure also permits flow from the reservoir into the afterbay (conservation pool) where water can be diverted to Green Creek gaged at Greenwood Diversion (streamgaging station 04057813) or released to the Middle Branch Escanaba River below streamgaging station 04057814. Beginning in October 1979, some water also was released after use to the East Branch Escanaba River via Goose Lake Outlet. Since January 1973, Greenwood Reservoir diverts some impounded water to Schweitzer reservoir (streamgaging station 04058190 Schweitzer Reservoir near Palmer, Mich.) for use in iron ore processing. Some flow from this reservoir is diverted to the city of Ishpeming, Mich., for municipal supply and discharged to the Carp River Basin (in hydrologic subregion 0402). After processing, water is returned to Green Creek or, beginning in 1980, to Goose Lake Outlet. Data for 1963-2009 were used in the AFINCH analysis for hydrologic subregion 0403. 


\section{Explanatory Data}

Multiple regression analyses relate explanatory variables consisting of time varying (monthly) climatic characteristics, and time invariant (fixed) catchment characteristics to monthly varying water yields. In this study, Parameter-elevation Regressions on Independent Slopes Model (PRISM) monthly precipitation and temperature data were used, and catchment characteristics in the National Land Cover Data (NLCD) set were supplemented or replaced with user-specified catchment characteristics. The source and characteristics of explanatory variables used in this analysis are described in the following sections.

\section{PRISM Monthly Climatic Data}

For this report, average monthly air temperature and total precipitation data were computed for each year during the period 1950-2012 for all catchments in the U.S. portion of the Great Lakes Hydrologic Region (04) (fig. 1). The climatic data are based on 2.5 arc-minute (approximately 4 kilometers) grids of PRISM data by Daly and Taylor (1998a, b). Monthly minimum and maximum air temperature and precipitation datasets for 1895-2012 from the PRISM Climate Group (2013) were overlain with polygons representing catchment boundaries to estimate spatially averaged precipitation and temperature values. The average of the minimum and maximum temperature values was used to estimate the average monthly temperature.

\section{National Land Cover Data}

Land-cover information for the conterminous United States for 2006 is available through the NLCD from the MultiResolution Land Characteristics Consortium (Fry and others, 2011). The 16-class land-cover classification scheme includes more broadly defined land-cover classes including water, developed, barren, forest, shrub land, herbaceous, planted or cultivated, and wetlands (appendix 1, table 1-2). Percentages of these categories were determined for each catchment in Hydrologic Region 04.

\section{NHDPlus National Catchment Characteristics Data}

NHDPlus includes a stream network based on the medium resolution (1:100,000 scale) NHD, with connectivity within the stream network and to associated catchments. Stream order is a method of assigning a numeric order to links within a stream network and in NHDPlus is a modified version of stream order as defined by Strahler (1957). The Strahler stream order algorithm does not account for flow splits in the network. The NHDPlus algorithm for stream order does take flow splits into consideration. These value-added attributes (VAA) are computed from upstream to downstream (McKay and others, 2012).

The NHDPlus suite of data includes information from the NED (Gesch and others, 2009). For each NHDPlus catchment, average catchment elevations were determined using the zonal statistics ArcMap toolbox command (ESRI, 2012). In a similar way, average and minimum catchment slope were determined for each catchment using the ArcMap toolbox commands for slope and zonal statistics (ESRI, 2012).

\section{User-Supplied Catchment Characteristics Data}

The user may provide one or more sets of shapefiles containing catchment attributes that can be used as explanatory variables in the subsequent regression analysis. User-specified variables for this analysis included soil information from the Soil Survey Geography (SSURGO) database (U.S. Department of Agriculture-Natural Resources Conservation Service, 2011), surficial and bedrock geology from the USGS National Gap Analysis Program (GAP), and growing degree day data (computed using PRISM air temperature data).

\section{SSURGO Soil Data}

The SSURGO database contains information about soil components and properties as collected by the National Cooperative Soil Survey and is available for most areas in the United States. The hydrologic group is based on estimates of runoff potential and are assigned according to the rate of water infiltration when the soils are not protected by vegetation, are thoroughly wet, and receive precipitation from long-duration storms (appendix 1, table 1-3). If a soil is assigned to a dual hydrologic group (A/D, B/D, or C/D), the first letter is for drained areas and the second is for undrained areas. Only the soils that in their natural condition are in group D are assigned to dual classes.

\section{GAP Vegetation and Land-Use Pattern Data}

The USGS National GAP Land Cover Data Set includes detailed vegetation and land-use patterns for the continental United States to aid in State or regional assessments of natural resources and to provide for a regionally consistent database with uniformity across State boundaries (U.S. Geological Survey, 2011). GAP is a nationwide effort under the direction 
of the USGS and provides information on the distribution of native vegetation types, modified and introduced vegetation, developed areas, and agricultural areas of the United States. Surficial and bedrock geology variables used in the AFINCH analysis are shown in appendix 1, table 1-4.

\section{Growing Degree Day Data}

Growing degree days provide a measure of heat accumulation because plant and animal development is related to the accumulation of heat or temperature units above a threshold or base temperature below which little growth occurs. The average annual growing degree days for the continental United States were calculated for 1971-2000 from downloaded PRISM air temperature datasets (Mike Slattery, U.S. Geological Survey, written commun., 2013). The base temperature (10 degrees Celsius $\left({ }^{\circ} \mathrm{C}\right)$ ) was subtracted from the average of the maximum and minimum monthly air temperatures. Only values equal to or greater than $0{ }^{\circ} \mathrm{C}$ were used and the resulting grid was then multiplied by the number of days in the month; the total from the previous month was added in a cumulative method to arrive at an approximation of the average annual growing degree days.

\section{Output Data}

The AFINCH program writes comma-delimited ASCII files with constrained and unconstrained monthly yields and flows for all the stream segments in the part of the NHDPlus area being analyzed and for each month and water year of the time period of the analysis. These flows and yields are indexed by the ComID of the stream segment or the Gridcode of the catchment. Headers in these files help document the analyses by describing explanatory variables, estimated parameters, and other user specifications. A representative chloropleth map showing the distribution of water yields for August averaged over 1951-2012 is shown in figure 2. An example time-series plot of monthly flows for a streamgaging station in hydrologic subregion 0403 showing the square root of measured flow with the square root of estimated incremental flow is shown in figure 3. Estimated flows and yields have been incorporated into a mapper application by the USGS Center for Integrated Data Analytics, http://cida.usgs.gov/glri/afinch/, for ease of use, display, and retrieval. These long-term flow series can be used to investigate trends or to characterize monthly flow durations.

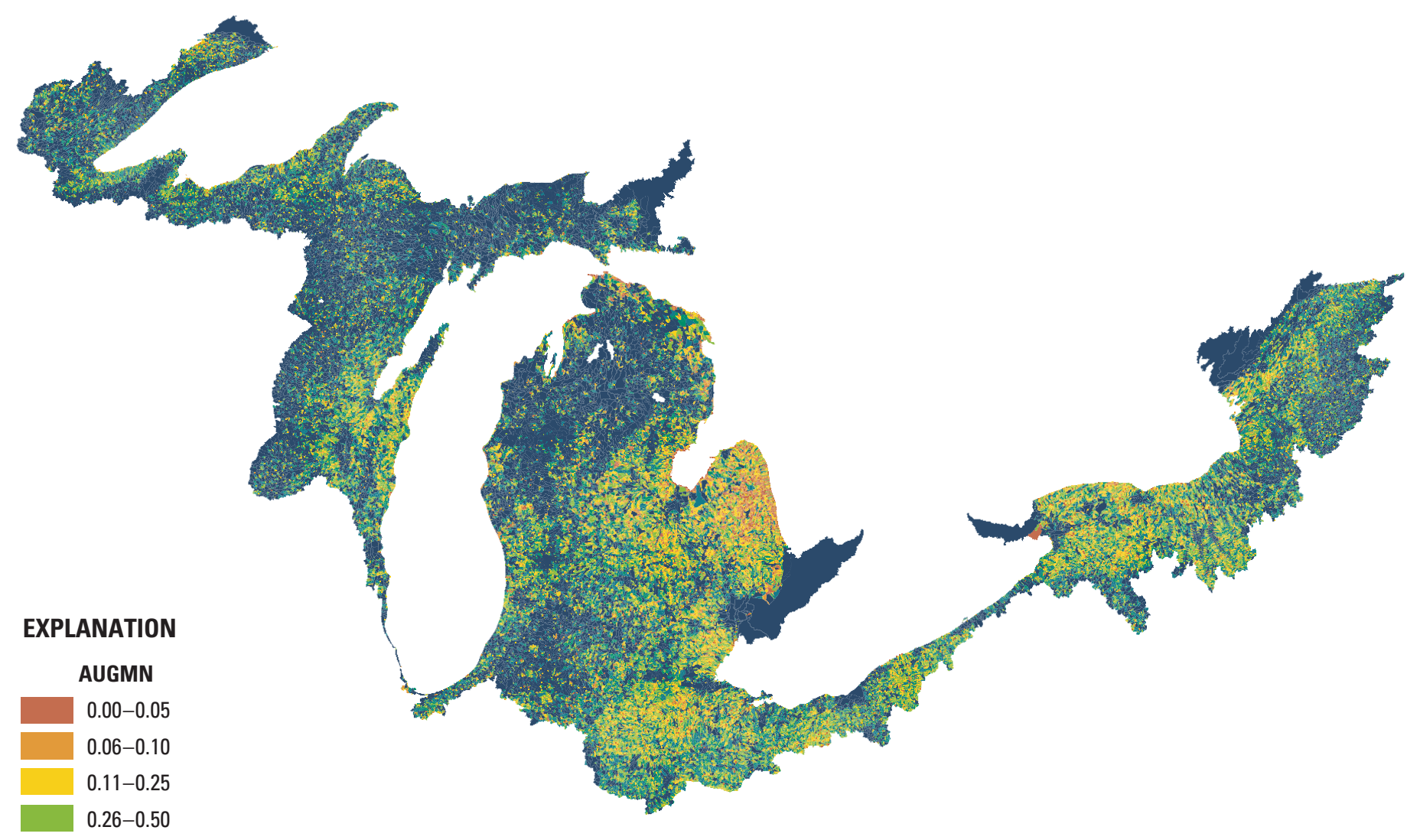

Figure 2. Distribution of estimated water yields for August averaged over 1951-2012. 


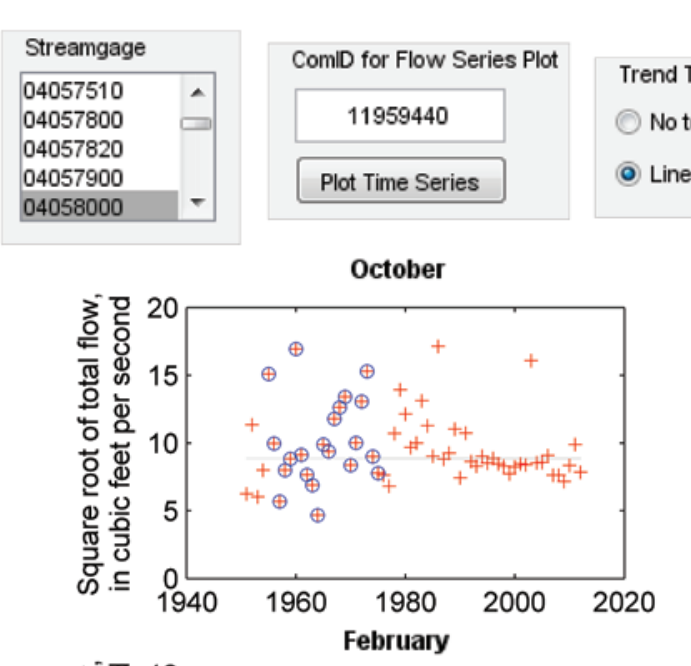

\begin{tabular}{|c|c|}
\hline rend Type & Trend Significance \\
\hline indicator & (-) $1 \% \bigcirc 10 \%$ \\
\hline Line & $5 \% \bigcirc 20 \%$ \\
\hline
\end{tabular}

\begin{tabular}{l} 
File Plotted Data \\
Output Path \\
C:HomelclluukkolEcoflowsiAFinchHR04LMB_westioutputtFlowSeriesi \\
\hline Output File \\
\hline C11959440Series.dat \\
\hline
\end{tabular}

Explanation

+ Estimated flows

- Measured flows

-. Median

- Trend
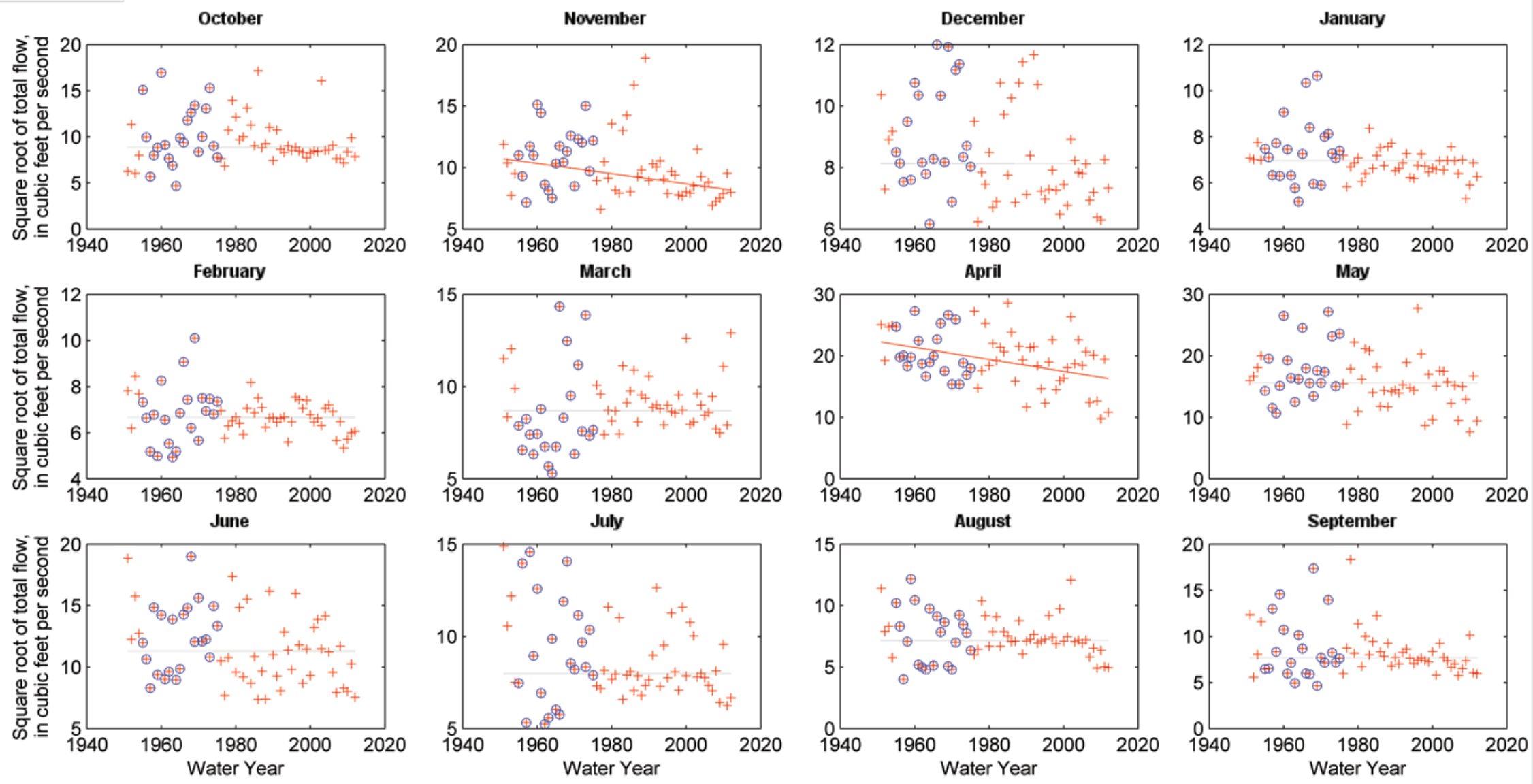

Figure 3. Time-series plot of monthly flows at flowline 11959440, streamgaging station 04058000, Middle Branch Escanaba River near Ishpeming, Michigan. 


\section{Methods of Flow and Water-Yield Estimation for Study Areas}

Flows and water yields for water years 1951-2012 were estimated for 107,691 flowlines and 105,829 catchments in Hydrologic Region 04 representing the portion of the Great Lakes Basin in the U.S. (fig. 1). The 15 hydrologic subregions were combined to form 7 study areas consisting of multiple subregions and (or) cataloguing units for analysis with AFINCH (table 1). Details on the analysis and estimation of flows and water yields are presented for each study area. Each section includes (1) location map, (2) table of the streamgaging stations in the study area indicating each year each streamgage was active during the analysis period and whether or not the streamgage data were included in the analysis, (3) the regression coefficients and fit statistics for each variable, and (4) graph showing the $t$-values for each explanatory variable by month.

The regression coefficients indicate the size and direction (positive or negative) of the effect that each variable has on the yield estimate. However, some of the differences in coefficient magnitudes are owing to the fact that the potential explanatory variables are in different units, for example, variables with larger values would likely have smaller coefficients. To aid in the interpretation of the effect of each variable, graphs of $t$-values for all variables in the multiple-linear-regression equation are shown for each study area. The magnitudes of the $t$-values are test statistics that are used to interpret the likelihood that individual parameters in the regression equation are equal to zero. The sign of the $t$-values indicate whether the corresponding parameter is positive or negative. The cells in the image are white if the magnitude of the corresponding test statistic did not indicate significance at the level prescribed by the user. Cells are graded with increasing tones of red for increasingly significant parameters with negative signs. Similarly, cells are graded with increasing tones of blue for increasingly significant parameters with positive signs. The color scale at the bottom indicates the magnitude of the $t$-statistics. This visual representation permits interpretation of the information used to estimate water yields by each variable and for each month. For example, parameters (represented in each horizontal band) with most months tinted in shades of blue and (or) red likely provide more information in estimating the water-yield response than would a variable where most months are white indicating that the test statistics were not significant. Likewise, months (represented in each vertical band) with most cells tinted blue and (or) red would be estimated using multiple parameters, whereas months where most cells are white would not be estimated using much parameter information. The regression-fit statistics include the rootmean-square error (RMSE), F statistic, and the coefficient of determination $\left(\mathrm{R}^{2}\right)$ for the fit to the square-root of water yield. As noted previously, drainage area is so strongly correlated to streamflow that this relation is built into the analysis by writing the regression equation in terms of yield (streamflow divided by drainage area). Examination of the tables reveals that the reported $\mathrm{R}^{2}$ values for the fit to the square root of yield can be fairly low (0.2-0.7); however, the corresponding $\mathrm{R}^{2}$ for the fit of estimated to measured streamflows can be quite high $(0.8-0.9)$ because of the strong correlation between area and streamflow.

Selection of explanatory variables was guided by knowledge of each study area, identification of variables likely to affect water yields, and inspection of the images showing monthly parameter $t$-values. Furthermore, an additional consideration was to improve the estimation of yields and flows during April (higher flow month) and August (lower flow month) in order for the estimates to be more useful for

Table 1. Summary of study area information.

\begin{tabular}{|c|c|c|c|c|c|}
\hline $\begin{array}{l}\text { Study } \\
\text { area }\end{array}$ & $\begin{array}{l}\text { Hydrologic subregions (4-digits) and (or) } \\
\text { cataloguing units (8-digits) }\end{array}$ & $\begin{array}{l}\text { Drainage area } \\
\text { (square miles) }\end{array}$ & $\begin{array}{l}\text { Number of } \\
\text { streamgages }\end{array}$ & $\begin{array}{l}\text { Number of } \\
\text { flowlines }\end{array}$ & $\begin{array}{l}\text { Number of } \\
\text { catchments }\end{array}$ \\
\hline 1 & 0401 and 0402 & 16,632 & 76 & 15,442 & 15,221 \\
\hline 3 & 0405 and 04040001 & 13,620 & 105 & 11,523 & 11,300 \\
\hline 4 & $\begin{array}{l}\text { 04060101, 04060102, 04060103, 04060104, } \\
\text { 04060105, 04070003, } 04070004,04070005 \\
04070006 \text {, and } 04070007\end{array}$ & 14,844 & 61 & 7,946 & 7,808 \\
\hline 5 & 0408 and 0409 & 12,881 & 116 & 13,303 & 13,151 \\
\hline 6 & 0410 and 0411 & 14,942 & 120 & 16,935 & 16,713 \\
\hline 7 & $0412,0413,0414$, and 0415 & 21,655 & 168 & 26,013 & 25,483 \\
\hline
\end{tabular}


ecological stream-classification work. The variables selected for each study area represent those with the most significance of the combinations that were considered during the regression analysis; however, consideration of all possible variables and combinations was beyond the scope of this report. It is possible that there are areas where local conditions differ greatly from the variable characteristics that were selected for the study area and that in these areas flow and yield estimates are not as representative of actual conditions than they could be with a different variable set. It also is possible that a regionally important variable was not included in the analysis. Equally likely is the potential that major water uses (withdrawals, augmentations, or diversions) were not accounted for during the analysis. However, regionally these estimates present a reasonable, consistent, and defensible set of flow and yield estimates for the U.S. Great Lakes Basin.

\section{Study Area 1}

Study area 1 is formed by hydrologic subregions 0401 and 0402 in the western part of the U.S. Great Lakes Basin and encompasses parts of Minnesota, Wisconsin, and the Upper Peninsula of Michigan (fig. 4). The number of stations included in the analysis ranged from 17 (1986, 1998, 1999, 2001, 2007, 2008, and 2009) to 30 (1954 and 1955) (table 2). Nine explanatory variables were selected during the regression analysis with the number of variables specified for each monthly equation ranging from 6 (June) to 8 (October, November, December, January, April, May, and August). Selected explanatory variables are summarized in table 3 . Variables and regression statistics based on the stepwise regression for each month are indicated in table 4 and figure 5.

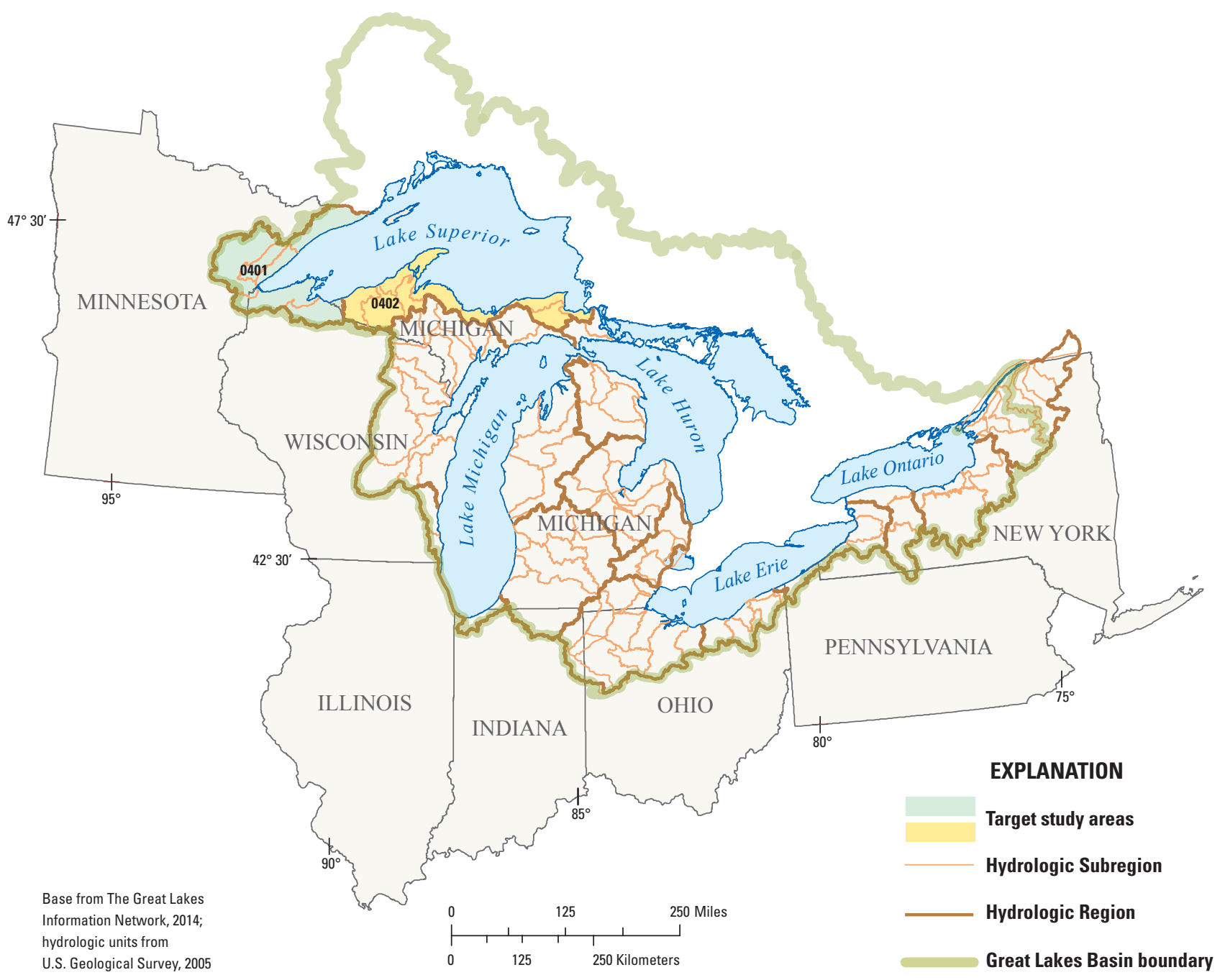

Figure 4. Location of study area 1 (hydrologic subregions 0401 and 0402) in the U.S. Great Lakes Basin. 
Table 2 Streamgaging stations considered for analysis of study area 1 (hydrologic subregions 0401 and 0402 ) in the U.S. Great Lakes Basin, by water year.

$\underline{[\bullet, \text { station active and included in the analysis; } \circ \text {, station active but not included in the analysis] }}$

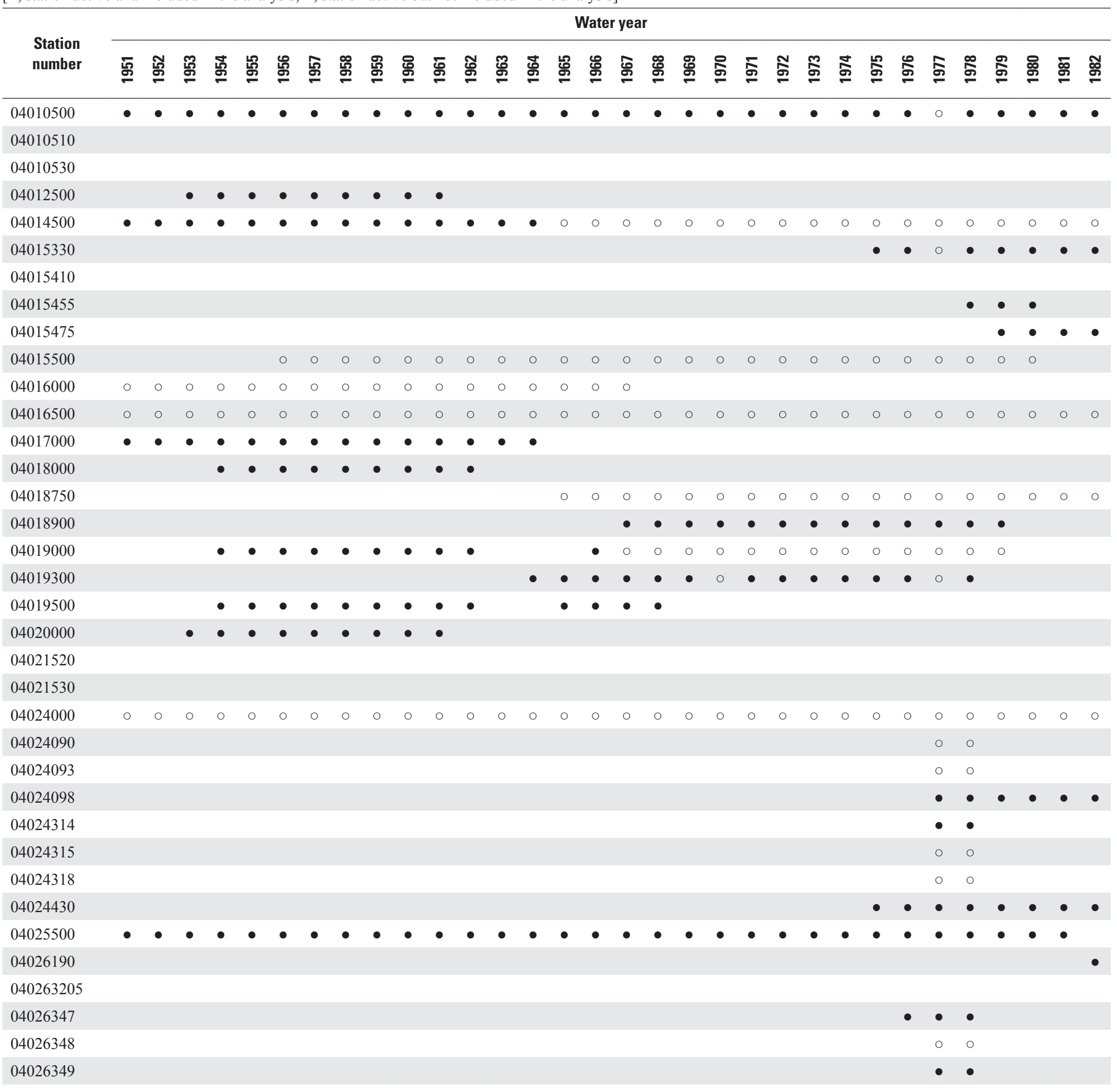

040263491 
Table 2. Streamgaging stations considered for analysis of study area 1 (hydrologic subregions 0401 and 0402) in the U.S. Great Lakes Basin, by water year.-Continued

$[\bullet$, station active and included in the analysis; $\odot$, station active but not included in the analysis]

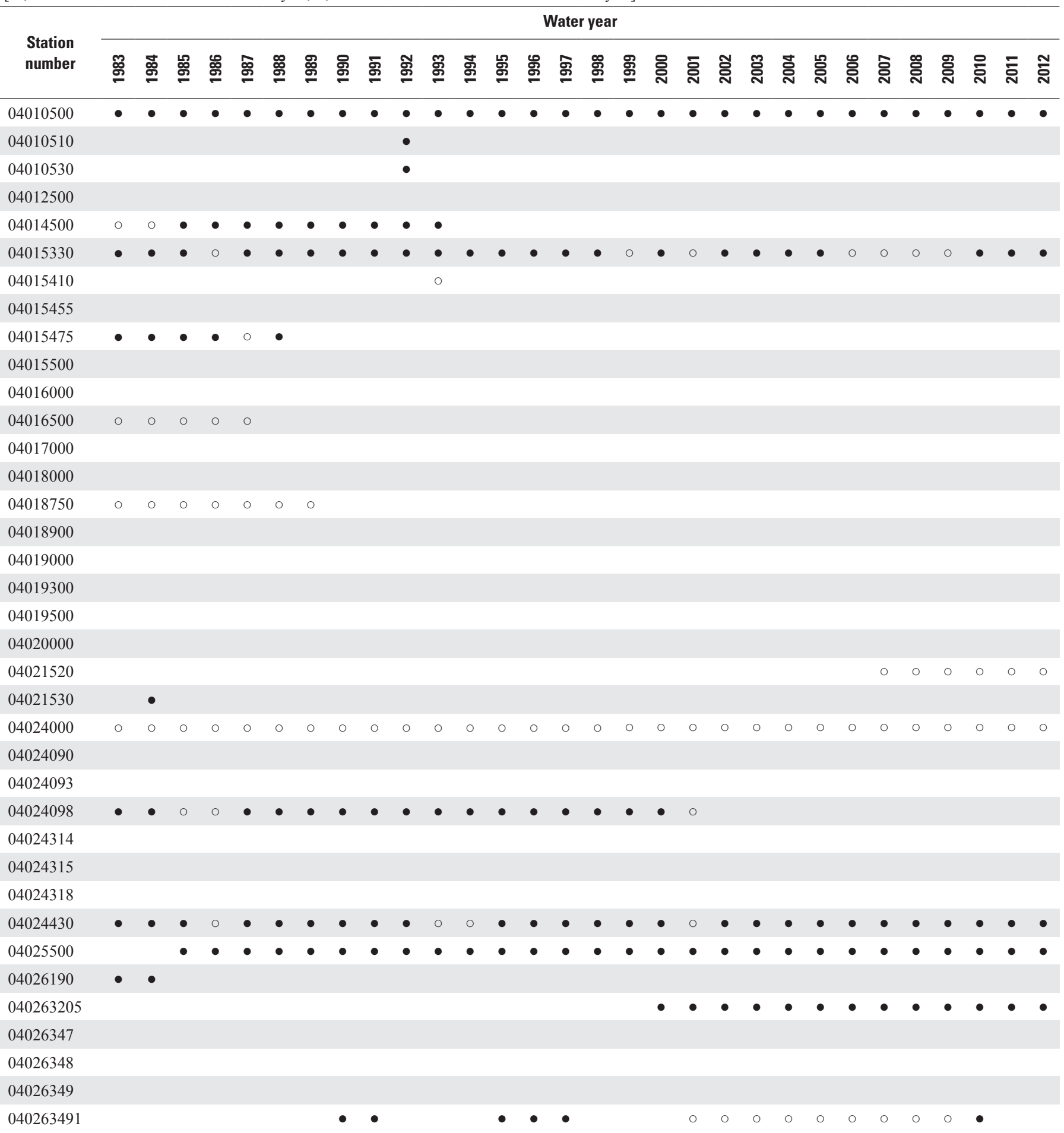


Table 2 Streamgaging stations considered for analysis of study area 1 (hydrologic subregions 0401 and 0402) in the U.S. Great Lakes Basin, by water year.-Continued

$[\bullet$, station active and included in the analysis; $\circ$, station active but not included in the analysis]

\section{Water year}

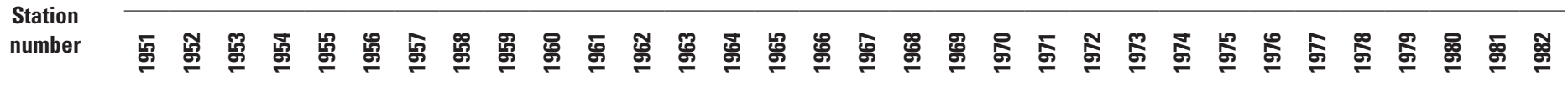

\begin{tabular}{|c|c|c|c|c|c|c|c|c|c|c|c|c|c|c|c|c|c|c|c|c|c|c|c|c|c|c|c|c|c|c|c|c|}
\hline 04026450 & & & & & & & & & & & & & & & & & & & & & - & 0 & • & - & - & & & & & & & \\
\hline 04026500 & 0 & 0 & $\bullet$ & $\bullet$ & $\bullet$ & & & & & & & & & & & & & & & & & & & & & & & & & & & \\
\hline 04026870 & & & & & & & & & & & & & & & & & & & & & & & $\circ$ & $\circ$ & o & o & o & & & & & \\
\hline 04027000 & $\bullet$ & $\bullet$ & $\bullet$ & $\bullet$ & $\bullet$ & $\bullet$ & $\bullet$ & $\bullet$ & $\bullet$ & $\bullet$ & $\bullet$ & $\bullet$ & $\bullet$ & $\bullet$ & $\bullet$ & $\bullet$ & $\bullet$ & $\bullet$ & $\bullet$ & $\bullet$ & $\bullet$ & • & $\circ$ & $\bullet$ & $\bullet$ & $\bullet$ & - & - & $\bullet$ & • & • & $\bullet$ \\
\hline 04027500 & $\bullet$ & $\bullet$ & $\bullet$ & - & $\bullet$ & $\bullet$ & $\bullet$ & - & $\bullet$ & $\bullet$ & $\bullet$ & $\bullet$ & $\bullet$ & $\bullet$ & $\bullet$ & $\bullet$ & $\bullet$ & $\bullet$ & $\bullet$ & $\bullet$ & $\bullet$ & $\bullet$ & $\bullet$ & $\bullet$ & $\bullet$ & $\bullet$ & $\bullet$ & $\bullet$ & $\bullet$ & $\bullet$ & $\bullet$ & $\bullet$ \\
\hline 04028000 & $\bullet$ & $\bullet$ & $\bullet$ & $\bullet$ & & & & & & & & & & & & & & & & & & & & & & & & & & & & \\
\hline \multicolumn{33}{|l|}{04029990} \\
\hline 04030000 & $\bullet$ & $\bullet$ & $\bullet$ & $\bullet$ & $\bullet$ & $\bullet$ & $\bullet$ & $\bullet$ & $\bullet$ & $\bullet$ & $\bullet$ & $\bullet$ & $\bullet$ & $\bullet$ & $\bullet$ & $\bullet$ & $\bullet$ & $\bullet$ & $\bullet$ & $\bullet$ & & & & & & & & & & & & \\
\hline 04031000 & & & & & $\bullet$ & $\bullet$ & • & $\bullet$ & $\bullet$ & $\bullet$ & $\bullet$ & $\bullet$ & $\bullet$ & $\bullet$ & $\bullet$ & $\bullet$ & • & $\bullet$ & • & $\bullet$ & • & $\bullet$ & $\bullet$ & $\bullet$ & $\bullet$ & $\bullet$ & - & $\bullet$ & $\bullet$ & $\bullet$ & • & • \\
\hline 04031500 & $\bullet$ & • & $\bullet$ & $\bullet$ & $\bullet$ & $\bullet$ & $\bullet$ & • & $\bullet$ & $\bullet$ & $\bullet$ & $\bullet$ & $\bullet$ & $\bullet$ & $\bullet$ & $\bullet$ & • & $\bullet$ & $\bullet$ & $\bullet$ & - & $\bullet$ & $\bullet$ & $\bullet$ & $\bullet$ & $\bullet$ & - & - & $\bullet$ & - & • & • \\
\hline 04032000 & $\bullet$ & - & - & - & $\bullet$ & • & $\bullet$ & $\bullet$ & $\bullet$ & $\bullet$ & $\bullet$ & $\bullet$ & $\bullet$ & $\bullet$ & $\bullet$ & • & $\bullet$ & $\bullet$ & $\bullet$ & • & - & $\bullet$ & $\bullet$ & & & & & & & & & \\
\hline 04032500 & & & $\bullet$ & • & $\bullet$ & $\bullet$ & • & & & & & & & & & & & & & & & & & & & & & & & & & \\
\hline 04033000 & $\bullet$ & • & - & $\bullet$ & $\bullet$ & $\bullet$ & $\bullet$ & $\bullet$ & $\bullet$ & $\bullet$ & $\bullet$ & $\bullet$ & $\bullet$ & $\bullet$ & $\bullet$ & • & $\bullet$ & $\bullet$ & $\bullet$ & $\bullet$ & - & $\bullet$ & $\bullet$ & $\bullet$ & $\bullet$ & $\bullet$ & - & $\bullet$ & $\bullet$ & • & $\bullet$ & $\bullet$ \\
\hline 04033500 & $\circ$ & $\circ$ & $\circ$ & $\circ$ & $\circ$ & $\circ$ & $\circ$ & $\circ$ & $\circ$ & $\circ$ & $\circ$ & $\circ$ & $\circ$ & $\circ$ & $\circ$ & $\circ$ & $\circ$ & $\circ$ & $\circ$ & $\circ$ & $\circ$ & $\circ$ & $\circ$ & $\circ$ & $\circ$ & $\circ$ & $\circ$ & $\circ$ & $\circ$ & ○ & ○ & $\circ$ \\
\hline 04034500 & $\bullet$ & - & $\bullet$ & - & $\bullet$ & - & $\bullet$ & • & $\bullet$ & $\bullet$ & $\bullet$ & • & • & $\bullet$ & $\bullet$ & • & $\bullet$ & $\bullet$ & $\bullet$ & $\bullet$ & - & $\bullet$ & $\bullet$ & $\bullet$ & $\bullet$ & • & - & - & $\bullet$ & - & • & • \\
\hline 04035000 & $\bullet$ & • & $\bullet$ & - & $\bullet$ & • & $\bullet$ & • & $\bullet$ & $\bullet$ & $\bullet$ & $\bullet$ & $\bullet$ & $\bullet$ & $\bullet$ & $\bullet$ & $\bullet$ & $\bullet$ & $\bullet$ & $\bullet$ & - & $\bullet$ & $\bullet$ & $\bullet$ & $\bullet$ & $\bullet$ & - & $\bullet$ & $\bullet$ & & & \\
\hline 04035500 & $\bullet$ & $\bullet$ & $\bullet$ & $\bullet$ & • & $\bullet$ & $\bullet$ & $\bullet$ & $\bullet$ & $\bullet$ & $\bullet$ & $\bullet$ & $\bullet$ & $\bullet$ & $\bullet$ & $\bullet$ & $\bullet$ & $\bullet$ & $\bullet$ & $\bullet$ & $\bullet$ & $\bullet$ & $\bullet$ & $\bullet$ & $\bullet$ & $\bullet$ & • & $\bullet$ & $\bullet$ & $\bullet$ & $\bullet$ & $\bullet$ \\
\hline 04036000 & $\bullet$ & $\bullet$ & $\bullet$ & $\bullet$ & $\bullet$ & $\bullet$ & $\bullet$ & $\bullet$ & $\bullet$ & $\bullet$ & $\bullet$ & $\bullet$ & $\bullet$ & $\bullet$ & $\bullet$ & $\bullet$ & $\bullet$ & $\bullet$ & $\bullet$ & $\bullet$ & - & $\bullet$ & $\bullet$ & $\bullet$ & $\bullet$ & $\bullet$ & $\bullet$ & $\bullet$ & $\bullet$ & - & $\bullet$ & $\bullet$ \\
\hline 04037500 & $\bullet$ & - & • & - & $\bullet$ & • & $\bullet$ & $\bullet$ & $\bullet$ & $\bullet$ & $\bullet$ & $\bullet$ & $\bullet$ & $\bullet$ & $\bullet$ & • & $\bullet$ & $\bullet$ & $\bullet$ & • & - & $\bullet$ & $\bullet$ & $\bullet$ & $\bullet$ & • & - & - & $\bullet$ & - & - & $\bullet$ \\
\hline 04039500 & $\bullet$ & $\bullet$ & • & • & $\bullet$ & • & $\bullet$ & • & $\bullet$ & $\bullet$ & $\bullet$ & $\bullet$ & $\bullet$ & $\bullet$ & $\bullet$ & $\bullet$ & $\bullet$ & $\bullet$ & $\bullet$ & $\bullet$ & $\bullet$ & & & & & & & & & & & \\
\hline 04040000 & $\bullet$ & $\bullet$ & - & $\bullet$ & $\bullet$ & $\bullet$ & $\bullet$ & $\bullet$ & $\bullet$ & $\bullet$ & $\circ$ & $\bullet$ & o & $\circ$ & $\bullet$ & $\bullet$ & $\circ$ & $\bullet$ & $\bullet$ & $\bullet$ & $\circ$ & $\bullet$ & $\bullet$ & $\bullet$ & $\bullet$ & $\bullet$ & - & $\bullet$ & $\bullet$ & • & $\bullet$ & $\bullet$ \\
\hline 04040500 & $\bullet$ & $\bullet$ & $\bullet$ & • & • & • & • & • & $\bullet$ & $\bullet$ & $\bullet$ & $\bullet$ & $\bullet$ & $\bullet$ & $\bullet$ & $\bullet$ & $\bullet$ & $\bullet$ & $\bullet$ & $\bullet$ & - & • & $\bullet$ & $\bullet$ & $\bullet$ & $\bullet$ & - & - & $\bullet$ & • & • & • \\
\hline 04041500 & $\bullet$ & $\bullet$ & $\bullet$ & $\bullet$ & $\bullet$ & • & $\bullet$ & • & $\bullet$ & $\bullet$ & $\bullet$ & $\bullet$ & $\bullet$ & $\bullet$ & $\bullet$ & $\bullet$ & $\bullet$ & $\bullet$ & $\bullet$ & $\bullet$ & $\bullet$ & $\bullet$ & $\bullet$ & $\bullet$ & $\bullet$ & $\bullet$ & $\bullet$ & $\bullet$ & $\bullet$ & $\bullet$ & $\bullet$ & $\bullet$ \\
\hline 04042500 & $\bullet$ & - & $\bullet$ & - & $\bullet$ & - & $\bullet$ & • & $\bullet$ & $\bullet$ & $\bullet$ & • & $\bullet$ & $\bullet$ & $\bullet$ & • & $\bullet$ & $\bullet$ & $\bullet$ & • & $\bullet$ & • & & & & & & & & & & \\
\hline 04043000 & $\bullet$ & • & - & $\bullet$ & $\bullet$ & $\bullet$ & • & $\bullet$ & $\bullet$ & $\bullet$ & $\bullet$ & $\bullet$ & $\bullet$ & $\bullet$ & $\bullet$ & $\bullet$ & o & $\bullet$ & $\bullet$ & $\bullet$ & $\bullet$ & $\bullet$ & $\bullet$ & $\bullet$ & & & & & & & & \\
\hline 04043050 & & & & & & & & & & & & & & & & & $\bullet$ & $\bullet$ & $\bullet$ & $\bullet$ & $\bullet$ & $\bullet$ & $\bullet$ & $\bullet$ & $\bullet$ & $\bullet$ & $\bullet$ & $\bullet$ & $\bullet$ & $\bullet$ & $\bullet$ & $\bullet$ \\
\hline \multicolumn{33}{|l|}{04043140} \\
\hline \multicolumn{33}{|l|}{04043150} \\
\hline \multicolumn{33}{|l|}{04043238} \\
\hline \multicolumn{33}{|l|}{04043244} \\
\hline \multicolumn{33}{|l|}{04043275} \\
\hline \multicolumn{33}{|l|}{04043800} \\
\hline 04044400 & & & & & & & & & & & & $\bullet$ & $\bullet$ & $\bullet$ & $\bullet$ & $\bullet$ & $\bullet$ & $\bullet$ & $\bullet$ & $\bullet$ & $\bullet$ & $\bullet$ & $\bullet$ & $\bullet$ & $\bullet$ & $\bullet$ & $\bullet$ & $\bullet$ & $\bullet$ & $\bullet$ & • & $\bullet$ \\
\hline 04044563 & & & & & & & & & & & & & & & & & & & & & & & & & & & & & & • & $\bullet$ & \\
\hline 04044573 & & & & & & & & & & & & & & & & & & & & & & & & & & & & & & • & - & \\
\hline 04044583 & & & & & & & & & & & & & & & & $\circ$ & $\circ$ & $\circ$ & $\circ$ & $\circ$ & & & & & & & & & & $\circ$ & ○ & \\
\hline 04044595 & & & & & & & & & & & & & & & & & & & & & & & & & & & & & & - & • & \\
\hline \multicolumn{33}{|l|}{04044724} \\
\hline 04045500 & & & & $\bullet$ & • & $\bullet$ & $\bullet$ & • & $\bullet$ & $\bullet$ & $\bullet$ & $\bullet$ & $\bullet$ & $\bullet$ & $\bullet$ & $\bullet$ & $\bullet$ & $\bullet$ & $\bullet$ & $\bullet$ & $\bullet$ & $\bullet$ & $\bullet$ & $\bullet$ & $\bullet$ & $\bullet$ & $\bullet$ & $\bullet$ & $\bullet$ & $\bullet$ & $\bullet$ & • \\
\hline
\end{tabular}


Table 2. Streamgaging stations considered for analysis of study area 1 (hydrologic subregions 0401 and 0402 ) in the U.S. Great Lakes Basin, by water year.-Continued

[ $\bullet$, station active and included in the analysis; $\odot$, station active but not included in the analysis]

\begin{tabular}{|c|c|c|c|c|c|c|c|c|c|c|c|c|c|c|c|c|c|c|c|c|c|c|c|c|c|c|c|c|c|c|c|}
\hline \multirow{2}{*}{$\begin{array}{l}\text { Station } \\
\text { number }\end{array}$} & \multicolumn{31}{|c|}{ Water year } \\
\hline & ஜ̋ & 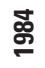 & 농 & $\stackrel{\circ}{\circ}$ & ळ & 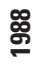 & \% & ஓ्. & চু & ซั & ஜூ & \% & 농 & $\stackrel{\circ}{\circ}$ & \% & \% & \% & ర్ & ర్రై & สิ & กั & ర్రి & 농 & స్ & ธ్రి & 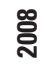 & 오․ & กั & હ & & กั \\
\hline \multicolumn{32}{|l|}{04026450} \\
\hline \multicolumn{32}{|l|}{04026500} \\
\hline \multicolumn{32}{|l|}{04026870} \\
\hline 04027000 & - & - & - & - & - & - & - & - & - & - & - & - & - & - & $\bullet$ & • & - & • & • & $\bullet$ & - & - & - & $\bullet$ & $\bullet$ & • & - & - & - & & $\bullet$ \\
\hline 04027500 & $\bullet$ & $\bullet$ & $\bullet$ & $\bullet$ & $\bullet$ & $\bullet$ & $\bullet$ & $\bullet$ & $\bullet$ & $\bullet$ & $\bullet$ & $\bullet$ & $\bullet$ & $\bullet$ & $\bullet$ & $\bullet$ & $\bullet$ & $\bullet$ & $\bullet$ & $\bullet$ & $\bullet$ & $\bullet$ & $\bullet$ & $\bullet$ & $\bullet$ & $\bullet$ & $\bullet$ & $\bullet$ & . & & $\bullet$ \\
\hline \multicolumn{32}{|l|}{04028000} \\
\hline 04029990 & & & & & $\circ$ & $\circ$ & $\circ$ & $\circ$ & $\circ$ & $\circ$ & $\bullet$ & $\bullet$ & o & $\circ$ & ○ & 0 & $\bullet$ & ० & 0 & ० & 0 & 0 & $\circ$ & ० & o & 0 & $\circ$ & $\bullet$ & 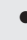 & & $\bullet$ \\
\hline \multicolumn{32}{|l|}{04030000} \\
\hline 04031000 & & & & & & & & & & & & & & & & & & & $\bullet$ & $\bullet$ & $\bullet$ & $\bullet$ & - & $\bullet$ & $\bullet$ & $\bullet$ & $\bullet$ & $\bullet$ & 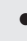 & & $\bullet$ \\
\hline \multicolumn{32}{|l|}{04031500} \\
\hline \multicolumn{32}{|l|}{04032000} \\
\hline \multicolumn{32}{|l|}{04032500} \\
\hline 04033000 & $\bullet$ & $\bullet$ & $\bullet$ & $\bullet$ & $\bullet$ & $\bullet$ & $\bullet$ & $\bullet$ & $\bullet$ & $\bullet$ & $\bullet$ & $\bullet$ & $\bullet$ & & & & & & $\bullet$ & $\bullet$ & $\bullet$ & $\bullet$ & $\bullet$ & $\bullet$ & $\bullet$ & $\bullet$ & $\bullet$ & $\bullet$ & 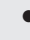 & & $\bullet$ \\
\hline 04033500 & 0 & 0 & 0 & 0 & $\circ$ & $\circ$ & $\circ$ & 0 & 0 & $\circ$ & o & 0 & 0 & $\circ$ & $\circ$ & 0 & 0 & 0 & 0 & $\circ$ & 0 & 0 & 0 & $\circ$ & 0 & 0 & 0 & $\circ$ & c & & 0 \\
\hline 04034500 & $\bullet$ & $\bullet$ & $\bullet$ & $\bullet$ & $\bullet$ & $\bullet$ & $\bullet$ & $\bullet$ & $\bullet$ & $\bullet$ & $\bullet$ & $\bullet$ & $\bullet$ & $\bullet$ & $\bullet$ & $\bullet$ & $\bullet$ & $\bullet$ & $\bullet$ & $\bullet$ & $\bullet$ & $\bullet$ & $\bullet$ & $\bullet$ & ० & o & $\circ$ & $\circ$ & & & \\
\hline \multicolumn{32}{|l|}{04035000} \\
\hline 04035500 & $\bullet$ & $\bullet$ & $\bullet$ & $\bullet$ & $\bullet$ & $\bullet$ & $\bullet$ & $\bullet$ & $\bullet$ & $\bullet$ & $\bullet$ & $\bullet$ & $\bullet$ & $\bullet$ & $\bullet$ & $\bullet$ & $\bullet$ & $\bullet$ & $\bullet$ & $\bullet$ & $\bullet$ & $\bullet$ & $\bullet$ & $\bullet$ & $\bullet$ & 0 & & & & & \\
\hline 04036000 & $\bullet$ & $\bullet$ & $\bullet$ & $\bullet$ & $\bullet$ & $\bullet$ & $\bullet$ & $\bullet$ & $\bullet$ & $\bullet$ & $\bullet$ & $\bullet$ & $\bullet$ & $\bullet$ & $\bullet$ & $\bullet$ & $\bullet$ & $\bullet$ & $\bullet$ & $\bullet$ & $\bullet$ & $\bullet$ & $\bullet$ & $\bullet$ & $\bullet$ & $\bullet$ & $\bullet$ & $\bullet$ & 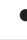 & & $\bullet$ \\
\hline 04037500 & - & - & - & - & - & - & - & - & - & - & - & - & - & - & - & $\bullet$ & - & • & • & $\bullet$ & - & - & - & • & $\bullet$ & - & - & - & . & & - \\
\hline \multicolumn{32}{|l|}{04039500} \\
\hline 04040000 & $\bullet$ & $\bullet$ & $\bullet$ & $\bullet$ & $\bullet$ & $\bullet$ & $\bullet$ & $\bullet$ & $\bullet$ & $\bullet$ & $\bullet$ & $\bullet$ & $\bullet$ & $\bullet$ & $\bullet$ & $\bullet$ & $\bullet$ & $\bullet$ & $\bullet$ & o & $\bullet$ & $\bullet$ & $\bullet$ & $\bullet$ & o & $\bullet$ & $\bullet$ & $\bullet$ & 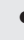 & & $\bullet$ \\
\hline 04040500 & $\bullet$ & $\bullet$ & $\bullet$ & $\bullet$ & $\bullet$ & $\bullet$ & $\bullet$ & $\bullet$ & $\bullet$ & $\bullet$ & $\bullet$ & $\bullet$ & $\bullet$ & $\bullet$ & $\bullet$ & $\bullet$ & $\bullet$ & $\bullet$ & $\bullet$ & $\bullet$ & $\bullet$ & $\bullet$ & $\bullet$ & $\bullet$ & $\bullet$ & $\bullet$ & $\bullet$ & $\bullet$ & 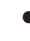 & & $\bullet$ \\
\hline 04041500 & $\bullet$ & $\bullet$ & $\bullet$ & $\bullet$ & $\bullet$ & $\bullet$ & $\bullet$ & $\bullet$ & $\bullet$ & $\bullet$ & $\bullet$ & $\bullet$ & $\bullet$ & $\bullet$ & $\bullet$ & $\bullet$ & $\bullet$ & $\bullet$ & $\bullet$ & $\bullet$ & $\bullet$ & $\bullet$ & $\bullet$ & $\bullet$ & $\bullet$ & $\bullet$ & $\bullet$ & $\bullet$ & 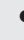 & & $\bullet$ \\
\hline \multicolumn{32}{|l|}{04042500} \\
\hline 04043000 & & & & & & & & & & & & & & & & & & & & & & & & & & & & & & & \\
\hline 04043050 & $\bullet$ & $\bullet$ & $\bullet$ & $\bullet$ & $\bullet$ & $\bullet$ & $\bullet$ & $\bullet$ & $\bullet$ & $\bullet$ & $\bullet$ & $\bullet$ & $\bullet$ & $\bullet$ & $\bullet$ & $\bullet$ & $\bullet$ & $\bullet$ & $\bullet$ & $\bullet$ & $\bullet$ & $\bullet$ & $\bullet$ & $\bullet$ & $\bullet$ & $\bullet$ & $\bullet$ & $\bullet$ & 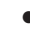 & & $\bullet$ \\
\hline 04043140 & & & & & & & & & & & & & & & & & & & & & & & & & & 0 & $\circ$ & $\circ$ & $c$ & & 0 \\
\hline 04043150 & & & & & & & & & & & & & & & & & & & & $\bullet$ & $\bullet$ & $\bullet$ & $\bullet$ & $\bullet$ & $\bullet$ & $\bullet$ & $\bullet$ & $\bullet$ & 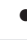 & & $\bullet$ \\
\hline 04043238 & & & & & & & & & & & & & & & & & & & & & & & & $\circ$ & 0 & 0 & 0 & $\circ$ & c & & 0 \\
\hline 04043244 & & & & & & & & & & & & & & & & & & & & & & & & ○ & o & 0 & ० & $\circ$ & c & & o \\
\hline 04043275 & & & & & & & & & & & & & & & & & & & & & & & & o & o & 0 & ० & $\circ$ & $c$ & & 0 \\
\hline 04043800 & & & & & & & & & $\circ$ & $\circ$ & $\circ$ & o & o & $\circ$ & ○ & o & o & ० & o & $\circ$ & o & & $\circ$ & $\circ$ & o & & & & & & \\
\hline 04044400 & $\bullet$ & $\bullet$ & $\bullet$ & $\bullet$ & & & & & & & & & & & & & & & & & & & & & & & & & & & \\
\hline 04044563 & & & & & & & & & & & & & & & & & & & & & & & & & & & & & & & \\
\hline 04044573 & & & & & & & & & & & & & & & & & & & & & & & & & & & & & & & \\
\hline 04044583 & & & & & & & & & & & & & & & & & & & & & & & & & & & & & & & \\
\hline 04044595 & & & & & & & & & & & & & & & & & & & & & & & & & & & & & & & \\
\hline 04044724 & & & & & & & & & & & & $\bullet$ & $\bullet$ & $\bullet$ & $\bullet$ & $\bullet$ & $\bullet$ & 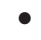 & $\bullet$ & $\bullet$ & $\bullet$ & $\bullet$ & $\bullet$ & $\bullet$ & $\bullet$ & $\bullet$ & $\bullet$ & $\bullet$ & 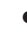 & & $\bullet$ \\
\hline 04045500 & - & - & - & - & - & - & - & - & - & $\bullet$ & - & - & - & - & - & - & - & • & - & $\bullet$ & $\bullet$ & - & - & - & - & $\bullet$ & - & - & r & & • \\
\hline
\end{tabular}


Table 3. Selected explanatory variables for each study area.

[PRISM, Parameter-elevation Regressions on Independent Slopes Model; NLCD, National Land Cover Data; SSURGO, Soil Survey Geography; GAP, USGS National Gap Analysis Program; GDD, growing degree days]

\begin{tabular}{|c|c|c|c|c|c|c|c|}
\hline Explanatory variables & $\begin{array}{l}\text { Study } \\
\text { area } 1\end{array}$ & $\begin{array}{c}\text { Study } \\
\text { area } 2\end{array}$ & $\begin{array}{c}\text { Study } \\
\text { area } 3\end{array}$ & $\begin{array}{c}\text { Study } \\
\text { area } 4\end{array}$ & $\begin{array}{c}\text { Study } \\
\text { area } 5\end{array}$ & $\begin{array}{c}\text { Study } \\
\text { area } 6\end{array}$ & $\begin{array}{c}\text { Study } \\
\text { area } 7\end{array}$ \\
\hline \multicolumn{8}{|c|}{ PRISM } \\
\hline Preced precip, in hundredths of millimeters & $\mathrm{X}$ & $\mathrm{X}$ & $\mathrm{X}$ & $\mathrm{X}$ & $\mathrm{X}$ & $\mathrm{X}$ & $\mathrm{X}$ \\
\hline Current temp, in degrees Celsius $* 100$ & $\mathrm{X}$ & $\mathrm{X}$ & $\mathrm{X}$ & $\mathrm{X}$ & $X$ & $\mathrm{X}$ & $X$ \\
\hline Developed, in percent & & & & & & & $\mathrm{X}$ \\
\hline Deciduous and evergreen forest, in percent & & & & $\mathrm{X}$ & & & \\
\hline Forest, in percent & $\mathrm{X}$ & $\mathrm{X}$ & $\mathrm{X}$ & & & $\mathrm{X}$ & $\mathrm{X}$ \\
\hline Grass herbaceous, in percent & & & & & $\mathrm{X}$ & & \\
\hline Pasture hay and row crops, in percent & & $\mathrm{X}$ & & & & $\mathrm{X}$ & $\mathrm{X}$ \\
\hline Wetland woody, in percent & & $X$ & & & & & \\
\hline Wetland herbaceous, in percent & & $\mathrm{X}$ & $\mathrm{X}$ & & & & \\
\hline \multicolumn{8}{|c|}{ NHDPlus catchment characteristics } \\
\hline Stream order, dimensionless & $\mathrm{X}$ & & $\mathrm{X}$ & $\mathrm{X}$ & $\mathrm{X}$ & $\mathrm{X}$ & \\
\hline Mean elevation, in centimeters & $\mathrm{X}$ & & $\mathrm{X}$ & & & $\mathrm{X}$ & \\
\hline Mean slope, in centimeters/meter & & & $\mathrm{X}$ & & $X$ & $\mathrm{X}$ & $\mathrm{X}$ \\
\hline \multicolumn{8}{|c|}{ SSURGO } \\
\hline Hydric group A and AD, in percent & & & & $\mathrm{X}$ & & & \\
\hline Fine end and ground moraines, in percent & & & & & & $\mathrm{X}$ & \\
\hline Medium end and ground moraines, in percent & & & & & & $\mathrm{X}$ & \\
\hline Coarse end and ground moraines, in percent & $\mathrm{X}$ & & & & & & $\mathrm{X}$ \\
\hline Coarse outwash and ice contact, in percent & & & & & & $\mathrm{X}$ & $X$ \\
\hline Coarse stratified sediment, in percent & & $\mathrm{X}$ & & & & & \\
\hline Till, in percent & & $\mathrm{X}$ & & & & & \\
\hline \multicolumn{8}{|c|}{ GDD } \\
\hline Average GDD, in days & & & $\mathrm{X}$ & & $\mathrm{X}$ & & $\mathrm{X}$ \\
\hline
\end{tabular}


Table 4. Regression statistics and explanatory variables used in the AFINCH (Analysis of Flows In Networks of Channels) analysis of flows in study area 1 (hydrologic subregions 0401 and 0402), in the U.S. Great Lakes Basin, by month.

[-, indicates variable not included in monthly regression equation; RMSE, root mean square error; $\mathrm{R}^{2}$, coefficient of determination. See appendix tables for variable descriptions]

\begin{tabular}{|c|c|c|c|c|c|c|c|c|c|c|c|c|}
\hline \multirow[b]{2}{*}{ Month } & \multicolumn{9}{|c|}{ Regression coefficients } & \multicolumn{3}{|c|}{ Regression fit statistics } \\
\hline & Intercept & $\begin{array}{c}\text { Current } \\
\text { precipitation }\end{array}$ & $\begin{array}{c}\text { Preceding } \\
\text { precipitation }\end{array}$ & $\begin{array}{c}\text { Current } \\
\text { temperature }\end{array}$ & Forest & $\begin{array}{l}\text { Coarse } \\
\text { end and } \\
\text { ground } \\
\text { moraines }\end{array}$ & $\begin{array}{c}\text { Mean } \\
\text { elevation }\end{array}$ & $\begin{array}{c}\text { Hydric } \\
\text { groups C, } \\
\text { CD, and D }\end{array}$ & $\begin{array}{c}\text { Stream } \\
\text { order }\end{array}$ & RMSE & F-statistic & $\mathbf{R}^{2}$ \\
\hline October & 0.797 & 0.116 & 0.080 & - & - & - & - & -0.326 & -0.203 & 0.236 & 391.3 & 0.537 \\
\hline November & 1.133 & 0.120 & 0.068 & -0.011 & - & 0.073 & $-6.0 \mathrm{E}-06$ & -0.379 & -0.180 & 0.221 & 144.4 & 0.428 \\
\hline December & 1.314 & 0.060 & 0.062 & 0.015 & 0.113 & 0.145 & $-7.0 \mathrm{E}-06$ & -0.496 & -0.158 & 0.184 & 144.4 & 0.462 \\
\hline January & 1.279 & 0.027 & 0.054 & 0.008 & 0.225 & 0.193 & $-9.0 \mathrm{E}-06$ & -0.547 & -0.132 & 0.192 & 124.3 & 0.425 \\
\hline February & 1.261 & - & 0.049 & 0.015 & 0.307 & 0.196 & $-9.0 \mathrm{E}-06$ & -0.498 & -0.111 & 0.193 & 125.7 & 0.395 \\
\hline March & 0.926 & 0.091 & 0.047 & 0.068 & 0.681 & 0.074 & $-7.0 \mathrm{E}-06$ & - & - & 0.254 & 248.5 & 0.525 \\
\hline April & 0.395 & 0.101 & 0.089 & -0.036 & 0.887 & -0.285 & - & 0.709 & 0.278 & 0.465 & 62.1 & 0.244 \\
\hline May & 1.064 & 0.146 & 0.066 & -0.048 & - & -0.262 & - & -0.121 & 0.169 & 0.343 & 137.1 & 0.379 \\
\hline June & 0.818 & 0.133 & 0.071 & -0.016 & - & -0.134 & $-4.0 \mathrm{E}-06$ & -0.241 & - & 0.246 & 184 & 0.450 \\
\hline July & 1.296 & 0.123 & 0.061 & -0.037 & 0.150 & - & $-1.0 \mathrm{E}-05$ & -0.385 & - & 0.225 & 263.7 & 0.540 \\
\hline August & 0.044 & 0.086 & 0.060 & 0.028 & 0.290 & - & $-9.0 \mathrm{E}-06$ & -0.470 & - & 0.217 & 213 & 0.486 \\
\hline September & 0.781 & 0.100 & 0.064 & - & 0.156 & - & $-6.0 \mathrm{E}-06$ & -0.458 & -0.158 & 0.222 & 229.5 & 0.505 \\
\hline
\end{tabular}




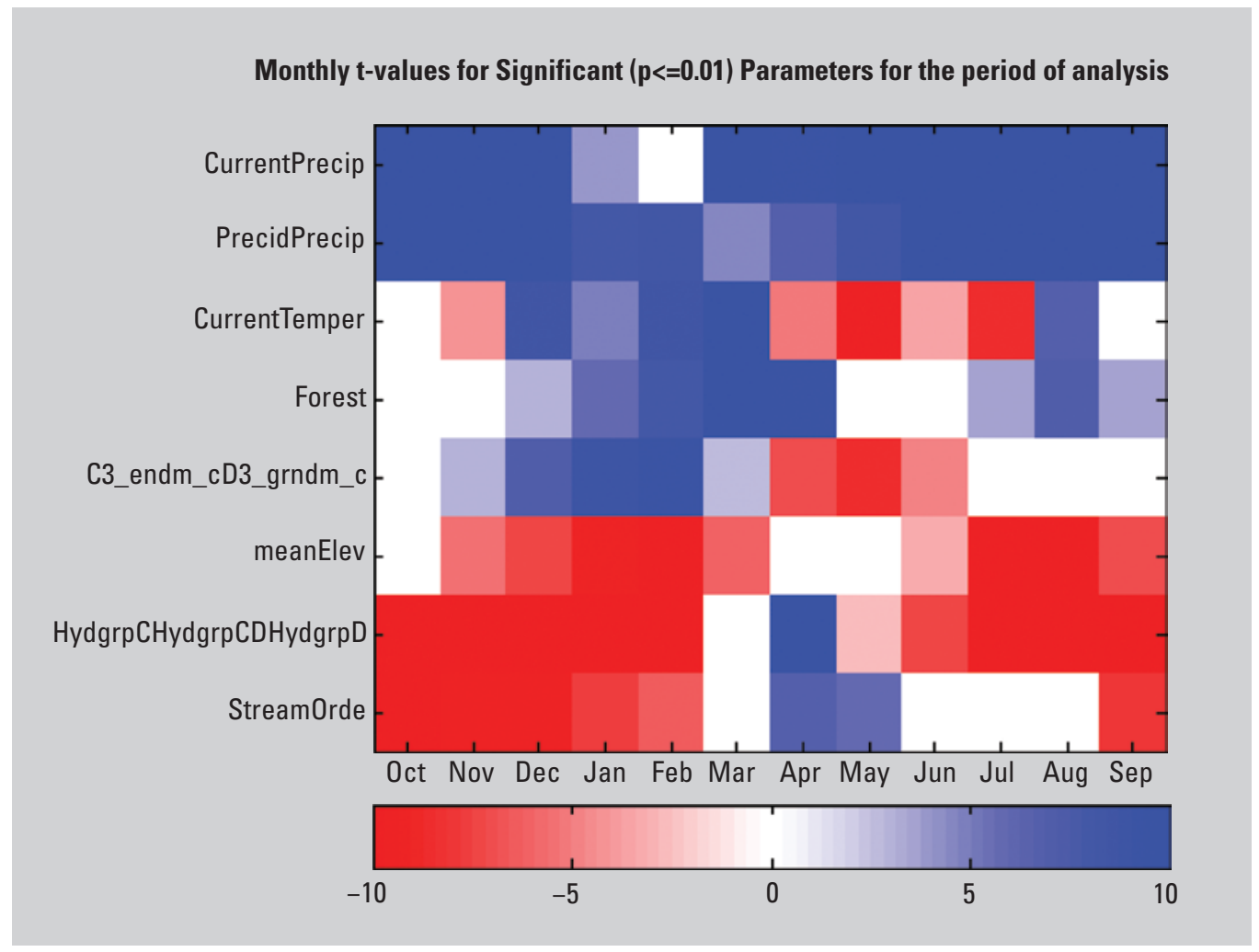

Figure 5. Image showing the t-value-indexed colors corresponding to each explanatory variable in the monthly regression equation for estimating water yield for study area 1 (hydrologic subregions 0401 and 0402) in the U.S. Great Lakes Basin. 


\section{Study Area 2}

Study area 2 is formed by hydrologic subregion 0403 and cataloguing units 04040002 , 04040003, 04060106, 04060107, 04070001, and 04070002 on the western and northern side of Lake Michigan and encompasses parts of Wisconsin, northern Illinois, and the Upper Peninsula of Michigan (fig. 6). The number of stations included in the analysis ranged from
21 (1951 and 1953) to 47 (2005) (table 5). Ten explanatory variables were selected during the regression analysis with the number of variables specified for each monthly equation ranging from 6 (March and June) to 10 (February) (table 3). Variables and regression statistics based on the stepwise regression for each month are indicated in table 6 and figure 7.

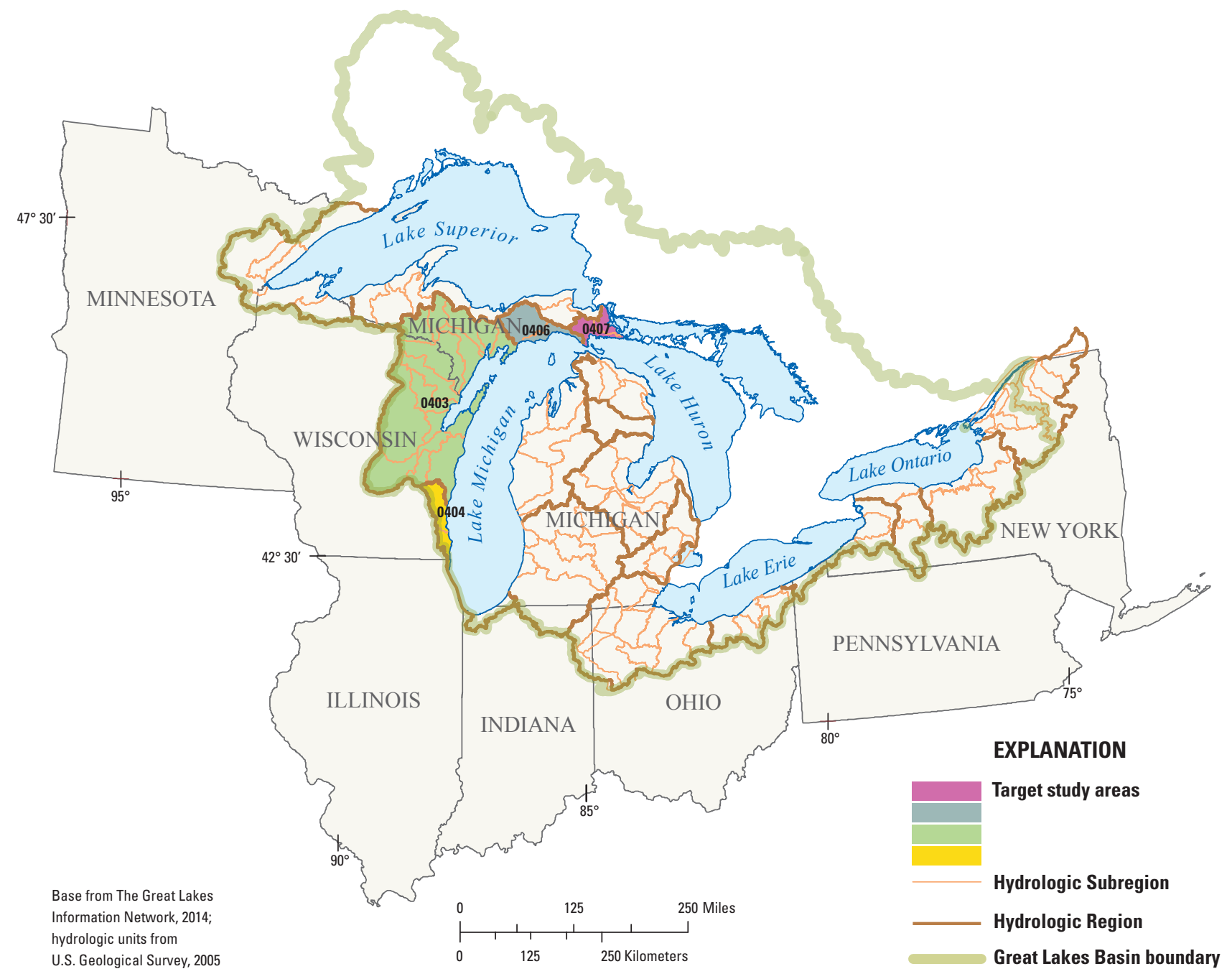

Figure 6. Location of study area 2 (hydrologic subregion 0403 and cataloguing units 04040002, 04040003, 04060106, 04060107, 04070001, and 04070002) in the U.S. Great Lakes Basin. 
Table 5. Streamgaging stations considered for analysis of study area 2 (hydrologic subregion 0403 and cataloguing units 04040002,04040003 , 04060106, 04060107, 04070001, and 04070002) in the U.S. Great Lakes Basin, by water year.

[ $\bullet$, station active and included in the analysis; $\circ$, station active but not included in the analysis]

\begin{tabular}{|c|c|c|c|c|c|c|c|c|c|c|c|c|c|c|c|c|c|c|c|c|c|c|c|c|c|c|c|c|c|c|c|c|}
\hline \multirow{2}{*}{$\begin{array}{l}\text { Station } \\
\text { number }\end{array}$} & \multicolumn{32}{|c|}{ Water year } \\
\hline & ్ㅐㅇ & ్ㅗㅇ & 艿 & 壱 & 낭 & 突 & 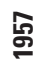 & 哭 & 吕 & চ్ర్ & б్ & ช్ర & ஜூ & 芯 & 농 & ஜ্ & ஜ্ & ஜே & 양 & 윰 & চ & న్ & $\stackrel{\Re}{\sigma}$ & 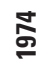 & 占 & $\stackrel{\circ}{\circ}$ & E & $\stackrel{\infty}{\sigma}$ & হ̊. & 喿 & চ্ & ঞ \\
\hline 04046000 & & $\bullet$ & $\bullet$ & $\bullet$ & $\bullet$ & $\bullet$ & $\bullet$ & $\bullet$ & $\bullet$ & $\bullet$ & $\bullet$ & $\bullet$ & $\bullet$ & $\bullet$ & $\bullet$ & $\bullet$ & $\bullet$ & $\bullet$ & $\bullet$ & $\bullet$ & $\bullet$ & $\bullet$ & $\bullet$ & $\bullet$ & $\bullet$ & $\bullet$ & $\bullet$ & $\bullet$ & & & & \\
\hline 04049500 & $\bullet$ & $\bullet$ & $\bullet$ & $\bullet$ & $\bullet$ & $\bullet$ & $\bullet$ & $\bullet$ & $\bullet$ & $\bullet$ & $\bullet$ & $\bullet$ & $\bullet$ & $\bullet$ & $\bullet$ & $\bullet$ & $\bullet$ & $\bullet$ & $\bullet$ & $\bullet$ & & & & & & & & & & & & \\
\hline \multicolumn{33}{|l|}{04052500} \\
\hline \multicolumn{33}{|l|}{04052600} \\
\hline 04054500 & $\bullet$ & $\bullet$ & $\bullet$ & $\bullet$ & & & & & & & & & & & & & & & & & & & & & & & & & & & & \\
\hline 04055000 & $\bullet$ & $\bullet$ & $\bullet$ & $\bullet$ & $\bullet$ & $\bullet$ & $\bullet$ & $\bullet$ & $\bullet$ & $\bullet$ & $\bullet$ & $\bullet$ & $\bullet$ & $\bullet$ & $\bullet$ & $\bullet$ & $\bullet$ & $\bullet$ & $\bullet$ & $\bullet$ & & & & & & & & & & & & \\
\hline 04056000 & $\bullet$ & $\bullet$ & $\bullet$ & $\bullet$ & $\bullet$ & $\bullet$ & & & & & & & & & & & & & & & & & & & & & & & & & & \\
\hline 04056500 & $\circ$ & $\bullet$ & $\circ$ & $\bullet$ & $\bullet$ & $\circ$ & $\bullet$ & $\bullet$ & $\bullet$ & $\bullet$ & $\bullet$ & $\bullet$ & $\bullet$ & $\bullet$ & $\bullet$ & $\bullet$ & $\bullet$ & $\bullet$ & $\bullet$ & $\bullet$ & $\bullet$ & $\bullet$ & $\bullet$ & $\bullet$ & $\bullet$ & $\bullet$ & $\bullet$ & $\bullet$ & $\bullet$ & $\bullet$ & $\bullet$ & $\bullet$ \\
\hline 04057000 & $\bullet$ & $\bullet$ & $\bullet$ & $\bullet$ & $\bullet$ & $\bullet$ & $\bullet$ & $\bullet$ & $\bullet$ & $\bullet$ & $\bullet$ & $\bullet$ & $\bullet$ & $\bullet$ & $\bullet$ & $\bullet$ & $\bullet$ & $\bullet$ & $\bullet$ & $\bullet$ & $\bullet$ & & & & & & & & & & & \\
\hline \multicolumn{33}{|l|}{04057004} \\
\hline 04057510 & & & & & & & & & & & & & & & & & $\bullet$ & $\bullet$ & $\bullet$ & $\bullet$ & $\bullet$ & $\bullet$ & $\bullet$ & $\bullet$ & $\bullet$ & $\bullet$ & $\bullet$ & $\bullet$ & $\bullet$ & $\bullet$ & $\bullet$ & $\bullet$ \\
\hline 04057800 & & & & & & & & & & $\bullet$ & $\bullet$ & $\bullet$ & $\bullet$ & $\bullet$ & $\bullet$ & $\bullet$ & $\bullet$ & $\bullet$ & $\bullet$ & $\bullet$ & $\bullet$ & $\bullet$ & $\bullet$ & $\bullet$ & $\bullet$ & $\bullet$ & $\bullet$ & $\bullet$ & $\bullet$ & $\bullet$ & $\bullet$ & $\bullet$ \\
\hline 04057813 & & & & & & & & & & & & & & & & & & & & & & & & $\circ$ & $\circ$ & $\circ$ & ○ & $\circ$ & $\circ$ & ○ & O & ○ \\
\hline 04057814 & & & & & & & & & & & & & & & & & & & & & & & $\circ$ & $\circ$ & $\circ$ & $\circ$ & $\circ$ & $\circ$ & $\circ$ & $\circ$ & $\circ$ & ○ \\
\hline 04057820 & & & & & & & & & & & & & & & & & & & & & & & $\bullet$ & $\bullet$ & $\bullet$ & $\bullet$ & $\bullet$ & $\bullet$ & $\bullet$ & $\bullet$ & $\bullet$ & $\bullet$ \\
\hline 04057900 & & & & & & & & & & & & $\bullet$ & $\bullet$ & $\bullet$ & $\bullet$ & $\bullet$ & $\bullet$ & $\bullet$ & & & & & & & & & & & & & & \\
\hline 04058000 & & & & & $\bullet$ & $\bullet$ & $\bullet$ & $\bullet$ & $\bullet$ & $\bullet$ & $\bullet$ & $\bullet$ & $\bullet$ & $\bullet$ & $\bullet$ & $\bullet$ & $\bullet$ & $\bullet$ & $\bullet$ & $\bullet$ & $\bullet$ & $\bullet$ & $\bullet$ & $\bullet$ & $\bullet$ & & & & & & & \\
\hline 04058100 & & & & & & & & & & & & $\bullet$ & $\bullet$ & $\bullet$ & $\bullet$ & $\bullet$ & $\bullet$ & $\bullet$ & $\bullet$ & $\bullet$ & $\bullet$ & $\bullet$ & $\bullet$ & $\bullet$ & $\bullet$ & $\bullet$ & $\bullet$ & $\bullet$ & $\bullet$ & $\bullet$ & $\bullet$ & $\bullet$ \\
\hline 04058130 & & & & & & & & & & & & & & & & & & & & & & & & & & & $\bullet$ & $\bullet$ & $\bullet$ & $\bullet$ & $\bullet$ & $\bullet$ \\
\hline 04058200 & & & & & & & & & & & $\bullet$ & $\bullet$ & $\bullet$ & $\bullet$ & $\bullet$ & $\bullet$ & $\bullet$ & $\bullet$ & $\bullet$ & $\bullet$ & $\bullet$ & $\bullet$ & $\bullet$ & $\bullet$ & $\bullet$ & $\bullet$ & $\bullet$ & $\bullet$ & $\bullet$ & $\bullet$ & $\bullet$ & $\bullet$ \\
\hline 04058300 & & & & & & & & & & & & $\bullet$ & $\bullet$ & $\bullet$ & $\bullet$ & $\bullet$ & $\bullet$ & $\bullet$ & & & & & $\bullet$ & $\bullet$ & $\bullet$ & $\bullet$ & $\bullet$ & $\bullet$ & & & & \\
\hline 04058400 & & & & & & & & & & & & & & & & $\bullet$ & $\bullet$ & $\bullet$ & $\bullet$ & $\bullet$ & $\bullet$ & $\bullet$ & $\bullet$ & $\bullet$ & $\bullet$ & $\bullet$ & $\bullet$ & $\bullet$ & $\bullet$ & $\bullet$ & $\bullet$ & $\bullet$ \\
\hline 04058500 & & & & & $\bullet$ & $\bullet$ & $\bullet$ & $\bullet$ & $\bullet$ & $\bullet$ & $\bullet$ & $\bullet$ & $\bullet$ & $\bullet$ & $\bullet$ & $\bullet$ & $\bullet$ & $\bullet$ & $\bullet$ & $\bullet$ & $\bullet$ & $\bullet$ & $\bullet$ & $\bullet$ & $\bullet$ & $\bullet$ & $\bullet$ & $\bullet$ & $\bullet$ & $\bullet$ & & \\
\hline 04059000 & $\circ$ & $\circ$ & $\circ$ & $\circ$ & $\circ$ & $\circ$ & $\circ$ & $\circ$ & $\circ$ & $\circ$ & $\circ$ & 0 & $\circ$ & $\circ$ & ० & $\circ$ & $\circ$ & $\circ$ & $\circ$ & $\circ$ & ○ & o & ○ & $\circ$ & ○ & ○ & $\circ$ & $\circ$ & $\circ$ & 0 & $\circ$ & ○ \\
\hline 04059400 & & & & & & & & & & & & & & & & & & & & & & $\bullet$ & $\bullet$ & $\bullet$ & $\bullet$ & $\bullet$ & $\bullet$ & & & & & \\
\hline 04059500 & & & & & $\bullet$ & $\bullet$ & $\bullet$ & $\bullet$ & $\bullet$ & $\bullet$ & $\bullet$ & $\bullet$ & $\bullet$ & $\bullet$ & $\bullet$ & $\bullet$ & $\bullet$ & $\bullet$ & $\bullet$ & $\bullet$ & $\bullet$ & $\bullet$ & $\bullet$ & $\bullet$ & $\bullet$ & $\bullet$ & $\bullet$ & $\bullet$ & $\bullet$ & $\bullet$ & $\bullet$ & $\bullet$ \\
\hline 04060500 & $\bullet$ & 0 & $\circ$ & $\circ$ & o & $\circ$ & $\circ$ & ० & 0 & $\circ$ & $\circ$ & o & ० & o & 0 & $\circ$ & 0 & o & o & 0 & $\circ$ & 0 & 0 & $\circ$ & ० & o & $\circ$ & $\circ$ & $\bullet$ & $\bullet$ & & \\
\hline 04060993 & $\circ$ & $\circ$ & $\circ$ & 0 & 0 & 0 & $\circ$ & 0 & 0 & 0 & $\circ$ & 0 & $\circ$ & 0 & $\circ$ & $\circ$ & $\circ$ & ० & o & $\circ$ & $\circ$ & o & $\circ$ & $\circ$ & $\circ$ & ○ & $\circ$ & $\circ$ & o & o & $\circ$ & ○ \\
\hline 04061000 & $\bullet$ & $\bullet$ & $\bullet$ & $\bullet$ & $\bullet$ & $\bullet$ & $\bullet$ & $\bullet$ & $\bullet$ & $\bullet$ & $\bullet$ & $\bullet$ & $\bullet$ & $\bullet$ & $\bullet$ & $\bullet$ & $\bullet$ & $\bullet$ & $\bullet$ & $\bullet$ & $\bullet$ & $\bullet$ & $\bullet$ & $\bullet$ & $\bullet$ & $\bullet$ & $\bullet$ & $\bullet$ & $\bullet$ & $\bullet$ & $\bullet$ & $\bullet$ \\
\hline 04061500 & 0 & o & $\circ$ & $\circ$ & $\circ$ & $\circ$ & $\circ$ & $\circ$ & $\circ$ & $\circ$ & $\circ$ & o & $\circ$ & $\circ$ & $\circ$ & $\circ$ & $\circ$ & $\circ$ & $\circ$ & $\circ$ & $\circ$ & o & $\circ$ & $\circ$ & $\circ$ & $\circ$ & $\circ$ & $\circ$ & $\circ$ & o & $\circ$ & $\circ$ \\
\hline 04062000 & & & $\circ$ & $\circ$ & $\circ$ & $\circ$ & $\circ$ & $\circ$ & $\circ$ & $\circ$ & 0 & ○ & $\circ$ & $\circ$ & ○ & $\circ$ & $\circ$ & ○ & $\circ$ & 0 & $\circ$ & o & $\circ$ & ○ & o & $\circ$ & $\circ$ & ० & $\circ$ & ० & 0 & ○ \\
\hline \multicolumn{33}{|l|}{04062011} \\
\hline 04062100 & & & & & & & & & & & & $\bullet$ & $\bullet$ & $\bullet$ & $\bullet$ & $\bullet$ & $\bullet$ & $\bullet$ & & & & & & & & & & & & & & \\
\hline 04062200 & & & & & & & & & & & & $\bullet$ & $\bullet$ & $\bullet$ & $\bullet$ & $\bullet$ & $\bullet$ & $\bullet$ & $\bullet$ & $\bullet$ & $\bullet$ & $\bullet$ & $\bullet$ & $\bullet$ & $\bullet$ & $\bullet$ & $\bullet$ & $\bullet$ & & & & \\
\hline 04062230 & & & & & & & & & & & & & & & & & & & 0 & 0 & o & o & $\circ$ & $\circ$ & $\circ$ & $\circ$ & 0 & o & o & 0 & 0 & ० \\
\hline 04062270 & & & & & & & & & & & & & & & $\circ$ & $\circ$ & 0 & $\circ$ & $\circ$ & & & & & & & & & & & & & \\
\hline 04062300 & & & & & & & & & & & & o & $\circ$ & $\circ$ & ○ & $\circ$ & $\circ$ & ○ & $\circ$ & $\circ$ & o & o & o & $\circ$ & o & & & & & & & \\
\hline 04062400 & & & & & & & & & & & & & & & $\bullet$ & $\bullet$ & $\bullet$ & $\circ$ & $\bullet$ & $\circ$ & $\bullet$ & $\bullet$ & $\bullet$ & $\bullet$ & $\bullet$ & $\bullet$ & $\bullet$ & $\bullet$ & $\bullet$ & $\bullet$ & & \\
\hline 04062500 & ○ & 0 & $\circ$ & $\circ$ & $\circ$ & $\circ$ & $\circ$ & $\circ$ & $\circ$ & $\circ$ & 0 & ○ & $\circ$ & $\circ$ & ○ & $\circ$ & $\circ$ & ○ & $\circ$ & 0 & ○ & o & $\circ$ & ○ & ० & $\circ$ & $\circ$ & ० & $\circ$ & ० & ○ & ○ \\
\hline 04063000 & $\circ$ & $\circ$ & $\circ$ & $\circ$ & $\circ$ & $\circ$ & $\circ$ & $\circ$ & $\circ$ & $\circ$ & 0 & $\circ$ & $\circ$ & $\circ$ & ० & $\circ$ & $\circ$ & $\circ$ & $\circ$ & $\circ$ & $\circ$ & o & $\circ$ & $\circ$ & ○ & $\circ$ & $\circ$ & $\circ$ & $\circ$ & $\circ$ & $\circ$ & ○ \\
\hline 04063500 & ○ & $\circ$ & 0 & 0 & 0 & $\circ$ & $\circ$ & $\circ$ & 0 & $\circ$ & 0 & 0 & 0 & 0 & 0 & $\circ$ & 0 & 0 & 0 & 0 & 0 & 0 & $\circ$ & 0 & 0 & 0 & 0 & 0 & 0 & 0 & 0 & 0 \\
\hline 04063640 & & & & & & & & & & & & & & & & & $\bullet$ & & & & & & & & & & & & & & & \\
\hline 04063700 & & & & & & & & & & & & & & $\bullet$ & $\bullet$ & $\bullet$ & $\bullet$ & $\bullet$ & $\bullet$ & $\bullet$ & $\bullet$ & $\bullet$ & $\bullet$ & $\bullet$ & $\bullet$ & $\bullet$ & $\bullet$ & $\bullet$ & $\bullet$ & $\bullet$ & $\bullet$ & $\bullet$ \\
\hline 04064500 & $\bullet$ & $\bullet$ & $\bullet$ & $\bullet$ & $\bullet$ & $\bullet$ & $\bullet$ & $\bullet$ & $\bullet$ & $\bullet$ & $\bullet$ & $\bullet$ & $\bullet$ & $\bullet$ & $\bullet$ & $\bullet$ & $\bullet$ & $\bullet$ & $\bullet$ & $\bullet$ & $\bullet$ & $\bullet$ & $\bullet$ & $\bullet$ & $\bullet$ & & & & & & & \\
\hline
\end{tabular}


Table 5. Streamgaging stations considered for analysis of study area 2 (hydrologic subregion 0403 and cataloguing units 04040002 , 04040003, 04060106, 04060107, 04070001, and 04070002) in the U.S. Great Lakes Basin, by water year.-Continued

[ $\bullet$, station active and included in the analysis; $\circ$, station active but not included in the analysis]

\begin{tabular}{|c|c|c|c|c|c|c|c|c|c|c|c|c|c|c|c|c|c|c|c|c|c|c|c|c|c|c|c|c|c|c|}
\hline \multirow{2}{*}{$\begin{array}{l}\text { Station } \\
\text { number }\end{array}$} & \multicolumn{30}{|c|}{ Water year } \\
\hline & $\underset{\mathscr{g}}{\mathscr{\sigma}}$ & 品 & 棁 & $\stackrel{\mathscr{g}}{\circ}$ & ळ్ & 怘 & 吕 & 足 & চ & §ু & ஜू & 苂 & 농 & 足 & ஓ & 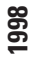 & 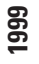 & ర్ & ర్స & ণ్రి & 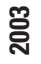 & 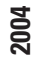 & 농 & 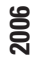 & ్ㅗㅇ & 号 & 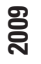 & 옴 & $\overline{\text { స్ }}$ & 국 \\
\hline 04046000 & & & & & & & & & & & & & $\bullet$ & $\bullet$ & $\bullet$ & $\bullet$ & $\bullet$ & $\bullet$ & $\bullet$ & $\bullet$ & $\bullet$ & $\bullet$ & $\bullet$ & $\bullet$ & $\bullet$ & $\bullet$ & $\bullet$ & $\bullet$ & $\bullet$ & $\bullet$ \\
\hline \multicolumn{31}{|l|}{04049500} \\
\hline 04052500 & & & & & & & & & & & & & & & & & & & & & $\bullet$ & & & & & & & & & \\
\hline 04052600 & & & & & & & & & & & & & & & & & & & & & $\circ$ & & & & & & & & & \\
\hline \multicolumn{31}{|l|}{04054500} \\
\hline \multicolumn{31}{|l|}{04055000} \\
\hline \multicolumn{31}{|l|}{04056000} \\
\hline 04056500 & $\bullet$ & $\bullet$ & $\bullet$ & $\bullet$ & $\bullet$ & $\bullet$ & $\bullet$ & $\bullet$ & $\bullet$ & $\bullet$ & $\bullet$ & $\bullet$ & $\bullet$ & $\bullet$ & $\bullet$ & $\bullet$ & $\bullet$ & $\bullet$ & $\bullet$ & $\bullet$ & $\bullet$ & $\bullet$ & $\bullet$ & $\bullet$ & $\bullet$ & $\bullet$ & $\bullet$ & $\bullet$ & $\bullet$ & $\bullet$ \\
\hline 04057000 & & & & & & & & & & & $\bullet$ & & & & & & & & & & & & & & & & & & & \\
\hline 04057004 & & & & & & & & & & & & & $\bullet$ & & & & & & & & & & & & & & & & & \\
\hline 04057510 & $\bullet$ & $\bullet$ & $\bullet$ & $\bullet$ & $\bullet$ & $\bullet$ & $\bullet$ & $\bullet$ & $\bullet$ & $\bullet$ & $\bullet$ & $\bullet$ & $\bullet$ & $\bullet$ & $\bullet$ & $\bullet$ & $\bullet$ & $\bullet$ & $\bullet$ & $\bullet$ & $\bullet$ & $\bullet$ & $\bullet$ & $\bullet$ & $\bullet$ & $\bullet$ & $\bullet$ & $\bullet$ & $\bullet$ & $\bullet$ \\
\hline 04057800 & $\bullet$ & $\bullet$ & $\bullet$ & $\bullet$ & $\bullet$ & $\bullet$ & $\bullet$ & $\bullet$ & $\bullet$ & $\bullet$ & $\bullet$ & $\bullet$ & $\bullet$ & $\bullet$ & $\bullet$ & $\bullet$ & $\bullet$ & $\bullet$ & $\bullet$ & $\bullet$ & $\bullet$ & $\bullet$ & $\bullet$ & $\bullet$ & $\bullet$ & $\bullet$ & $\bullet$ & $\bullet$ & $\bullet$ & - \\
\hline 04057813 & ० & 0 & 0 & 0 & 0 & ○ & $\circ$ & 0 & 0 & 0 & o & 0 & 0 & o & O & 0 & $\circ$ & 0 & 0 & 0 & $\circ$ & 0 & 0 & 0 & 0 & 0 & 0 & 0 & 0 & 0 \\
\hline 04057814 & o & o & O & O & $\circ$ & 0 & $\circ$ & 0 & 0 & 0 & $\circ$ & $\circ$ & O & O & $\circ$ & O & $\circ$ & 0 & 0 & 0 & $\circ$ & 0 & O & O & 0 & $\circ$ & $\circ$ & 0 & 0 & O \\
\hline \multicolumn{31}{|l|}{04057820} \\
\hline \multicolumn{31}{|l|}{04057900} \\
\hline \multicolumn{31}{|l|}{04058000} \\
\hline 04058100 & & & & & & & & $\bullet$ & $\bullet$ & $\bullet$ & $\bullet$ & $\bullet$ & $\bullet$ & $\bullet$ & $\bullet$ & $\bullet$ & $\bullet$ & $\bullet$ & $\bullet$ & $\bullet$ & $\bullet$ & $\bullet$ & $\bullet$ & $\bullet$ & $\bullet$ & $\bullet$ & $\bullet$ & $\bullet$ & $\bullet$ & $\bullet$ \\
\hline \multicolumn{31}{|l|}{04058130} \\
\hline 04058200 & $\bullet$ & $\bullet$ & $\bullet$ & $\bullet$ & $\bullet$ & $\bullet$ & $\bullet$ & $\bullet$ & $\bullet$ & $\bullet$ & $\bullet$ & $\bullet$ & $\bullet$ & $\bullet$ & $\bullet$ & $\bullet$ & $\bullet$ & $\bullet$ & $\bullet$ & $\bullet$ & $\bullet$ & $\bullet$ & $\bullet$ & $\bullet$ & $\bullet$ & $\bullet$ & $\bullet$ & 0 & $\circ$ & 0 \\
\hline 04058300 & & & & & & & & & & & & & & & & & & & & & & & & & & & & & & \\
\hline 04058400 & & & & & & & & & & & & & & & & & & & & & & & & & & & & & & \\
\hline 04058500 & & & & & & & & & & & & & & & & & & & & & & & & & & & & & & \\
\hline 04059000 & 0 & ○ & O & 0 & 0 & 0 & $\circ$ & 0 & 0 & 0 & $\circ$ & 0 & O & 0 & $\circ$ & O & $\circ$ & 0 & ○ & 0 & $\circ$ & 0 & O & O & 0 & $\circ$ & $\circ$ & $\circ$ & 0 & 0 \\
\hline 04059400 & & & & & & & & & & & & & & & & & & & & & & & & & & & & & & \\
\hline 04059500 & $\bullet$ & $\bullet$ & $\bullet$ & $\bullet$ & $\bullet$ & $\bullet$ & $\bullet$ & $\bullet$ & $\bullet$ & $\bullet$ & $\bullet$ & $\bullet$ & $\bullet$ & $\bullet$ & $\bullet$ & $\bullet$ & $\bullet$ & $\bullet$ & $\bullet$ & $\bullet$ & $\bullet$ & $\bullet$ & $\bullet$ & $\bullet$ & $\bullet$ & $\bullet$ & $\bullet$ & $\bullet$ & $\bullet$ & $\bullet$ \\
\hline 04060500 & & & & & & & & & & & & & & & & & & & & & & & $\bullet$ & $\bullet$ & $\bullet$ & $\bullet$ & $\bullet$ & $\bullet$ & $\bullet$ & $\bullet$ \\
\hline 04060993 & 0 & 0 & 0 & $\circ$ & 0 & 0 & $\circ$ & $\circ$ & 0 & 0 & 0 & O & 0 & 0 & $\circ$ & 0 & 0 & 0 & 0 & 0 & 0 & 0 & $\circ$ & O & $\circ$ & $\circ$ & $\circ$ & $\circ$ & 0 & o \\
\hline 04061000 & $\bullet$ & - & - & $\bullet$ & $\bullet$ & $\bullet$ & $\bullet$ & - & $\bullet$ & $\bullet$ & $\bullet$ & & & & & & & & & & & & & & & & & & & \\
\hline 04061500 & 0 & O & 0 & 0 & 0 & 0 & 0 & 0 & 0 & 0 & $\circ$ & $\circ$ & 0 & 0 & & & & & & & & & & & & & & & & \\
\hline 04062000 & o & 0 & 0 & 0 & 0 & 0 & 0 & 0 & 0 & 0 & 0 & 0 & 0 & 0 & 0 & 0 & 0 & 0 & 0 & 0 & 0 & 0 & 0 & 0 & 0 & 0 & 0 & 0 & 0 & 0 \\
\hline 04062011 & & & & & & & & 0 & 0 & 0 & 0 & 0 & 0 & 0 & $\circ$ & 0 & $\circ$ & 0 & 0 & 0 & 0 & $\circ$ & 0 & $\circ$ & 0 & $\circ$ & $\circ$ & 0 & 0 & 0 \\
\hline 04062100 & & & & & & & & & & & & $\bullet$ & - & & & & & & & & & & & & & & & & & \\
\hline 04062200 & & & & & & & & & & & & & & & & & & & $\bullet$ & $\bullet$ & $\bullet$ & $\bullet$ & $\bullet$ & $\bullet$ & $\bullet$ & $\bullet$ & $\bullet$ & $\bullet$ & $\bullet$ & \\
\hline 04062230 & & & & & & & & & & & & & & & & & & & & & & & & & & & & & & \\
\hline 04062270 & & & & & & & & & & & & & & & & & & & & & & & & & & & & & & \\
\hline 04062300 & & & & & & & & & & & & & & & & & & & & & & & & & & & & & & \\
\hline 04062400 & & & & & & & & & & & & & & & & & & & & & & & & & & & & & & \\
\hline 04062500 & 0 & 0 & 0 & 0 & 0 & 0 & 0 & 0 & 0 & 0 & 0 & 0 & 0 & 0 & 0 & 0 & 0 & 0 & 0 & 0 & 0 & 0 & 0 & 0 & 0 & 0 & 0 & 0 & 0 & 0 \\
\hline 04063000 & 0 & $\circ$ & 0 & 0 & 0 & 0 & 0 & 0 & 0 & 0 & 0 & $\circ$ & $\circ$ & 0 & $\circ$ & 0 & 0 & 0 & 0 & 0 & 0 & 0 & 0 & 0 & 0 & 0 & 0 & 0 & 0 & 0 \\
\hline 04063500 & 0 & 0 & 0 & 0 & 0 & 0 & 0 & & 0 & 0 & 0 & 0 & 0 & 0 & 0 & 0 & 0 & 0 & 0 & 0 & $\circ$ & 0 & 0 & 0 & 0 & 0 & 0 & 0 & 0 & 0 \\
\hline 04063640 & & & & & & & & & & & & & & & & & & & & & & & & & & & & & & \\
\hline 04063700 & $\bullet$ & $\bullet$ & - & $\bullet$ & $\bullet$ & $\bullet$ & $\bullet$ & $\bullet$ & $\bullet$ & $\bullet$ & $\bullet$ & $\bullet$ & • & $\bullet$ & $\bullet$ & $\bullet$ & $\bullet$ & - & - & $\bullet$ & $\bullet$ & $\bullet$ & $\bullet$ & - & $\bullet$ & $\bullet$ & $\bullet$ & $\bullet$ & $\bullet$ & $\bullet$ \\
\hline 04064500 & & & & & & & & & & & & & & & $\bullet$ & $\bullet$ & $\bullet$ & - & $\bullet$ & $\bullet$ & $\bullet$ & $\bullet$ & $\bullet$ & $\bullet$ & $\bullet$ & $\bullet$ & $\bullet$ & $\bullet$ & $\bullet$ & $\bullet$ \\
\hline 04065106 & & & & & & & & & & & $\circ$ & 0 & $\circ$ & 0 & 0 & 0 & 0 & 0 & 0 & $\circ$ & 0 & $\circ$ & 0 & 0 & 0 & 0 & $\circ$ & 0 & 0 & 0 \\
\hline
\end{tabular}


Table 5. Streamgaging stations considered for analysis of study area 2 (hydrologic subregion 0403 and cataloguing units 04040002,04040003 , 04060106, 04060107, 04070001, and 04070002) in the U.S. Great Lakes Basin, by water year._Continued

[ $\bullet$, station active and included in the analysis; $\circ$, station active but not included in the analysis]

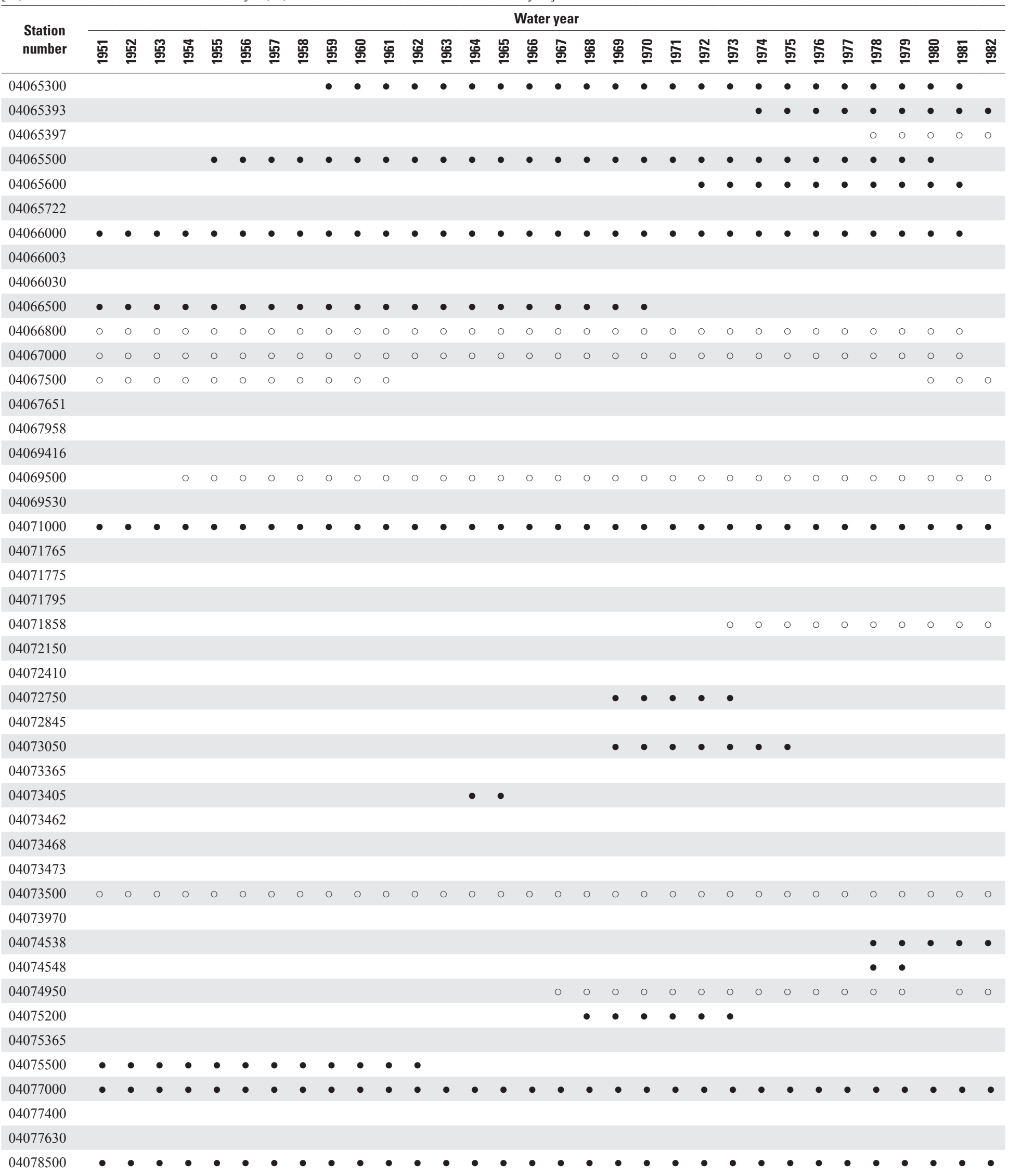


Table 5. Streamgaging stations considered for analysis of study area 2 (hydrologic subregion 0403 and cataloguing units 04040002 , 04040003, 04060106, 04060107, 04070001, and 04070002) in the U.S. Great Lakes Basin, by water year.-Continued

$[\bullet$, station active and included in the analysis; $\circ$, station active but not included in the analysis]

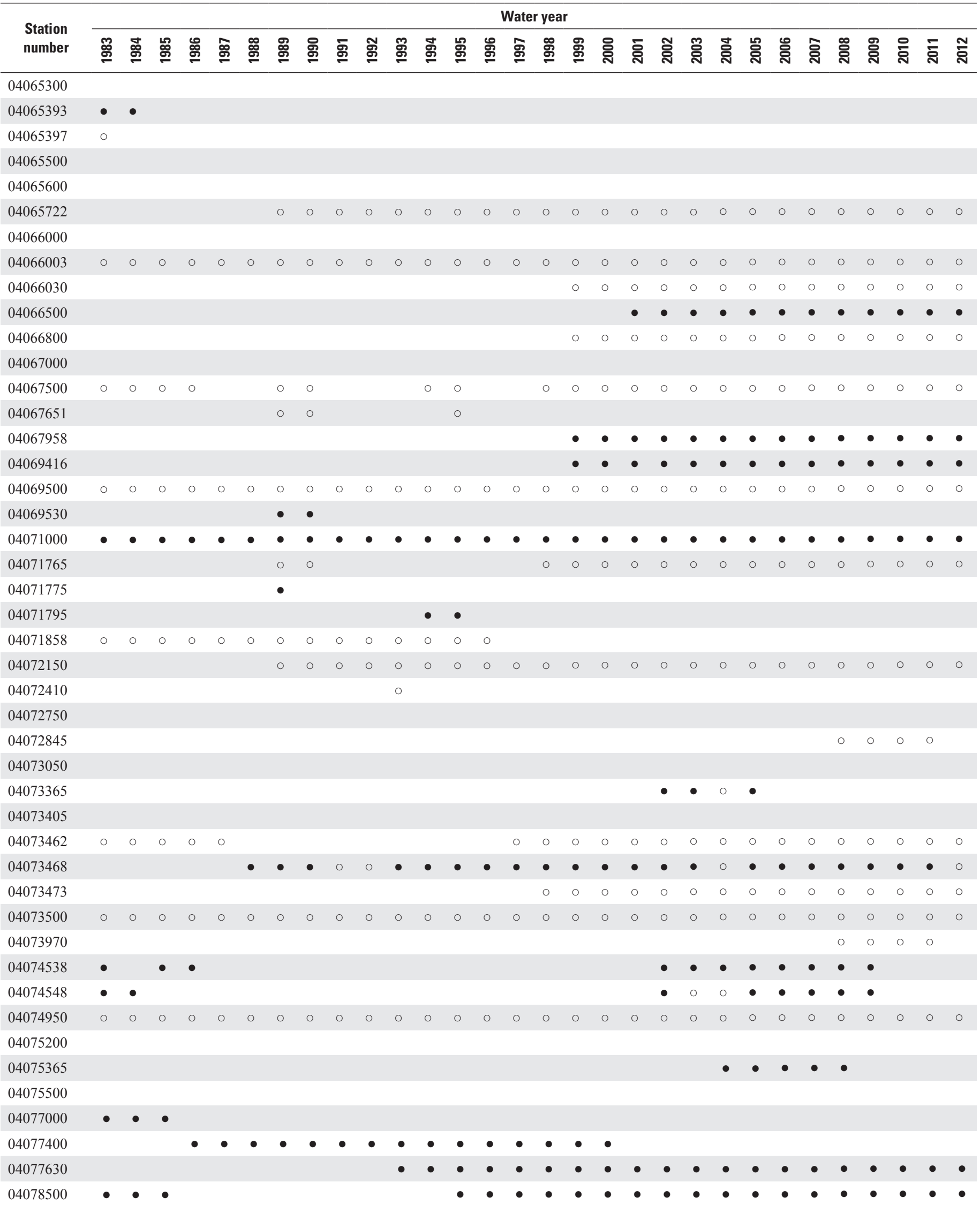


Table 5. Streamgaging stations considered for analysis of study area 2 (hydrologic subregion 0403 and cataloguing units 04040002,04040003 , 04060106, 04060107, 04070001, and 04070002) in the U.S. Great Lakes Basin, by water year.-Continued

$[\bullet$, station active and included in the analysis; $\circ$, station active but not included in the analysis]

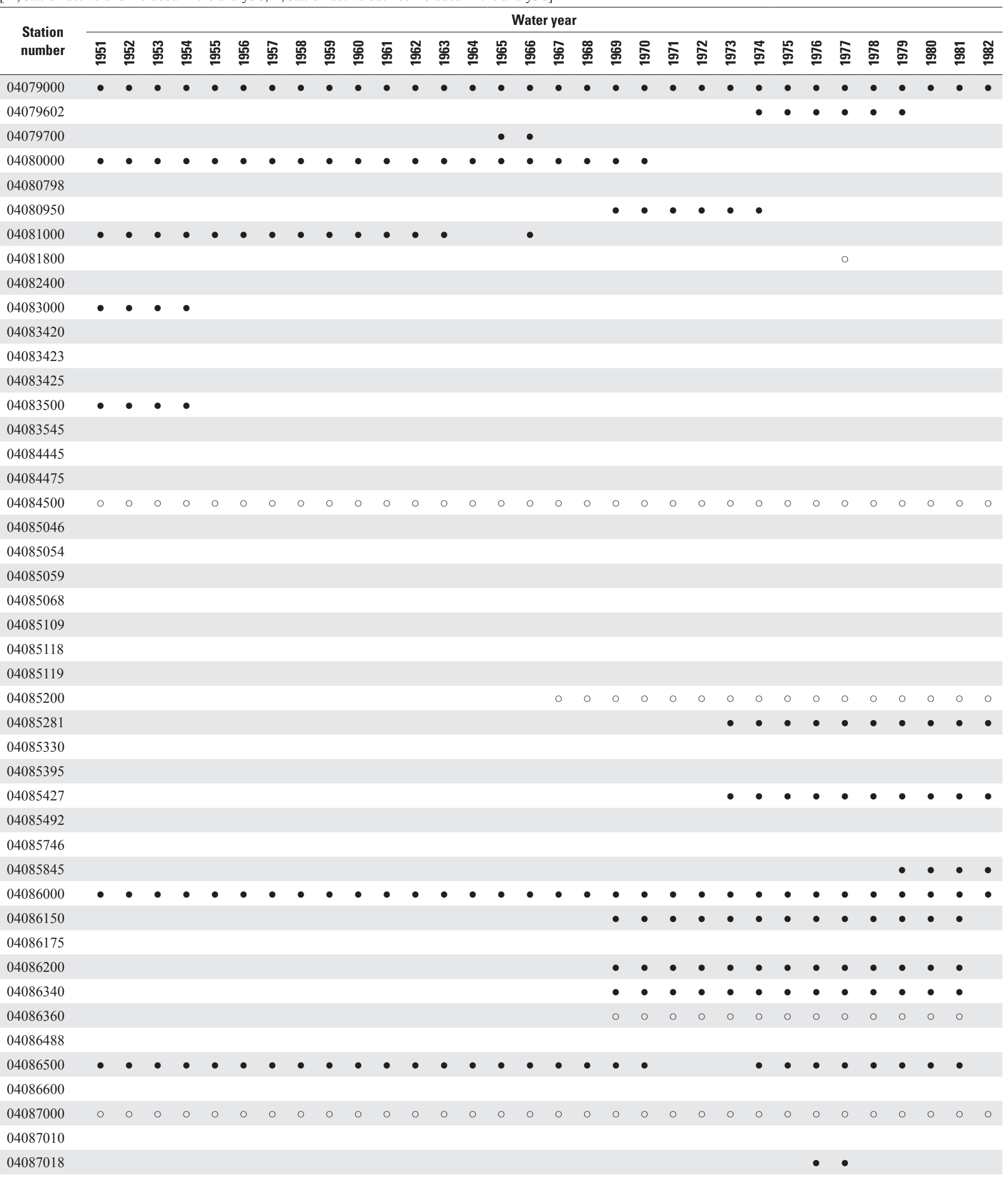


Table 5. Streamgaging stations considered for analysis of study area 2 (hydrologic subregion 0403 and cataloguing units 04040002 , 04040003, 04060106, 04060107, 04070001, and 04070002) in the U.S. Great Lakes Basin, by water year.-Continued

[ $\bullet$, station active and included in the analysis; $\circ$, station active but not included in the analysis]

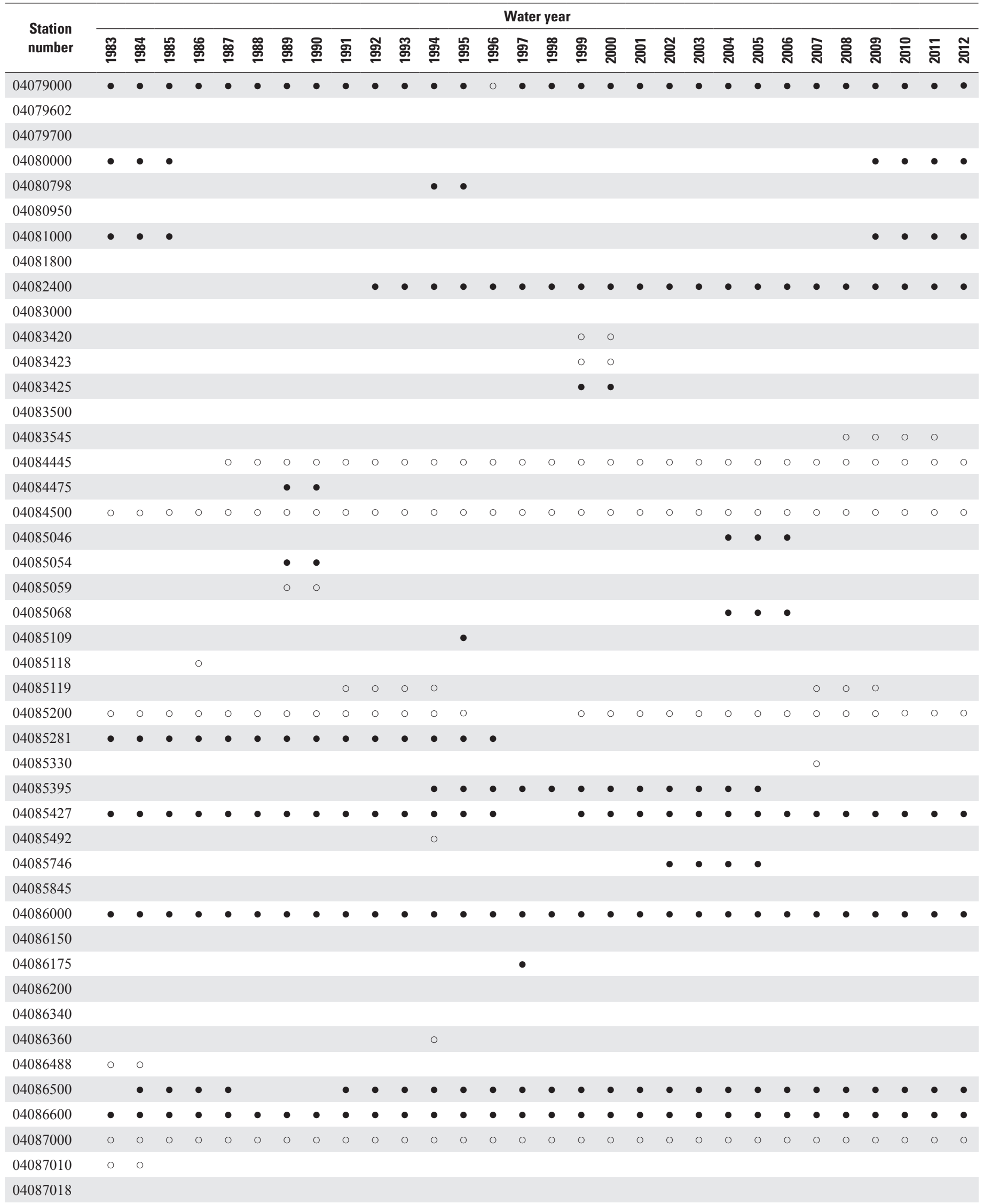


Table 5. Streamgaging stations considered for analysis of study area 2 (hydrologic subregion 0403 and cataloguing units 04040002,04040003 , 04060106, 04060107, 04070001, and 04070002) in the U.S. Great Lakes Basin, by water year.-Continued

[ $\bullet$, station active and included in the analysis; ○, station active but not included in the analysis]

\begin{tabular}{|c|c|c|c|c|c|c|c|c|c|c|c|c|c|c|c|c|c|c|c|c|c|c|c|c|c|c|c|c|c|c|c|c|c|}
\hline \multirow{2}{*}{$\begin{array}{l}\text { Station } \\
\text { number }\end{array}$} & \multicolumn{33}{|c|}{ Water year } \\
\hline & 몸 & ్ํㅁ & ๓్ุ & 范 & 농 & 듀 & 동 & 욤 & 윰 & ஜ & $\bar{\sigma}$ & ๙ั & ஜூ & ஜ్ & 뭉 & ஜே & कृ & ஜ̊ & 맘 & 욤 & б & $\underset{\mathfrak{N}}{\text { న }}$ & $\stackrel{\Re}{\frac{\pi}{\pi}}$ & $\frac{\pi}{\sigma}$ & $\frac{\text { م }}{\sigma}$ & $\underset{\sigma}{\stackrel{0}{\sigma}}$ & ह & $\stackrel{\infty}{\frac{\infty}{\sigma}}$ & $\frac{\sigma}{\sigma}$ & 욤 & : & & \% \\
\hline 04087019 & & & & & & & & & & & & & & & & & & & & & & & & & & & o & o & & & & & \\
\hline 04087030 & & & & & & & & & & & & & & & & & & & & & & & & & & $\bullet$ & $\bullet$ & & & $\bullet$ & 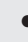 & & $\bullet$ \\
\hline 04087040 & & & & & & & & & & & & & & & & & & & & & & & & & & $\bullet$ & $\bullet$ & $\bullet$ & $\bullet$ & & & & \\
\hline \multicolumn{34}{|l|}{04087047} \\
\hline \multicolumn{34}{|l|}{04087048} \\
\hline 04087050 & & & & & & & & & & & & & & & & & & & & & & & & & & $\bullet$ & $\bullet$ & $\bullet$ & $\bullet$ & & & & \\
\hline 04087060 & & & & & & & & & & & & & & & & & & & & & & & & & & $\bullet$ & $\bullet$ & $\bullet$ & $\bullet$ & & & & \\
\hline 04087070 & & & & & & & & & & & & & & & & & & & & & & & & & & $\bullet$ & o & & & & & & \\
\hline 04087088 & & & & & & & & & & & & & & & & & & & & & & & & & & $\bullet$ & $\bullet$ & $\bullet$ & $\bullet$ & & 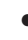 & & $\bullet$ \\
\hline 04087119 & & & & & & & & & & & & & & & & & & & & & & & & & & $\bullet$ & $\bullet$ & $\bullet$ & $\bullet$ & & & & \\
\hline 04087120 & & & & & & & & & & & & $\bullet$ & $\bullet$ & $\bullet$ & $\bullet$ & $\bullet$ & $\bullet$ & $\bullet$ & $\bullet$ & $\bullet$ & $\bullet$ & $\bullet$ & $\bullet$ & $\bullet$ & $\bullet$ & ० & ० & $\bullet$ & $\bullet$ & $\bullet$ & 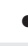 & & $\bullet$ \\
\hline 04087125 & & & & & & & & & & & & & & & & & & & & & & & & & & o & o & ० & o & & & & \\
\hline \multicolumn{34}{|l|}{04087138} \\
\hline 04087140 & & & & & & & & & & & & & & & & & & & & & & & & & & o & & & & & & & \\
\hline \multicolumn{34}{|l|}{04087142} \\
\hline \multicolumn{34}{|l|}{04087159} \\
\hline 04087160 & & & & & & & & & & & & & & & & & & & & & & & & & & & 0 & 0 & o & $\circ$ & $c$ & & $\circ$ \\
\hline \multicolumn{34}{|l|}{04087170} \\
\hline 04087204 & & & & & & & & & & & & & & $\bullet$ & $\bullet$ & $\bullet$ & $\bullet$ & $\bullet$ & $\bullet$ & $\bullet$ & $\bullet$ & $\bullet$ & $\bullet$ & $\bullet$ & $\bullet$ & $\bullet$ & $\bullet$ & $\bullet$ & $\bullet$ & $\bullet$ & 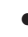 & & $\bullet$ \\
\hline \multicolumn{34}{|l|}{04087214} \\
\hline 04087220 & & & & & & & & & & & & & & $\bullet$ & $\bullet$ & $\bullet$ & $\bullet$ & $\bullet$ & $\bullet$ & $\bullet$ & $\bullet$ & $\bullet$ & $\bullet$ & $\bullet$ & $\bullet$ & $\bullet$ & $\bullet$ & $\bullet$ & $\bullet$ & $\bullet$ & 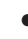 & & $\bullet$ \\
\hline 04087233 & & & & & & & & & & & & & & $\bullet$ & $\bullet$ & $\bullet$ & $\bullet$ & $\bullet$ & $\bullet$ & $\bullet$ & $\bullet$ & $\bullet$ & $\bullet$ & $\bullet$ & - & $\bullet$ & $\bullet$ & $\bullet$ & $\bullet$ & $\bullet$ & 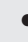 & & $\bullet$ \\
\hline 04087240 & & & & & & & & & & & & & & $\circ$ & $\circ$ & $\circ$ & ० & ० & $\circ$ & $\circ$ & $\circ$ & $\circ$ & $\circ$ & $\circ$ & ० & ० & 0 & ० & ○ & $\circ$ & $c$ & & $\circ$ \\
\hline 04087257 & & & & & & & & & & & & & & & & & & & & & & $\bullet$ & $\bullet$ & $\bullet$ & $\bullet$ & - & $\bullet$ & $\bullet$ & $\bullet$ & $\bullet$ & 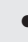 & & $\bullet$ \\
\hline 04127918 & & & & & & & & & & & & & & & & & & & & & & & $\bullet$ & $\bullet$ & $\bullet$ & $\bullet$ & $\bullet$ & $\bullet$ & $\bullet$ & $\bullet$ & 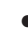 & & $\bullet$ \\
\hline \multicolumn{34}{|l|}{040445315} \\
\hline 040590345 & & & & & & & & & & & & & & & & & & & & & & & & & & & & & & & & & \\
\hline 040734644 & & & & & & & & & & & & & & & & & & & & & & & & & & & & & & & & & \\
\hline 040851325 & & & & & & & & & & & & & & & & & & & & & & & & & & & & & & & & & \\
\hline 040851378 & & & & & & & & & & & & & & & & & & & & & & & & & & & & & & & & & \\
\hline 040851385 & & & & & & & & & & & & & & & & & & & & & & & & & & & & & & & & & \\
\hline 040857005 & & & & & & & & & & & & & & & & & & & & & & & & & & & & & & & & & \\
\hline 040860041 & & & & & & & & & & & & & & & & & & & & & & & & & & & & & & & & & \\
\hline 040863075 & & & & & & & & & & & & & & & & & & & & & & & & & & & & & & & & & \\
\hline 040869415 & & & & & & & & & & & & & & & & & & & & & & & & & & & & & & & & & \\
\hline 040869416 & & & & & & & & & & & & & & & & & & & & & & & & & & & & & & & & & \\
\hline 040871473 & & & & & & & & & & & & & & & & & & & & & & & & & & & & & & & & & \\
\hline 040871475 & & & & & & & & & & & & & & & & & & & & & & & & & & & & & & & & & \\
\hline 040871476 & & & & & & & & & & & & & & & & & & & & & & & & & & & & & & & & & \\
\hline 040871488 & & & & & & & & & & & & & & & & & & & & & & & & & & & & & & & & & \\
\hline 040874126 & & & & & & & & & & & & & & & & & & & & & & & & & & & & & & & & & \\
\hline 0407809265 & & & & & & & & & & & & & & & & & & & & & & & & & & & & & & & & & \\
\hline 0408570045 & & & & & & & & & & & & & & & & & & & & & & & & & & & & & & & & & \\
\hline
\end{tabular}


Table 5. Streamgaging stations considered for analysis of study area 2 (hydrologic subregion 0403 and cataloguing units 04040002 , 04040003, 04060106, 04060107, 04070001, and 04070002) in the U.S. Great Lakes Basin, by water year.-Continued

[ $\bullet$, station active and included in the analysis; ○, station active but not included in the analysis]

\begin{tabular}{|c|c|c|c|c|c|c|c|c|c|c|c|c|c|c|c|c|c|c|c|c|c|c|c|c|c|c|c|c|c|c|}
\hline \multirow{2}{*}{$\begin{array}{l}\text { Station } \\
\text { number }\end{array}$} & \multicolumn{30}{|c|}{ Water year } \\
\hline & ஜூ: & 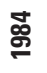 & 요 & ஜீ & ळ & 吕 & 吕 & 윰 & Б্ & శ్ & ஜ뮤 & 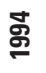 & 농 & ஜூ & જூ & 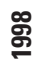 & 욤 & 욤 & ธ్ర & ণั & ஜ̊̊̆ & ণั & ํㅗㅇ & 乌̊ & ్ㅗำ & 号 & 용 & 음 & 모 & ํํํ \\
\hline \multicolumn{31}{|l|}{04087019} \\
\hline 04087030 & $\bullet$ & $\bullet$ & $\bullet$ & $\bullet$ & $\bullet$ & $\bullet$ & $\bullet$ & $\bullet$ & $\bullet$ & $\bullet$ & $\bullet$ & $\bullet$ & $\bullet$ & $\bullet$ & $\bullet$ & $\bullet$ & $\bullet$ & $\bullet$ & $\bullet$ & $\bullet$ & $\bullet$ & $\bullet$ & $\bullet$ & $\bullet$ & $\bullet$ & $\bullet$ & $\bullet$ & $\bullet$ & $\bullet$ & $\bullet$ \\
\hline \multicolumn{31}{|l|}{04087040} \\
\hline 04087047 & & & & & & & & & & & & & & & & & & & & & & & & & & 0 & & & & \\
\hline 04087048 & & & & & & & & & & & & & & & & & & & & & & & & & & ० & & & & \\
\hline 04087050 & & & & & & & & & & & & & & & & & & & & & & & & & & $\bullet$ & $\bullet$ & $\bullet$ & $\bullet$ & $\bullet$ \\
\hline \multicolumn{31}{|l|}{04087060} \\
\hline 04087070 & & & & & & & & & & & & & & & & & & & & & & & $\bullet$ & $\bullet$ & $\bullet$ & $\bullet$ & $\bullet$ & $\bullet$ & $\bullet$ & $\bullet$ \\
\hline 04087088 & $\bullet$ & $\bullet$ & $\bullet$ & $\bullet$ & $\bullet$ & $\bullet$ & $\bullet$ & $\bullet$ & $\bullet$ & $\bullet$ & $\bullet$ & $\bullet$ & $\bullet$ & $\bullet$ & $\bullet$ & $\bullet$ & $\bullet$ & $\bullet$ & $\bullet$ & $\bullet$ & $\bullet$ & $\bullet$ & $\bullet$ & $\bullet$ & $\bullet$ & $\bullet$ & $\bullet$ & $\bullet$ & $\bullet$ & $\bullet$ \\
\hline 04087119 & & & & & & & & & & & & & & & & & & & & & & & $\bullet$ & $\bullet$ & $\bullet$ & $\bullet$ & $\bullet$ & $\bullet$ & $\bullet$ & $\bullet$ \\
\hline 04087120 & $\bullet$ & $\bullet$ & $\bullet$ & $\bullet$ & $\bullet$ & $\bullet$ & $\bullet$ & $\bullet$ & $\bullet$ & $\bullet$ & $\bullet$ & $\bullet$ & $\bullet$ & $\bullet$ & $\bullet$ & $\bullet$ & $\bullet$ & $\bullet$ & $\bullet$ & $\bullet$ & $\bullet$ & $\bullet$ & $\bullet$ & $\bullet$ & $\bullet$ & $\bullet$ & $\bullet$ & $\bullet$ & $\bullet$ & ○ \\
\hline \multicolumn{31}{|l|}{04087125} \\
\hline 04087138 & $\circ$ & $\circ$ & & & & & & & & & & & & & & & & & & & & & & & & & & & & \\
\hline \multicolumn{31}{|l|}{04087140} \\
\hline 04087142 & & & & & & & & & & & & & & & & & & & & & & & & & & & o & ० & o & 0 \\
\hline 04087159 & $\bullet$ & - & $\bullet$ & - & - & - & - & $\bullet$ & $\bullet$ & $\bullet$ & $\bullet$ & - & $\bullet$ & - & • & - & - & - & $\bullet$ & $\bullet$ & $\bullet$ & $\bullet$ & $\bullet$ & $\bullet$ & • & $\bullet$ & $\bullet$ & $\bullet$ & $\bullet$ & $\bullet$ \\
\hline \multicolumn{31}{|l|}{04087160} \\
\hline 04087170 & & & & & & & & & & & & & $\bullet$ & & & & & & & $\bullet$ & $\bullet$ & & & & $\bullet$ & $\bullet$ & $\bullet$ & $\bullet$ & $\bullet$ & $\bullet$ \\
\hline 04087204 & $\bullet$ & $\bullet$ & $\bullet$ & $\bullet$ & $\bullet$ & $\bullet$ & $\bullet$ & $\bullet$ & $\bullet$ & $\bullet$ & $\bullet$ & $\bullet$ & $\bullet$ & $\bullet$ & $\bullet$ & - & $\bullet$ & $\bullet$ & $\bullet$ & $\bullet$ & $\bullet$ & $\bullet$ & $\bullet$ & $\bullet$ & $\bullet$ & $\bullet$ & $\bullet$ & $\bullet$ & $\bullet$ & $\bullet$ \\
\hline 04087214 & & & & & & & & & & & & & & & & & & & & & & & $\circ$ & $\circ$ & ० & ० & o & ○ & o & ० \\
\hline 04087220 & $\bullet$ & • & $\bullet$ & $\bullet$ & $\bullet$ & $\bullet$ & $\bullet$ & $\bullet$ & $\bullet$ & $\bullet$ & $\bullet$ & $\bullet$ & $\bullet$ & $\bullet$ & $\bullet$ & $\bullet$ & $\bullet$ & $\bullet$ & $\bullet$ & $\bullet$ & $\bullet$ & $\bullet$ & $\bullet$ & $\bullet$ & $\bullet$ & $\bullet$ & $\bullet$ & $\bullet$ & $\bullet$ & $\bullet$ \\
\hline 04087233 & $\bullet$ & $\bullet$ & $\bullet$ & - & $\bullet$ & $\bullet$ & $\bullet$ & $\bullet$ & $\bullet$ & $\bullet$ & $\bullet$ & $\bullet$ & $\bullet$ & $\bullet$ & $\bullet$ & - & $\bullet$ & $\bullet$ & $\bullet$ & $\bullet$ & $\bullet$ & $\bullet$ & $\bullet$ & $\bullet$ & • & $\bullet$ & • & $\bullet$ & - & $\bullet$ \\
\hline 04087240 & o & ० & ० & o & ० & 0 & o & ○ & O & $\circ$ & $\circ$ & ० & $\circ$ & 0 & ० & o & o & ० & $\circ$ & $\circ$ & O & $\circ$ & 0 & ○ & ० & ० & O & O & ० & ० \\
\hline 04087257 & $\bullet$ & $\bullet$ & $\bullet$ & $\bullet$ & $\bullet$ & $\bullet$ & $\bullet$ & $\bullet$ & $\bullet$ & $\bullet$ & $\bullet$ & $\bullet$ & $\bullet$ & $\bullet$ & $\bullet$ & $\bullet$ & $\bullet$ & $\bullet$ & $\bullet$ & $\bullet$ & $\bullet$ & $\bullet$ & $\bullet$ & $\bullet$ & $\bullet$ & $\bullet$ & $\bullet$ & $\bullet$ & $\bullet$ & $\bullet$ \\
\hline 04127918 & $\bullet$ & $\bullet$ & $\bullet$ & $\bullet$ & $\bullet$ & $\bullet$ & $\bullet$ & $\bullet$ & $\bullet$ & $\bullet$ & $\bullet$ & $\bullet$ & $\bullet$ & $\bullet$ & $\bullet$ & $\bullet$ & $\bullet$ & $\bullet$ & $\bullet$ & $\bullet$ & $\bullet$ & $\bullet$ & $\bullet$ & $\bullet$ & $\bullet$ & $\bullet$ & $\bullet$ & $\bullet$ & $\bullet$ & ० \\
\hline 040445315 & & & & & & & & & & & & & & & & o & o & & & & & & & & & & & & & \\
\hline 040590345 & & & & & & & o & $\circ$ & & & & & & & & & & & & & & & & & & & & & & \\
\hline 040734644 & & & & & & $\bullet$ & $\bullet$ & $\bullet$ & $\bullet$ & $\bullet$ & $\bullet$ & $\bullet$ & $\bullet$ & $\bullet$ & & & & & & & & & & & & & & & & \\
\hline 040851325 & & & & & & & & & & & & & & & & & & & & & & $\bullet$ & $\bullet$ & $\bullet$ & $\bullet$ & & & & & \\
\hline 040851378 & & & & & & & & & & & & & & & & & & & & & & & $\bullet$ & $\bullet$ & $\bullet$ & & & & & \\
\hline 040851385 & & & & & & & ० & $\circ$ & $\circ$ & $\circ$ & $\circ$ & ० & $\circ$ & $\circ$ & ० & $\circ$ & o & $\circ$ & $\circ$ & $\circ$ & ○ & $\circ$ & $\circ$ & ○ & ० & ० & ○ & ○ & ० & ० \\
\hline 040857005 & & & & & & & & & $\bullet$ & $\bullet$ & $\bullet$ & $\bullet$ & $\bullet$ & $\bullet$ & $\bullet$ & $\bullet$ & $\bullet$ & $\bullet$ & $\bullet$ & $\bullet$ & & & & & & & & & & \\
\hline 040860041 & & & & & & & & & & & & ० & $\circ$ & & & & & & & & & & & & & & & & & \\
\hline 040863075 & & & & & & & & & & & & $\bullet$ & $\bullet$ & & & & & & & & & & & & & & & & & \\
\hline 040869415 & & & & & & & & & & & & ० & & & & & & & & & & & & & & & & & & \\
\hline 040869416 & & & & & & & & & & & & & & & & & & & & & & $\bullet$ & $\bullet$ & $\bullet$ & $\bullet$ & $\bullet$ & $\bullet$ & $\bullet$ & $\bullet$ & $\bullet$ \\
\hline 040871473 & & & & & & & & & & & & & & & & & O & $\circ$ & $\circ$ & ० & $\circ$ & $\circ$ & O & ० & ० & ० & ० & ○ & ० & O \\
\hline 040871475 & & & & & & & & & & & & & & & & & & & & & & & & ० & ० & ० & ० & ○ & ० & ० \\
\hline 040871476 & & & & & & & & & & & & & & & & & o & $\circ$ & $\circ$ & $\circ$ & $\circ$ & $\circ$ & $\circ$ & ० & $\circ$ & $\circ$ & ० & ○ & ○ & ० \\
\hline 040871488 & & & & & & & & & & & & & & & & & o & 0 & ० & ० & ○ & $\circ$ & ० & ० & ० & o & ० & ० & ० & o \\
\hline 040874126 & & & & & & & & & & & & & & $\circ$ & & & & & & & & & & & & & & & & \\
\hline 0407809265 & & & & & & & & $\bullet$ & $\bullet$ & $\bullet$ & $\bullet$ & $\bullet$ & $\bullet$ & $\bullet$ & $\bullet$ & $\bullet$ & $\bullet$ & $\bullet$ & $\bullet$ & $\bullet$ & $\bullet$ & $\bullet$ & $\bullet$ & $\bullet$ & & & & & & \\
\hline 0408570045 & & & & & & & & & & & & & $\circ$ & $\circ$ & & & & & & & & & & & & & & & & \\
\hline
\end{tabular}


Table 6. Regression statistics and explanatory variables used in the AFINCH (Analysis of Flows In Networks of Channels) analysis for study area 2 (hydrologic subregion 0403 and cataloguing units 04040002, 04040003, 04060106, 04060107, 04070001, and 04070002) in the U.S. Great Lakes Basin, by month.

[-, indicates variable not included in monthly regression equation; RMSE, root mean squared error; $\mathrm{R}^{2}$, coefficient of determination. See appendix tables for variable descriptions]

\begin{tabular}{|c|c|c|c|c|c|c|c|c|c|c|c|c|c|c|}
\hline \multirow[b]{2}{*}{ Month } & \multicolumn{11}{|c|}{ Regression coefficients } & \multicolumn{3}{|c|}{ Regression fit statistics } \\
\hline & Intercept & $\begin{array}{c}\text { Current } \\
\text { precipi- } \\
\text { tation }\end{array}$ & $\begin{array}{l}\text { Preceding } \\
\text { precipita- } \\
\text { tion }\end{array}$ & $\begin{array}{c}\text { Current } \\
\text { tempera- } \\
\text { ture }\end{array}$ & $\begin{array}{l}\text { Pasture } \\
\text { hay and } \\
\text { row crops }\end{array}$ & Forest & $\begin{array}{c}\text { Wetland } \\
\text { woody }\end{array}$ & $\begin{array}{c}\text { Wetland } \\
\text { herbaceous }\end{array}$ & $\begin{array}{l}\text { Hydric } \\
\text { group C } \\
\text { and CD }\end{array}$ & $\begin{array}{c}\text { Coarse } \\
\text { stratified } \\
\text { sediment }\end{array}$ & Till & RMSE & F-statistic & $\mathbf{R}^{2}$ \\
\hline November & 0.336 & 0.139 & 0.076 & -0.006 & -0.167 & 0.131 & - & - & -0.100 & 0.125 & - & 0.194 & 408.2 & 0.579 \\
\hline January & 0.411 & 0.091 & 0.086 & 0.020 & -0.077 & 0.510 & -0.585 & 0.525 & - & 0.309 & 0.111 & 0.194 & 150.4 & 0.395 \\
\hline March & 0.653 & 0.119 & 0.097 & 0.029 & 0.225 & - & - & 0.839 & 0.293 & - & - & 0.285 & 282.8 & 0.450 \\
\hline May & 1.238 & 0.134 & 0.068 & -0.033 & -0.221 & - & 0.537 & - & - & -0.430 & -0.390 & 0.271 & 345.3 & 0.538 \\
\hline July & 1.057 & 0.103 & 0.066 & -0.028 & -0.518 & -0.232 & -0.800 & - & -0.176 & 0.231 & - & 0.197 & 404.7 & 0.609 \\
\hline September & 0.411 & 0.106 & 0.050 & - & -0.410 & - & -0.600 & -0.341 & -0.189 & 0.159 & - & 0.203 & 446 & 0.601 \\
\hline
\end{tabular}

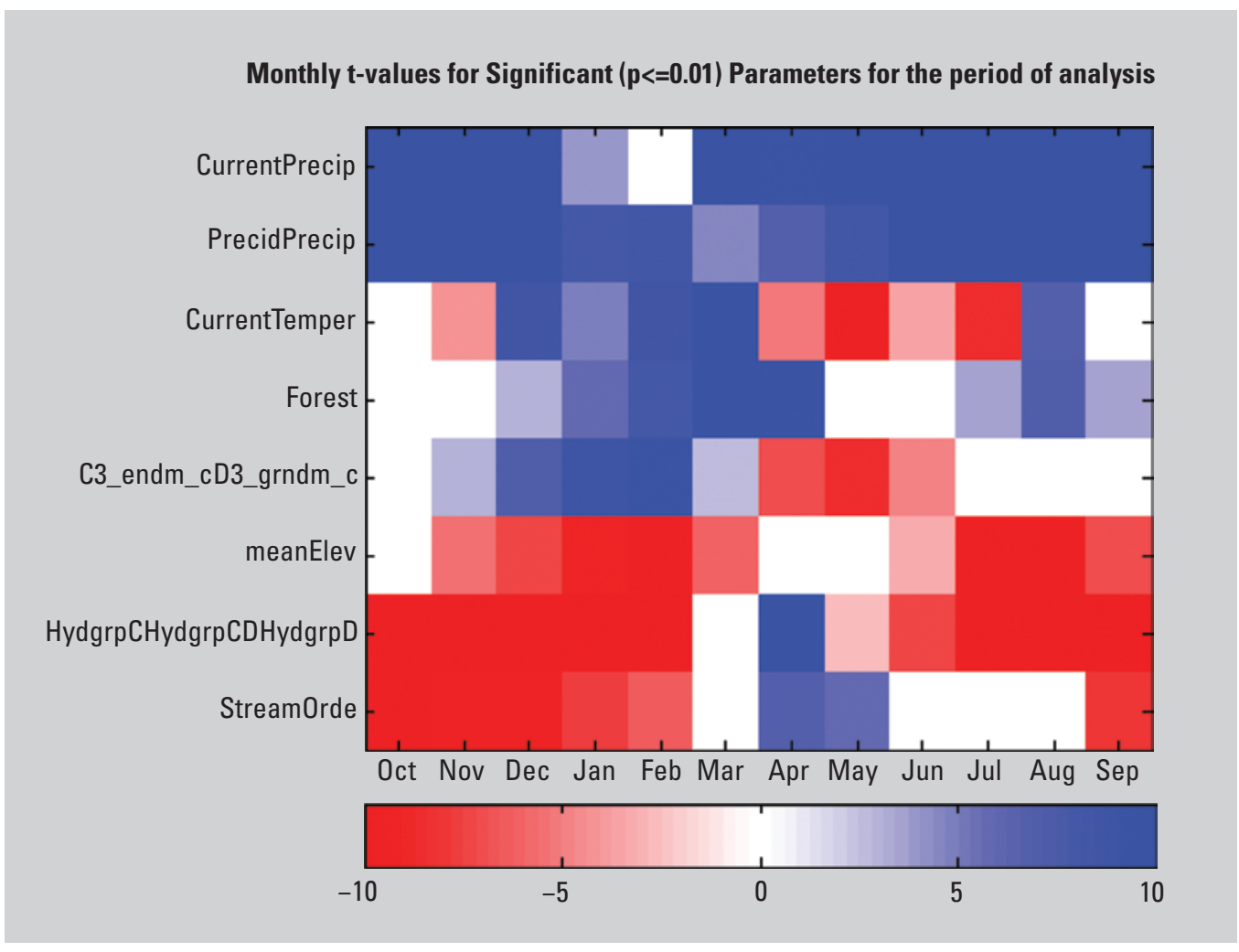

Figure 7. Image showing the t-value-indexed colors corresponding to each explanatory variable in the monthly regression equation for estimating water yield for study area 2 (hydrologic subregion 0403 and cataloguing units 04040002, 04040003, 04060106, 04060107, 04070001, and 04070002) in the U.S. Great Lakes Basin. 


\section{Study Area 3}

Study area 3 is formed by hydrologic subregion 0405 and cataloguing unit 04040001 in the south-central part of the U.S. Great Lakes Basin along the southeastern side of Lake Michigan and encompasses parts of Michigan, Indiana, and a small part of Illinois (fig. 8). The number of stations included in the analysis ranged from 27 (1951) to 54 (1976) (table 7). Thirteen explanatory variables were selected during the regression analysis with the number of variables specified for each monthly equation ranging from 5 (April) to 12 (September) (table 3). Variables and regression statistics based on the stepwise regression for each month are indicated in table 8 and figure 9 .

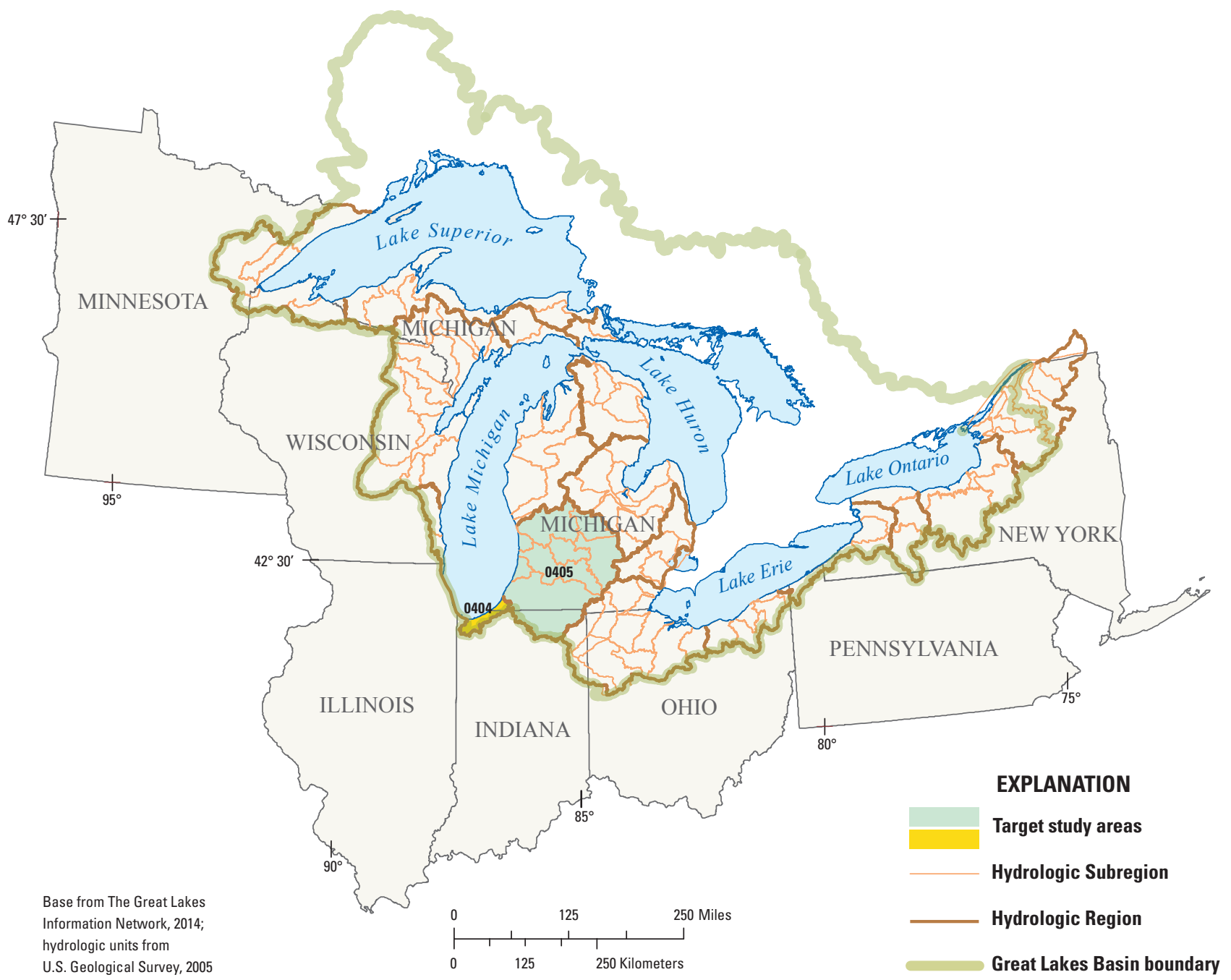

Figure 8. Location of study area 3 (hydrologic subregion 0405 and cataloguing unit 04040001 ) in the U.S. Great Lakes Basin. 
Table 7. Streamgaging stations considered for analysis of study area 3 (hydrologic subregion 0405 and cataloguing unit 04040001 ) in the U.S. Great Lakes Basin, by water year.-Continued

$[\bullet$, station active and included in the analysis; ○, station active but not included in the analysis]

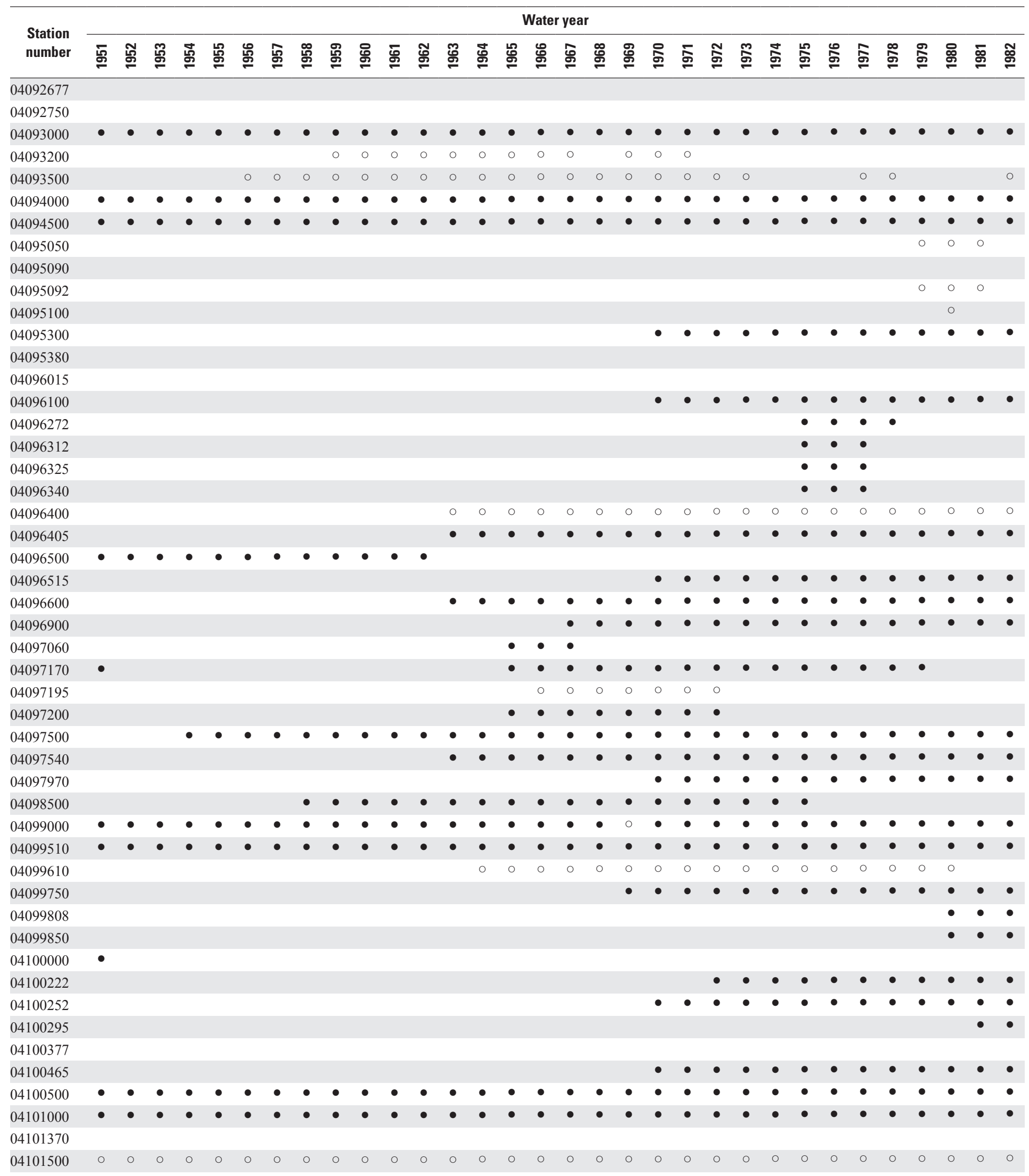


Table 7. Streamgaging stations considered for analysis of study area 3 (hydrologic subregion 0405 and cataloguing unit 04040001 ) in the U.S. Great Lakes Basin, by water year.-Continued

[, station active and included in the analysis; $\bigcirc$, station active but not included in the analysis]

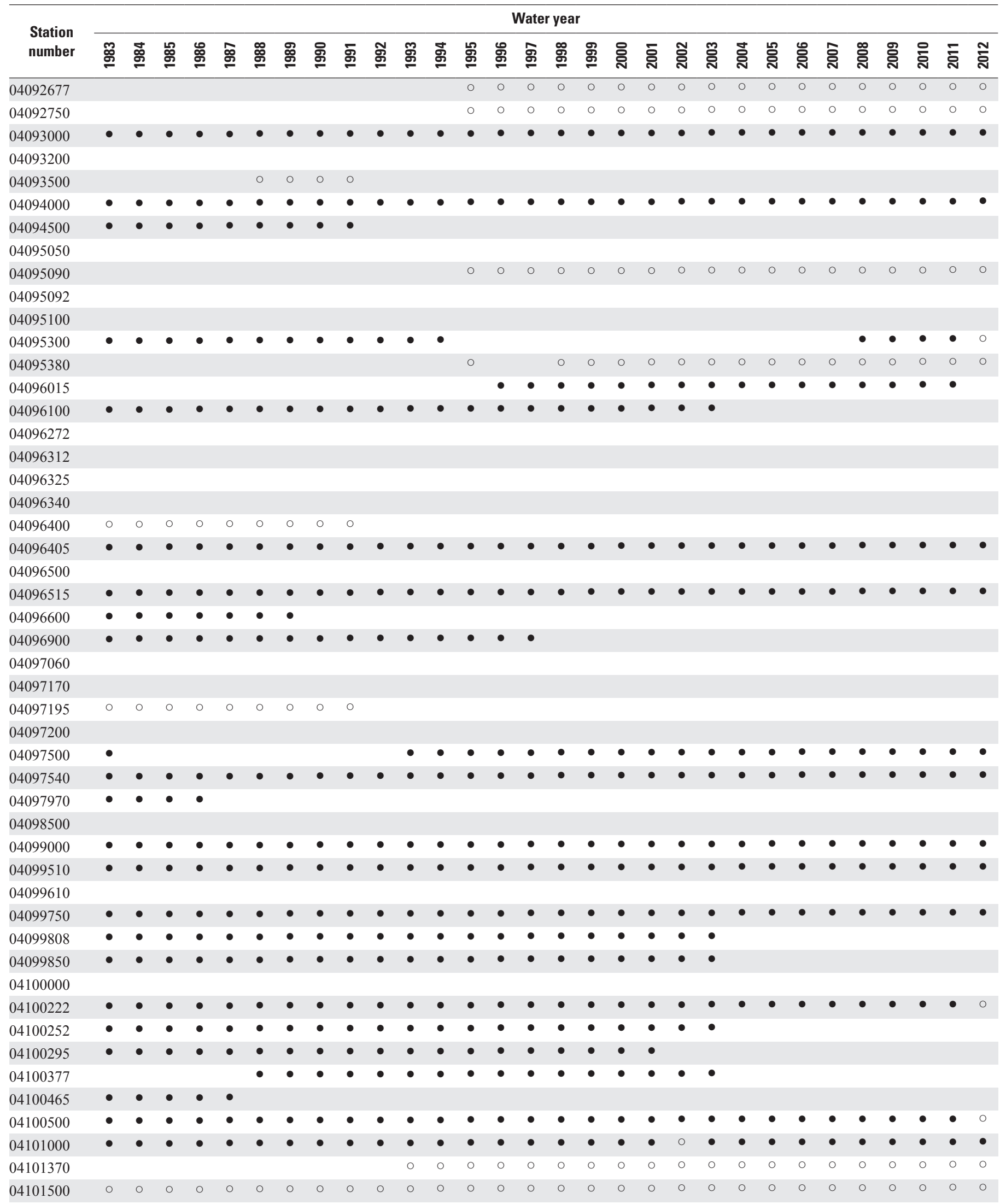


Table 7. Streamgaging stations considered for analysis of study area 3 (hydrologic subregion 0405 and cataloguing unit 04040001 ) in the U.S. Great Lakes Basin, by water year.-Continued

[ $\bullet$, station active and included in the analysis; $\bigcirc$, station active but not included in the analysis]

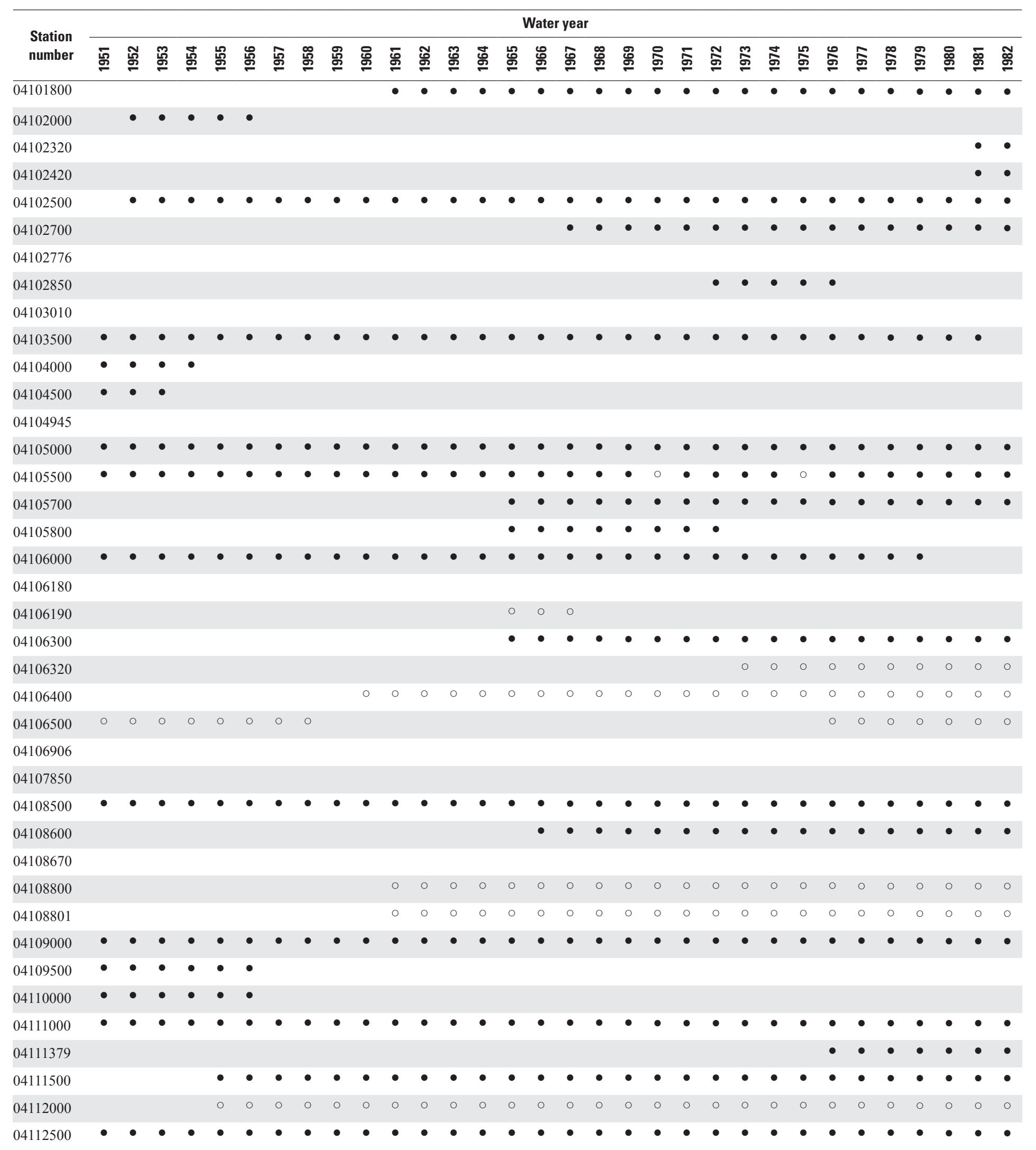


Table 7. Streamgaging stations considered for analysis of study area 3 (hydrologic subregion 0405 and cataloguing unit 04040001 ) in the U.S. Great Lakes Basin, by water year.-Continued

[, station active and included in the analysis; $\bigcirc$, station active but not included in the analysis]

\begin{tabular}{|c|c|c|c|c|c|c|c|c|c|c|c|c|c|c|c|c|c|c|c|c|c|c|c|c|c|c|c|c|c|c|}
\hline \multirow{2}{*}{$\begin{array}{l}\text { Station } \\
\text { number }\end{array}$} & \multicolumn{30}{|c|}{ Water year } \\
\hline & ஜூ & 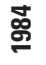 & 筒 & ஜ & 㽞 & 曲 & 吕 & 욤 & হু & ชু & ஜூ & 急 & 늉 & ஜூת & 횽 & ஓ̊ & 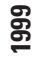 & ్ㅗㅇ & ర్రి & ญ్ & ్ํํ & 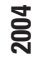 & 농 & 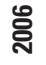 & ్ㅗㅇ & 号 & 융 & 응 & 휴 & ํㅗํ \\
\hline 04101800 & $\bullet$ & $\bullet$ & $\bullet$ & $\bullet$ & $\bullet$ & $\bullet$ & $\bullet$ & $\bullet$ & $\bullet$ & $\bullet$ & $\bullet$ & $\bullet$ & $\bullet$ & $\bullet$ & $\bullet$ & $\bullet$ & $\bullet$ & $\bullet$ & $\bullet$ & $\bullet$ & $\bullet$ & $\bullet$ & $\bullet$ & $\bullet$ & $\bullet$ & $\bullet$ & $\bullet$ & $\bullet$ & $\bullet$ & $\bullet$ \\
\hline \multicolumn{31}{|l|}{04102000} \\
\hline \multicolumn{31}{|l|}{04102320} \\
\hline \multicolumn{31}{|l|}{04102420} \\
\hline 04102500 & $\bullet$ & $\bullet$ & $\bullet$ & $\bullet$ & $\bullet$ & $\bullet$ & $\bullet$ & $\bullet$ & $\bullet$ & $\bullet$ & $\bullet$ & $\bullet$ & $\bullet$ & $\bullet$ & $\bullet$ & $\bullet$ & $\bullet$ & $\bullet$ & $\bullet$ & $\bullet$ & $\bullet$ & $\bullet$ & $\bullet$ & $\bullet$ & $\bullet$ & $\bullet$ & $\bullet$ & $\bullet$ & $\bullet$ & $\bullet$ \\
\hline 04102700 & - & $\bullet$ & $\bullet$ & $\bullet$ & $\bullet$ & $\bullet$ & $\bullet$ & - & $\bullet$ & $\bullet$ & $\bullet$ & $\bullet$ & $\bullet$ & $\bullet$ & - & $\bullet$ & - & $\bullet$ & $\bullet$ & $\bullet$ & $\bullet$ & $\bullet$ & $\bullet$ & $\bullet$ & $\bullet$ & $\bullet$ & $\bullet$ & $\bullet$ & $\bullet$ & $\bullet$ \\
\hline 04102776 & & & & & & & & & & & & & $\bullet$ & $\bullet$ & $\bullet$ & $\bullet$ & - & $\bullet$ & $\bullet$ & $\bullet$ & $\bullet$ & $\bullet$ & $\bullet$ & $\bullet$ & $\bullet$ & $\bullet$ & $\bullet$ & $\bullet$ & $\bullet$ & \\
\hline \multicolumn{31}{|l|}{04102850} \\
\hline 04103010 & & & & & $\bullet$ & $\bullet$ & $\bullet$ & $\bullet$ & $\bullet$ & $\bullet$ & $\bullet$ & $\bullet$ & $\bullet$ & $\bullet$ & $\bullet$ & $\bullet$ & $\bullet$ & $\bullet$ & $\bullet$ & $\bullet$ & $\bullet$ & $\bullet$ & $\bullet$ & $\bullet$ & $\bullet$ & $\bullet$ & $\bullet$ & $\bullet$ & $\bullet$ & \\
\hline 04103500 & & & & & & & & & & & & & & & & & & & & $\bullet$ & $\bullet$ & $\bullet$ & $\bullet$ & $\bullet$ & $\bullet$ & $\bullet$ & $\bullet$ & $\bullet$ & $\bullet$ & $\bullet$ \\
\hline \multicolumn{31}{|l|}{04104000} \\
\hline \multicolumn{31}{|l|}{04104500} \\
\hline 04104945 & & & & & & & & & & & & & $\bullet$ & $\bullet$ & $\bullet$ & $\bullet$ & $\bullet$ & $\bullet$ & $\bullet$ & $\bullet$ & $\bullet$ & $\bullet$ & $\bullet$ & $\bullet$ & $\bullet$ & $\bullet$ & $\bullet$ & $\bullet$ & $\bullet$ & $\bullet$ \\
\hline 04105000 & $\bullet$ & $\bullet$ & $\bullet$ & $\bullet$ & $\bullet$ & $\bullet$ & $\bullet$ & - & $\bullet$ & $\bullet$ & $\bullet$ & $\bullet$ & $\bullet$ & $\bullet$ & $\bullet$ & $\bullet$ & $\bullet$ & $\bullet$ & $\bullet$ & $\bullet$ & $\bullet$ & $\bullet$ & $\bullet$ & $\bullet$ & $\bullet$ & $\bullet$ & $\bullet$ & $\bullet$ & $\bullet$ & $\bullet$ \\
\hline 04105500 & $\bullet$ & $\bullet$ & $\bullet$ & $\bullet$ & $\bullet$ & $\bullet$ & $\bullet$ & $\bullet$ & $\bullet$ & $\bullet$ & $\bullet$ & $\bullet$ & $\bullet$ & $\bullet$ & $\bullet$ & $\bullet$ & $\bullet$ & $\bullet$ & $\bullet$ & $\bullet$ & $\bullet$ & $\bullet$ & $\bullet$ & $\bullet$ & $\bullet$ & $\bullet$ & $\bullet$ & $\bullet$ & $\bullet$ & $\bullet$ \\
\hline 04105700 & $\bullet$ & $\bullet$ & $\bullet$ & $\bullet$ & $\bullet$ & $\bullet$ & $\bullet$ & - & $\bullet$ & $\bullet$ & $\bullet$ & $\bullet$ & $\bullet$ & $\bullet$ & $\bullet$ & • & $\bullet$ & $\bullet$ & $\bullet$ & $\bullet$ & $\bullet$ & $\bullet$ & $\bullet$ & $\bullet$ & $\bullet$ & $\bullet$ & $\bullet$ & $\bullet$ & $\bullet$ & $\bullet$ \\
\hline \multicolumn{31}{|l|}{04105800} \\
\hline 04106000 & & & 0 & $\bullet$ & $\bullet$ & $\bullet$ & $\bullet$ & $\bullet$ & $\bullet$ & $\bullet$ & $\bullet$ & $\bullet$ & $\bullet$ & $\bullet$ & $\bullet$ & $\bullet$ & $\bullet$ & $\bullet$ & $\bullet$ & $\bullet$ & $\bullet$ & $\bullet$ & $\bullet$ & $\bullet$ & $\bullet$ & $\bullet$ & $\bullet$ & $\bullet$ & $\bullet$ & $\bullet$ \\
\hline 04106180 & O & 0 & 0 & $\circ$ & 0 & 0 & 0 & O & 0 & $\circ$ & $\circ$ & O & $\circ$ & $\circ$ & 0 & O & $\circ$ & O & $\circ$ & $\circ$ & o & 0 & O & 0 & & & & & & \\
\hline \multicolumn{31}{|l|}{04106190} \\
\hline 04106300 & $\bullet$ & $\bullet$ & $\bullet$ & $\bullet$ & $\bullet$ & $\bullet$ & $\bullet$ & $\bullet$ & $\bullet$ & $\bullet$ & $\bullet$ & $\bullet$ & $\bullet$ & $\bullet$ & $\bullet$ & $\bullet$ & $\bullet$ & $\bullet$ & $\bullet$ & $\bullet$ & $\bullet$ & $\bullet$ & $\bullet$ & $\bullet$ & $\bullet$ & $\bullet$ & & & & \\
\hline 04106320 & 0 & 0 & 0 & 0 & 0 & 0 & 0 & 0 & 0 & 0 & 0 & 0 & 0 & 0 & 0 & 0 & 0 & 0 & 0 & 0 & 0 & 0 & 0 & 0 & 0 & 0 & & & & \\
\hline 04106400 & O & O & 0 & 0 & 0 & 0 & $\circ$ & ० & $\circ$ & $\circ$ & $\circ$ & 0 & $\circ$ & $\circ$ & 0 & o & $\circ$ & 0 & $\circ$ & $\circ$ & o & 0 & 0 & 0 & 0 & $\circ$ & O & 0 & 0 & o \\
\hline 04106500 & 0 & 0 & 0 & 0 & & & & & & & & & & & & & & & & & & & & & & & & & & \\
\hline 04106906 & & & & & & & & & & & & & & & & & & & 0 & $\circ$ & 0 & $\circ$ & 0 & & & & & & & \\
\hline 04107850 & & & & & & & & & & & & & & & & & & & 0 & 0 & 0 & 0 & 0 & & & & & & & \\
\hline 04108500 & $\bullet$ & $\bullet$ & $\bullet$ & $\bullet$ & $\bullet$ & $\bullet$ & $\bullet$ & $\bullet$ & $\bullet$ & $\bullet$ & $\bullet$ & & & & & & & & & & & & & & & & & & & \\
\hline 04108600 & $\bullet$ & $\bullet$ & $\bullet$ & $\bullet$ & $\bullet$ & $\bullet$ & $\bullet$ & $\bullet$ & $\bullet$ & $\bullet$ & $\bullet$ & $\bullet$ & $\bullet$ & $\bullet$ & $\bullet$ & $\bullet$ & $\bullet$ & $\bullet$ & $\bullet$ & $\bullet$ & $\bullet$ & $\bullet$ & $\bullet$ & $\bullet$ & $\bullet$ & $\bullet$ & $\bullet$ & $\bullet$ & $\bullet$ & $\bullet$ \\
\hline 04108670 & & & & & & & & & & & & & 0 & & & & & & & & 0 & 0 & 0 & 0 & 0 & $\circ$ & ○ & 0 & O & ० \\
\hline 04108800 & o & 0 & 0 & 0 & 0 & 0 & 0 & o & 0 & 0 & $\circ$ & 0 & 0 & 0 & 0 & 0 & 0 & 0 & 0 & 0 & 0 & 0 & 0 & 0 & 0 & 0 & 0 & 0 & 0 & 0 \\
\hline 04108801 & o & $\circ$ & 0 & 0 & 0 & 0 & 0 & O & $\circ$ & $\circ$ & 0 & $\circ$ & 0 & 0 & o & o & o & $\circ$ & $\circ$ & 0 & 0 & 0 & 0 & 0 & 0 & & & & & \\
\hline 04109000 & $\bullet$ & $\bullet$ & $\bullet$ & $\bullet$ & $\bullet$ & $\bullet$ & $\bullet$ & $\bullet$ & $\bullet$ & $\bullet$ & $\bullet$ & $\bullet$ & $\bullet$ & $\bullet$ & $\bullet$ & $\bullet$ & $\bullet$ & $\bullet$ & $\bullet$ & $\bullet$ & $\bullet$ & $\bullet$ & $\bullet$ & $\bullet$ & $\bullet$ & $\bullet$ & $\bullet$ & $\bullet$ & $\bullet$ & $\bullet$ \\
\hline \multicolumn{31}{|l|}{04109500} \\
\hline \multicolumn{31}{|l|}{04110000} \\
\hline 04111000 & & & & & & & & & & & & & & $\bullet$ & $\bullet$ & $\bullet$ & $\bullet$ & $\bullet$ & $\bullet$ & $\bullet$ & $\bullet$ & $\bullet$ & $\bullet$ & $\bullet$ & $\bullet$ & $\bullet$ & $\bullet$ & $\bullet$ & $\bullet$ & $\bullet$ \\
\hline 04111379 & $\bullet$ & $\bullet$ & $\bullet$ & $\bullet$ & $\bullet$ & $\bullet$ & $\bullet$ & & & & & & & & & & & & & $\bullet$ & $\bullet$ & $\bullet$ & $\bullet$ & $\bullet$ & $\bullet$ & $\bullet$ & $\bullet$ & $\bullet$ & $\bullet$ & o \\
\hline 04111500 & $\bullet$ & $\bullet$ & $\bullet$ & $\bullet$ & $\bullet$ & $\bullet$ & $\bullet$ & $\bullet$ & $\bullet$ & $\bullet$ & $\bullet$ & $\bullet$ & $\bullet$ & $\bullet$ & $\bullet$ & $\bullet$ & $\bullet$ & $\bullet$ & $\bullet$ & $\bullet$ & $\bullet$ & $\bullet$ & $\bullet$ & $\bullet$ & $\bullet$ & $\bullet$ & $\bullet$ & $\bullet$ & $\bullet$ & \\
\hline 04112000 & o & $\circ$ & 0 & 0 & 0 & 0 & 0 & ० & 0 & 0 & 0 & o & 0 & 0 & o & 0 & 0 & 0 & 0 & 0 & ० & 0 & 0 & 0 & 0 & 0 & o & 0 & 0 & o \\
\hline 04112500 & $\bullet$ & $\bullet$ & $\bullet$ & $\bullet$ & $\bullet$ & $\bullet$ & $\bullet$ & $\bullet$ & $\bullet$ & $\bullet$ & $\bullet$ & $\bullet$ & $\bullet$ & $\bullet$ & $\bullet$ & $\bullet$ & $\bullet$ & $\bullet$ & $\bullet$ & $\bullet$ & $\bullet$ & $\bullet$ & $\bullet$ & $\bullet$ & $\bullet$ & $\bullet$ & $\bullet$ & $\bullet$ & $\bullet$ & $\bullet$ \\
\hline
\end{tabular}


Table 7. Streamgaging stations considered for analysis of study area 3 (hydrologic subregion 0405 and cataloguing unit 04040001 ) in the U.S. Great Lakes Basin, by water year.-Continued

[, station active and included in the analysis; $\bigcirc$, station active but not included in the analysis]

\begin{tabular}{|c|c|c|c|c|c|c|c|c|c|c|c|c|c|c|c|c|c|c|c|c|c|c|c|c|c|c|c|c|c|c|c|c|}
\hline \multirow{2}{*}{$\begin{array}{l}\text { Station } \\
\text { number }\end{array}$} & \multicolumn{32}{|c|}{ Water year } \\
\hline & 동 & ్ㅗㅇ & శ్ర్ల & 志 & 㖞 & ํㅗㅇ & 동 & 㔛 & 옹 & ஜூ & Б요 & ช్ & ஜூ & 志 & 농 & ஜே & क् & ஜீ & ஜீ: & 옹 & Б & $\underset{\sigma}{\mathbb{2}}$ & ๓̊ & 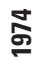 & 모 & $\stackrel{\circ}{\mathscr{\sigma}}$ & ह & $\stackrel{\infty}{\stackrel{\sigma}{\sigma}}$ & న్ & 吕 & চ্口 & ๙ু \\
\hline 04112850 & & & & & & & & & & & & & & & & & & & & & & & & & & $\bullet$ & $\bullet$ & $\bullet$ & $\bullet$ & $\bullet$ & & \\
\hline 04112904 & & & & & & & & & & & & & & & & & & & & & & & & & & - & & & & & & \\
\hline 04113000 & $\bullet$ & $\bullet$ & $\bullet$ & $\bullet$ & $\bullet$ & $\bullet$ & $\bullet$ & $\bullet$ & 0 & $\bullet$ & $\bullet$ & $\bullet$ & $\bullet$ & $\bullet$ & $\bullet$ & $\bullet$ & o & $\bullet$ & $\bullet$ & $\bullet$ & $\bullet$ & $\bullet$ & $\bullet$ & $\bullet$ & $\bullet$ & $\bullet$ & $\bullet$ & 0 & $\bullet$ & $\bullet$ & $\bullet$ & $\bullet$ \\
\hline 04113097 & & & & & & & & & & & & & & & & & & & & & & & & & & $\bullet$ & $\bullet$ & $\bullet$ & $\bullet$ & $\bullet$ & & \\
\hline 04114000 & & & 0 & 0 & $\bullet$ & $\bullet$ & $\bullet$ & $\bullet$ & $\bullet$ & $\bullet$ & $\bullet$ & $\bullet$ & $\bullet$ & $\bullet$ & $\bullet$ & $\bullet$ & $\bullet$ & 0 & $\bullet$ & $\bullet$ & $\bullet$ & $\bullet$ & $\bullet$ & $\bullet$ & $\bullet$ & $\bullet$ & $\bullet$ & $\bullet$ & 0 & $\bullet$ & $\bullet$ & \\
\hline 04114498 & $\bullet$ & $\bullet$ & $\bullet$ & $\bullet$ & $\bullet$ & $\bullet$ & $\bullet$ & $\bullet$ & $\bullet$ & $\bullet$ & $\bullet$ & $\bullet$ & $\bullet$ & $\bullet$ & $\bullet$ & $\bullet$ & $\bullet$ & $\bullet$ & $\bullet$ & $\bullet$ & $\bullet$ & $\bullet$ & $\bullet$ & $\bullet$ & $\bullet$ & $\bullet$ & $\bullet$ & $\bullet$ & $\bullet$ & $\bullet$ & $\bullet$ & $\bullet$ \\
\hline 04114500 & 0 & 0 & 0 & $\circ$ & ० & 0 & 0 & 0 & 0 & o & 0 & 0 & 0 & 0 & ० & 0 & 0 & 0 & 0 & 0 & 0 & 0 & 0 & 0 & 0 & 0 & 0 & 0 & 0 & 0 & 0 & 0 \\
\hline 04115000 & o & ० & o & ○ & $\circ$ & ० & $\circ$ & o & 0 & o & o & ० & o & $\circ$ & $\circ$ & $\circ$ & $\circ$ & ० & O & 0 & 0 & o & o & $\circ$ & $\circ$ & ० & ० & 0 & 0 & o & $\circ$ & O \\
\hline \multicolumn{33}{|l|}{04115265} \\
\hline 04116000 & & $\bullet$ & $\bullet$ & $\bullet$ & $\bullet$ & $\bullet$ & $\bullet$ & $\bullet$ & $\bullet$ & $\bullet$ & $\bullet$ & $\bullet$ & $\bullet$ & $\bullet$ & $\bullet$ & $\bullet$ & $\bullet$ & $\bullet$ & $\bullet$ & $\bullet$ & $\bullet$ & $\bullet$ & $\bullet$ & $\bullet$ & $\bullet$ & $\bullet$ & $\bullet$ & $\bullet$ & $\bullet$ & $\bullet$ & $\bullet$ & $\bullet$ \\
\hline 04116500 & $\bullet$ & $\bullet$ & $\bullet$ & $\bullet$ & $\bullet$ & - & $\bullet$ & $\bullet$ & $\bullet$ & $\bullet$ & $\bullet$ & $\bullet$ & $\bullet$ & $\bullet$ & $\bullet$ & $\bullet$ & $\bullet$ & $\bullet$ & $\bullet$ & $\bullet$ & $\bullet$ & $\bullet$ & $\bullet$ & $\bullet$ & $\bullet$ & $\bullet$ & $\bullet$ & $\bullet$ & $\bullet$ & $\bullet$ & $\bullet$ & $\bullet$ \\
\hline 04117000 & & & & & $\bullet$ & $\bullet$ & $\bullet$ & $\bullet$ & $\bullet$ & $\bullet$ & $\bullet$ & $\bullet$ & $\bullet$ & $\bullet$ & $\bullet$ & $\bullet$ & $\bullet$ & $\bullet$ & $\bullet$ & $\bullet$ & $\bullet$ & $\bullet$ & $\bullet$ & $\bullet$ & $\bullet$ & & & & & & & \\
\hline 04117500 & $\bullet$ & $\bullet$ & $\bullet$ & $\bullet$ & $\bullet$ & $\bullet$ & $\bullet$ & $\bullet$ & $\bullet$ & $\bullet$ & $\bullet$ & $\bullet$ & $\bullet$ & $\bullet$ & $\bullet$ & $\bullet$ & $\bullet$ & $\bullet$ & $\bullet$ & $\bullet$ & $\bullet$ & $\bullet$ & $\bullet$ & $\bullet$ & $\bullet$ & $\bullet$ & $\bullet$ & $\bullet$ & $\bullet$ & $\bullet$ & $\bullet$ & $\bullet$ \\
\hline 04118000 & & $\bullet$ & $\bullet$ & $\bullet$ & $\bullet$ & $\bullet$ & $\bullet$ & $\bullet$ & $\bullet$ & $\bullet$ & $\bullet$ & $\bullet$ & - & $\bullet$ & $\bullet$ & $\bullet$ & $\bullet$ & $\bullet$ & $\bullet$ & $\bullet$ & $\bullet$ & $\bullet$ & $\bullet$ & $\bullet$ & $\bullet$ & $\bullet$ & $\bullet$ & $\bullet$ & $\bullet$ & $\bullet$ & $\bullet$ & \\
\hline 04118500 & & & $\bullet$ & $\bullet$ & $\bullet$ & $\bullet$ & $\bullet$ & $\bullet$ & $\bullet$ & $\bullet$ & $\bullet$ & $\bullet$ & $\bullet$ & $\bullet$ & $\bullet$ & $\bullet$ & $\bullet$ & $\bullet$ & $\bullet$ & $\bullet$ & $\bullet$ & $\bullet$ & $\bullet$ & $\bullet$ & $\bullet$ & $\bullet$ & $\bullet$ & $\bullet$ & $\bullet$ & $\bullet$ & $\bullet$ & $\bullet$ \\
\hline 04119000 & $\bullet$ & $\bullet$ & $\bullet$ & $\bullet$ & $\bullet$ & $\bullet$ & o & $\bullet$ & $\bullet$ & o & $\bullet$ & $\bullet$ & $\bullet$ & $\bullet$ & $\bullet$ & $\bullet$ & ० & o & $\bullet$ & $\bullet$ & o & $\bullet$ & $\bullet$ & $\bullet$ & $\bullet$ & $\bullet$ & $\bullet$ & $\bullet$ & $\bullet$ & $\bullet$ & $\circ$ & $\bullet$ \\
\hline 04119300 & & & & & & & & & & & & & & & & & & & & & & & & & & & $\bullet$ & & & & & \\
\hline
\end{tabular}


Table 7. Streamgaging stations considered for analysis of study area 3 (hydrologic subregion 0405 and cataloguing unit 04040001 ) in the U.S. Great Lakes Basin, by water year.-Continued

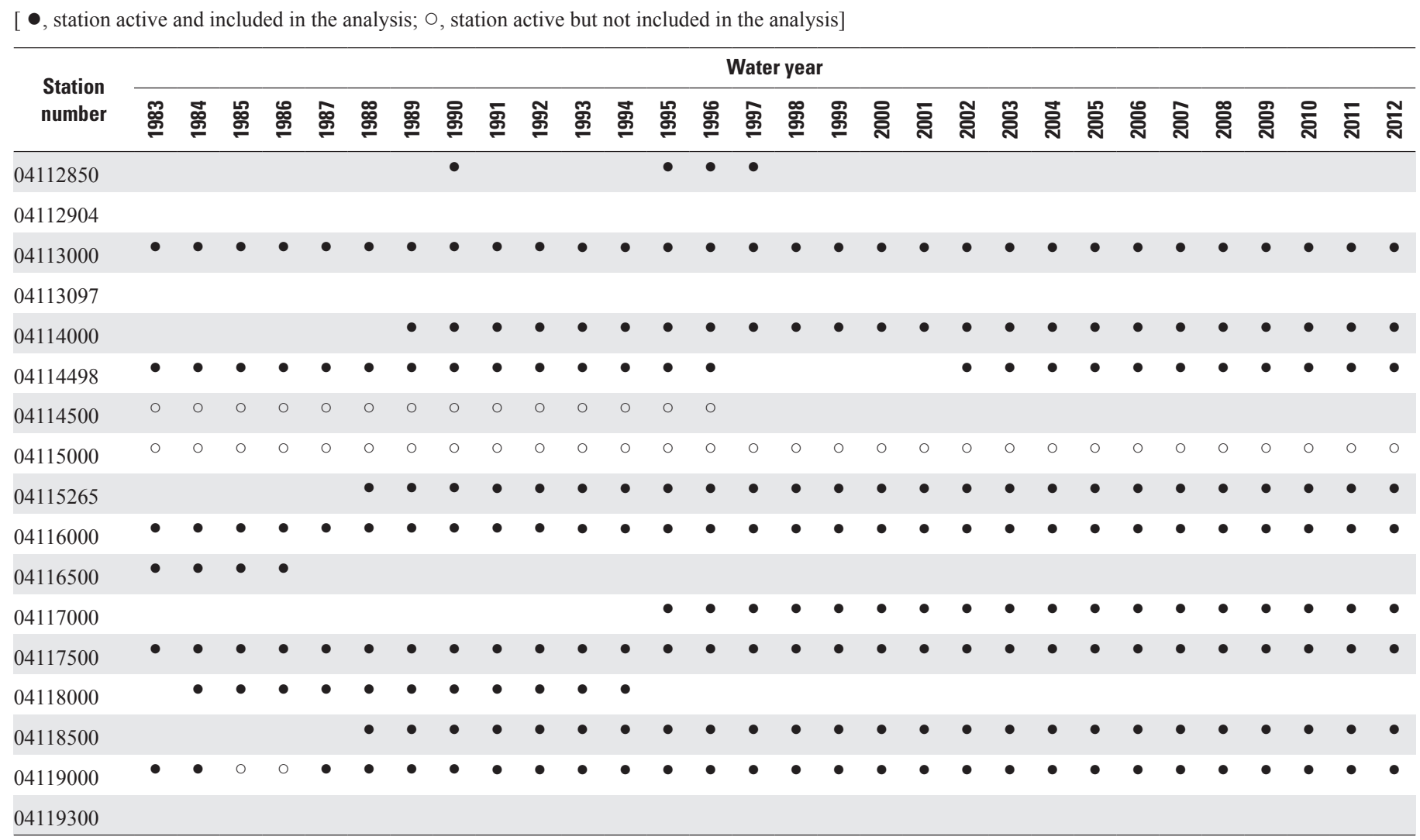


Table 8. Regression statistics and explanatory variables used in the AFINCH (Analysis of Flows In Networks of CHannels) analysis for study area 3 (hydrologic subregion 0405 and cataloguing unit 04040001 ) in the U.S. Great Lakes Basin, by month.

[-, indicates variable not included in monthly regression equation; GDD, growing degree days; RMSE, root mean square error; $\mathrm{R}^{2}$, coefficient of determination. See appendix tables for variable descriptions]

\begin{tabular}{|c|c|c|c|c|c|c|c|c|c|c|c|c|c|c|c|c|c|}
\hline \multirow[b]{2}{*}{ Month } & \multicolumn{14}{|c|}{ Regression coefficients } & \multicolumn{3}{|c|}{ Regression fit statistics } \\
\hline & Intercept & $\begin{array}{l}\text { Current } \\
\text { precipi- } \\
\text { tation }\end{array}$ & $\begin{array}{l}\text { Preceding } \\
\text { precipita- } \\
\text { tion }\end{array}$ & $\begin{array}{c}\text { Current } \\
\text { tempera- } \\
\text { ture }\end{array}$ & $\begin{array}{c}\text { Pasture } \\
\text { hay }\end{array}$ & $\begin{array}{l}\text { Row } \\
\text { crops }\end{array}$ & Forest & $\begin{array}{l}\text { Open } \\
\text { water }\end{array}$ & $\begin{array}{c}\text { Wetland } \\
\text { herbaceous }\end{array}$ & $\begin{array}{l}\text { Stream } \\
\text { order }\end{array}$ & $\begin{array}{c}\text { Mean } \\
\text { elevation }\end{array}$ & $\begin{array}{l}\text { Mean } \\
\text { slope }\end{array}$ & $\begin{array}{l}\text { Coarse } \\
\text { outwash }\end{array}$ & $\begin{array}{c}\text { Average } \\
\text { GDD }\end{array}$ & RMSE & F-statistic & $\mathbf{R}^{2}$ \\
\hline October & 0.529 & 0.091 & 0.055 & - & -0.289 & - & 0.277 & -2.622 & - & $-2.2 \mathrm{E}-05$ & 0.110 & 0.077 & 0.501 & - & 0.181 & 496.8 & 0.641 \\
\hline November & 0.648 & 0.103 & 0.065 & -0.011 & -0.334 & - & 0.490 & -1.802 & -2.646 & $-2.0 \mathrm{E}-05$ & 0.065 & - & 0.359 & $4.8 \mathrm{E}-05$ & 0.181 & 355.4 & 0.609 \\
\hline December & 0.710 & 0.110 & 0.074 & 0.016 & -0.337 & - & 0.545 & -1.131 & -3.075 & $-1.4 \mathrm{E}-05$ & - & - & 0.236 & $4.5 \mathrm{E}-05$ & 0.199 & 296.8 & 0.542 \\
\hline January & 0.805 & 0.120 & 0.104 & 0.032 & -0.286 & - & 0.305 & - & -4.094 & $-1.2 \mathrm{E}-05$ & - & - & 0.183 & 4.9E-05 & 0.223 & 315.5 & 0.531 \\
\hline February & 0.972 & 0.155 & 0.049 & 0.021 & -0.586 & - & - & -1.331 & -3.384 & $-1.3 \mathrm{E}-05$ & 0.042 & - & 0.145 & $5.0 \mathrm{E}-05$ & 0.228 & 202.8 & 0.447 \\
\hline March & 1.161 & 0.118 & 0.094 & - & -0.331 & - & - & -2.131 & - & $-7.0 \mathrm{E}-06$ & - & - & -0.057 & - & 0.235 & 227.4 & 0.352 \\
\hline April & 1.039 & 0.080 & 0.070 & -0.013 & -0.568 & - & - & -1.655 & - & - & - & - & - & - & 0.214 & 209.4 & 0.294 \\
\hline May & 0.818 & 0.094 & 0.074 & -0.015 & -0.439 & - & - & -2.226 & - & $-9.0 \mathrm{E}-06$ & 0.043 & 0.090 & 0.202 & - & 0.184 & 354.7 & 0.560 \\
\hline June & 0.412 & 0.103 & 0.072 & - & -0.593 & - & - & -2.306 & -2.858 & $-1.2 \mathrm{E}-05$ & 0.070 & 0.082 & 0.355 & - & 0.201 & 362.8 & 0.566 \\
\hline July & 0.372 & 0.069 & 0.066 & - & -0.661 & -0.115 & - & -2.336 & -4.678 & $-1.5 \mathrm{E}-05$ & 0.099 & 0.127 & 0.472 & - & 0.17 & 335.5 & 0.572 \\
\hline August & 0.449 & 0.057 & 0.058 & - & -0.595 & -0.187 & - & -2.584 & -4.794 & $-1.9 \mathrm{E}-05$ & 0.120 & 0.139 & 0.509 & - & 0.171 & 305.6 & 0.549 \\
\hline September & 0.711 & 0.076 & 0.047 & -0.016 & -0.571 & -0.172 & - & -2.538 & -5.036 & $-2.2 \mathrm{E}-05$ & 0.114 & 0.129 & 0.523 & $2.9 \mathrm{E}-05$ & 0.171 & 355.1 & 0.630 \\
\hline
\end{tabular}

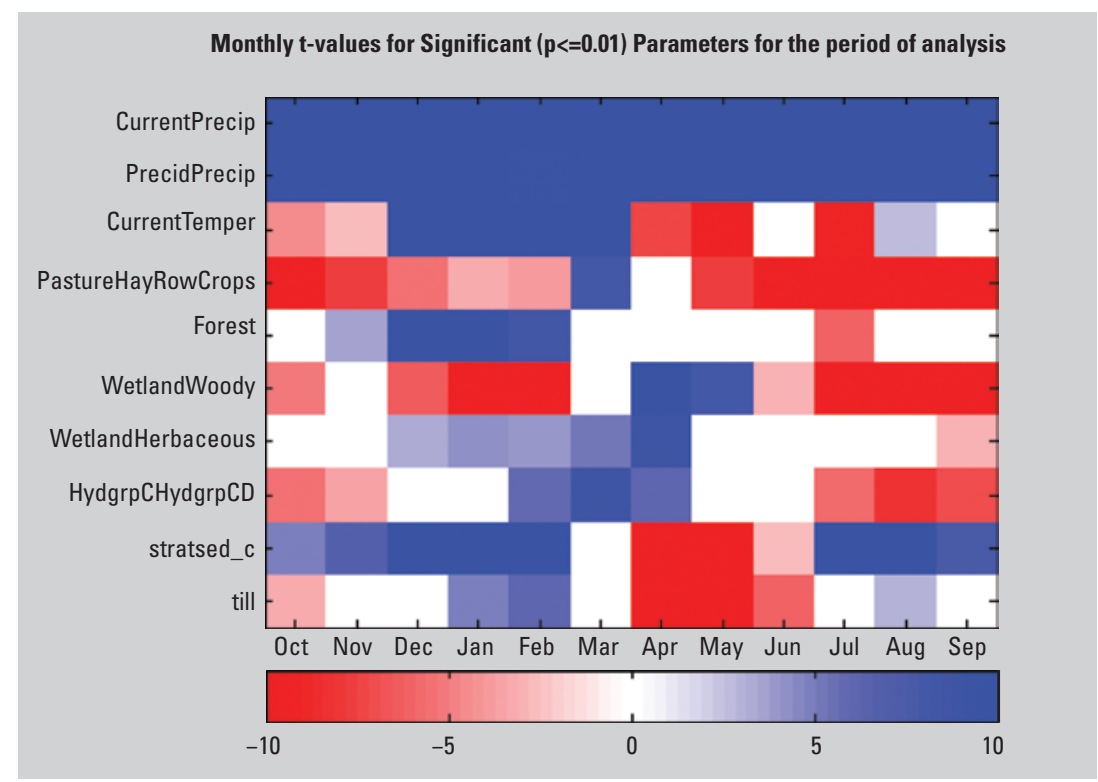

Figure 9. Image showing the t-value-indexed colors corresponding to each explanatory variable in the monthly regression equation for estimating water yield for study area 3 (hydrologic subregion 0405 and cataloguing unit 04040001) in the U.S. Great Lakes Basin. 


\section{Study Area 4}

Study area 4 is formed by cataloguing units 04060101 , 04060102, 04060103, 04060104, 04060105, 04070003, 04070004, 04070005, 04070006, and 04070007 near the central part of the U.S. Great Lakes Basin in the northern part of the Lower Peninsula of Michigan (fig. 10). The number of stations included in the analysis ranged from 15 (1994) to
30 (1970) (table 9). Seven explanatory variables were selected during the regression analysis with the number of variables specified for each monthly equation ranging from 5 (May) to 7 (December, January, February, and July) (table 3). Variables and regression statistics based on the stepwise regression for each month are indicated in table 10 and figure 11.

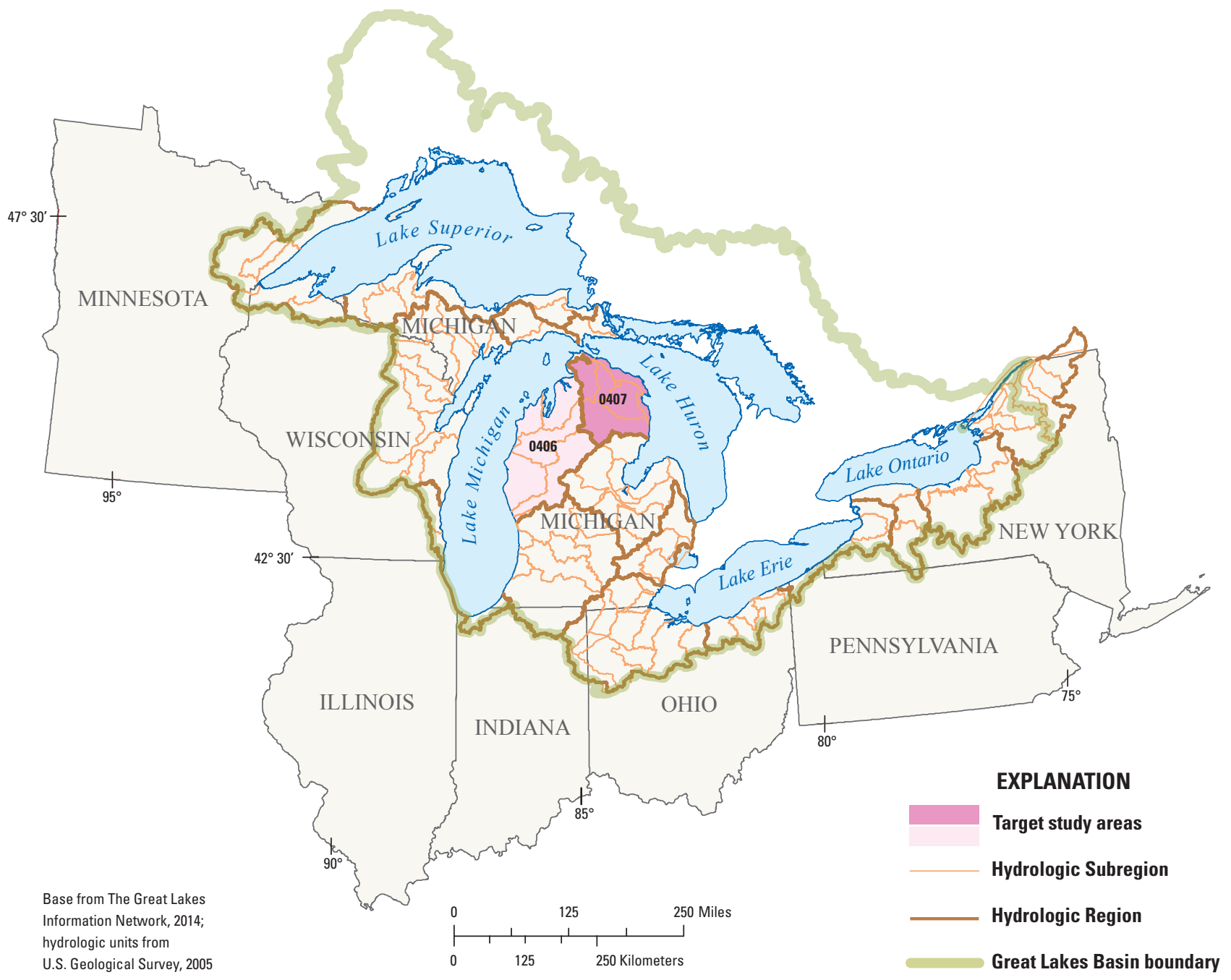

Figure 10. Location of study area 4 (cataloguing units $04060101,04060102,04060103,04060104,04060105,04070003,04070004$, 04070005, 04070006, and 04070007) in the U.S. Great Lakes Basin. 
Table 9. Streamgaging stations considered for analysis of study area 4 (cataloguing units $04060101,04060102,04060103,04060104,04060105$, 04070003, 04070004, 04070005, 04070006, and 04070007) in the U.S. Great Lakes Basin, by water year.-Continued

[ $\bullet$, station active and included in the analysis; $\circ$, station active but not included in the analysis]

\begin{tabular}{|c|c|c|c|c|c|c|c|c|c|c|c|c|c|c|c|c|c|c|c|c|c|c|c|c|c|c|c|c|c|c|c|c|}
\hline \multirow{2}{*}{$\begin{array}{l}\text { Station } \\
\text { number }\end{array}$} & \multicolumn{32}{|c|}{ Water year } \\
\hline & ్ㅗㅇ & ్ํㅀ & ஜึ్ & 냉 & 농 & 号 & 옹 & 骂 & 骂 & ६్ & $\bar{\varnothing}$ & ชั & ஜூ & छ & 용 & ६ & 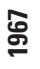 & 吅 & 。্̊ & 욤 & $\frac{5}{2}$ & $\underset{\frac{N}{\sigma}}{\frac{N}{\sigma}}$ & ๓̊ & $\frac{\pi}{2}$ & 络 & $\stackrel{\circ}{\circ}$ & Б & $\stackrel{\infty}{\sigma}$ & $\frac{\sigma}{\sigma}$ & 吕 & ฐ্口 & ঞ \\
\hline 04120293 & & & & & & & & & & & & & & & & & & & & & & & & & $\bullet$ & & & & & & & \\
\hline 04121000 & $\bullet$ & $\bullet$ & $\bullet$ & $\bullet$ & $\bullet$ & $\bullet$ & $\bullet$ & $\bullet$ & $\bullet$ & $\bullet$ & $\bullet$ & $\bullet$ & $\bullet$ & $\bullet$ & $\bullet$ & $\bullet$ & $\bullet$ & $\bullet$ & $\bullet$ & $\bullet$ & $\bullet$ & $\bullet$ & $\bullet$ & & & & & & & & & \\
\hline 04121300 & & & & & & & & & & & & & & & & & $\bullet$ & $\bullet$ & $\bullet$ & $\bullet$ & $\bullet$ & $\bullet$ & $\bullet$ & $\bullet$ & $\bullet$ & $\bullet$ & $\bullet$ & $\bullet$ & $\bullet$ & $\bullet$ & $\bullet$ & $\bullet$ \\
\hline 04121500 & $\bullet$ & $\bullet$ & $\bullet$ & $\bullet$ & $\bullet$ & $\bullet$ & $\bullet$ & $\bullet$ & $\bullet$ & $\bullet$ & $\bullet$ & $\bullet$ & $\bullet$ & $\bullet$ & $\bullet$ & $\bullet$ & $\bullet$ & $\bullet$ & $\bullet$ & $\bullet$ & $\bullet$ & $\bullet$ & $\bullet$ & $\bullet$ & $\bullet$ & $\bullet$ & $\bullet$ & $\bullet$ & $\bullet$ & $\bullet$ & $\bullet$ & $\bullet$ \\
\hline 04121650 & & & & & & & & & & & & & & & & & & & & & & & & & & & & & & & & \\
\hline 04121900 & & & & & & & & & & & & & & & & & $\bullet$ & $\bullet$ & $\bullet$ & $\bullet$ & $\bullet$ & $\bullet$ & $\bullet$ & $\bullet$ & $\bullet$ & $\bullet$ & $\bullet$ & $\bullet$ & $\bullet$ & $\bullet$ & $\bullet$ & $\bullet$ \\
\hline 04121944 & & & & & & & & & & & & & & & & & & & & & & & & & & & & & & & & \\
\hline 04121970 & & & & & & & & & & & & & & & & & & & & & & & & & & & & & & & & \\
\hline 04122000 & $\bullet$ & $\bullet$ & $\bullet$ & $\bullet$ & $\bullet$ & $\bullet$ & $\bullet$ & $\bullet$ & $\bullet$ & $\bullet$ & $\bullet$ & $\bullet$ & $\bullet$ & $\bullet$ & $\bullet$ & $\bullet$ & $\bullet$ & $\bullet$ & $\bullet$ & $\bullet$ & $\bullet$ & $\bullet$ & $\bullet$ & $\bullet$ & $\bullet$ & $\bullet$ & $\bullet$ & $\bullet$ & $\bullet$ & $\bullet$ & $\bullet$ & $\bullet$ \\
\hline 04122100 & & & & & & & & & & & & & & & & $\bullet$ & $\bullet$ & $\bullet$ & $\bullet$ & $\bullet$ & $\bullet$ & $\bullet$ & $\bullet$ & $\bullet$ & $\bullet$ & $\bullet$ & $\bullet$ & $\bullet$ & $\bullet$ & $\bullet$ & $\bullet$ & $\bullet$ \\
\hline 04122150 & & & & & & & & & & & & & & & & & & & & & & & & & & & & & & & & \\
\hline 04122200 & & & & & & & & $\bullet$ & $\bullet$ & $\bullet$ & $\bullet$ & $\bullet$ & $\bullet$ & $\bullet$ & $\bullet$ & $\bullet$ & $\bullet$ & $\bullet$ & $\bullet$ & $\bullet$ & $\bullet$ & $\bullet$ & $\bullet$ & $\bullet$ & $\bullet$ & $\bullet$ & $\bullet$ & $\bullet$ & $\bullet$ & $\bullet$ & $\bullet$ & $\bullet$ \\
\hline 04122500 & $\bullet$ & $\bullet$ & $\bullet$ & $\bullet$ & $\bullet$ & $\bullet$ & $\bullet$ & $\bullet$ & $\bullet$ & $\bullet$ & $\bullet$ & $\bullet$ & $\bullet$ & $\bullet$ & $\bullet$ & $\bullet$ & $\bullet$ & $\bullet$ & $\bullet$ & $\bullet$ & $\bullet$ & $\bullet$ & $\bullet$ & $\bullet$ & $\bullet$ & $\bullet$ & $\bullet$ & $\bullet$ & $\bullet$ & $\bullet$ & $\bullet$ & $\bullet$ \\
\hline 04123000 & $\bullet$ & $\bullet$ & $\bullet$ & $\bullet$ & $\bullet$ & $\bullet$ & $\bullet$ & $\bullet$ & $\bullet$ & $\bullet$ & $\bullet$ & $\bullet$ & $\bullet$ & $\bullet$ & $\bullet$ & $\bullet$ & $\bullet$ & $\bullet$ & $\bullet$ & $\bullet$ & $\bullet$ & $\bullet$ & $\bullet$ & & & & & & & & & \\
\hline 04123500 & $\bullet$ & $\bullet$ & $\bullet$ & $\bullet$ & $\bullet$ & $\bullet$ & $\bullet$ & $\bullet$ & $\bullet$ & $\bullet$ & $\bullet$ & $\bullet$ & $\bullet$ & $\bullet$ & $\bullet$ & $\bullet$ & $\bullet$ & $\bullet$ & $\bullet$ & $\bullet$ & $\bullet$ & $\bullet$ & $\bullet$ & & & & & & & & & \\
\hline 04124000 & $\bullet$ & $\bullet$ & $\bullet$ & $\bullet$ & $\bullet$ & $\bullet$ & $\bullet$ & $\bullet$ & $\bullet$ & $\bullet$ & $\bullet$ & $\bullet$ & $\bullet$ & $\bullet$ & $\bullet$ & $\bullet$ & $\bullet$ & $\bullet$ & $\bullet$ & $\bullet$ & $\bullet$ & $\bullet$ & $\bullet$ & $\bullet$ & $\bullet$ & $\bullet$ & $\bullet$ & $\bullet$ & $\bullet$ & $\bullet$ & $\bullet$ & $\bullet$ \\
\hline 04124200 & & & & & & & & & & & & & & & & & & & & & & & & & & & & & & & & \\
\hline 04124500 & & & $\bullet$ & $\bullet$ & $\bullet$ & $\bullet$ & $\bullet$ & $\bullet$ & $\bullet$ & $\bullet$ & $\bullet$ & $\bullet$ & $\bullet$ & & & & & & & & & & & & & & & & & & & \\
\hline 04125000 & & & $\bullet$ & $\bullet$ & $\bullet$ & $\bullet$ & $\bullet$ & $\bullet$ & $\bullet$ & $\bullet$ & $\bullet$ & $\bullet$ & $\bullet$ & & & & & & & & & & & & & & & & & & & \\
\hline 04125200 & & & & & & & & & & & & & & & & & & $\bullet$ & $\bullet$ & $\bullet$ & & & & & & & & & & & & \\
\hline 04125210 & & & & & & & & & & & & & & & & & & & & $\bullet$ & & & & & & & & & & & & \\
\hline 04125350 & & & & & & & & & & & & & & & & & & & & $\circ$ & & & & & & & & & & & & \\
\hline 04125450 & & & & & & & & & & & & & & & & & & & $\bullet$ & $\bullet$ & & & & & & & & & & & & \\
\hline 04125460 & & & O & 0 & 0 & $\circ$ & $\circ$ & 0 & $\circ$ & $\circ$ & $\circ$ & $\circ$ & 0 & 0 & 0 & $\circ$ & $\circ$ & $\circ$ & $\circ$ & $\circ$ & $\circ$ & 0 & $\circ$ & $\circ$ & 0 & $\circ$ & $\circ$ & 0 & 0 & $\circ$ & 0 & 0 \\
\hline 04125500 & & & $\bullet$ & $\bullet$ & $\bullet$ & $\bullet$ & $\bullet$ & $\bullet$ & $\bullet$ & $\bullet$ & $\bullet$ & $\bullet$ & $\bullet$ & $\bullet$ & $\bullet$ & $\bullet$ & $\bullet$ & $\bullet$ & $\bullet$ & $\bullet$ & $\bullet$ & $\bullet$ & $\bullet$ & $\bullet$ & $\bullet$ & $\bullet$ & $\bullet$ & $\bullet$ & $\bullet$ & $\bullet$ & $\bullet$ & $\bullet$ \\
\hline 04125510 & & & & & & & & & & & & & & & & & & $\circ$ & $\circ$ & $\circ$ & & & & & & & & & & & & \\
\hline 04125550 & & & & & & & & & & & & & & & & & & & & & & & & & & & & & & & & \\
\hline 04126000 & & $\bullet$ & $\bullet$ & $\bullet$ & $\bullet$ & $\bullet$ & $\bullet$ & $\bullet$ & $\bullet$ & $\bullet$ & $\bullet$ & $\bullet$ & $\bullet$ & $\bullet$ & $\bullet$ & $\bullet$ & $\bullet$ & $\bullet$ & $\bullet$ & $\bullet$ & $\bullet$ & $\bullet$ & $\bullet$ & $\bullet$ & $\bullet$ & $\bullet$ & $\bullet$ & $\bullet$ & $\bullet$ & $\bullet$ & $\bullet$ & $\bullet$ \\
\hline 04126200 & & & & & & & $\bullet$ & $\bullet$ & $\bullet$ & $\bullet$ & $\bullet$ & $\bullet$ & $\bullet$ & $\bullet$ & $\bullet$ & $\bullet$ & $\bullet$ & $\bullet$ & $\bullet$ & $\bullet$ & $\bullet$ & $\bullet$ & $\bullet$ & $\bullet$ & $\bullet$ & & & & & & & \\
\hline 04126740 & & & & & & & & & & & & & & & & & & & & & & & & & & & & & & & & \\
\hline 04126802 & & & & & & & & & & & & & & & & & & & & & & & & & & & & & & & & \\
\hline 04126970 & & & & & & & & & & & & & & & & & & & & & & & & & & & & & & & & \\
\hline 04127000 & & & $\bullet$ & $\bullet$ & $\bullet$ & $\bullet$ & $\bullet$ & $\bullet$ & $\bullet$ & $\bullet$ & $\bullet$ & $\bullet$ & $\bullet$ & $\bullet$ & $\bullet$ & $\bullet$ & $\bullet$ & $\bullet$ & $\bullet$ & $\bullet$ & $\bullet$ & $\bullet$ & $\bullet$ & $\bullet$ & $\bullet$ & $\bullet$ & $\bullet$ & $\bullet$ & $\bullet$ & $\bullet$ & $\bullet$ & $\bullet$ \\
\hline 04127565 & & & & & & & & & & & & & & & & & & & & & & & & & & & & & & & & \\
\hline 04127800 & & & & & & & & & & & & & & & & & O & ○ & 0 & 0 & $\circ$ & 0 & $\circ$ & $\circ$ & $\circ$ & $\circ$ & $\circ$ & ○ & ० & 0 & $\circ$ & $\circ$ \\
\hline 04127997 & $\circ$ & 0 & 0 & 0 & $\circ$ & 0 & $\circ$ & $\circ$ & $\circ$ & $\circ$ & $\circ$ & $\circ$ & 0 & 0 & 0 & 0 & 0 & $\circ$ & $\circ$ & $\circ$ & 0 & 0 & $\circ$ & 0 & 0 & 0 & 0 & 0 & 0 & 0 & $\circ$ & 0 \\
\hline 04128000 & $\bullet$ & $\bullet$ & $\bullet$ & $\bullet$ & $\bullet$ & $\bullet$ & $\bullet$ & $\bullet$ & $\bullet$ & $\bullet$ & $\bullet$ & $\bullet$ & $\bullet$ & $\bullet$ & $\bullet$ & $\bullet$ & $\bullet$ & $\bullet$ & $\bullet$ & $\bullet$ & $\bullet$ & $\bullet$ & $\bullet$ & $\bullet$ & $\bullet$ & $\bullet$ & $\bullet$ & $\bullet$ & $\bullet$ & $\bullet$ & $\bullet$ & $\bullet$ \\
\hline 04128500 & $\bullet$ & $\bullet$ & $\bullet$ & $\bullet$ & $\bullet$ & $\bullet$ & $\bullet$ & $\bullet$ & $\bullet$ & $\bullet$ & $\bullet$ & $\bullet$ & $\bullet$ & $\bullet$ & $\bullet$ & $\bullet$ & $\bullet$ & $\bullet$ & $\bullet$ & $\bullet$ & $\bullet$ & $\bullet$ & $\bullet$ & $\bullet$ & $\bullet$ & $\bullet$ & $\bullet$ & $\bullet$ & $\bullet$ & $\bullet$ & $\bullet$ & $\bullet$ \\
\hline 04128990 & $\bullet$ & $\bullet$ & $\bullet$ & $\bullet$ & $\bullet$ & $\bullet$ & $\bullet$ & $\bullet$ & $\bullet$ & $\bullet$ & $\bullet$ & $\bullet$ & $\bullet$ & $\bullet$ & $\bullet$ & $\bullet$ & $\bullet$ & 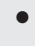 & $\bullet$ & $\bullet$ & $\bullet$ & $\bullet$ & $\bullet$ & $\bullet$ & $\bullet$ & $\bullet$ & $\bullet$ & 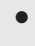 & 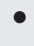 & $\bullet$ & $\bullet$ & $\bullet$ \\
\hline
\end{tabular}


Table 9. Streamgaging stations considered for analysis of study area 4 (cataloguing units 04060101, 04060102, 04060103, 04060104, 04060105, 04070003, 04070004, 04070005, 04070006, and 04070007) in the U.S. Great Lakes Basin, by water year.-Continued

$[\bullet$, station active and included in the analysis; $\circ$, station active but not included in the analysis]

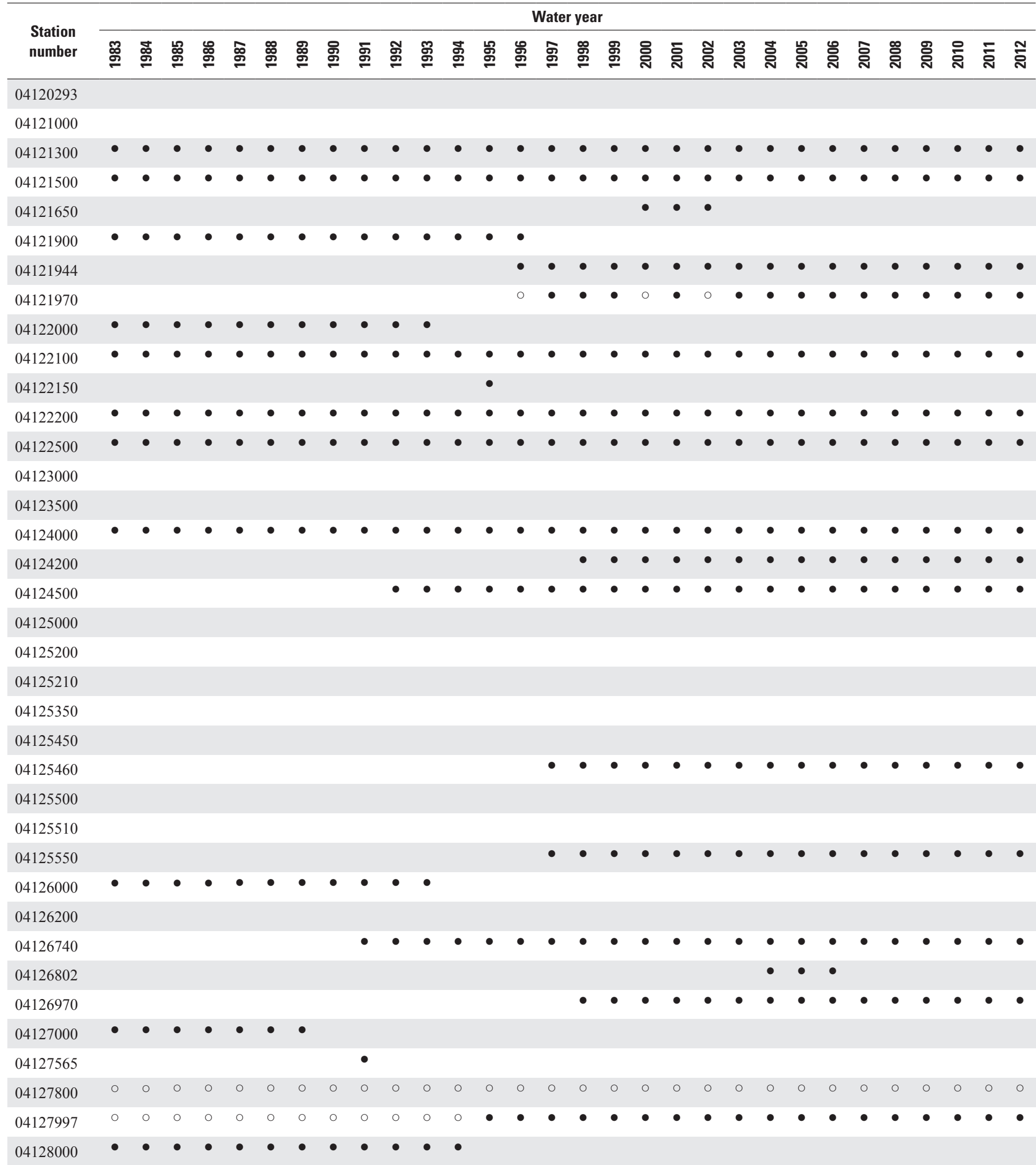


Table 9. Streamgaging stations considered for analysis of study area 4 (cataloguing units 04060101, 04060102, 04060103, 04060104, 04060105, 04070003, 04070004, 04070005, 04070006, and 04070007) in the U.S. Great Lakes Basin, by water year.-Continued

[ $\bullet$, station active and included in the analysis; ○, station active but not included in the analysis]

\begin{tabular}{|c|c|c|c|c|c|c|c|c|c|c|c|c|c|c|c|c|c|c|c|c|c|c|c|c|c|c|c|c|c|c|c|c|}
\hline \multirow{2}{*}{$\begin{array}{l}\text { Station } \\
\text { number }\end{array}$} & \multicolumn{32}{|c|}{ Water year } \\
\hline & ్ㅗㅇ & స్్ㅁ & ஜ్ & 壱 & 败 & 兽 & ફ્ & 吕 & 윰 & ळ్ & $\Phi$ & ๘ & $\dddot{ஜ}$ & ‡్ & 岕 & ஜ્ & ळ్ & ஜ̊ & 。ㅇ & 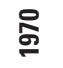 & $\underset{\sigma}{\sigma}$ & $\underset{\mathrm{N}}{\mathrm{N}}$ & $\stackrel{\Re}{\mathscr{\sigma}}$ & $\frac{\pi}{2}$ & $\stackrel{\text { L }}{\frac{2}{\sigma}}$ & $\stackrel{\varrho}{\mathscr{\sigma}}$ & હ & $\stackrel{\infty}{\sigma}$ & 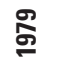 & 吕 & ळ & ळ \\
\hline 04129000 & $\circ$ & o & o & $\circ$ & $\circ$ & ० & o & o & $\circ$ & $\circ$ & $\circ$ & ० & o & $\circ$ & 0 & $\circ$ & $\circ$ & 0 & 0 & $\circ$ & o & 0 & $\circ$ & ० & ० & ० & $\circ$ & $\circ$ & ० & $\circ$ & $\circ$ & o \\
\hline 04129500 & $\bullet$ & $\bullet$ & $\bullet$ & $\bullet$ & - & $\bullet$ & - & - & $\bullet$ & $\bullet$ & - & $\bullet$ & $\bullet$ & $\bullet$ & $\bullet$ & $\bullet$ & - & $\bullet$ & $\bullet$ & $\bullet$ & - & - & - & $\bullet$ & $\bullet$ & $\bullet$ & $\bullet$ & $\bullet$ & - & - & $\bullet$ & \\
\hline 04130000 & o & O & O & $\circ$ & O & O & O & o & 0 & $\circ$ & O & O & ○ & ○ & O & O & o & O & o & O & O & O & 0 & O & O & O & $\circ$ & $\circ$ & O & O & $\circ$ & o \\
\hline 04130500 & $\bullet$ & $\bullet$ & $\bullet$ & $\bullet$ & $\bullet$ & $\bullet$ & - & - & $\bullet$ & - & - & $\bullet$ & $\bullet$ & $\bullet$ & $\bullet$ & - & - & $\bullet$ & $\bullet$ & $\bullet$ & - & $\bullet$ & $\bullet$ & $\bullet$ & $\bullet$ & • & $\bullet$ & $\bullet$ & - & - & $\bullet$ & $\bullet$ \\
\hline 04131000 & $\bullet$ & $\bullet$ & & & & & & & & & & & & & & & & & & & & & & & & & & & & & & \\
\hline 04131500 & & & $\bullet$ & $\bullet$ & $\bullet$ & $\bullet$ & $\bullet$ & $\bullet$ & $\bullet$ & $\bullet$ & $\bullet$ & $\bullet$ & $\bullet$ & $\bullet$ & $\bullet$ & $\bullet$ & $\bullet$ & $\bullet$ & $\bullet$ & $\bullet$ & $\bullet$ & $\bullet$ & $\bullet$ & $\bullet$ & $\bullet$ & $\bullet$ & $\bullet$ & $\bullet$ & $\bullet$ & & & \\
\hline 04132000 & O & O & O & $\circ$ & O & O & O & O & 0 & $\circ$ & O & O & ○ & O & O & O & o & O & O & O & O & O & O & O & & & & & & & & \\
\hline 04132500 & $\bullet$ & $\bullet$ & $\bullet$ & $\bullet$ & - & $\bullet$ & - & - & $\bullet$ & - & - & $\bullet$ & $\bullet$ & $\bullet$ & $\bullet$ & • & - & $\bullet$ & $\bullet$ & - & $\bullet$ & $\bullet$ & & & & & & & & & & \\
\hline 04133000 & $\bullet$ & $\bullet$ & $\bullet$ & & & & & & & & & & & & & & & & & & & & & & & & & & & & & \\
\hline 04133500 & o & 0 & O & $\circ$ & 0 & 0 & 0 & 0 & o & $\circ$ & 0 & 0 & 0 & o & 0 & o & o & O & O & 0 & O & ○ & 0 & 0 & o & O & $\circ$ & $\circ$ & 0 & ○ & & \\
\hline 04133501 & $\bullet$ & $\bullet$ & $\bullet$ & $\bullet$ & $\bullet$ & $\bullet$ & $\bullet$ & $\bullet$ & $\bullet$ & $\bullet$ & $\bullet$ & $\bullet$ & $\bullet$ & $\bullet$ & $\bullet$ & $\bullet$ & $\bullet$ & $\bullet$ & $\bullet$ & $\bullet$ & $\bullet$ & $\bullet$ & $\bullet$ & $\bullet$ & $\bullet$ & $\bullet$ & $\bullet$ & $\bullet$ & $\bullet$ & $\bullet$ & & \\
\hline 04134000 & ० & 0 & 0 & ० & 0 & 0 & ० & 0 & 0 & $\circ$ & 0 & 0 & ○ & O & 0 & O & 0 & 0 & ○ & 0 & 0 & 0 & 0 & 0 & 0 & 0 & 0 & $\circ$ & 0 & 0 & & \\
\hline 04134500 & $\bullet$ & $\bullet$ & $\bullet$ & & & & & & & & & & & & & & & & & & & & & & & & & & & & & \\
\hline 04135000 & & & & & & & & & & & & & & & & & & & & & & & & & & & & & & $\bullet$ & $\bullet$ & $\bullet$ \\
\hline 04135500 & $\bullet$ & $\bullet$ & $\bullet$ & $\bullet$ & $\bullet$ & $\bullet$ & $\bullet$ & $\bullet$ & $\bullet$ & $\bullet$ & $\bullet$ & $\bullet$ & $\bullet$ & $\bullet$ & $\bullet$ & $\bullet$ & $\bullet$ & $\bullet$ & $\bullet$ & $\bullet$ & $\bullet$ & $\bullet$ & $\bullet$ & $\bullet$ & $\bullet$ & $\bullet$ & $\bullet$ & $\bullet$ & $\bullet$ & $\bullet$ & $\bullet$ & $\bullet$ \\
\hline 04135600 & & & & & & & & & $\bullet$ & $\bullet$ & $\bullet$ & $\bullet$ & $\bullet$ & $\bullet$ & $\bullet$ & $\bullet$ & $\bullet$ & $\bullet$ & $\bullet$ & $\bullet$ & $\bullet$ & $\bullet$ & $\bullet$ & $\bullet$ & $\bullet$ & $\bullet$ & $\bullet$ & $\bullet$ & $\bullet$ & $\bullet$ & $\bullet$ & $\bullet$ \\
\hline 04135700 & & & & & & & & & & & & & & & & & $\bullet$ & $\bullet$ & $\bullet$ & $\bullet$ & $\bullet$ & $\bullet$ & $\bullet$ & $\bullet$ & $\bullet$ & $\bullet$ & $\bullet$ & $\bullet$ & $\bullet$ & $\bullet$ & $\bullet$ & $\bullet$ \\
\hline \multicolumn{33}{|l|}{04136000} \\
\hline 04136500 & & & $\bullet$ & $\bullet$ & $\bullet$ & $\bullet$ & $\bullet$ & $\bullet$ & $\bullet$ & $\bullet$ & $\bullet$ & $\bullet$ & $\bullet$ & $\bullet$ & $\bullet$ & $\bullet$ & $\bullet$ & $\bullet$ & $\bullet$ & $\bullet$ & $\bullet$ & $\bullet$ & $\bullet$ & $\bullet$ & $\bullet$ & $\bullet$ & $\bullet$ & $\bullet$ & $\bullet$ & $\bullet$ & $\bullet$ & $\bullet$ \\
\hline \multicolumn{33}{|l|}{04136900} \\
\hline \multicolumn{33}{|l|}{04137005} \\
\hline 04137500 & & & & & & & & & & & & & & & & & & & & & & & & & & & & & & & & \\
\hline
\end{tabular}


Table 9. Streamgaging stations considered for analysis of study area 4 (cataloguing units 04060101, 04060102, 04060103, 04060104, 04060105, 04070003, 04070004, 04070005, 04070006, and 04070007) in the U.S. Great Lakes Basin, by water year.-Continued

[ $\bullet$, station active and included in the analysis; $\circ$, station active but not included in the analysis]

\begin{tabular}{|c|c|c|c|c|c|c|c|c|c|c|c|c|c|c|c|c|c|c|c|c|c|c|c|c|c|c|c|c|c|c|}
\hline \multirow{2}{*}{$\begin{array}{l}\text { Station } \\
\text { number }\end{array}$} & \multicolumn{30}{|c|}{ Water year } \\
\hline & ஜ & 范 & 농 & ஜ̊ & ळ్ & $\begin{array}{l}\stackrel{\infty}{0} \\
\text { \% }\end{array}$ & 용 & 요 & Б্ & శ్ & ஜூ & 莺 & 둉 & ஜே & જூ & 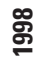 & 욤 & ్్ㅇ & ్ㅠ & ণ్రి & ํํํ & 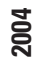 & 농 & 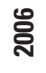 & స్్ & 号 & 윰 & 음 & స్ & $\frac{N}{\delta}$ \\
\hline 04129000 & 0 & O & O & 0 & 0 & $\circ$ & $\circ$ & 0 & & & & & & & & & & & & & & & & & & & & & & \\
\hline \multicolumn{31}{|l|}{04129500} \\
\hline \multicolumn{31}{|l|}{04130000} \\
\hline 04130500 & - & $\bullet$ & $\bullet$ & $\bullet$ & - & - & - & $\bullet$ & $\bullet$ & - & $\bullet$ & $\bullet$ & - & - & - & - & - & $\bullet$ & & & & & & & & & & & & \\
\hline \multicolumn{31}{|l|}{04131000} \\
\hline \multicolumn{31}{|l|}{04131500} \\
\hline \multicolumn{31}{|l|}{04132000} \\
\hline \multicolumn{31}{|l|}{04132500} \\
\hline \multicolumn{31}{|l|}{04133000} \\
\hline \multicolumn{31}{|l|}{04133500} \\
\hline 04133501 & & & & & & & & & & & & & & & & & & & & & - & $\bullet$ & - & - & - & - & - & $\bullet$ & - & - \\
\hline \multicolumn{31}{|l|}{04134000} \\
\hline \multicolumn{31}{|l|}{04134500} \\
\hline 04135000 & - & - & - & - & - & - & - & - & $\bullet$ & • & - & & & & & & & & & & & & & & & & & & & \\
\hline 04135500 & $\bullet$ & - & $\bullet$ & $\bullet$ & $\bullet$ & - & $\bullet$ & $\bullet$ & $\bullet$ & $\bullet$ & - & & & & & & & & & & & & & & & & & & & \\
\hline 04135600 & • & $\bullet$ & & & & & & & & & & & & & & & & & & & & & & & & & & & & \\
\hline 04135700 & $\bullet$ & - & - & - & $\bullet$ & - & - & & $\bullet$ & • & - & - & $\bullet$ & $\bullet$ & - & - & $\bullet$ & $\bullet$ & $\bullet$ & $\bullet$ & - & $\bullet$ & - & - & - & - & • & $\bullet$ & $\bullet$ & - \\
\hline 04136000 & & & & & & & & & & & & & & - & - & - & • & $\bullet$ & - & • & - & - & - & - & - & - & - & • & - & - \\
\hline 04136500 & - & $\bullet$ & - & $\bullet$ & $\bullet$ & - & - & • & $\bullet$ & • & • & • & - & $\bullet$ & - & - & - & $\bullet$ & $\bullet$ & • & - & $\bullet$ & • & - & - & - & • & • & $\bullet$ & • \\
\hline 04136900 & & & & & & & & & & & & & & & - & - & - & $\bullet$ & - & - & - & - & - & - & - & - & - & • & - & - \\
\hline 04137005 & & & & & & & & & & & & & & & 0 & 0 & 0 & 0 & 0 & O & 0 & 0 & O & 0 & O & 0 & 0 & O & O & 0 \\
\hline 04137500 & & & & & & - & - & - & - & - & - & - & - & $\bullet$ & - & - & - & $\bullet$ & - & - & - & - & - & - & - & - & - & $\bullet$ & $\bullet$ & - \\
\hline
\end{tabular}


Table 10. Regression statistics and explanatory variables used in the AFINCH (Analysis of Flows In Networks of CHannels) analysis for study area 4 (cataloguing units 04060101, 04060102, 04060103, 04060104, 04060105, 04070003, 04070004, 04070005, 04070006, and 04070007) in the U.S. Great Lakes Basin, by month.

[-, indicates variable not included in monthly regression equation; RMSE, root mean square error; $\mathrm{R}^{2}$, coefficient of determination. See appendix tables for variable descriptions]

\begin{tabular}{|c|c|c|c|c|c|c|c|c|c|c|c|}
\hline \multirow[b]{2}{*}{ Month } & \multicolumn{8}{|c|}{ Regression coefficients } & \multicolumn{3}{|c|}{ Regression fit statistics } \\
\hline & Intercept & $\begin{array}{l}\text { Current } \\
\text { precipita- } \\
\text { tion }\end{array}$ & $\begin{array}{l}\text { Preceding } \\
\text { precipita- } \\
\text { tion }\end{array}$ & $\begin{array}{c}\text { Current } \\
\text { tempera- } \\
\text { ture }\end{array}$ & $\begin{array}{l}\text { Deciduous } \\
\text { and evergreen } \\
\text { forest }\end{array}$ & $\begin{array}{l}\text { Row } \\
\text { crops }\end{array}$ & $\begin{array}{l}\text { Hydric } \\
\text { group A } \\
\text { and AD }\end{array}$ & $\begin{array}{c}\text { Stream } \\
\text { order }\end{array}$ & RMSE & F-statistic & $\mathbf{R}^{2}$ \\
\hline November & -0.086 & 0.053 & 0.043 & - & 0.839 & 0.763 & 0.200 & 0.122 & 0.147 & 208.1 & 0.481 \\
\hline January & 0.093 & 0.040 & 0.054 & 0.021 & 0.766 & 0.979 & 0.243 & 0.139 & 0.152 & 166.6 & 0.464 \\
\hline March & 0.497 & 0.072 & 0.057 & 0.028 & 0.366 & 0.830 & - & 0.138 & 0.177 & 144 & 0.390 \\
\hline May & 0.332 & 0.062 & 0.033 & - & 0.565 & 0.285 & - & 0.122 & 0.154 & 154.8 & 0.364 \\
\hline July & -0.379 & 0.039 & 0.035 & -0.008 & 0.945 & 0.724 & 0.468 & 0.207 & 0.141 & 268.4 & 0.582 \\
\hline September & -0.642 & 0.043 & 0.030 & - & 1.127 & 0.958 & 0.457 & 0.186 & 0.144 & 359.4 & 0.615 \\
\hline
\end{tabular}

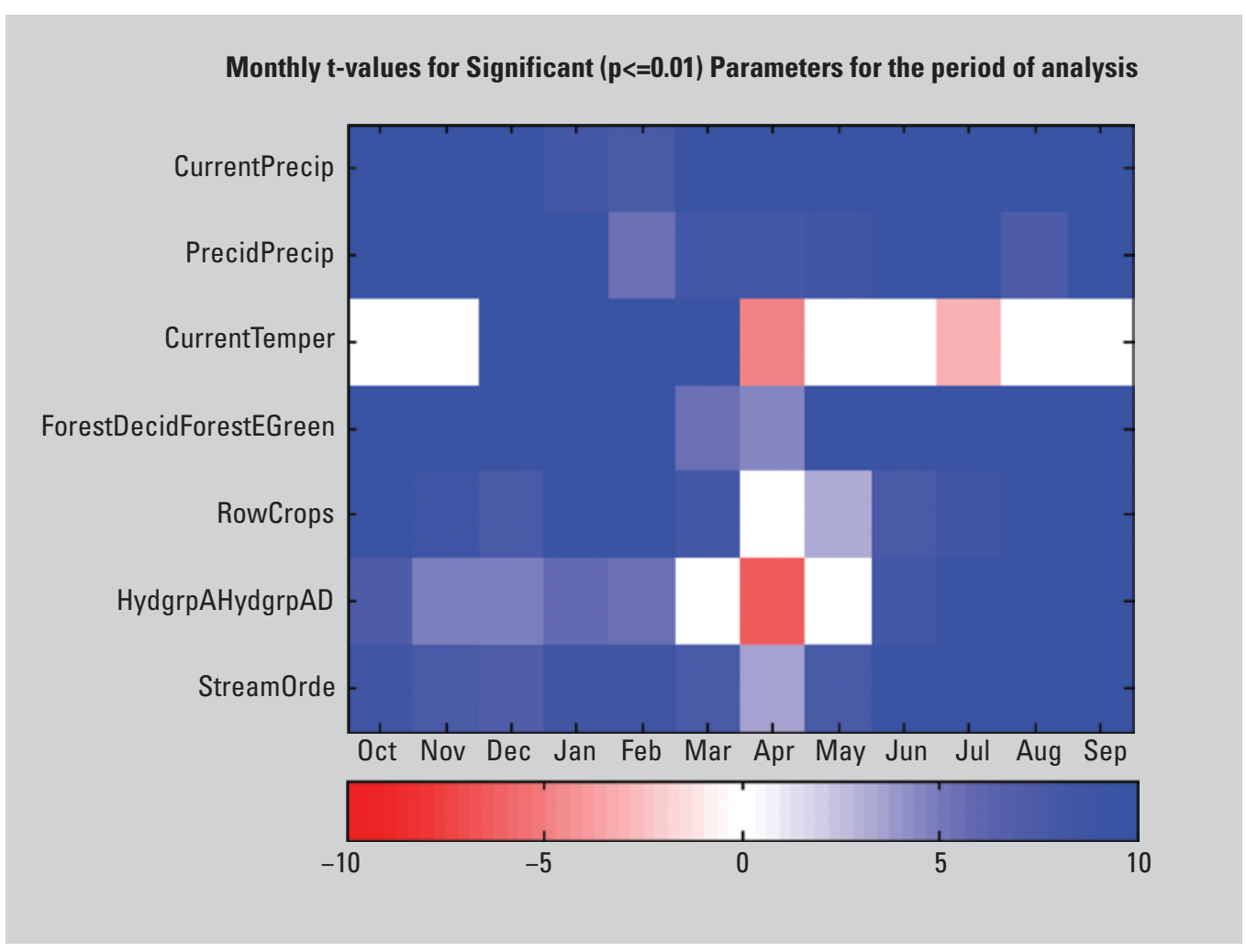

Figure 11. Image showing the t-value-indexed colors corresponding to each explanatory variable in the monthly regression equation for estimating water yield for study area 4 (cataloguing units 04060101, 04060102, 04060103, 04060104, 04060105, 04070003, 04070004, 04070005, 04070006, and 04070007) in the U.S. Great Lakes Basin. 


\section{Study Area 5}

Study area 5 is formed by hydrologic subregions 0408 and 0409 near the central part of the U.S. Great Lakes Basin along the southwestern side of Lake Huron in the Lower Peninsula of Michigan (fig. 12). The number of stations included in the analysis ranged from 21 (2008) to 52 (1966) (table 11). Eleven explanatory variables were selected during the regression analysis with the number of variables specified for each monthly equation ranging from 6 (March) to 10 (August) (table 3). Variables and regression statistics based on the stepwise regression for each month are indicated in table 12 and figure 13.

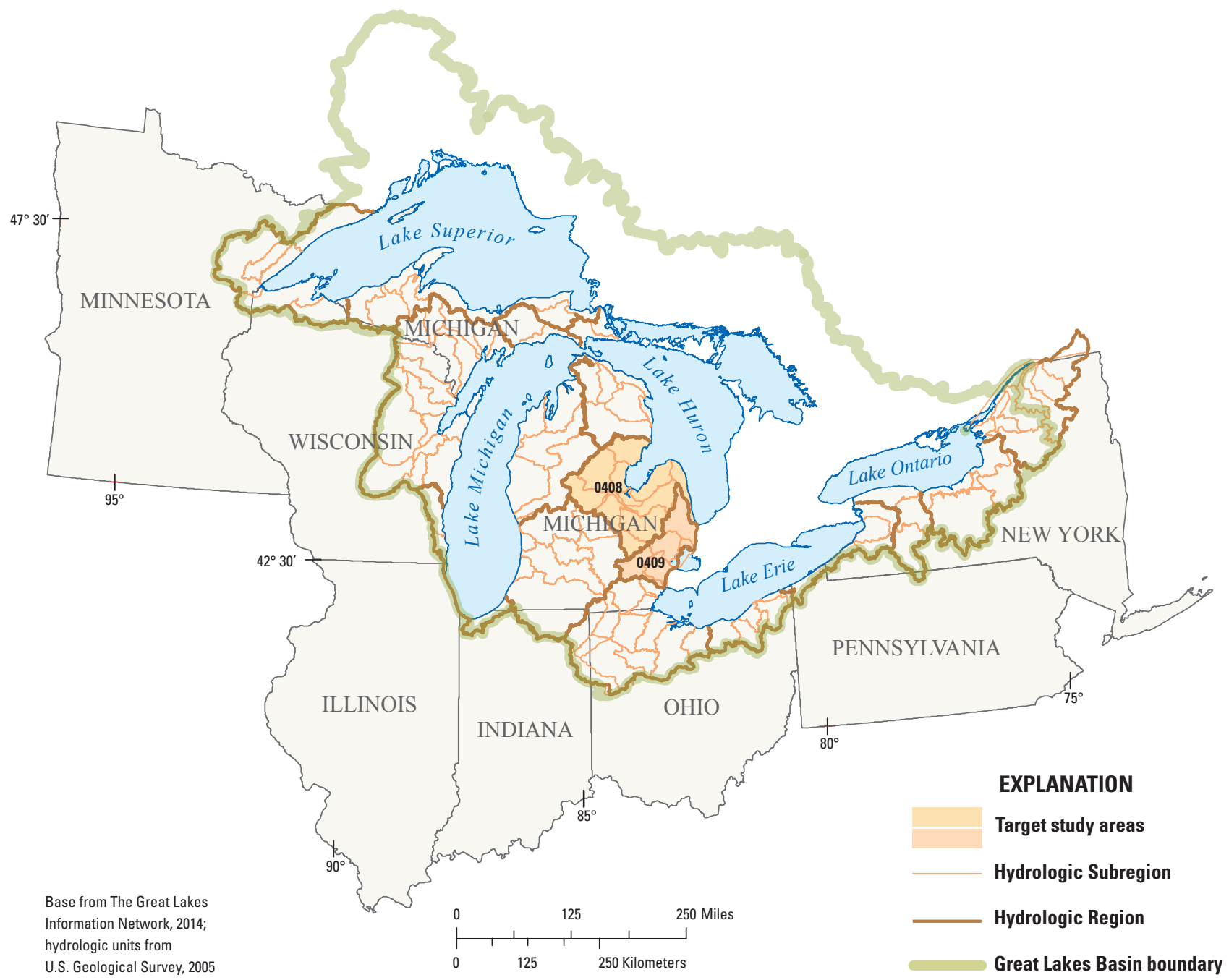

Figure 12. Location of study area 5 (hydrologic subregions 0408 and 0409) in the U.S. Great Lakes Basin. 
Table 11. Streamgaging stations considered for analysis of study area 5 (hydrologic subregions 0408 and 0409 ) in the U.S. Great Lakes Basin, by water year.

$[\bullet$, station active and included in the analysis; $\circ$, station active but not included in the analysis]

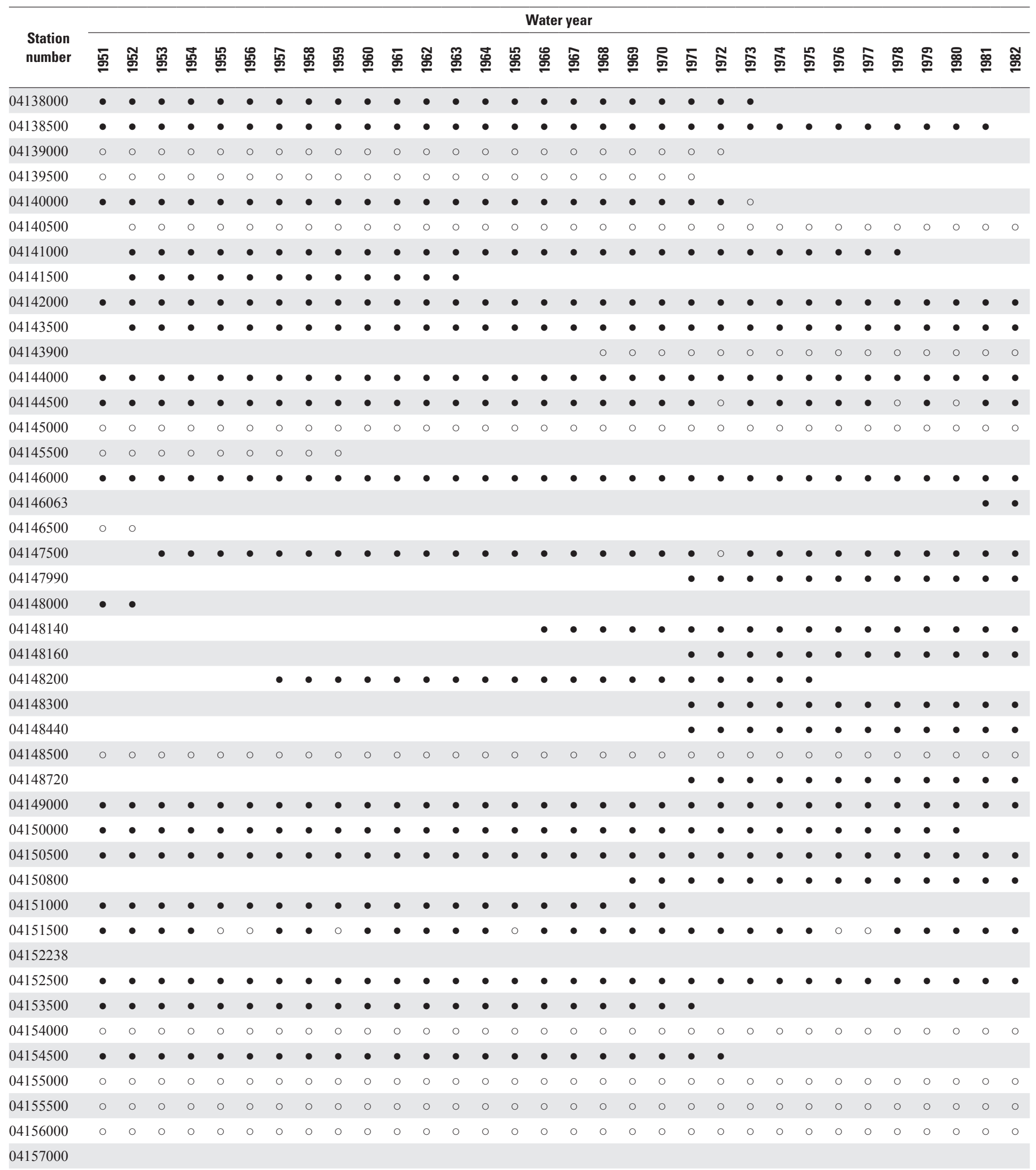


Table 11. Streamgaging stations considered for analysis of study area 5 (hydrologic subregions 0408 and 0409 ) in the U.S. Great Lakes Basin, by water year.-Continued

[ $\bullet$, station active and included in the analysis; ○, station active but not included in the analysis]

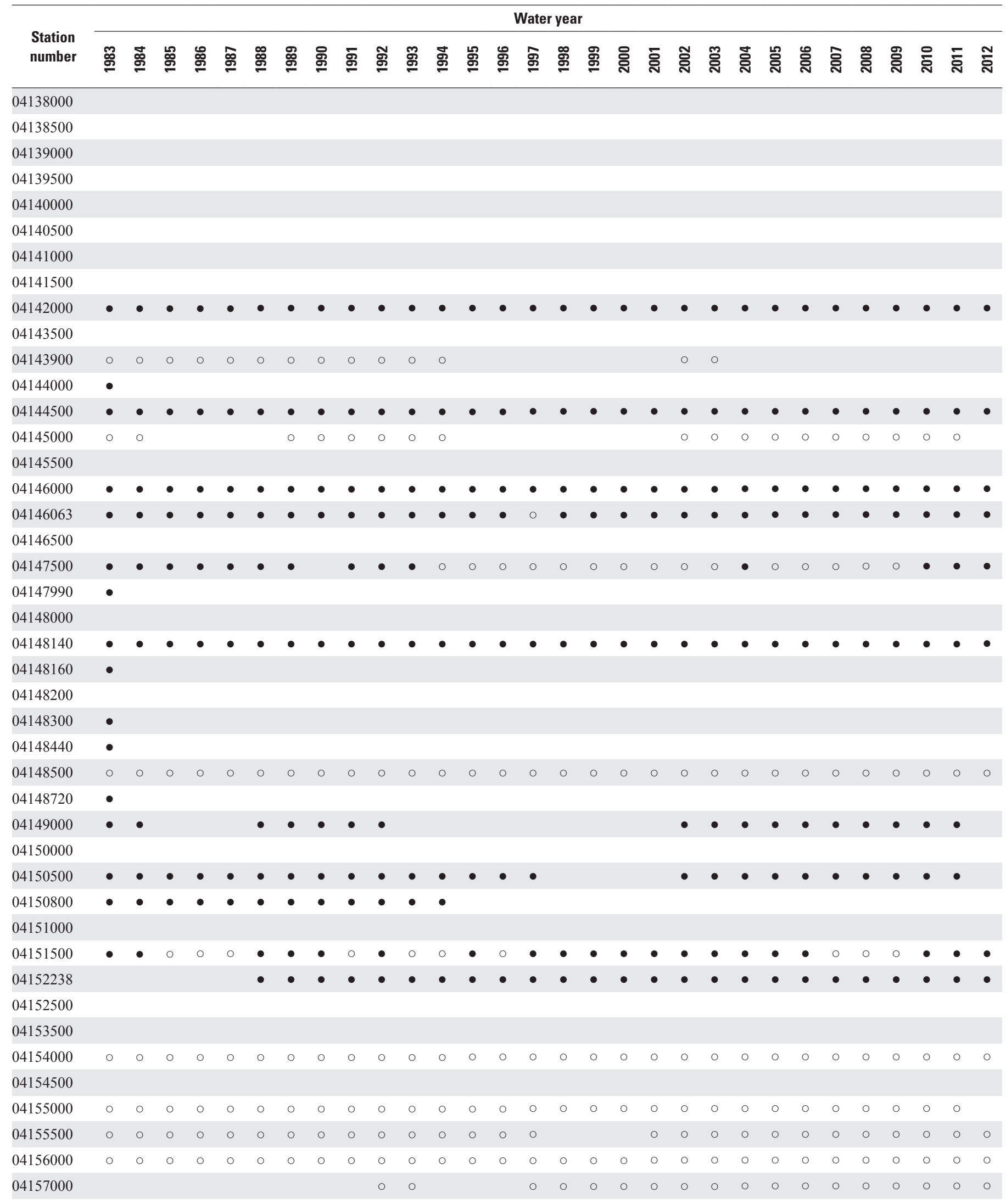


Table 11. Streamgaging stations considered for analysis of study area 5 (hydrologic subregions 0408 and 0409 ) in the U.S. Great Lakes Basin, by water year.-Continued

[ $\bullet$, station active and included in the analysis; ○, station active but not included in the analysis]

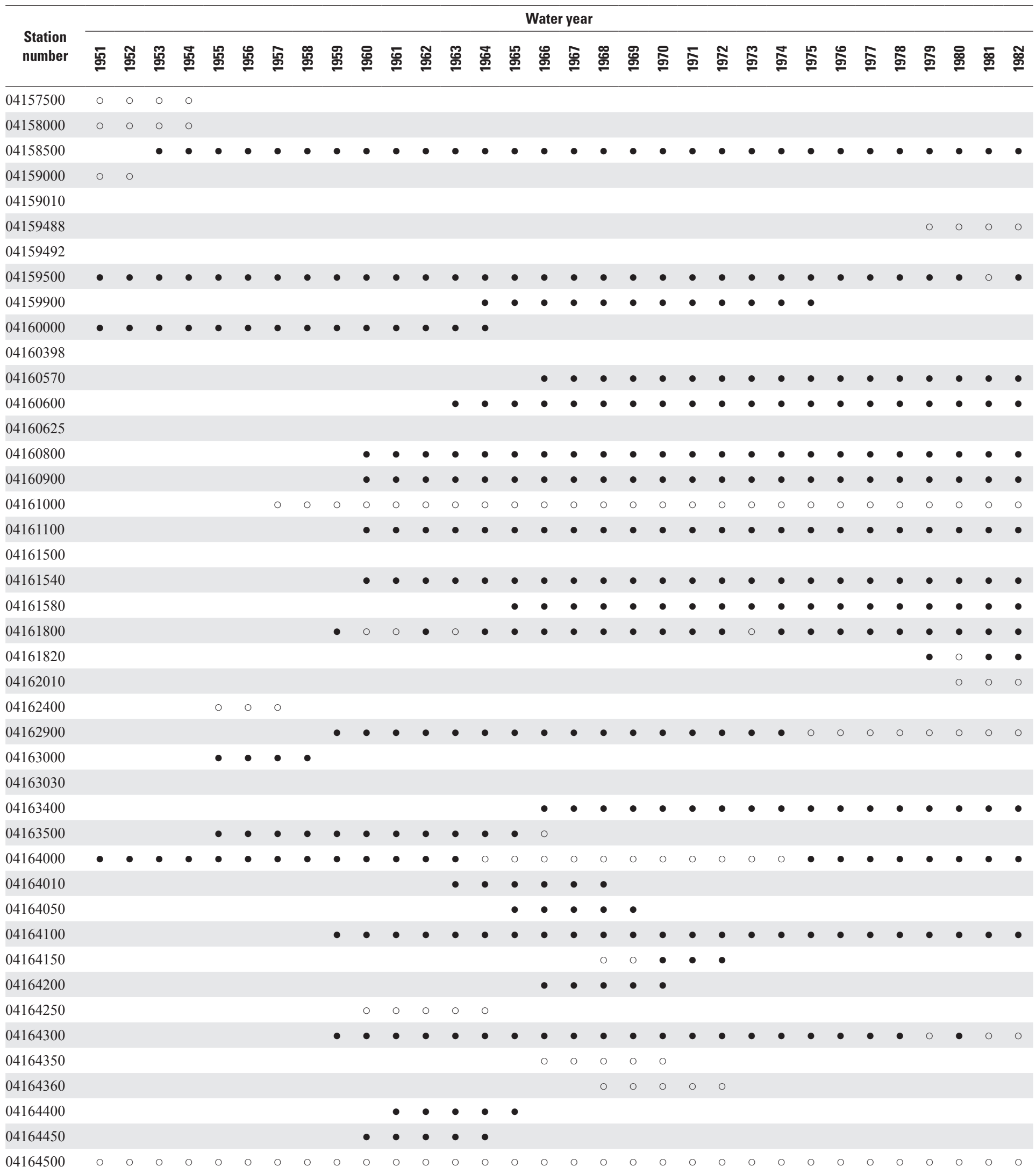


Table 11. Streamgaging stations considered for analysis of study area 5 (hydrologic subregions 0408 and 0409) in the U.S. Great Lakes Basin, by water year.-Continued

[ $\bullet$, station active and included in the analysis; $\odot$, station active but not included in the analysis]

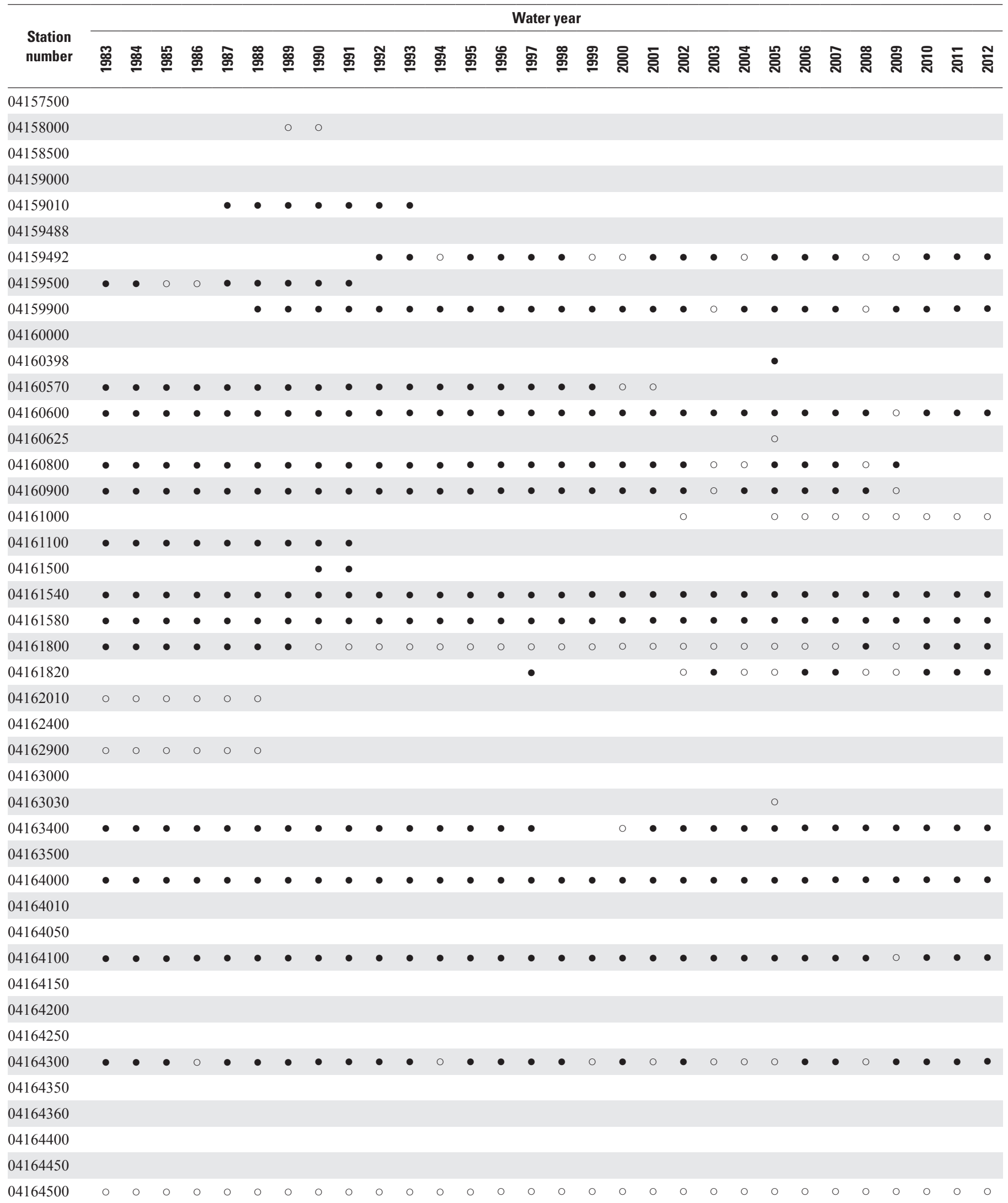


Table 11. Streamgaging stations considered for analysis of study area 5 (hydrologic subregions 0408 and 0409) in the U.S. Great Lakes Basin, by water year.-Continued

$[\bullet$, station active and included in the analysis; $\odot$, station active but not included in the analysis]

\begin{tabular}{|c|c|c|c|c|c|c|c|c|c|c|c|c|c|c|c|c|c|c|c|c|c|c|c|c|c|c|c|c|c|c|c|c|}
\hline \multirow{2}{*}{$\begin{array}{l}\text { Station } \\
\text { number }\end{array}$} & \multicolumn{32}{|c|}{ Water year } \\
\hline & 鸟 & స్్ & ซ్ర్ & 蒙 & 잉 & 냉 & 夸 & 哭 & 哭 & \%్రా & ळ్ & ช్ ซ్ & ஜூ & চ্ّ & 농 & ஜू & ङ & \%్̊ & 灾 & 윰 & $\bar{\sigma}$ & న్ & ๙ூ & 离 & 占 & $\stackrel{\circ}{\frac{0}{\sigma}}$ & Б્ & $\stackrel{\infty}{\sigma}$ & হั & 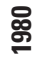 & ळ্口 & \% \\
\hline 04164600 & & & & & & & & & & & & & & & $\bullet$ & $\bullet$ & $\bullet$ & $\bullet$ & $\bullet$ & & & & & & & & & & & & & \\
\hline 04164800 & & & & & & & & & & & & & $\bullet$ & $\bullet$ & $\bullet$ & $\bullet$ & o & 0 & & $\bullet$ & $\bullet$ & $\bullet$ & $\bullet$ & $\bullet$ & $\bullet$ & $\bullet$ & $\bullet$ & $\bullet$ & $\bullet$ & $\bullet$ & $\bullet$ & $\bullet$ \\
\hline \multicolumn{33}{|l|}{04164980} \\
\hline 04165200 & & & & & & & & & & $\bullet$ & $\bullet$ & $\bullet$ & $\bullet$ & $\bullet$ & & & & & & & & & & & & & & & & & & \\
\hline 04165500 & $\bullet$ & $\bullet$ & $\bullet$ & $\bullet$ & $\bullet$ & $\bullet$ & $\bullet$ & $\bullet$ & $\bullet$ & $\bullet$ & $\bullet$ & $\bullet$ & $\circ$ & $\circ$ & $\circ$ & o & ० & $\circ$ & $\circ$ & $\bullet$ & $\circ$ & $\bullet$ & • & • & $\bullet$ & $\bullet$ & $\bullet$ & $\bullet$ & $\bullet$ & $\bullet$ & $\bullet$ & - \\
\hline \multicolumn{33}{|l|}{04165557} \\
\hline \multicolumn{33}{|l|}{04165559} \\
\hline 04166000 & $\bullet$ & $\bullet$ & $\bullet$ & $\bullet$ & $\bullet$ & $\bullet$ & $\bullet$ & $\bullet$ & $\bullet$ & $\bullet$ & $\bullet$ & $\bullet$ & $\bullet$ & $\bullet$ & $\bullet$ & $\bullet$ & $\bullet$ & $\bullet$ & $\bullet$ & $\bullet$ & $\bullet$ & $\bullet$ & $\bullet$ & $\bullet$ & $\bullet$ & $\bullet$ & $\bullet$ & $\bullet$ & $\bullet$ & $\bullet$ & $\bullet$ & $\bullet$ \\
\hline \multicolumn{33}{|l|}{04166040} \\
\hline 04166100 & & & & & & & & & $\bullet$ & $\bullet$ & $\bullet$ & $\bullet$ & $\bullet$ & $\bullet$ & $\bullet$ & $\bullet$ & $\bullet$ & $\bullet$ & $\bullet$ & $\bullet$ & $\bullet$ & $\bullet$ & $\bullet$ & $\bullet$ & $\bullet$ & $\bullet$ & $\bullet$ & $\bullet$ & $\bullet$ & $\bullet$ & $\bullet$ & - \\
\hline 04166200 & & & & & & & & & $\bullet$ & $\bullet$ & $\bullet$ & $\bullet$ & $\bullet$ & $\bullet$ & $\bullet$ & $\bullet$ & $\bullet$ & $\bullet$ & $\bullet$ & $\bullet$ & $\bullet$ & $\bullet$ & $\bullet$ & $\bullet$ & $\bullet$ & $\bullet$ & $\bullet$ & $\bullet$ & $\bullet$ & $\bullet$ & $\bullet$ & $\bullet$ \\
\hline 04166300 & & & & & & & & & $\bullet$ & $\bullet$ & $\bullet$ & $\bullet$ & $\bullet$ & $\bullet$ & $\bullet$ & $\bullet$ & $\bullet$ & $\bullet$ & $\bullet$ & $\bullet$ & $\bullet$ & $\bullet$ & $\bullet$ & $\bullet$ & $\bullet$ & $\bullet$ & $\bullet$ & $\bullet$ & $\bullet$ & $\bullet$ & $\bullet$ & $\bullet$ \\
\hline \multicolumn{33}{|l|}{04166470} \\
\hline 04166500 & $\circ$ & $\circ$ & $\circ$ & $\circ$ & $\circ$ & $\circ$ & $\circ$ & $\circ$ & $\circ$ & $\circ$ & $\circ$ & $\circ$ & $\circ$ & $\circ$ & $\circ$ & $\circ$ & $\circ$ & $\circ$ & $\circ$ & $\circ$ & $\circ$ & $\circ$ & o & $\circ$ & $\circ$ & $\circ$ & $\circ$ & $\circ$ & $\circ$ & $\circ$ & $\circ$ & ○ \\
\hline \multicolumn{33}{|l|}{04166750} \\
\hline 04167000 & $\circ$ & $\circ$ & $\circ$ & $\circ$ & $\circ$ & $\circ$ & $\circ$ & $\circ$ & $\circ$ & $\circ$ & $\circ$ & $\circ$ & $\circ$ & $\circ$ & $\circ$ & $\circ$ & ○ & $\circ$ & $\circ$ & $\circ$ & $\circ$ & $\circ$ & $\circ$ & $\circ$ & $\circ$ & $\circ$ & $\circ$ & & & & & \\
\hline \multicolumn{33}{|l|}{04167150} \\
\hline \multicolumn{33}{|l|}{04167625} \\
\hline 04168000 & $\bullet$ & $\bullet$ & $\bullet$ & $\bullet$ & $\bullet$ & $\bullet$ & $\bullet$ & $\bullet$ & $\bullet$ & $\bullet$ & $\bullet$ & $\bullet$ & $\bullet$ & $\bullet$ & $\bullet$ & $\bullet$ & o & $\bullet$ & $\bullet$ & $\bullet$ & $\circ$ & $\bullet$ & $\bullet$ & $\circ$ & $\bullet$ & $\bullet$ & $\bullet$ & $\bullet$ & $\bullet$ & $\bullet$ & $\bullet$ & - \\
\hline \multicolumn{33}{|l|}{04168400} \\
\hline \multicolumn{33}{|l|}{04168530} \\
\hline \multicolumn{33}{|l|}{04168580} \\
\hline 04169500 & $\bullet$ & $\bullet$ & $\bullet$ & $\bullet$ & $\bullet$ & $\bullet$ & $\bullet$ & $\bullet$ & $\bullet$ & $\bullet$ & $\bullet$ & $\bullet$ & $\bullet$ & $\bullet$ & $\bullet$ & $\bullet$ & $\bullet$ & $\bullet$ & $\bullet$ & $\bullet$ & $\bullet$ & $\bullet$ & $\bullet$ & $\bullet$ & $\bullet$ & & & & & & & \\
\hline 04170000 & $\bullet$ & $\bullet$ & $\bullet$ & $\bullet$ & $\bullet$ & $\bullet$ & $\bullet$ & $\bullet$ & $\bullet$ & $\bullet$ & $\bullet$ & $\bullet$ & $\bullet$ & $\bullet$ & $\bullet$ & $\bullet$ & $\bullet$ & $\bullet$ & $\bullet$ & $\bullet$ & $\bullet$ & $\bullet$ & $\bullet$ & $\bullet$ & $\bullet$ & $\bullet$ & $\bullet$ & $\bullet$ & $\circ$ & $\bullet$ & $\bullet$ & $\bullet$ \\
\hline 04170500 & $\circ$ & $\circ$ & $\circ$ & $\circ$ & $\circ$ & $\circ$ & $\circ$ & $\circ$ & $\circ$ & $\circ$ & $\circ$ & $\circ$ & $\circ$ & $\circ$ & $\circ$ & $\circ$ & $\circ$ & $\circ$ & $\circ$ & $\circ$ & $\circ$ & $\circ$ & $\circ$ & $\circ$ & $\circ$ & $\circ$ & $\circ$ & o & $\circ$ & $\circ$ & $\circ$ & ० \\
\hline 04171500 & & $\bullet$ & $\bullet$ & $\bullet$ & $\bullet$ & $\bullet$ & $\bullet$ & $\bullet$ & $\bullet$ & $\bullet$ & $\bullet$ & $\bullet$ & $\bullet$ & $\bullet$ & $\bullet$ & $\bullet$ & $\bullet$ & $\bullet$ & & & & & & & & & & & & & & \\
\hline 04172000 & & $\bullet$ & $\bullet$ & $\bullet$ & $\bullet$ & $\bullet$ & $\bullet$ & $\bullet$ & $\bullet$ & $\bullet$ & $\bullet$ & $\bullet$ & $\bullet$ & $\bullet$ & $\bullet$ & $\bullet$ & $\bullet$ & $\bullet$ & $\bullet$ & $\bullet$ & $\bullet$ & $\bullet$ & $\bullet$ & $\bullet$ & $\bullet$ & $\bullet$ & $\bullet$ & $\bullet$ & $\circ$ & $\bullet$ & $\bullet$ & $\bullet$ \\
\hline 04172500 & $\bullet$ & $\bullet$ & $\bullet$ & $\bullet$ & $\bullet$ & $\bullet$ & $\bullet$ & $\bullet$ & $\bullet$ & $\bullet$ & $\bullet$ & $\bullet$ & $\bullet$ & $\bullet$ & $\bullet$ & $\bullet$ & $\bullet$ & $\bullet$ & $\bullet$ & $\bullet$ & $\bullet$ & & & & & & & & & & & \\
\hline 04173000 & $\bullet$ & $\bullet$ & $\bullet$ & $\bullet$ & $\bullet$ & $\bullet$ & $\circ$ & $\bullet$ & $\bullet$ & $\bullet$ & $\bullet$ & $\bullet$ & $\bullet$ & $\circ$ & $\circ$ & $\bullet$ & o & $\bullet$ & $\circ$ & $\bullet$ & $\circ$ & $\bullet$ & & & & $\bullet$ & $\bullet$ & & & & & \\
\hline 04173500 & & & $\bullet$ & $\bullet$ & $\bullet$ & $\bullet$ & $\bullet$ & $\bullet$ & $\bullet$ & $\bullet$ & $\bullet$ & $\bullet$ & $\bullet$ & $\bullet$ & $\bullet$ & $\bullet$ & $\bullet$ & $\bullet$ & $\bullet$ & $\bullet$ & $\bullet$ & $\bullet$ & $\bullet$ & $\bullet$ & $\bullet$ & $\bullet$ & $\bullet$ & $\bullet$ & $\circ$ & $\bullet$ & $\bullet$ & $\bullet$ \\
\hline 04174500 & $\circ$ & $\circ$ & $\circ$ & $\circ$ & $\circ$ & $\circ$ & $\circ$ & $\circ$ & $\circ$ & $\circ$ & $\circ$ & $\circ$ & $\circ$ & $\circ$ & $\circ$ & $\circ$ & $\circ$ & $\circ$ & $\circ$ & $\circ$ & $\circ$ & $\circ$ & $\circ$ & $\circ$ & $\circ$ & $\circ$ & $\circ$ & $\circ$ & $\circ$ & $\circ$ & $\circ$ & ० \\
\hline \multicolumn{33}{|l|}{04174518} \\
\hline 04174800 & & & & & & & & & & & & & & & & & & & & & & & & & o & o & o & o & $\circ$ & $\circ$ & $\circ$ & o \\
\hline
\end{tabular}


Table 11. Streamgaging stations considered for analysis of study area 5 (hydrologic subregions 0408 and 0409) in the U.S. Great Lakes Basin, by water year.-Continued

[ $\bullet$, station active and included in the analysis; $\circ$, station active but not included in the analysis]

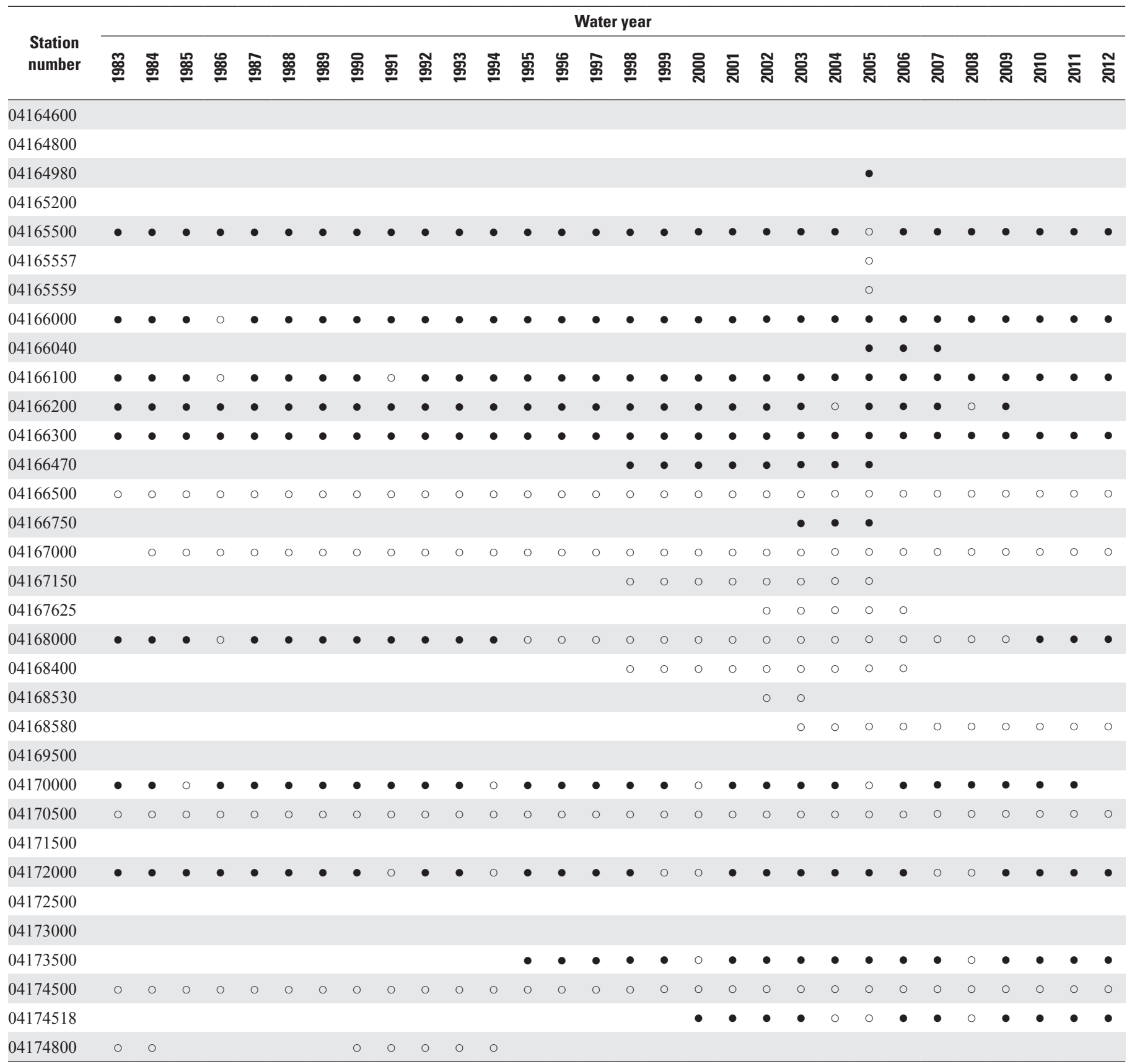


Table 12. Regression statistics and explanatory variables used in the AFINCH (Analysis of Flows In Networks of CHannels) analysis for study area 5 (hydrologic subregions 0408 and 0409) in the U.S. Great Lakes Basin, by month.

[-, indicates variable not included in monthly regression equation; GDD, growing degree days; RMSE, root mean square error; $\mathrm{R}^{2}$, coefficient of determination. See appendix tables for variable descriptions]

\begin{tabular}{|c|c|c|c|c|c|c|c|c|c|c|c|c|c|c|c|}
\hline \multirow[b]{2}{*}{ Month } & \multicolumn{12}{|c|}{ Regression coefficients } & \multicolumn{3}{|c|}{ Regression fit statistics } \\
\hline & Intercept & $\begin{array}{c}\text { Current } \\
\text { precipita- } \\
\text { tion }\end{array}$ & $\begin{array}{l}\text { Preceding } \\
\text { precipita- } \\
\text { tion }\end{array}$ & $\begin{array}{c}\text { Current } \\
\text { tempera- } \\
\text { ture }\end{array}$ & $\begin{array}{l}\text { Pasture } \\
\text { hay }\end{array}$ & $\begin{array}{l}\text { Row } \\
\text { crops }\end{array}$ & $\begin{array}{c}\text { Grass } \\
\text { herbaceous }\end{array}$ & $\begin{array}{c}\text { Stream } \\
\text { order }\end{array}$ & $\begin{array}{l}\text { Mean } \\
\text { slope }\end{array}$ & $\begin{array}{c}\text { Hydric } \\
\text { group B } \\
\text { and BD }\end{array}$ & $\begin{array}{c}\text { Coarse } \\
\text { outwash }\end{array}$ & $\begin{array}{c}\text { Average } \\
\text { GDD }\end{array}$ & RMSE & F-statistic & $\mathbf{R}^{2}$ \\
\hline November & 0.0815 & 0.1212 & 0.0795 & -0.0137 & -0.5761 & -0.1675 & - & 0.1342 & 0.1100 & -0.1647 & - & - & 0.203 & 292.3 & 0.512 \\
\hline January & 0.1645 & 0.1383 & 0.1078 & 0.0443 & -0.5149 & 0.1856 & 1.5386 & 0.2060 & 0.1442 & -0.2622 & - & - & 0.232 & 280.1 & 0.530 \\
\hline March & 1.0156 & 0.1500 & 0.1153 & - & -0.2659 & 0.3633 & - & - & - & - & -0.1671 & $-9.5 \mathrm{E}-05$ & 0.284 & 174.3 & 0.319 \\
\hline May & 0.2966 & 0.1606 & 0.0782 & -0.0242 & -0.7910 & - & 2.5436 & 0.1041 & 0.0765 & - & -0.1402 & 4.0E-05 & 0.184 & 515.9 & 0.675 \\
\hline July & -0.3059 & 0.0998 & 0.0745 & - & -1.0679 & - & 2.3501 & 0.1598 & 0.1173 & -0.2079 & -0.1211 & $4.8 \mathrm{E}-05$ & 0.186 & 286 & 0.536 \\
\hline
\end{tabular}

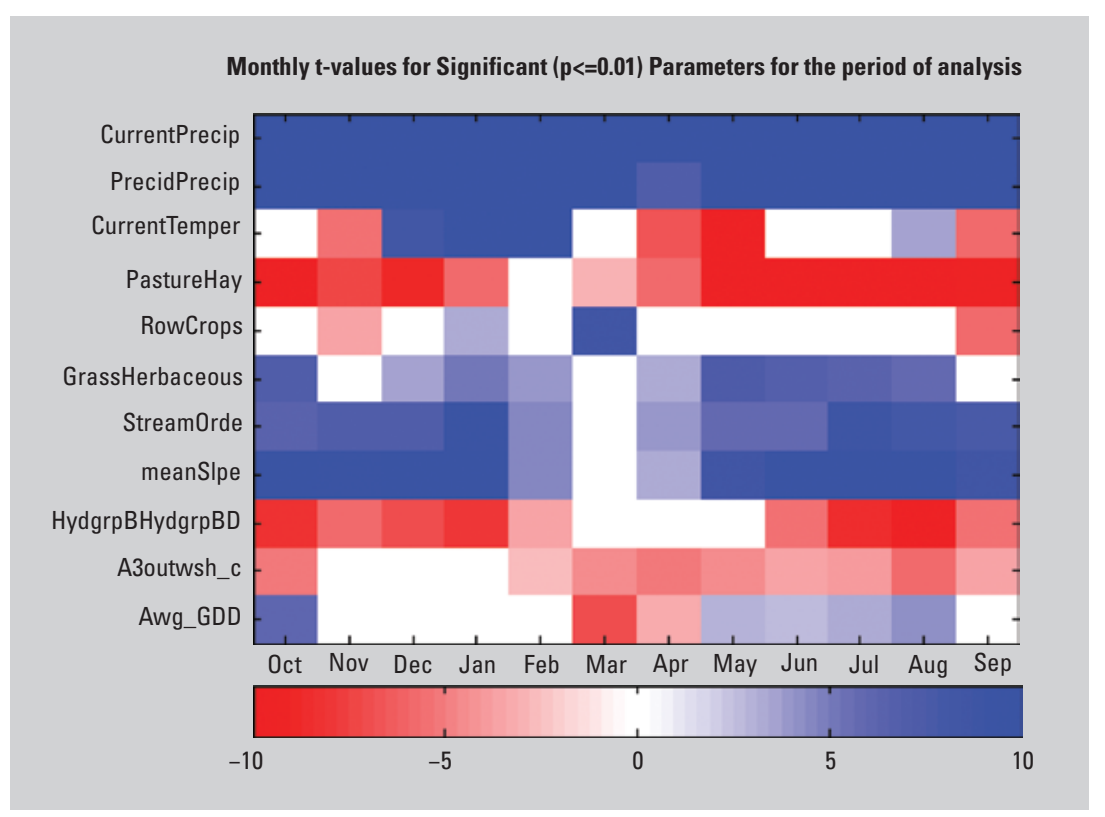

Figure 13. Image showing the t-value-indexed colors corresponding to each explanatory variable in the monthly regression equation for estimating water yield for study area 5 (hydrologic subregions 0408 and 0409) in the U.S. Great Lakes Basin. 


\section{Study Area 6}

Study area 6 is formed by hydrologic subregions 0410 and 0411 in the southeastern part of the U.S. Great Lakes Basin and encompasses parts of Michigan, Ohio, Indiana, and a small part of Pennsylvania (fig. 14). The number of stations included in the analysis ranged from $26(1953,1963$, 1982, and 1983) to 48 (2002) (table 13). Eleven explanatory variables were selected during the regression analysis with the number of variables specified for each monthly equation ranging from 7 (July) to 11 (April and September) (table 3). Variables and regression statistics based on the stepwise regression for each month are indicated in table 14 and figure 15 .

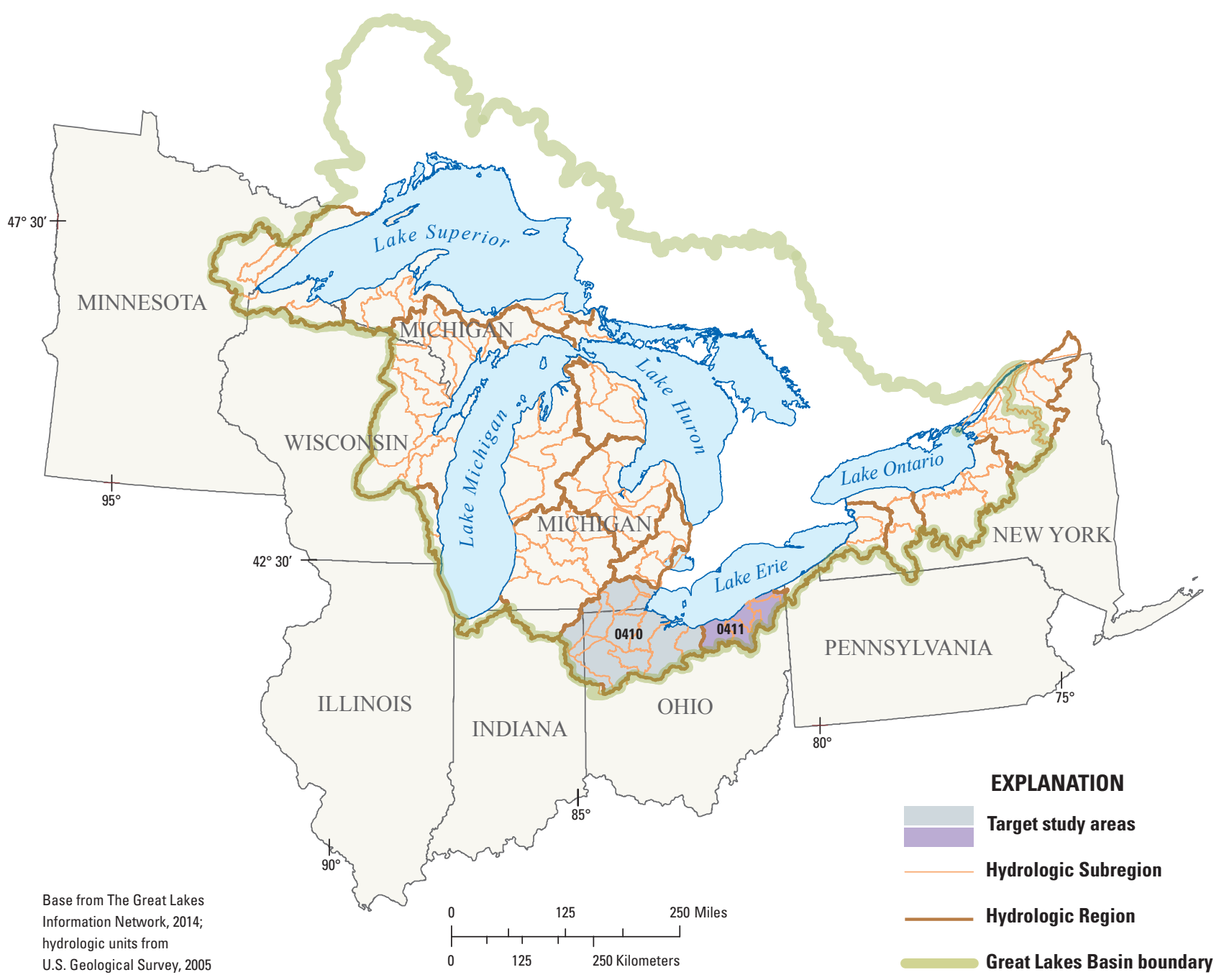

Figure 14. Location of study area 6 (hydrologic subregions 0410 and 0411) in the U.S. Great Lakes Basin. 
Table 13. Streamgaging stations considered for analysis of study area 6 (hydrologic subregions 0410 and 0411 ) in the U.S. Great Lakes Basin, by water year.

$[\bullet$, station active and included in the analysis; $\circ$, station active but not included in the analysis]

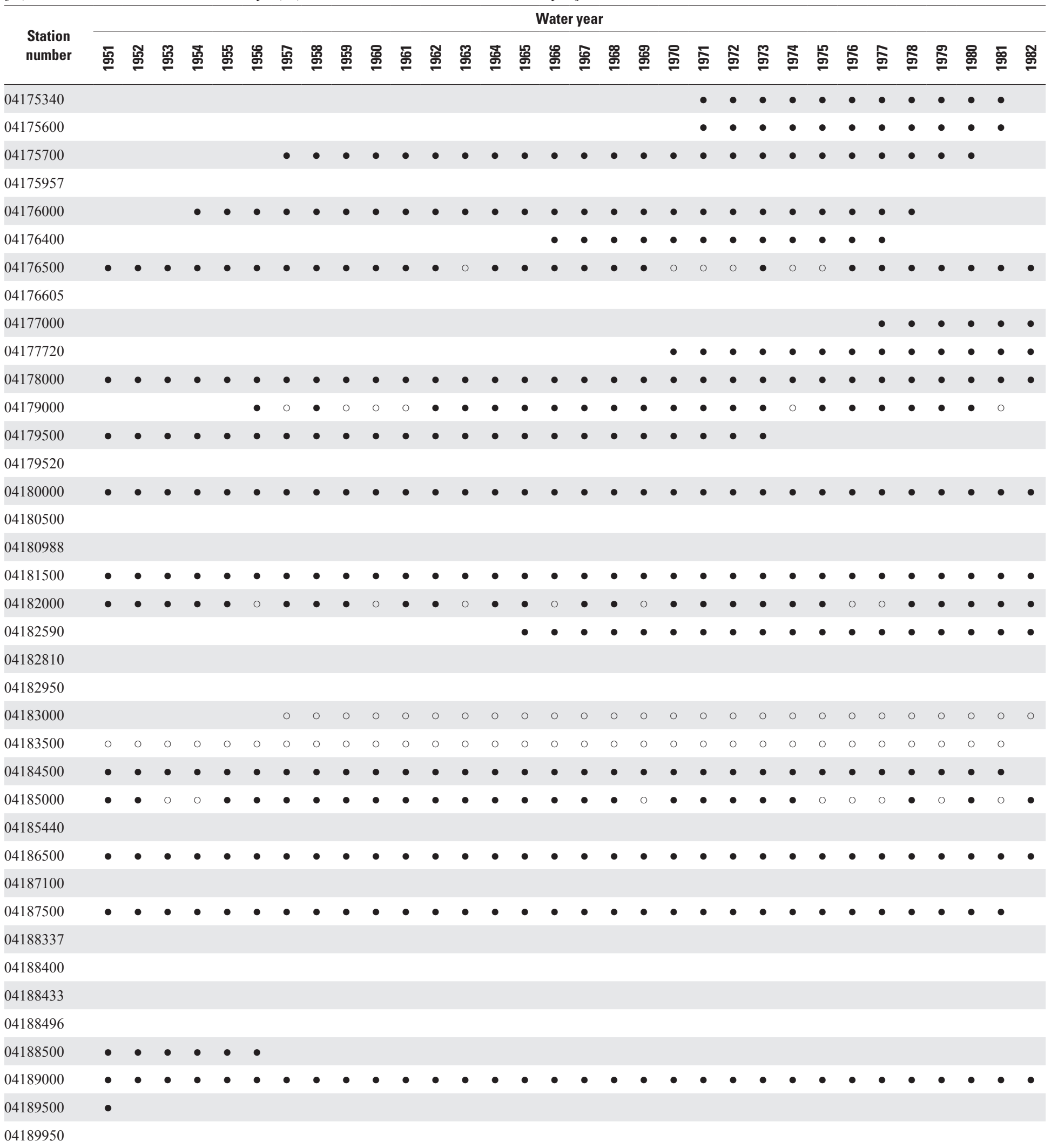

04191000 
Table 13. Streamgaging stations considered for analysis of study area 6 (hydrologic subregions 0410 and 0411 ) in the U.S. Great Lakes Basin, by water year.-Continued

[ $\bullet$, station active and included in the analysis; $\circ$, station active but not included in the analysis]

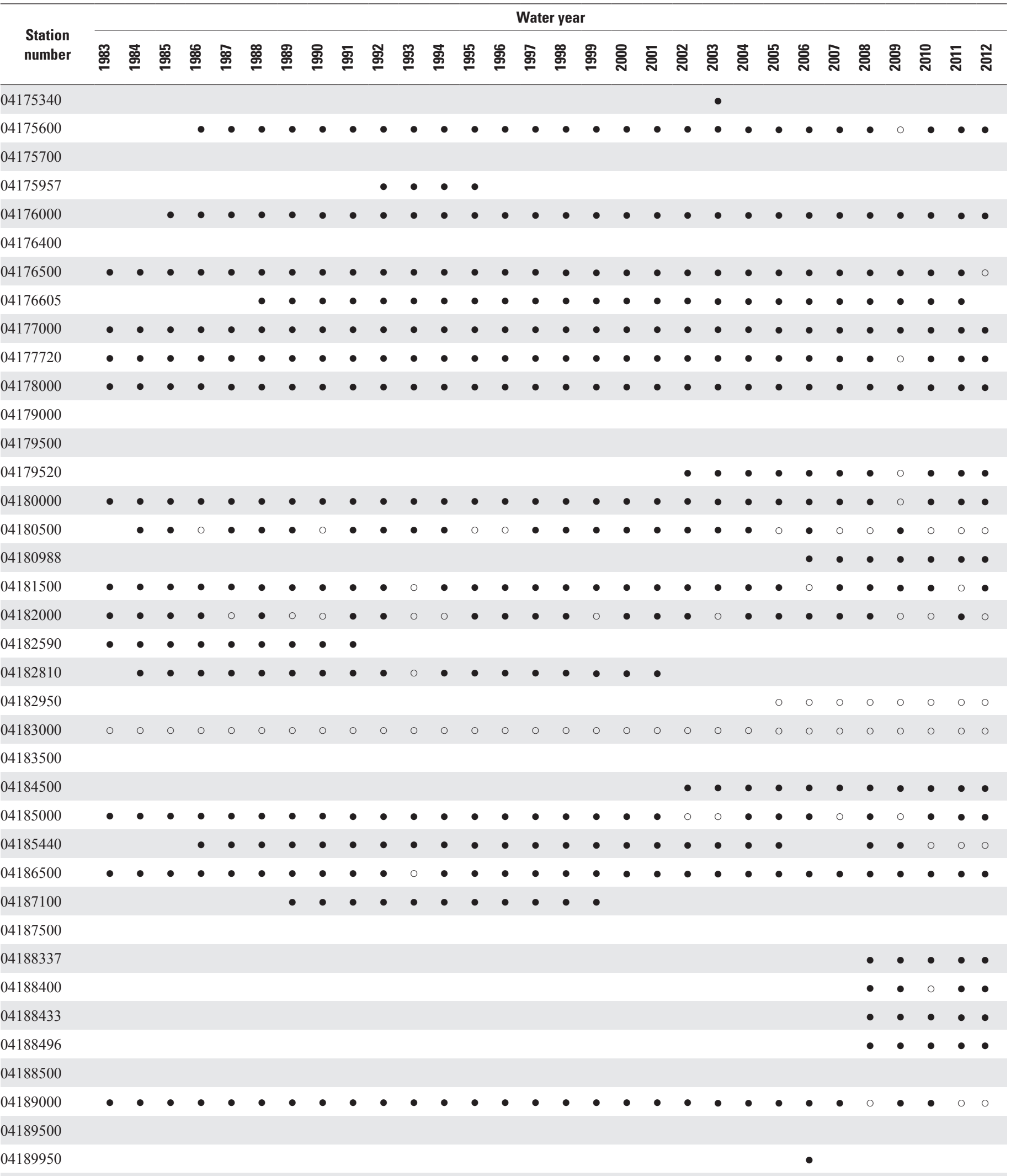

04191000 
Table 13. Streamgaging stations considered for analysis of study area 6 (hydrologic subregions 0410 and 0411 ) in the U.S. Great Lakes Basin, by water year.-Continued

$[\bullet$, station active and included in the analysis; $\odot$, station active but not included in the analysis]

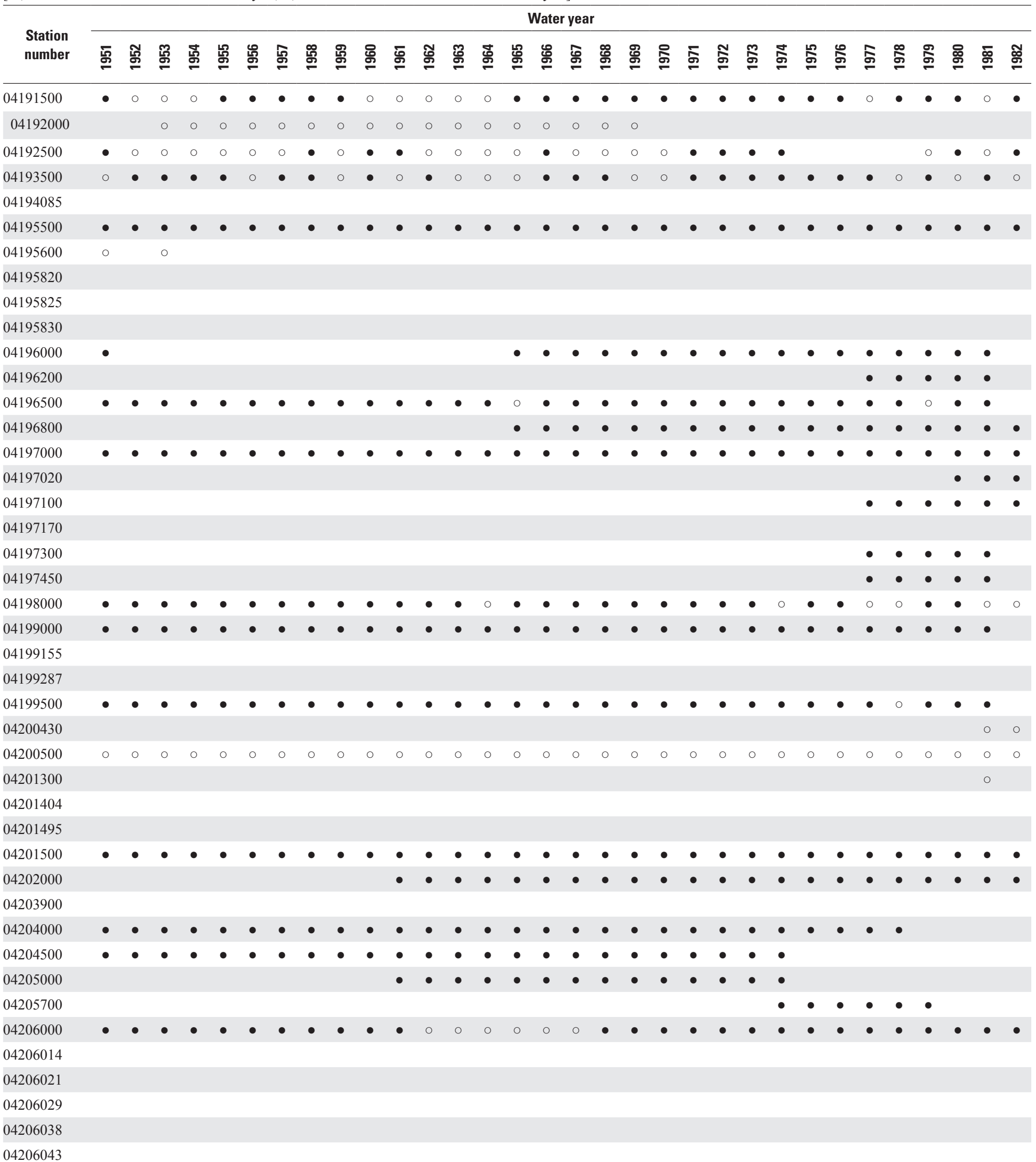

04206043 
Table 13. Streamgaging stations considered for analysis of study area 6 (hydrologic subregions 0410 and 0411 ) in the U.S. Great Lakes Basin, by water year.-Continued

[ $\bullet$, station active and included in the analysis; $\circ$, station active but not included in the analysis]

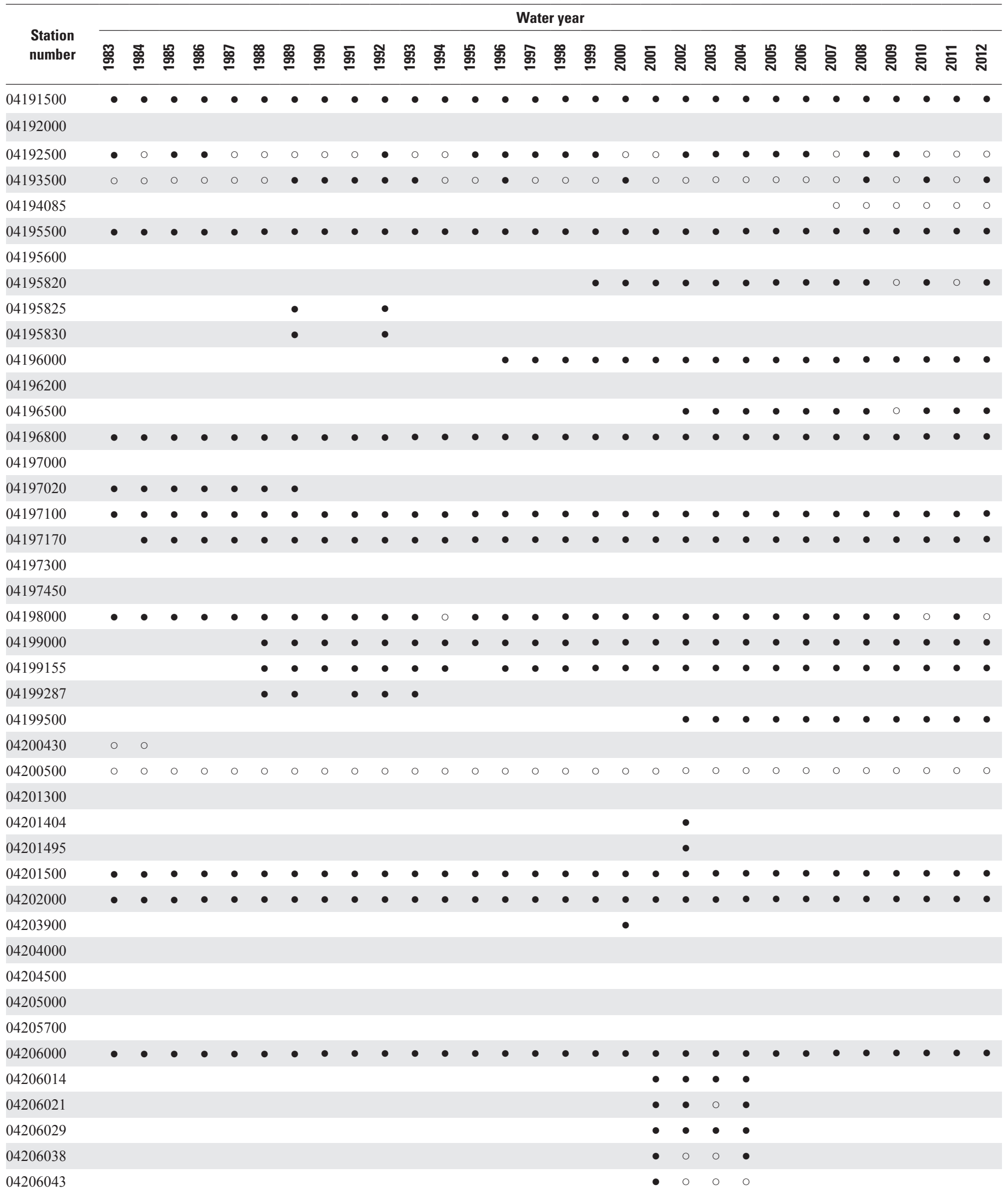


Table 13. Streamgaging stations considered for analysis of study area 6 (hydrologic subregions 0410 and 0411 ) in the U.S. Great Lakes Basin, by water year.-Continued

[ $\bullet$, station active and included in the analysis; $\circ$, station active but not included in the analysis]

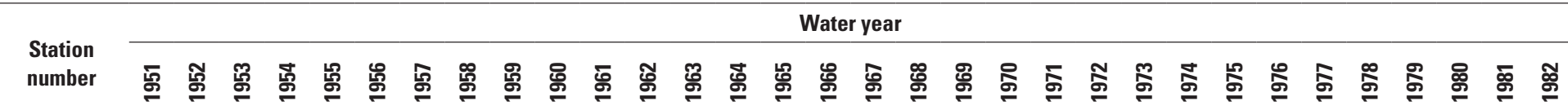

04206208

04206210

04206211

04206212

04206215

04206220

04206250

04206420

04206448

04207016

04207200

04207500

04208000

04208460

04208501

04208502

04208504

04208690

04209000

04210000

04211000

04211500

04211820

04212000

04212100
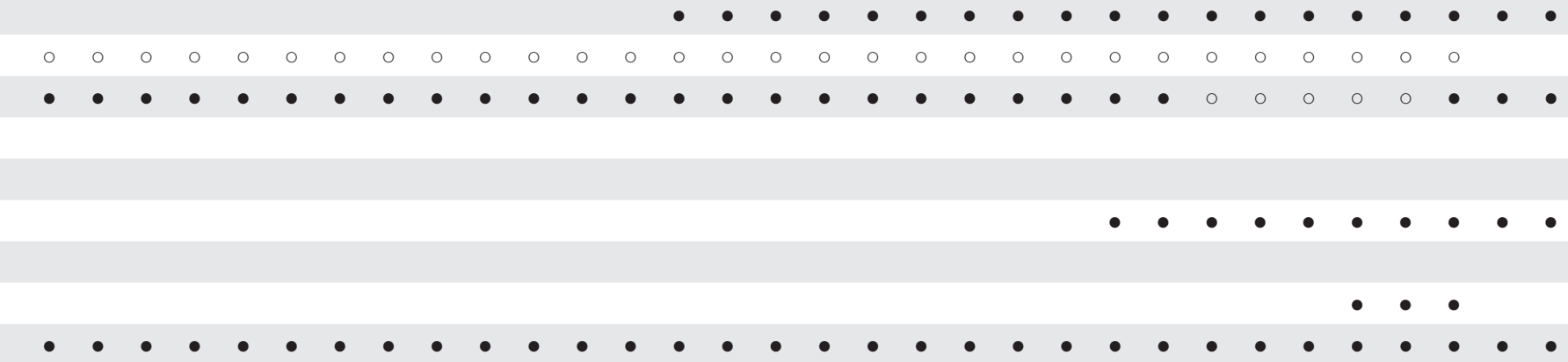
Table 13. Streamgaging stations considered for analysis of study area 6 (hydrologic subregions 0410 and 0411 ) in the U.S. Great Lakes Basin, by water year.-Continued

$[\bullet$, station active and included in the analysis; $\odot$, station active but not included in the analysis]

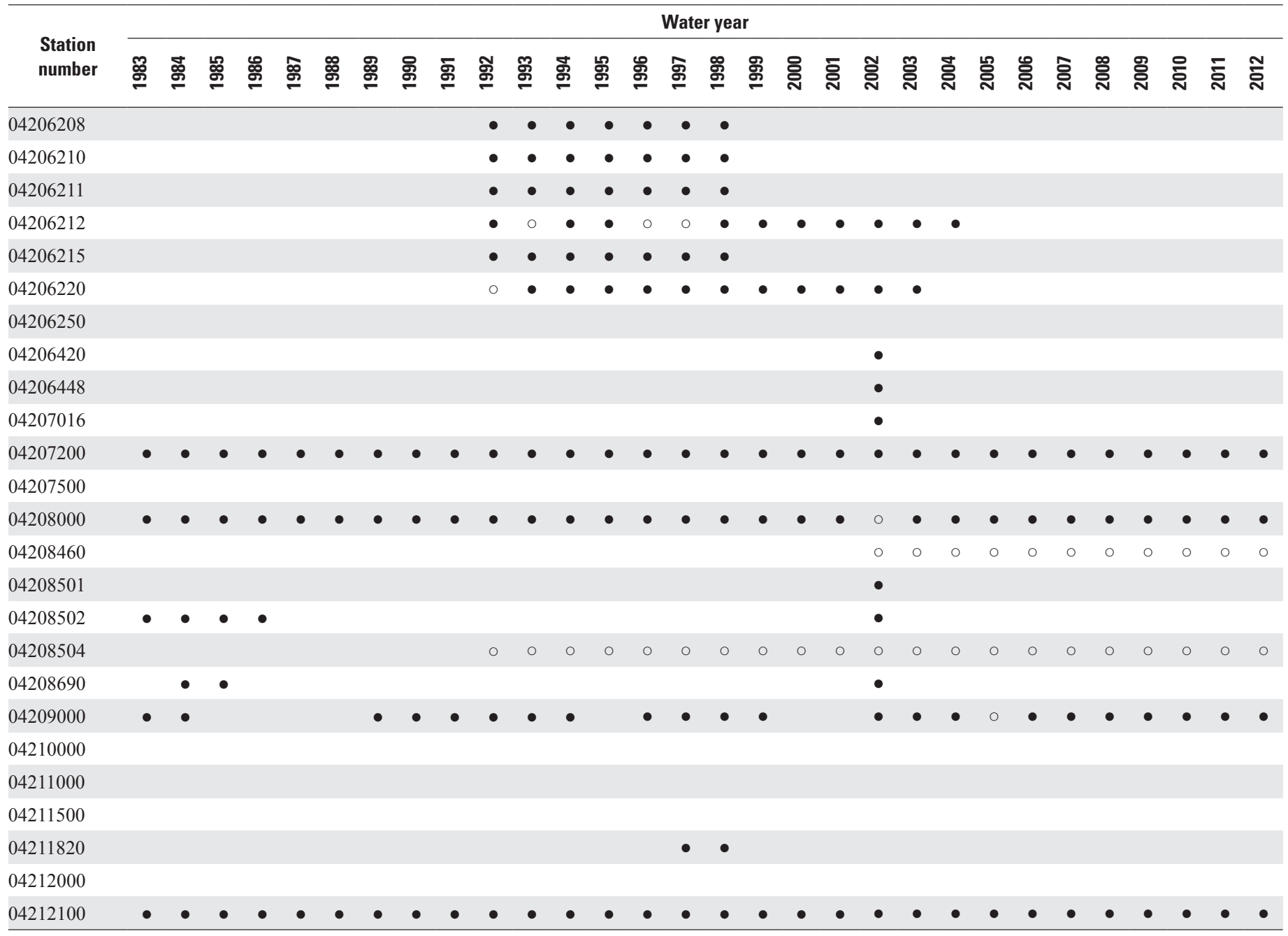


Table 14. Regression statistics and explanatory variables used in the AFINCH (Analysis of Flows In Networks of CHannels) analysis for study area 6 (hydrologic subregions 0410 and 0411 ) in the U.S. Great Lakes Basin, by month.

[-, indicates variable not included in monthly regression equation; RMSE, root mean square error; $\mathrm{R}^{2}$, coefficient of determination. See appendix tables for variable descriptions]

\begin{tabular}{|c|c|c|c|c|c|c|c|c|c|c|c|c|c|c|c|}
\hline \multirow[b]{2}{*}{ Month } & \multicolumn{12}{|c|}{ Regression coefficients } & \multicolumn{3}{|c|}{ Regression fit statistics } \\
\hline & Intercept & $\begin{array}{l}\text { Current } \\
\text { precipi- } \\
\text { tation }\end{array}$ & $\begin{array}{l}\text { Preceding } \\
\text { precipita- } \\
\text { tion }\end{array}$ & $\begin{array}{c}\text { Current } \\
\text { tempera- } \\
\text { ture }\end{array}$ & $\begin{array}{l}\text { Pasture } \\
\text { hay and } \\
\text { row crops }\end{array}$ & Forest & $\begin{array}{l}\text { Fine end } \\
\text { and ground } \\
\text { moraines }\end{array}$ & $\begin{array}{l}\text { Medium end } \\
\text { and ground } \\
\text { moraines }\end{array}$ & $\begin{array}{c}\text { Coarse } \\
\text { outwash and } \\
\text { ice conract }\end{array}$ & $\begin{array}{c}\text { Stream } \\
\text { order }\end{array}$ & $\begin{array}{c}\text { Mean } \\
\text { elevation }\end{array}$ & Mean slope & RMSE & F-statistic & $\mathbf{R}^{2}$ \\
\hline November & 0.421 & 0.226 & 0.090 & -0.015 & -0.355 & -0.063 & 0.076 & 0.259 & - & - & $-1.5 \mathrm{E}-05$ & 0.074 & 0.267 & 516.5 & 0.661 \\
\hline January & 0.495 & 0.288 & 0.081 & 0.037 & - & 0.406 & 0.128 & 0.448 & - & 0.084 & $-1.9 \mathrm{E}-05$ & 0.075 & 0.310 & 442.9 & 0.653 \\
\hline March & 0.930 & 0.254 & 0.047 & -0.035 & - & 0.677 & 0.177 & 0.527 & - & 0.095 & $-2.3 \mathrm{E}-05$ & 0.047 & 0.335 & 224.9 & 0.488 \\
\hline May & 0.985 & 0.200 & 0.072 & -0.018 & -0.218 & - & - & 0.263 & 0.105 & - & $-2.4 \mathrm{E}-05$ & 0.104 & 0.244 & 528.7 & 0.666 \\
\hline July & 0.029 & 0.160 & 0.079 & - & -0.406 & -0.668 & - & - & - & 0.077 & $-7.0 \mathrm{E}-06$ & 0.106 & 0.246 & 478.4 & 0.612 \\
\hline September & 0.338 & 0.141 & 0.077 & -0.011 & -0.521 & -0.601 & 0.113 & 0.156 & 0.339 & 0.099 & $-1.2 \mathrm{E}-05$ & 0.080 & 0.218 & 364 & 0.654 \\
\hline
\end{tabular}

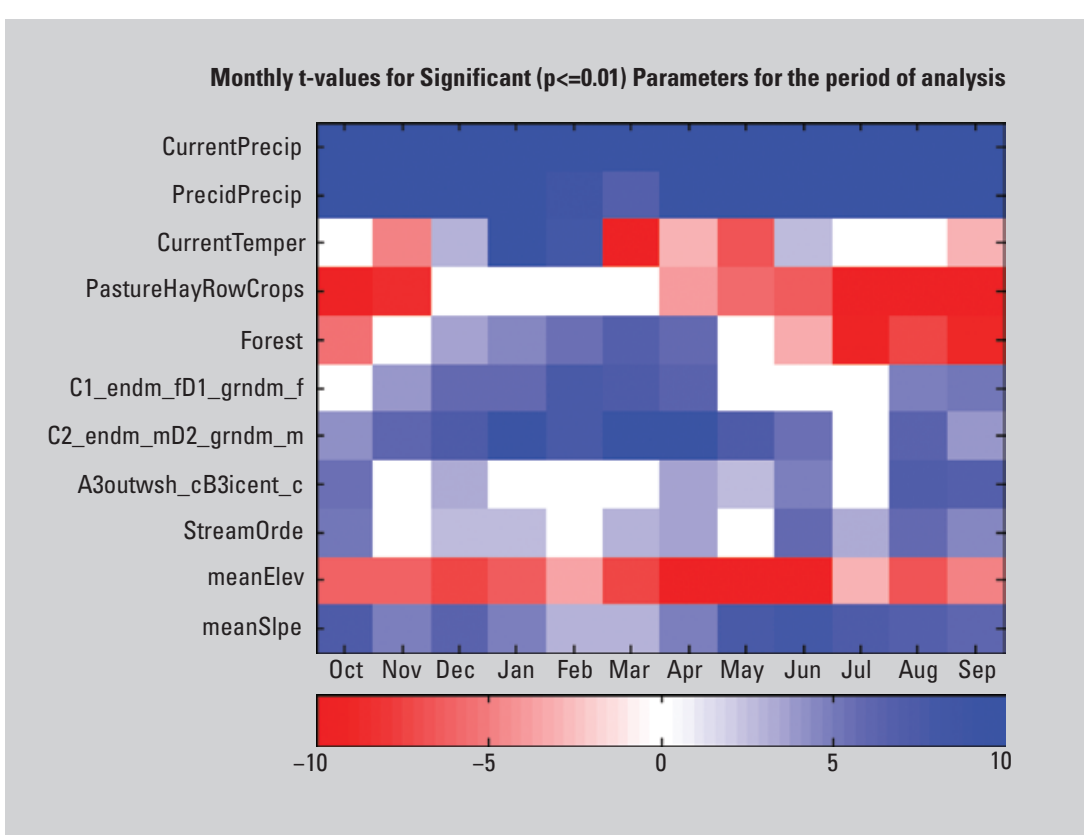

Figure 15. Image showing the t-value-indexed colors corresponding to each explanatory variable in the monthly regression equation for estimating water yield for study area 6 (hydrologic subregions 0410 and 0411) in the U.S. Great Lakes Basin. 


\section{Study Area 7}

Study area 7 is formed by hydrologic subregions 0412 , 0413, 0414, and 0415 in the eastern part of the U.S. Great Lakes Basin and encompasses parts of Pennsylvania, New York, and a small part of Ohio (fig. 16). The number of stations included in the analysis ranged from 31 (1951 and $1953)$ to 65 (1967) (table 15). Twelve explanatory variables were selected during the regression analysis with the number of variables specified for each monthly equation ranging from 6 (February) to 11 (March) (table 3). Variables and regression statistics based on the stepwise regression for each month are indicated in table 16 and figure 17.

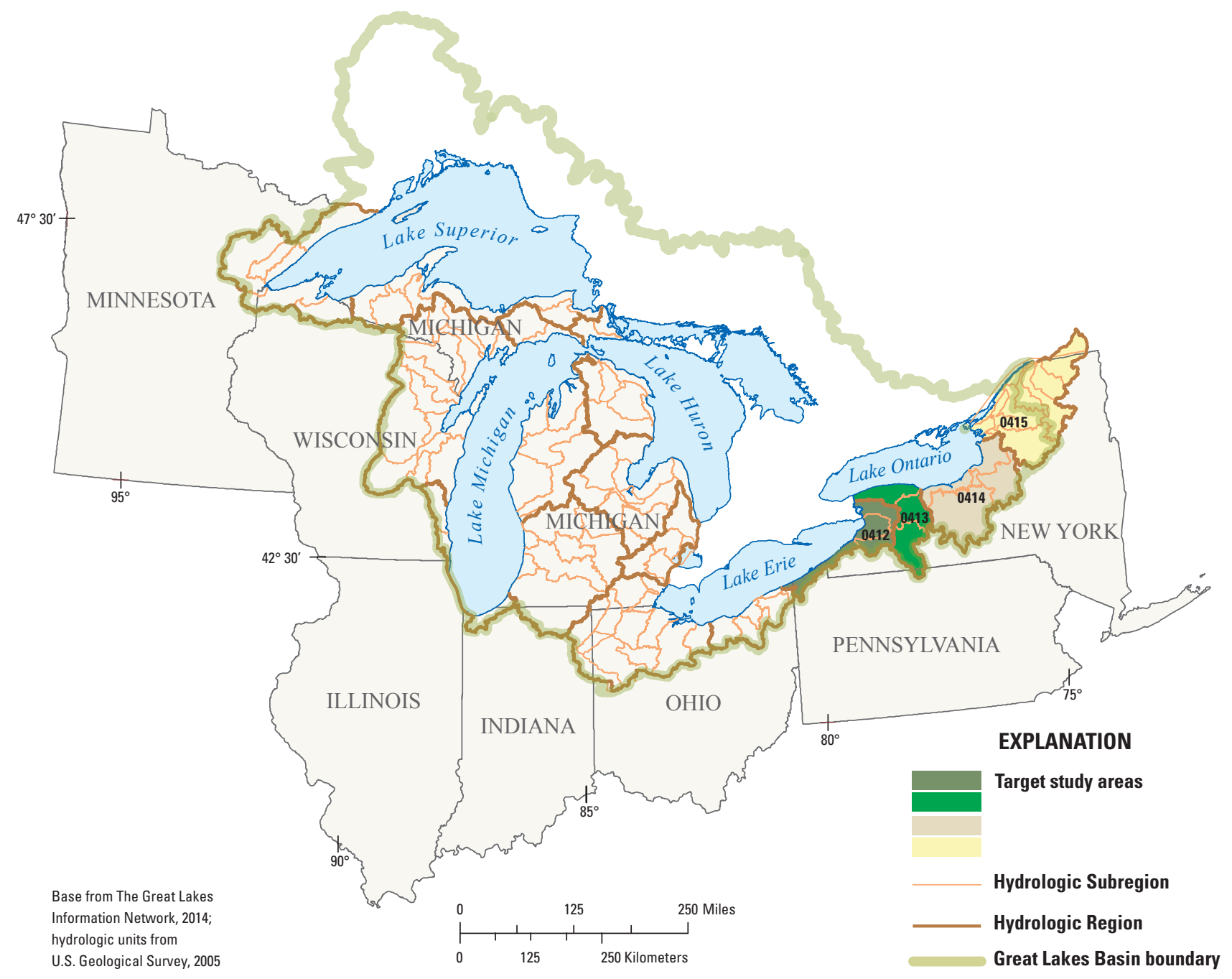

Figure 16. Location of study area 7 (hydrologic subregions 0412, 0413, 0414, and 0415) in the U.S. Great Lakes Basin. 
Table 15. Streamgaging stations considered for analysis of study area 7 (hydrologic subregions $0412,0413,0414$, and 0415 ) in the U.S. Great Lakes Basin, by water year.

$[\bullet$, station active and included in the analysis; ○, station active but not included in the analysis]

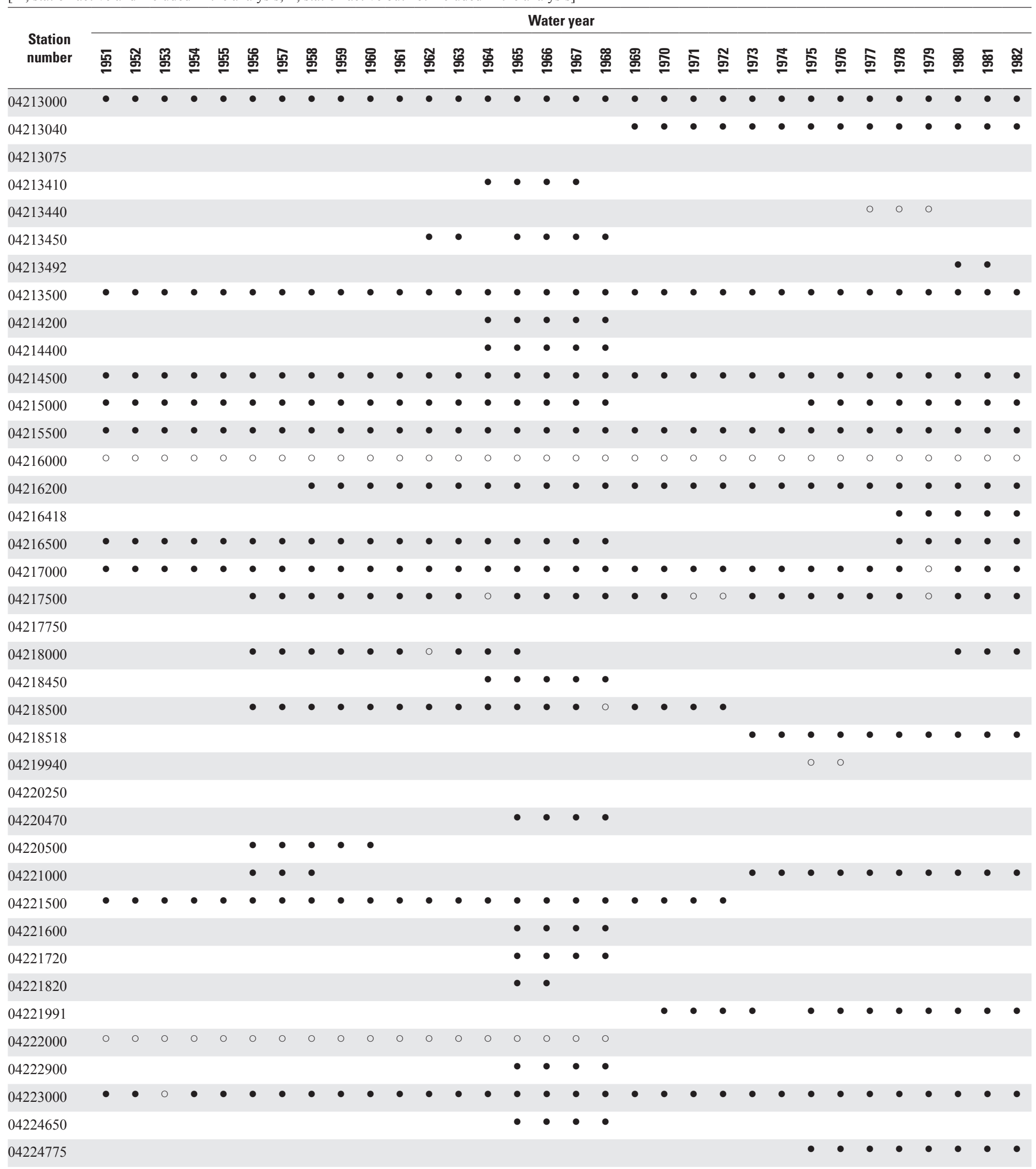


Table 15. Streamgaging stations considered for analysis of study area 7 (hydrologic subregions $0412,0413,0414$, and 0415 ) in the U.S. Great Lakes Basin, by water year.-Continued

$[\bullet$, station active and included in the analysis; $\odot$, station active but not included in the analysis]

Water year

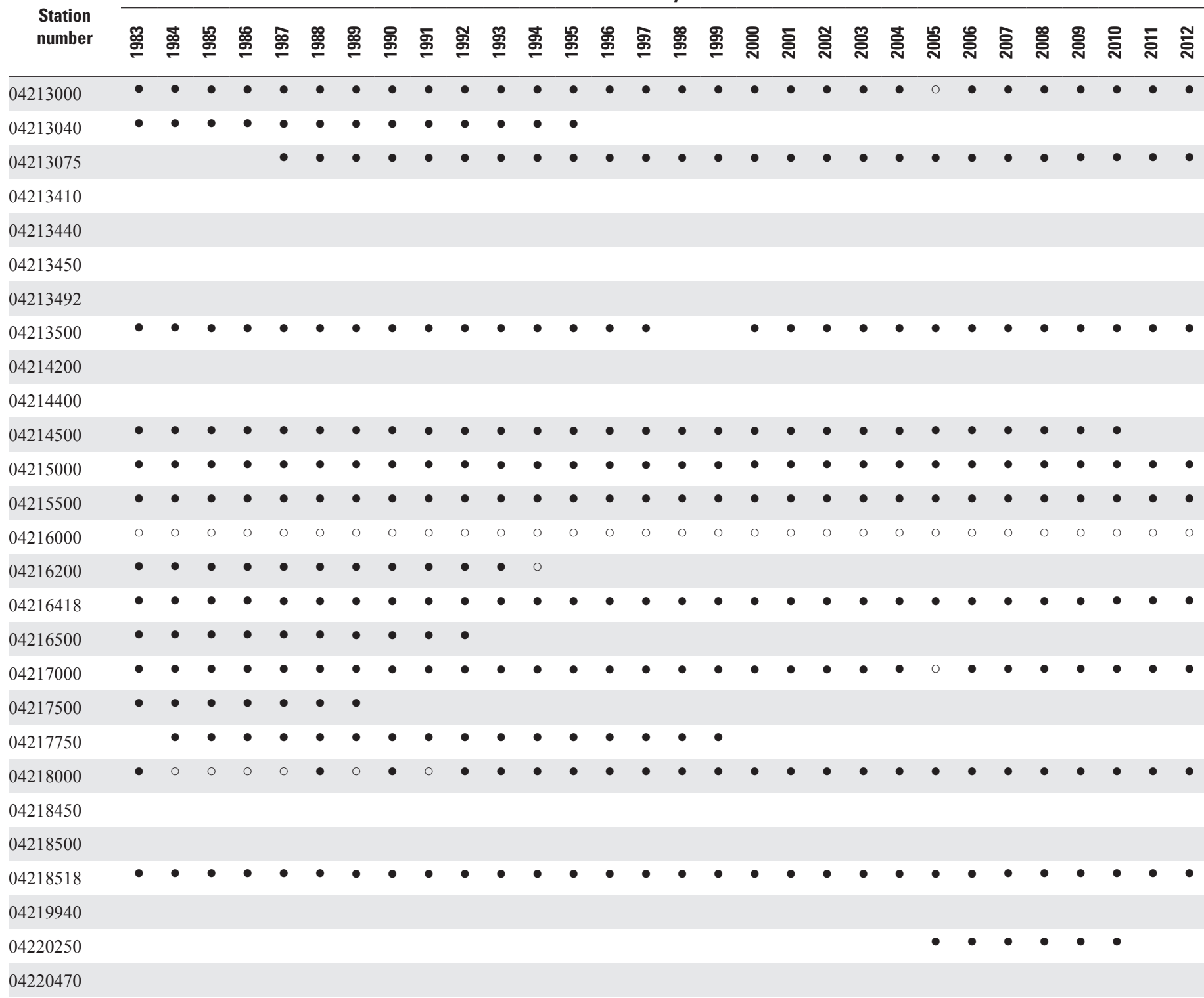

04220500

04221000

04221500

04221600

04221720

04221820

04221991

04222000

04222900

04223000

04224650

04224775 
Table 15. Streamgaging stations considered for analysis of study area 7 (hydrologic subregions $0412,0413,0414$, and 0415 ) in the U.S. Great Lakes Basin, by water year.-Continued

[ $\bullet$, station active and included in the analysis; ०, station active but not included in the analysis]

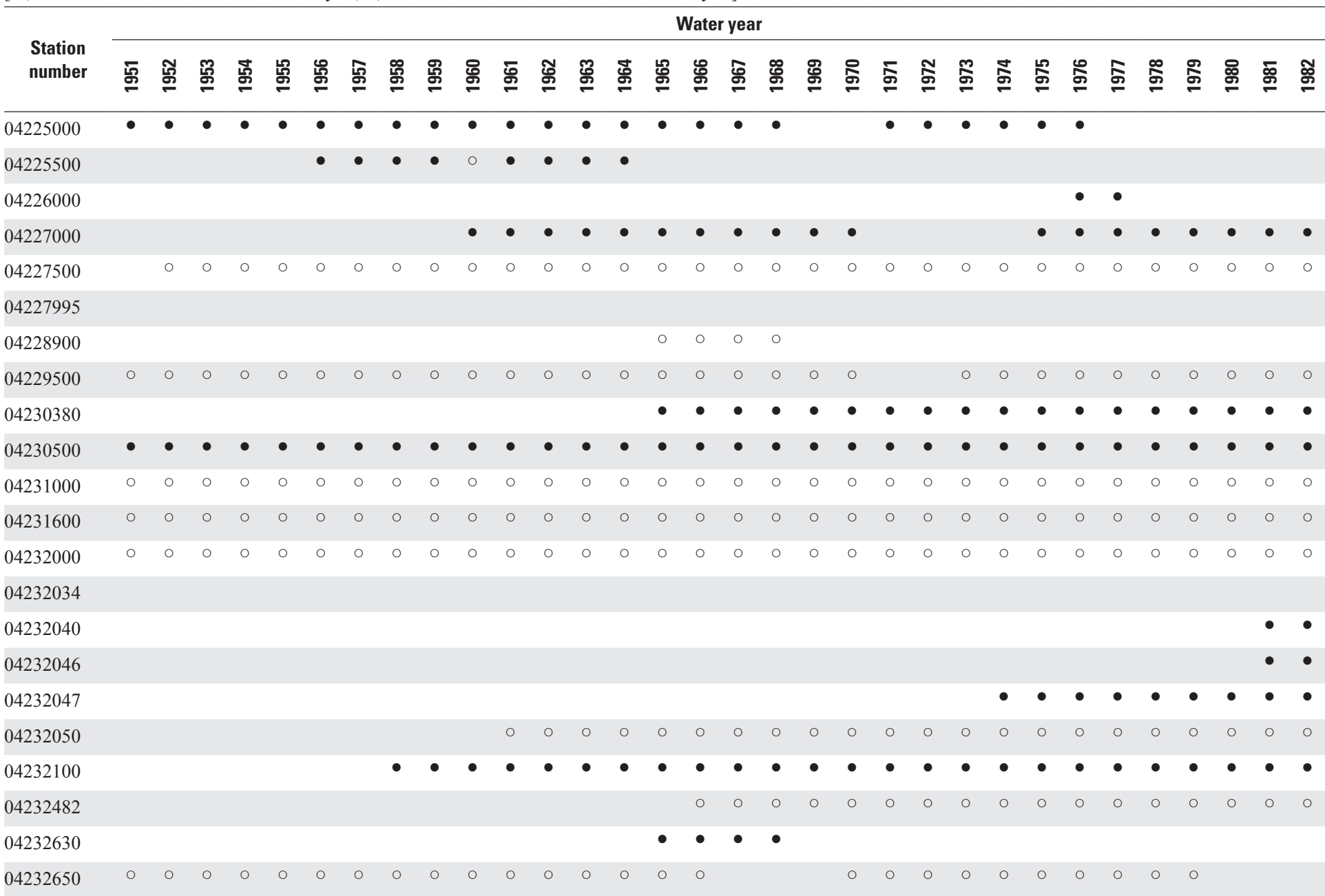

04232730

04233000

04233286

04233300

04233678

04233700

04234000

04234018

04234055

04234061

04234200

04234232

04234270

04235000

04235150

04235250

04235271 
Table 15. Streamgaging stations considered for analysis of study area 7 (hydrologic subregions $0412,0413,0414$, and 0415 ) in the U.S. Great Lakes Basin, by water year.-Continued

$[\bullet$, station active and included in the analysis; $\circ$, station active but not included in the analysis]

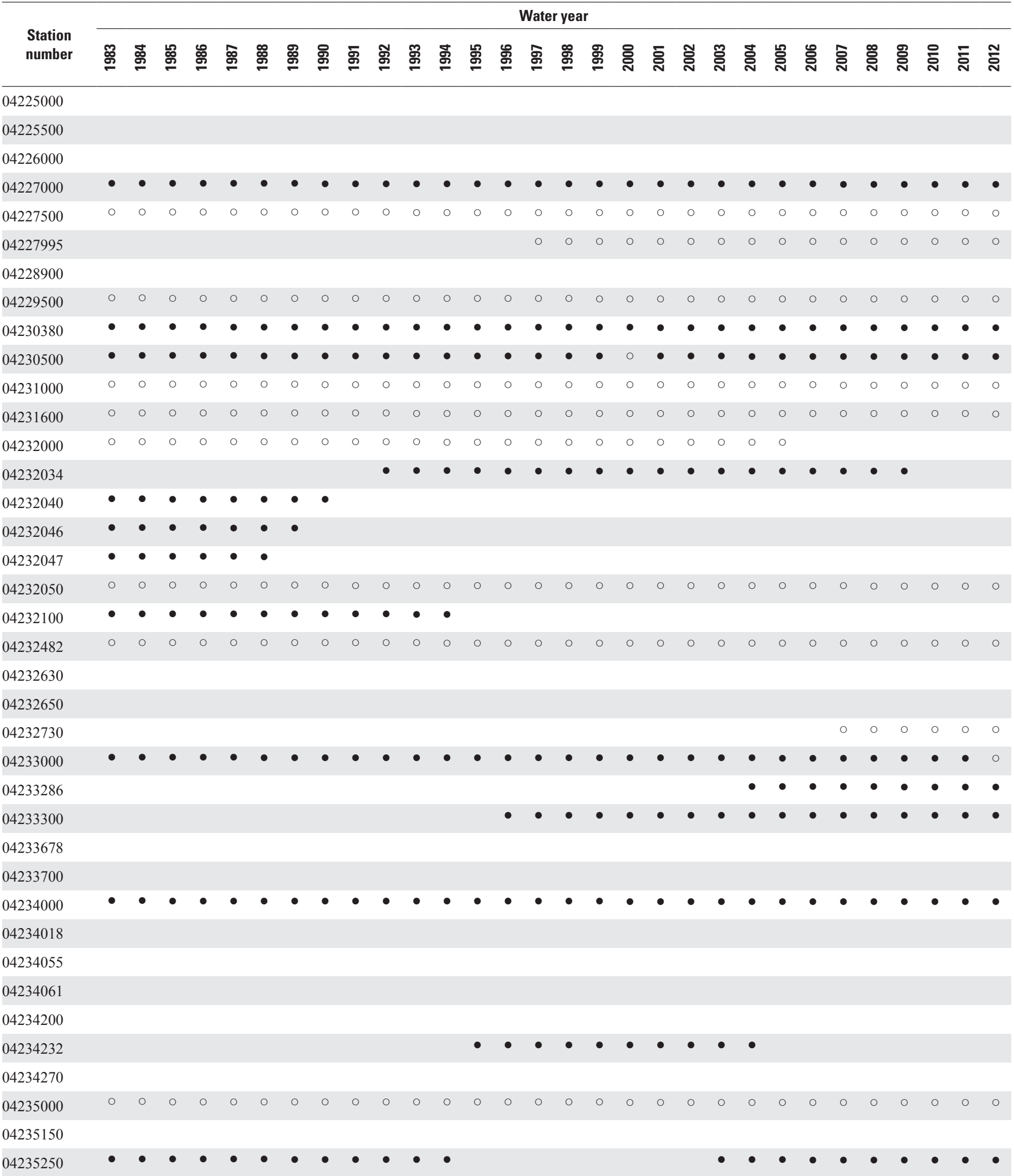

04235271 
Table 15. Streamgaging stations considered for analysis of study area 7 (hydrologic subregions $0412,0413,0414$, and 0415 ) in the U.S. Great Lakes Basin, by water year.-Continued

[ $\bullet$, station active and included in the analysis; ○, station active but not included in the analysis]

\section{Water year}

\begin{tabular}{|c|c|c|c|c|c|c|c|c|c|c|c|c|c|c|c|c|c|c|c|c|c|c|c|c|c|c|c|c|c|c|c|c|}
\hline $\begin{array}{l}\text { Station } \\
\text { number }\end{array}$ & 동 & ్ํํ & ஜூ్๊ & 范 & 농 & 냄 & 동 & 㔛 & 옴 & ळ్ & Б্ & ช్రా & ஜூ & 芯 & 농 & ஜ & ङ્ळ & ஜ̊ & 요 & 욤 & $\underset{\sigma}{\sigma}$ & $\underset{N}{\mathbb{N}}$ & হே & $\underset{\nabla}{\frac{\pi}{\sigma}}$ & $\frac{1}{\sigma}$ & $\stackrel{0}{\frac{0}{\sigma}}$ & E & $\stackrel{\infty}{\sigma}$ & $\frac{\Phi}{\sigma}$ & 吕 & চ্口- & ஐ̊ \\
\hline \multicolumn{33}{|l|}{04235276} \\
\hline 04235300 & & & & & & & & & & & O & $\bullet$ & $\bullet$ & $\bullet$ & $\bullet$ & $\bullet$ & $\bullet$ & $\bullet$ & & & & & & & & & & & & & & \\
\hline \multicolumn{33}{|l|}{04235440} \\
\hline 04235500 & $\circ$ & o & O & $\circ$ & 0 & o & o & O & o & $\circ$ & o & O & O & o & O & o & O & o & o & O & O & O & O & O & ० & ○ & O & $\circ$ & O & O & $\circ$ & o \\
\hline \multicolumn{33}{|l|}{04235600} \\
\hline \multicolumn{33}{|l|}{04235820} \\
\hline 04237500 & 0 & o & O & 0 & 0 & 0 & o & ० & 0 & 0 & o & O & 0 & o & 0 & o & O & 0 & 0 & 0 & 0 & O & 0 & 0 & O & 0 & 0 & 0 & 0 & 0 & 0 & o \\
\hline 04237944 & & & & & & & & & & & & & & & & & & & & & & & & & & & & & & & & \\
\hline 4237946 & & & & & & & & & & & & & & & & & & & & & & & & & & & & & & & & \\
\hline
\end{tabular}

04237962

04239000

04240010

04240100

04240105

04240120

04240145

04240150

04240180

04240200

04240300

04241200

04242500

04243500

04244000

04245000

04245200

04245236

04245250

04245840

04246500

04246601

04247000

04249000

04249910

04250200

04250750

04252000

04252500

04253000 
Table 15. Streamgaging stations considered for analysis of study area 7 (hydrologic subregions $0412,0413,0414$, and 0415 ) in the U.S. Great Lakes Basin, by water year.-Continued

$[\bullet$, station active and included in the analysis; $\circ$, station active but not included in the analysis]

\begin{tabular}{|c|c|c|c|c|c|c|c|c|c|c|c|c|c|c|c|c|c|c|c|c|c|c|c|c|c|c|c|c|c|c|}
\hline \multirow{2}{*}{$\begin{array}{l}\text { Station } \\
\text { number }\end{array}$} & \multicolumn{30}{|c|}{ Water year } \\
\hline & ஜ্口 & 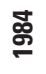 & 뇨 & ஜ & ஜ্口 & $\stackrel{\infty}{\infty}$ & ஜ유 & 윰 & চু & శ్ & ஜ్ㅁ & 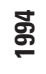 & 뇽 & ஜூ & శ্ন্ণ & 号 & 욤 & 유 & ธ్రి & ণั & ్ㅠ & 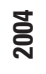 & 농 & ஸั & ్ㅗㅇ & 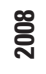 & 잉 & 음 & 호 & $\frac{\mathfrak{N}}{\text { ก }}$ \\
\hline 04235276 & & & & & $\bullet$ & $\bullet$ & $\bullet$ & $\bullet$ & $\bullet$ & $\bullet$ & $\bullet$ & - & $\bullet$ & & & & & & & & & & & & & & & & & \\
\hline \multicolumn{31}{|l|}{04235300} \\
\hline 04235440 & & & & & & & & & & & & & & & & & o & o & o & 0 & 0 & O & 0 & 0 & 0 & 0 & $\circ$ & 0 & 0 & o \\
\hline 04235500 & ○ & $\circ$ & $\circ$ & O & $\circ$ & O & O & O & o & o & o & o & $\circ$ & o & O & $\circ$ & & & & & & & & & & & & & & \\
\hline 04235600 & & & & & & & & & & & & & & & $\bullet$ & $\bullet$ & $\bullet$ & $\bullet$ & $\bullet$ & $\bullet$ & $\bullet$ & $\bullet$ & $\bullet$ & $\bullet$ & $\bullet$ & $\bullet$ & $\bullet$ & $\bullet$ & $\bullet$ & $\bullet$ \\
\hline 04235820 & & & & & & & & & & & & & & & $\circ$ & O & 0 & & & & & & & & & & & & & \\
\hline 04237500 & o & 0 & & O & & & & & & o & 0 & 0 & 0 & & & o & & & & & & & & & & & & & & \\
\hline 04237944 & & & & & & & & & & o & o & O & & & & & & & & & & & & & & & & & & \\
\hline 04237946 & & & & & & & & & & & 0 & 0 & 0 & o & 0 & 0 & o & 0 & o & 0 & 0 & ० & o & 0 & 0 & o & 0 & o & o & \\
\hline 04237962 & & & & & & & & & & & & & & & & & & & & $\bullet$ & $\bullet$ & $\bullet$ & $\bullet$ & $\bullet$ & $\bullet$ & $\bullet$ & $\bullet$ & $\bullet$ & $\bullet$ & $\bullet$ \\
\hline 04239000 & $\bullet$ & $\bullet$ & $\bullet$ & $\bullet$ & $\bullet$ & $\bullet$ & $\bullet$ & $\bullet$ & $\bullet$ & $\bullet$ & $\bullet$ & $\bullet$ & $\bullet$ & $\bullet$ & $\bullet$ & $\bullet$ & $\bullet$ & $\bullet$ & $\bullet$ & $\bullet$ & $\bullet$ & $\bullet$ & $\bullet$ & $\bullet$ & $\bullet$ & $\bullet$ & $\bullet$ & $\bullet$ & $\bullet$ & $\bullet$ \\
\hline 04240010 & ० & 0 & $\circ$ & $\circ$ & $\circ$ & o & ० & o & 0 & o & 0 & ○ & $\circ$ & O & O & ० & o & o & o & 0 & $\circ$ & $\circ$ & ० & $\circ$ & ० & o & o & o & o & o \\
\hline 04240100 & 0 & O & $\circ$ & 0 & $\circ$ & 0 & 0 & 0 & 0 & 0 & 0 & 0 & 0 & O & O & 0 & 0 & 0 & o & 0 & $\circ$ & O & ○ & & O & o & 0 & 0 & 0 & ० \\
\hline 04240105 & ० & O & o & $\circ$ & o & ० & o & O & ० & o & o & $\circ$ & ० & $\circ$ & 0 & $\circ$ & $\circ$ & $\circ$ & $\circ$ & 0 & $\circ$ & $\circ$ & $\circ$ & ० & $\circ$ & ० & o & o & ○ & o \\
\hline 04240120 & $\bullet$ & $\bullet$ & $\bullet$ & $\bullet$ & $\bullet$ & - & $\bullet$ & $\bullet$ & $\bullet$ & $\bullet$ & - & - & $\bullet$ & $\bullet$ & $\bullet$ & $\bullet$ & $\bullet$ & $\bullet$ & $\bullet$ & - & $\bullet$ & $\bullet$ & $\bullet$ & $\bullet$ & $\bullet$ & $\bullet$ & $\bullet$ & $\bullet$ & & \\
\hline 04240145 & ○ & & & & & & & & & & & & & & & & & & & & & & & & & & & & & \\
\hline 04240150 & • & & & & & & & & & & & & & & & & & & & & & & & & & & & & & \\
\hline 04240180 & o & o & o & o & o & o & o & o & ० & o & o & $\circ$ & $\circ$ & $\circ$ & 0 & $\circ$ & $\circ$ & $\circ$ & $\circ$ & 0 & $\circ$ & $\circ$ & $\circ$ & $\circ$ & $\circ$ & ० & ० & ० & ० & ० \\
\hline 04240200 & & & & & & & 0 & 0 & 0 & 0 & 0 & 0 & 0 & ० & 0 & 0 & & & & & & & & & 0 & & & & & \\
\hline 04240300 & 0 & 0 & 0 & 0 & 0 & 0 & 0 & 0 & 0 & 0 & 0 & 0 & 0 & 0 & 0 & 0 & 0 & 0 & 0 & 0 & 0 & 0 & 0 & 0 & 0 & 0 & 0 & 0 & 0 & O \\
\hline \multicolumn{31}{|l|}{04241200} \\
\hline 04242500 & 0 & 0 & & 0 & 0 & 0 & 0 & 0 & 0 & 0 & 0 & 0 & & & & & & & & & & & & & & & 0 & 0 & 0 & 0 \\
\hline 04243500 & $\bullet$ & $\bullet$ & $\bullet$ & $\bullet$ & $\bullet$ & $\bullet$ & $\bullet$ & $\bullet$ & $\bullet$ & $\bullet$ & $\bullet$ & $\bullet$ & $\bullet$ & $\bullet$ & $\bullet$ & $\bullet$ & $\bullet$ & $\bullet$ & $\bullet$ & $\bullet$ & $\bullet$ & $\bullet$ & $\bullet$ & $\bullet$ & $\bullet$ & $\bullet$ & $\bullet$ & $\bullet$ & $\bullet$ & $\bullet$ \\
\hline \multicolumn{31}{|l|}{04244000} \\
\hline 04245000 & $\bullet$ & $\bullet$ & $\bullet$ & $\bullet$ & & & & & & & & & & & & & & & & & & & & & & & & & & \\
\hline 04245200 & $\bullet$ & $\bullet$ & $\bullet$ & $\bullet$ & $\bullet$ & $\bullet$ & $\bullet$ & $\bullet$ & $\bullet$ & $\bullet$ & $\bullet$ & $\bullet$ & $\bullet$ & $\bullet$ & $\bullet$ & $\bullet$ & $\bullet$ & & & & & & & & & & & & & \\
\hline 04245236 & 0 & 0 & 0 & O & 0 & 0 & 0 & 0 & 0 & 0 & 0 & 0 & 0 & 0 & 0 & 0 & 0 & 0 & 0 & 0 & 0 & $\circ$ & 0 & 0 & $\circ$ & & & & & \\
\hline \multicolumn{31}{|l|}{04245250} \\
\hline \multicolumn{31}{|l|}{04245840} \\
\hline 04246500 & $\bullet$ & $\bullet$ & $\bullet$ & $\bullet$ & $\bullet$ & • & $\bullet$ & $\bullet$ & $\bullet$ & $\bullet$ & • & • & $\bullet$ & $\bullet$ & $\bullet$ & • & & & & & & & & & & & & & & \\
\hline 04246601 & & 0 & & & & & & & & & & & & & & & & & & & & & & & & & & & & \\
\hline 04247000 & & & & & & & & & & & & & & & & $\bullet$ & $\bullet$ & $\bullet$ & $\bullet$ & $\bullet$ & - & $\bullet$ & • & $\bullet$ & $\bullet$ & $\bullet$ & $\bullet$ & $\bullet$ & $\circ$ & ○ \\
\hline 04249000 & 0 & 0 & 0 & 0 & 0 & 0 & 0 & 0 & 0 & 0 & 0 & 0 & 0 & 0 & 0 & 0 & 0 & 0 & 0 & 0 & 0 & 0 & 0 & 0 & 0 & 0 & 0 & 0 & 0 & ० \\
\hline \multicolumn{31}{|l|}{04249910} \\
\hline 04250200 & & & & & & & & & & & & 0 & 0 & 0 & $\circ$ & 0 & 0 & 0 & 0 & 0 & 0 & 0 & 0 & 0 & 0 & 0 & 0 & 0 & 0 & ० \\
\hline 04250750 & o & $\circ$ & $\circ$ & O & $\circ$ & 0 & 0 & 0 & 0 & 0 & $\circ$ & 0 & & & & & & & & & 0 & 0 & $\circ$ & 0 & 0 & 0 & 0 & 0 & $\circ$ & ○ \\
\hline \multicolumn{31}{|l|}{04252000} \\
\hline 04252500 & $\bullet$ & $\bullet$ & $\bullet$ & $\bullet$ & $\bullet$ & $\bullet$ & $\bullet$ & $\bullet$ & $\bullet$ & $\bullet$ & $\bullet$ & $\bullet$ & $\bullet$ & $\bullet$ & $\bullet$ & $\bullet$ & $\bullet$ & $\bullet$ & $\bullet$ & $\bullet$ & $\bullet$ & $\bullet$ & $\bullet$ & $\bullet$ & $\bullet$ & $\bullet$ & $\bullet$ & $\bullet$ & $\bullet$ & $\bullet$ \\
\hline
\end{tabular}


Table 15. Streamgaging stations considered for analysis of study area 7 (hydrologic subregions $0412,0413,0414$, and 0415 ) in the U.S. Great Lakes Basin, by water year.-Continued

$[\bullet$, station active and included in the analysis; ○, station active but not included in the analysis]

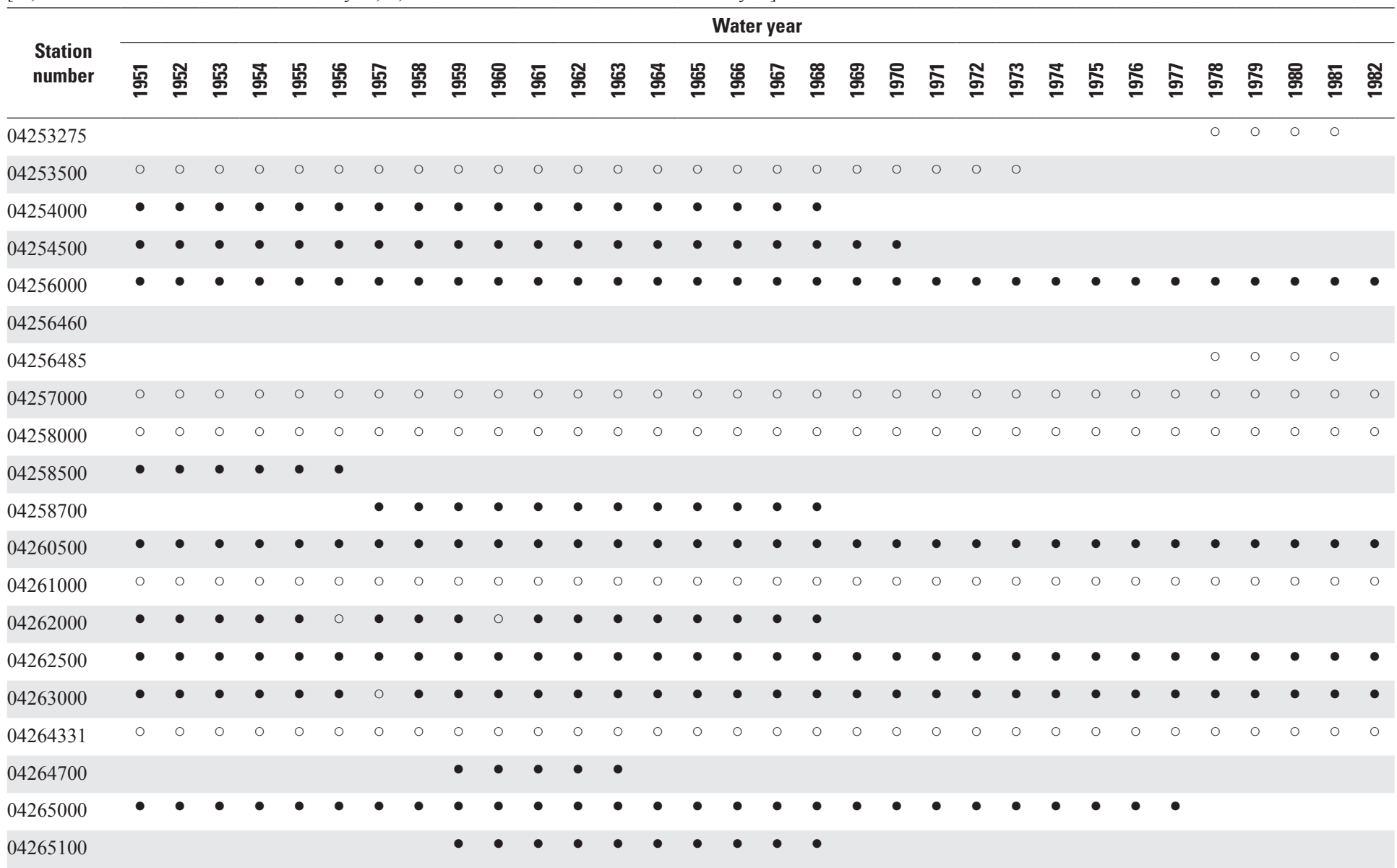

04265432

04265605

04266500

04267500

04268000

04268390

04268600

04268700

04268800

04269000

04269043

04269500

04270000

04270200

04270500

04270510

0422026250

0423204140

0423204141 
Table 15. Streamgaging stations considered for analysis of study area 7 (hydrologic subregions $0412,0413,0414$, and 0415 ) in the U.S. Great Lakes Basin, by water year.-Continued

$[\bullet$, station active and included in the analysis; $\odot$, station active but not included in the analysis]

Water year

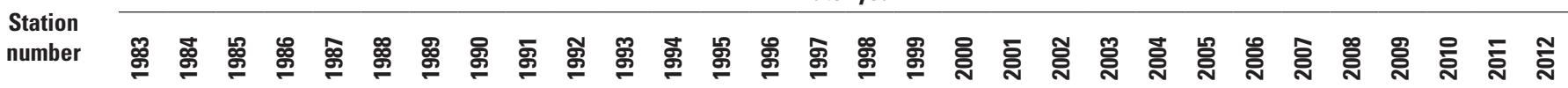

04253275

04253500

04254000

04254500

04256000

04256460

04256485

04257000

04258000

04258500

04258700

04260500

04261000

04262000

04262500

04263000

04264331

04264700

04265000

04265100

04265432

04265605

04266500

04267500

04268000

04268390

04268600

04268700

04268800

04269000

04269043

04269500

04270000

04270200

04270500

04270510

0422026250

0423204140

042320414 
Table 15. Streamgaging stations considered for analysis of study area 7 (hydrologic subregions $0412,0413,0414$, and 0415 ) in the U.S. Great Lakes Basin, by water year.-Continued

[ $\bullet$, station active and included in the analysis; $\circ$, station active but not included in the analysis]

\begin{tabular}{|c|c|c|c|c|c|c|c|c|c|c|c|c|c|c|c|c|c|c|c|c|c|c|c|c|c|c|c|c|c|c|c|c|}
\hline \multirow{2}{*}{$\begin{array}{l}\text { Station } \\
\text { number }\end{array}$} & \multicolumn{32}{|c|}{ Water year } \\
\hline & 돔 & ్ㅗㅁ & గొ్ & 壱 & 농 & 뜜ำ & 동 & 曲 & ్ㅗㅇ & 윰 & Б্ㅁ & ฐุ & ஜூ & すே & 용 & ஜே & क्ष & ஜ̊ & 용 & $\frac{R}{\sigma}$ & $\underset{\sigma}{\sigma}$ & $\underset{\mathbb{N}}{\stackrel{\Xi}{-}}$ & $\stackrel{\Re}{\mathscr{\sigma}}$ & $\stackrel{\leftarrow}{\frac{\pi}{\sigma}}$ & $\frac{\text { ñ }}{2}$ & $\stackrel{0}{\frac{\sigma}{\sigma}}$ & స్ & $\stackrel{\infty}{\sigma}$ & $\frac{\sigma}{\sigma}$ & 용 & бо & ๙ \\
\hline \multicolumn{33}{|l|}{0423204920} \\
\hline 0423205010 & & & & & & & & & & & & & & & & & & & & & & & & & & & & & & & & o \\
\hline 0423205023 & & & & & & & & & & & & & & & & & & & & & & & & & & & & & & & & o \\
\hline \multicolumn{33}{|l|}{0423205025} \\
\hline \multicolumn{33}{|l|}{0423368620} \\
\hline \multicolumn{33}{|l|}{0424014980} \\
\hline \multicolumn{33}{|l|}{0424016205} \\
\hline \multicolumn{33}{|l|}{0424016825} \\
\hline \multicolumn{33}{|l|}{0424016975} \\
\hline 0426545290 & & & & & & & & & & & & & & & & & & & & & & & & & & & & & $\bullet$ & $\bullet$ & & \\
\hline 0426545295 & & & & & & & & & & & & & & & & & & & & & & & & & & & & $\circ$ & 0 & 0 & $\circ$ & \\
\hline
\end{tabular}


Table 15. Streamgaging stations considered for analysis of study area 7 (hydrologic subregions $0412,0413,0414$, and 0415 ) in the U.S. Great Lakes Basin, by water year.-Continued

$[\bullet$, station active and included in the analysis; $\circ$, station active but not included in the analysis]

\begin{tabular}{|c|c|c|c|c|c|c|c|c|c|c|c|c|c|c|c|c|c|c|c|c|c|c|c|c|c|c|c|c|c|c|}
\hline \multirow{2}{*}{$\begin{array}{l}\text { Station } \\
\text { number }\end{array}$} & \multicolumn{30}{|c|}{ Water year } \\
\hline & ஜூ: & 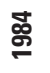 & 요 & ஜே & ळ্ & 吕 & 吕 & 욤 & Б্ & శ్ & ஜூ̆ & 冚 & 동 & ஜே & જூ & 哭 & 용 & ్ㅠ & ర్ర & ণ్రి & ఇ̊̊ & 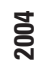 & 농 & ণ্రి & ్ㅗㅇ & 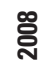 & 용 & 음 & Е్ & ำ \\
\hline 0423204920 & & & & & & & & & $\bullet$ & $\bullet$ & $\bullet$ & $\bullet$ & $\bullet$ & $\bullet$ & $\bullet$ & $\bullet$ & $\bullet$ & $\bullet$ & $\bullet$ & $\bullet$ & & & & & & & & & & \\
\hline 0423205010 & O & $\circ$ & $\circ$ & o & $\circ$ & $\circ$ & $\circ$ & $\circ$ & $\circ$ & $\circ$ & $\circ$ & ० & $\circ$ & $\circ$ & $\circ$ & $\circ$ & $\circ$ & ० & ० & ○ & $\circ$ & O & $\circ$ & $\circ$ & $\circ$ & $\circ$ & & & & \\
\hline 0423205023 & o & & & & & & & & & & & & & & & & & & & & & & & & & & & & & \\
\hline 0423205025 & & & & & & & & & $\circ$ & $\circ$ & $\circ$ & ○ & $\circ$ & $\circ$ & ○ & $\circ$ & $\circ$ & $\circ$ & $\circ$ & ० & & & & & & & & & & \\
\hline 0423368620 & & & & & & & & & & & & & & & & & & & & & & ० & & & & & & & & \\
\hline 0424014980 & & & & & & & & & & & & & & & & ० & ० & $\circ$ & $\circ$ & ० & ० & & & & & & & & & \\
\hline 0424016205 & $\bullet$ & & & & & & & & & & & & & & & & & & & & & & & & & & & & & \\
\hline 0424016825 & $\bullet$ & & & & & & & & & & & & & & & & & & & & & & & & & & & & & \\
\hline 0424016975 & $\bullet$ & & & & & & & & & & & & & & & & & & & & & & & & & & & & & \\
\hline \multicolumn{31}{|l|}{0426545290} \\
\hline 0426545295 & & & & & & & & & & & & & & & & & & & & & & & & & & & & & & \\
\hline
\end{tabular}


Table 16. Regression statistics used in the AFINCH (Analysis of Flows In Networks of CHannels) analysis for study area 7 (hydrologic subregions $0412,0413,0414$, and 0415) in the U.S. Great Lakes Basin, by month.

[-, indicates variable not included in monthly regression equation; GDD, growing degree days; RMSE, root mean square error; $\mathrm{R}^{2}$, coefficient of determination. See appendix tables for variable descriptions]

\begin{tabular}{|c|c|c|c|c|c|c|c|c|c|c|c|c|c|c|c|c|}
\hline \multirow[b]{2}{*}{ Month } & \multicolumn{13}{|c|}{ Regression coefficients } & \multicolumn{3}{|c|}{ Regression fit statistics } \\
\hline & Intercept & $\begin{array}{c}\text { Current } \\
\text { precipi- } \\
\text { tation }\end{array}$ & $\begin{array}{l}\text { Preceding } \\
\text { precipita- } \\
\text { tion }\end{array}$ & $\begin{array}{c}\text { Current } \\
\text { tempera- } \\
\text { ture }\end{array}$ & $\begin{array}{l}\text { Open } \\
\text { water }\end{array}$ & Forest & Developed & $\begin{array}{l}\text { Mean } \\
\text { slope }\end{array}$ & $\begin{array}{l}\text { Hydric group } \\
C, C D \text {, and D }\end{array}$ & $\begin{array}{c}\text { Coarse } \\
\text { outwash and } \\
\text { ice conract }\end{array}$ & $\begin{array}{l}\text { Coarse end } \\
\text { and ground } \\
\text { moraines }\end{array}$ & $\begin{array}{c}\text { Average } \\
\text { GDD }\end{array}$ & $\begin{array}{l}\text { Pasture } \\
\text { hay and } \\
\text { row crops }\end{array}$ & RMSE & F-statistic & $\mathbf{R}^{2}$ \\
\hline November & 0.462 & 0.179 & 0.107 & -0.011 & - & - & -0.221 & -0.726 & -0.030 & - & - & - & $5.1 \mathrm{E}-05$ & 0.266 & 709.8 & 0.658 \\
\hline January & 1.061 & 0.170 & 0.085 & 0.045 & - & - & -0.480 & -0.403 & -0.017 & 0.071 & - & - & - & 0.285 & 398.1 & 0.519 \\
\hline March & 2.051 & 0.155 & 0.046 & 0.039 & -3.962 & -0.520 & -1.088 & -0.817 & - & 0.253 & -0.203 & -0.263 & $-1.1 \mathrm{E}-04$ & 0.349 & 101.1 & 0.302 \\
\hline May & 1.208 & 0.165 & 0.067 & -0.024 & 1.809 & -0.383 & -0.543 & -0.778 & - & - & 0.178 & 0.140 & - & 0.222 & 847.6 & 0.748 \\
\hline July & 0.772 & 0.119 & 0.077 & -0.018 & 1.089 & -0.459 & - & -0.562 & - & -0.115 & 0.359 & 0.082 & - & 0.182 & 732.2 & 0.719 \\
\hline September & 0.061 & 0.152 & 0.094 & - & 1.058 & -0.305 & - & -0.671 & - & -0.133 & 0.304 & - & $4.0 \mathrm{E}-05$ & 0.224 & 761.5 & 0.703 \\
\hline
\end{tabular}

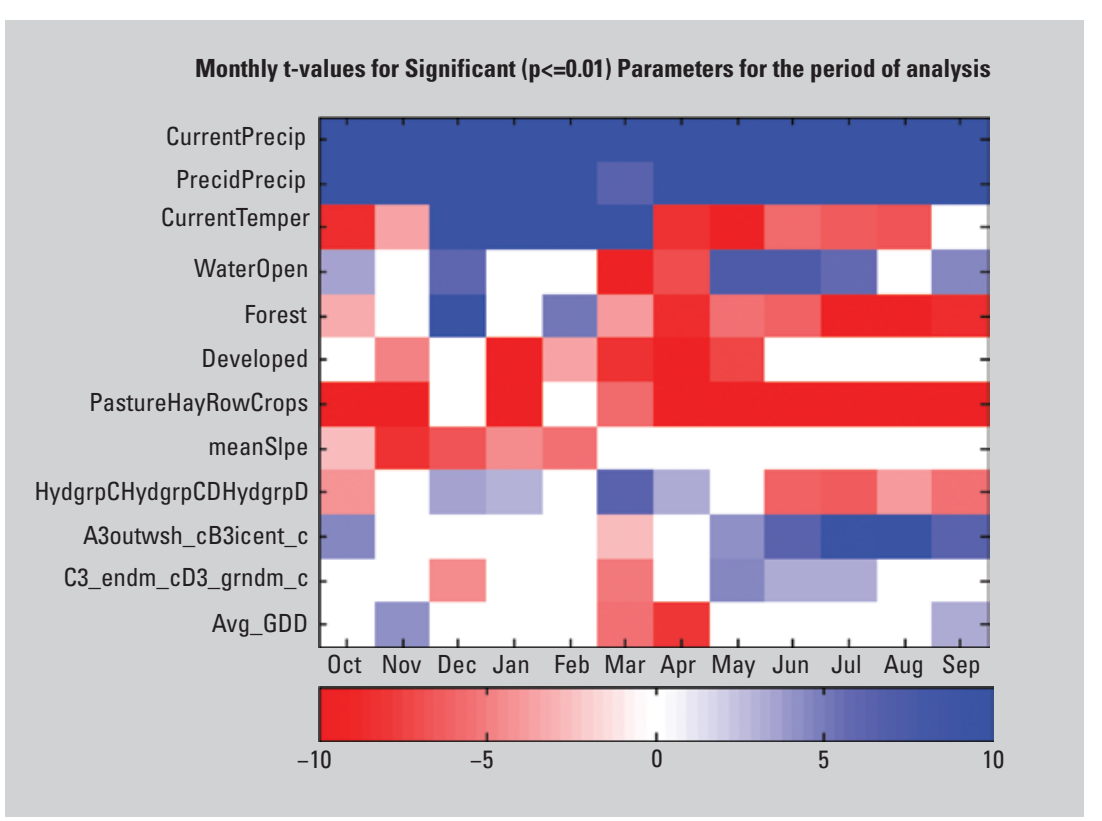

Figure 17. Image showing the t-value-indexed colors corresponding to each explanatory variable in the monthly regression equation for estimating water yield for study area 7 (hydrologic subregions 0412, 0413, 0414, and 0415) in the U.S. Great Lakes Basin. 


\section{Summary}

A regionally consistent estimate of streamflow provides unified information across the United States portion of the Great Lakes Basin for restoration, assessment, management, and conservation of stream ecosystems. Monthly water yields from catchments and corresponding flows for stream segments (flowlines) were estimated for water years 1951-2012 for the U.S. Great Lakes Basin by using the computer application Analysis of Flows In Networks of CHannels (AFINCH). The AFINCH approach to estimate ungaged flows is a two-step process. The predictor step is based on a user-specified multiple-linear-regression equation to estimate monthly yields using selected monthly climatic and basin characteristics data and flows at streamgages. The corrector step adjusts the regression estimates to match measured flows at the streamgages.

The 15 hydrologic subregions comprising the U.S. Great Lakes Basin were combined to form 7 study areas consisting of multiple subregions and (or) cataloguing units for analysis with AFINCH. Input data, explanatory variables, and regression details used in the estimation of water yields and flows are described for each study area. Data from 823 streamgages were compiled for the AFINCH analysis and were used to estimate monthly flows and water yields for 107,691 stream segments (flowlines) and 105,829 catchments in Hydrologic Region 04 for 1951-2012. These long-term flow series can be used to describe monthly flow-duration characteristics and to investigate trends for the stream segments in the U.S. Great Lakes Basin.

AFINCH utilizes the NHDPlus geospatial framework to provide a basis for integrating monthly flow measured at U.S. Geological Survey (USGS) streamgages, available wateruse data, monthly climatic data, land-cover characteristics, and catchment attributes described in user-defined shapefiles to develop sets of multiple-linear-regression equations for estimating monthly water yields. The explanatory variables describing monthly precipitation for the current month, monthly precipitation for the preceding month, and average monthly temperatures were included in the multiple-linearregression equations for all seven study areas. National landcover characteristics defining forest, pasture, and cultivated areas were significant explanatory variables in most of the study areas. NHDPlus variables defining stream order, as well as catchment elevation and (or) slope, and Soil Survey Geography (SSURGO) soil data also were significant explanatory variables in most of the study areas. Surficial areas of coarse outwash tended to be included in the regression equations for study areas in the southern and eastern parts of the U.S. Great Lakes Basin. Growing degree day data and areas with end and ground moraines also were important in several study areas. In general, coefficient of determination $\left(\mathrm{R}^{2}\right)$ values and F-statistics for the study areas were lowest in the late spring and early summer months (February-April) and higher in the late summer and early winter months (July-October). Considering a potential additional variable, such as an index relating to snow pack, would likely be significant and might improve the estimates during months of lower flow conditions. Regionally, however, these estimates present a reasonable, consistent, and defensible set of flow and yield estimates for the U.S. Great Lakes Basin.

\section{References Cited}

Council of Great Lakes Governors, 2005, Great LakesSt. Lawrence River Basin Water Resources Compact, accessed May 22, 2014, at http://www.cglg.org/projects/ water/docs/12-13-05/Great_Lakes-St_Lawrence_River_ Basin_Water_Resources_Compact.pdf.

Daly, Chris, and Taylor, G.H., 1998a, United States average monthly or annual precipitation, 1961-90: Corvallis, Ore., Spatial Climate Analysis Service at Oregon State University, accessed May 20, 2009, at http://www.climatesource. com/cd1/ppt_met_us.html.

Daly, Chris, and Taylor, G.H., 1998b, United States average monthly or annual mean temperature, 1961-90: Corvallis, Ore., Spatial Climate Analysis Service at Oregon State University, accessed May 20, 2009, at $h t t p: / / w w w$. climatesource.com/cd1/ppt_met_us.html.

Draper, N.R., and Smith, Harry, 1998, Applied regression analysis ( $3 \mathrm{~d}$ ed.): New York, John Wiley \& Sons, Inc., $736 \mathrm{p}$.

ESRI (Environmental Systems Research Institute), 2012, ArcMap 10.1: Redlands, Calif., available at $h t t p: / / w w w . e s r i$. $\mathrm{com} /$ software/arcgis/extensions/spatialanalyst/key-features/ statistical.

Fry, J.A., Xian, G., Jin, S., Dewitz, J.A., Homer, C.G., Yang, L., Barnes, C.A., Herold, N.D., and Wickham, J.D., 2011, Completion of the 2006 National Land Cover Database for the Conterminous United States: Photogrammetric Engineering and Remote Sensing, v. 77, no. 9, p. 858-864.

Gesch, Dean; Evans, Gayla; Mauck, James; Hutchinson, John; and Carswell, W.J., Jr., 2009, The National Map —Elevation: U.S. Geological Survey Fact Sheet 2009-3053, 4 p., (Also available at $h t t p: / / p u b s . u s g s . g o v / f s / 2009 / 3053 /$. 
Government of Canada and U.S. Environmental Protection Agency, 1995, The Great Lakes-An environmental atlas and resource book (3d ed.): U.S. Environmental Protection Agency EPA-905-B-95-00 1 and Environment Canada EN40-349/1995E, 46 p., map scales differ. (Also available at $h t t p: / / w w w . e p a . g o v / g l n p o / a t l a s /$.

Holtschlag, D.J., 2009, Application guide for AFINCH (Analysis of Flows In Networks of CHannels) described by NHDPlus: U.S. Geological Survey Scientific Investigations Report 2009-5188, 106 p. (Also available at http://pubs. usgs.gov/sir/2009/5188/.)

Horizon Systems Corporation, 2012, NHDPlus version 2.1, accessed June 2012, at http://www.horizon-systems.com/ NHDPlus/NHDPlusV2_home.php.

Horizon Systems Corporation, 2014, NHDPlusV2 flow table navigator toolbar, accessed May 27, 2014, at $h t t p: / /$ www.horizon-systems.com/NHDPlus/NHDPlusV2_tools. php\#NHDPlusV2.

Luukkonen, C.L., Blumer, S.P., Weaver, T.L., and Jean, Julie, 2004, Simulation of the ground-water-flow system in the Kalamazoo County area, Michigan: U.S. Geological Survey Scientific Investigations Report 2004-5054, 65 p.

McKay, Lucinda; Bondelid, Timothy; Dewald, Tommy; and others, 2012, NHDPlus version 2- User guide, 170 p., accessed August 28, 2014, at http://www.horizon-systems. com/NHDPlus/NHDPlusV2_documentation.php.

PRISM Climate Group, 2013, United States average mean annual precipitation and air temperature: Northwest Alliance for Computational Science and Engineering at Oregon State University, accessed August 1, 2013, at http://www. ocs.orst.edu/prism.

Seaber, P.R., Kapinos, F.P., and Knapp, G.L., 1987, Hydrologic unit maps (rev. 2007): U.S. Geological Survey WaterSupply Paper 2294, 63 p. (Also available at http://pubs. usgs.gov/wsp/wsp2294/.)

Strahler, A.N., 1957, Quantitative analysis of watershed geomorphology: Eos, Transactions American Geophysical Union, v. 38, no. 6, p. 913-920.

The MathWorks, Inc., 2010, MATLAB release 2010b: Natick, Mass., accessed August 28, 2014, at http://www.mathworks. com/products/new_products/release2010b.html.

The MathWorks, Inc., 2012, Matlab R2012a release highlights: Natick, Mass., accessed August 28, 2014, at http:// www.mathworks.com/products/new_products/release2012a. html.
U.S. Department of Agriculture-Natural Resources Conservation Service, 2009, National Watershed Boundary Dataset, accessed August 28, 2014, at http://www.nrcs.usda.gov/wps/ portal/nrcs/main/national/water/watersheds/dataset/.

U.S. Department of Agriculture-Natural Resources Conservation Service, 2011, Web Soil Survey, accessed November 8, 2011, at http://websoilsurvey.nrcs.usda.gov/.

U.S. Environmental Protection Agency, 2001, EPA/OW Permit Compliance System for BASINS Version 3 in CONUS, accessed August 28, 2014, at http://water.epa.gov/scitech/ datait/models/basins/pcs3.cfm.

U.S. Environmental Protection Agency, 2014, Great Lakes, accessed May 27, 2014, at http://www.epa.gov/greatlakes/.

U.S. Geological Survey, 2008, Hydrologic unit maps-What are hydrologic units?, accessed November 24, 2008, at http://water.usgs.gov/GIS/huc.html.

U.S. Geological Survey, 2009a, National Hydrography Dataset, available at $h t t p: / / n h d . u s g s . g o v /$.

U.S. Geological Survey, 2009b, National Elevation Dataset, available at $h t t p: / / n e d . u s g s . g o v /$.

U.S. Geological Survey, 2011, National Gap Analysis Program (GAP) - Land cover data and modeling, accessed May 2011, at http://gapanalysis.usgs.gov/gaplandcover/data.

Wahl, K.L., Thomas, W.O., Jr., and Hirsch, R.M., 1995, Stream-gaging program of the U.S. Geological Survey: U.S. Geological Survey Circular 1123, accessed May 27, 2014, at http://pubs.usgs.gov/circ/circ1123/index.html.

Waples, J.T.; Eadie, Brian; Klump, J.V.; Squires, Margaret; Cotner, James; and McKinley, Galen, 2008, Chapter 7The Laurentian Great Lakes, in Hales, Burke; Cai, Wei-Jun; Mitchell, B.G., Sabine, C.L., and Schofield, Oscar, eds., North American Continental Margins-A Synthesis and Planning Workshop-Report of the North American Continental Margins Working Group for the U.S. Carbon Cycle Scientific Steering Group and Interagency Working Group: Washington, D.C., U.S. Carbon Cycle Science Program, p. 73-81, accessed May 27, 2014, at http://www.glerl.noaa. gov/pubs/fulltext/2008/20080024.pdf. 


\section{U.S. Geological Survey Water-Data Reports for Michigan and the St. Lawrence River Basin}

\section{Regional compilations (listed chronologically)}

Wells, J.V.B., 1958, Compilation of records of surface waters of the United States through September 1950_Part 4, St. Lawrence River Basin: U.S. Geological Survey Water-Supply Paper 1307, 397 p.

Hendricks, E.L., 1964, Compilation of records of surface waters of the United States, October 1950 to September 1960 - Part 4, St. Lawrence River Basin: U.S. Geological Survey Water-Supply Paper 1727, 379 p.

U.S. Geological Survey, 1971, Surface water supply of the United States, 1961-65-Part 4, St. Lawrence River Basin-Volume 1, Basins of streams tributary to Lakes Superior, Michigan, and Huron: U.S. Geological Survey Water-Supply Paper 1911, 651 p.

U.S. Geological Survey, 1974, Surface water supply of the United States, 1966-70_Part 4, St. Lawrence River Basin-Volume 1, Basins of streams tributary to Lakes Superior, Michigan and Huron: U.S. Geological Survey Water-Supply Paper 2111, 754 p.

\section{Michigan data only (listed chronologically)}

U.S. Geological Survey, 1961-4, Surface water records of Michigan [annual data reports, water years 1961-4].

U.S. Geological Survey, 1965-75, Water resources data for Michigan, Part 1, Surface water records [annual data reports, water years 1965-74].

U.S. Geological Survey, 1976-82, Water resources data, Michigan, water years 1975-81: U.S. Geological Survey Water-Data Reports MI-75-1 through MI-81-1.

Miller, J.B., Oberg, J.L., and Sieger, Theodore, Jr., 1983-85, Water resources data, Michigan, water years 1982-84: U.S. Geological Survey Water-Data Reports MI-82-1 through MI-84-1.

Miller, J.B., Oberg, J.L., and Failing, J.C., 1986, Water resources data, Michigan, water year 1985: U.S. Geological Survey Water-Data Report MI-85-1, 297 p.
Miller, J.B., Failing, J.C., and Larson, W.W., 1987, Water resources data, Michigan, water year 1986: U.S. Geological Survey Water-Data Report MI-86-1, 353 p.

Blumer, S.P., Failing, J.C., Larson, W.W., Whited, C.R., and LeuVoy, R.L., 1988-90, Water resources data, Michigan, water years 1987-89: U.S. Geological Survey Water-Data Reports MI-87-1 through MI-89-1.

Blumer, S.P., Larson, W.W., Minnerick, R.J., Whited, C.R., and LeuVoy, R.L., 1991-92, Water resources data, Michigan, water years 1990-91: U.S. Geological Survey WaterData Reports MI-90-1 through MI-91-1.

Blumer, S.P., Behrendt, T.E., Larson, W.W., Minnerick, R.J., LeuVoy, R.L., and Whited, C.R., 1993-94, Water resources data, Michigan, water years 1992-93: U.S. Geological Survey Water-Data Reports MI-92-1 through MI-93-1.

Blumer, S.P., Behrendt, T.E., Ellis, J.M., Minnerick, R.J., LeuVoy, R.L., and Whited, C.R., 1995-2004, Water resources data, Michigan, water years 1994-2003: U.S. Geological Survey Water-Data Reports MI-94-1 through MI-03-1.

Blumer, S.P., Behrendt, Whited, C.R., T.E., Ellis, J.M., Minnerick, R.J., and LeuVoy, R.L., 2005, Water resources data, Michigan, water year 2004: U.S. Geological Survey WaterData Report MI-04-1, 496 p.

Blumer, S.P., Whited, C.R., Ellis, J.M., Minnerick, R.J., and LeuVoy, R.L., 2006, Water resources data, Michigan, water year 2005: U.S. Geological Survey Water-Data Report MI-05-1, $572 \mathrm{p}$.

U.S. Geological Survey, 2007-13, Water-resources data for the United States, water years 2006-12 [annual data compilations for stations 04034000 Bond Falls Reservoir near Paulding, Mich.; 04033500 Bond Falls Canal near Paulding, Mich.; 04057811 Greenwood Reservoir near Greenwood, Mich.; 04057814 Greenwood Release near Greenwood, Mich.; 04057813 Greenwood Diversion near Greenwood, Mich.; 04058000 Middle Branch Escanaba River near Ishpeming, Mich.; 04058190 Schweitzer Reservoir near Palmer, Mich.; and 04058400 Goose Lake Outlet near Sands Station, Mich.; accessed July 5, 2011, at http://wdr. water.usgs.gov/. 
Table 1-1. Revisions to NHDPlus flowline value-added attribute (VAA) information.

$[--$, not changed]

\begin{tabular}{|c|c|c|c|c|c|c|c|c|c|c|c|}
\hline \multirow{2}{*}{ Subregion } & \multirow{2}{*}{ ComID } & \multicolumn{5}{|c|}{ Original values } & \multicolumn{5}{|c|}{ Modified values } \\
\hline & & FromNode & ToNode & Hydroseq & Divergence & Startflag & FromNode & ToNode & Hydroseq & Divergence & Startflag \\
\hline \multirow[t]{4}{*}{0401} & 1798775 & -- & -- & 90072931 & 1 & -- & -- & -- & 90072920 & 0 & -- \\
\hline & 1798903 & 90003017 & 90003052 & 90072920 & 2 & 0 & 9003052 & 9003017 & 90072931 & 0 & 1 \\
\hline & 1771252 & -- & -- & -- & 1 & -- & -- & -- & -- & 0 & -- \\
\hline & 1772034 & -- & -- & -- & 2 & 0 & -- & -- & -- & 0 & 1 \\
\hline \multirow[t]{14}{*}{0402} & 11951467 & 90021466 & 90021629 & 90046042 & 1 & -- & 90021629 & 90021466 & 90046053 & 0 & -- \\
\hline & 11952189 & 90021629 & 90021470 & 90040541 & -- & -- & 90021470 & 90021629 & 90046185 & -- & -- \\
\hline & 11951469 & 90021470 & 90021630 & 90036575 & -- & -- & 90021630 & 90021470 & 90046186 & -- & -- \\
\hline & 11952191 & 90021630 & 90021472 & 90033483 & -- & -- & 90021472 & 90021630 & 90046187 & -- & -- \\
\hline & 11951473 & 90021472 & 90021631 & 90031008 & -- & -- & 90021631 & 90021472 & 90046188 & -- & -- \\
\hline & 11952193 & 90021631 & 90021628 & 90028979 & -- & 0 & 90021628 & 90021631 & 90046189 & -- & 1 \\
\hline & 11951459 & -- & -- & -- & 1 & -- & -- & -- & -- & 0 & -- \\
\hline & 11930450 & -- & -- & -- & 1 & 0 & -- & -- & -- & 0 & 1 \\
\hline & 11930458 & -- & -- & -- & 2 & -- & -- & -- & -- & 0 & -- \\
\hline & 11929798 & -- & -- & -- & 1 & -- & -- & -- & -- & 2 & -- \\
\hline & 11929802 & -- & -- & -- & 2 & -- & -- & -- & -- & 1 & -- \\
\hline & 11930458 & -- & -- & -- & 2 & -- & -- & -- & -- & 0 & -- \\
\hline & 11930450 & 90019836 & 90023688 & -- & 1 & -- & 90023688 & 90019836 & -- & 0 & -- \\
\hline & 12027444 & -- & -- & -- & -- & 0 & -- & -- & -- & -- & 1 \\
\hline \multirow[t]{14}{*}{0403} & 14444692 & -- & -- & -- & 1 & -- & -- & -- & -- & 0 & -- \\
\hline & 6820848 & -- & -- & -- & 1 & 0 & -- & -- & -- & 0 & 1 \\
\hline & 6849469 & -- & -- & -- & 2 & -- & -- & -- & -- & 0 & -- \\
\hline & 11959514 & -- & -- & -- & -- & 0 & -- & -- & -- & -- & 1 \\
\hline & 14444674 & -- & -- & -- & -- & -- & -- & -- & -- & -- & -- \\
\hline & 9027663 & -- & -- & -- & 2 & 0 & -- & -- & -- & 0 & 1 \\
\hline & 6809298 & -- & -- & -- & 1 & -- & -- & -- & -- & 0 & -- \\
\hline & 14444312 & -- & -- & -- & -- & 0 & -- & -- & -- & -- & 1 \\
\hline & 12006809 & -- & -- & -- & 2 & -- & -- & -- & -- & 1 & -- \\
\hline & 12006813 & -- & -- & -- & 1 & -- & -- & -- & -- & 2 & -- \\
\hline & 6865517 & -- & -- & -- & 1 & 0 & -- & -- & -- & 0 & 1 \\
\hline & 6866125 & -- & -- & -- & 2 & -- & -- & -- & -- & 0 & -- \\
\hline & 6821620 & -- & -- & -- & 2 & -- & -- & -- & -- & 1 & -- \\
\hline & 6821578 & -- & -- & -- & 1 & -- & -- & -- & -- & 2 & -- \\
\hline
\end{tabular}


Table 1-1. Revisions to NHDPlus flowline value-added attribute (VAA) information.-Continued

$[--$, not changed]

\begin{tabular}{|c|c|c|c|c|c|c|c|c|c|c|c|}
\hline \multirow{2}{*}{ Subregion } & \multirow{2}{*}{ ComID } & \multicolumn{5}{|c|}{ Original values } & \multicolumn{5}{|c|}{ Modified values } \\
\hline & & FromNode & ToNode & Hydroseq & Divergence & Startflag & FromNode & ToNode & Hydroseq & Divergence & Startflag \\
\hline \multirow{7}{*}{$\begin{array}{c}0403 \\
\text { (continued) }\end{array}$} & 12174040 & -- & -- & -- & 2 & 0 & -- & -- & -- & 0 & 1 \\
\hline & 12174028 & -- & -- & -- & 1 & -- & -- & -- & -- & 0 & -- \\
\hline & 9036437 & -- & -- & -- & 2 & 0 & -- & -- & -- & 0 & 1 \\
\hline & 9036449 & -- & -- & -- & 1 & -- & -- & -- & -- & 0 & -- \\
\hline & 11959396 & -- & -- & 90098082 & -- & 1 & -- & -- & 90017082 & -- & 0 \\
\hline & 11959398 & -- & -- & -- & -- & 0 & -- & -- & -- & -- & 1 \\
\hline & 11959926 & -- & 90021774 & -- & -- & -- & -- & 90082911 & -- & -- & -- \\
\hline \multirow[t]{2}{*}{0404} & 3397118 & -- & -- & -- & 2 & -- & -- & -- & -- & 0 & -- \\
\hline & 3396784 & -- & -- & -- & 1 & 0 & -- & -- & -- & 0 & 1 \\
\hline \multirow[t]{11}{*}{0405} & 12261958 & -- & -- & -- & 1 & -- & -- & -- & -- & 0 & -- \\
\hline & 12260476 & -- & -- & -- & 2 & 0 & -- & -- & -- & 0 & 1 \\
\hline & 13032275 & 90030086 & 90040059 & 90051985 & 2 & -- & 90040059 & 90030086 & 90044497 & 0 & -- \\
\hline & 13032045 & 90040059 & 90040060 & 90044497 & -- & 0 & 90040060 & 90040059 & 90051985 & -- & 1 \\
\hline & 12233796 & -- & -- & -- & 1 & -- & -- & -- & -- & 0 & -- \\
\hline & 12260238 & -- & -- & -- & 1 & -- & -- & -- & -- & 0 & -- \\
\hline & 12263234 & -- & -- & -- & 2 & 0 & -- & -- & -- & 0 & 1 \\
\hline & 12258396 & -- & -- & -- & 1 & 0 & -- & -- & -- & 0 & 1 \\
\hline & 12258498 & -- & -- & -- & 2 & -- & -- & -- & -- & 0 & -- \\
\hline & 12263190 & -- & -- & -- & 2 & 0 & -- & -- & -- & 0 & 1 \\
\hline & 12260112 & -- & -- & -- & 1 & -- & -- & -- & -- & 0 & -- \\
\hline \multirow[t]{4}{*}{0406} & 12222264 & 90029225 & 90029214 & 90067021 & 2 & 0 & 90029214 & 90029225 & 90067041 & 0 & 1 \\
\hline & 12221900 & -- & -- & -- & 1 & -- & -- & -- & -- & 0 & -- \\
\hline & 12222160 & -- & -- & -- & 2 & -- & -- & -- & -- & 0 & -- \\
\hline & 12221662 & -- & -- & -- & 1 & -- & -- & -- & -- & 0 & -- \\
\hline \multirow[t]{4}{*}{0407} & 12502899 & -- & -- & -- & 1 & -- & -- & -- & -- & 2 & -- \\
\hline & 12502901 & -- & -- & -- & 0 & -- & -- & -- & -- & 1 & -- \\
\hline & 12962413 & -- & -- & -- & 2 & 0 & -- & -- & -- & 0 & 1 \\
\hline & 12962487 & -- & -- & -- & 1 & -- & -- & -- & -- & 0 & -- \\
\hline
\end{tabular}


Table 1-1. Revisions to NHDPlus flowline value-added attribute (VAA) information.-Continued

$[--$, not changed]

\begin{tabular}{|c|c|c|c|c|c|c|c|c|c|c|c|}
\hline \multirow{2}{*}{ Subregion } & \multirow{2}{*}{ ComID } & \multicolumn{5}{|c|}{ Original values } & \multicolumn{5}{|c|}{ Modified values } \\
\hline & & FromNode & ToNode & Hydroseq & Divergence & Startflag & FromNode & ToNode & Hydroseq & Divergence & Startflag \\
\hline \multirow[t]{32}{*}{0408} & 12944858 & -- & -- & -- & 2 & -- & -- & -- & -- & 0 & -- \\
\hline & 12944468 & 90036462 & 90036538 & & 1 & 0 & 90036538 & 90036462 & & 0 & 1 \\
\hline & 12944860 & -- & -- & -- & 1 & 0 & -- & -- & -- & 0 & 1 \\
\hline & 13046031 & -- & -- & -- & 2 & 0 & -- & -- & -- & 0 & 1 \\
\hline & 13016527 & -- & -- & 90044601 & -- & -- & -- & -- & 90018387 & -- & -- \\
\hline & 13016547 & -- & -- & 90052133 & 1 & -- & -- & -- & 90018934 & 0 & -- \\
\hline & 13016565 & 90038914 & 90039104 & 90052132 & 2 & -- & 90039104 & 90038914 & 90019518 & 0 & -- \\
\hline & 13019475 & 90039104 & 90039109 & 90044580 & -- & -- & 90039109 & 90039104 & 90020159 & -- & -- \\
\hline & 13019493 & 90039109 & 90039117 & 90035767 & -- & -- & 90039117 & 90039109 & 90020830 & -- & -- \\
\hline & 13019523 & 90039117 & 90039292 & 90032845 & -- & -- & 90039292 & 90039117 & 90021565 & -- & -- \\
\hline & 13020129 & 90039292 & 90039294 & 90030478 & -- & -- & 90039294 & 90039292 & 90022385 & -- & -- \\
\hline & 13020133 & 90039294 & 90039129 & -- & -- & -- & 90039129 & 90039294 & -- & -- & -- \\
\hline & 13019559 & 90039129 & 90039298 & 90022385 & -- & -- & 90039298 & 90039129 & 90030478 & -- & -- \\
\hline & 13020141 & 90039298 & 90039135 & 90021565 & -- & -- & 90039135 & 90039298 & 90032845 & -- & -- \\
\hline & 13019577 & 90039135 & 90039138 & 90020830 & -- & -- & 90039138 & 90039135 & 90035500 & -- & -- \\
\hline & 13019583 & 90039138 & 90039142 & 90020159 & -- & -- & 90039142 & 90039138 & 90035550 & -- & -- \\
\hline & 13019591 & 90039142 & 90039300 & 90019518 & -- & -- & 90039300 & 90039142 & 90044601 & -- & -- \\
\hline & 13020145 & 90039300 & 90039299 & 90018934 & -- & 0 & 90039299 & 90039300 & 90052132 & -- & 1 \\
\hline & 13020143 & -- & -- & 90018387 & -- & 0 & -- & -- & 90052133 & -- & 1 \\
\hline & 13019637 & -- & -- & 90052102 & -- & -- & -- & -- & 90064410 & -- & -- \\
\hline & 13020157 & -- & -- & 90044561 & -- & -- & -- & -- & 90064405 & -- & -- \\
\hline & 13019631 & -- & -- & 90039494 & -- & -- & -- & -- & 90064400 & -- & -- \\
\hline & 13020155 & -- & -- & 90035756 & -- & -- & -- & -- & 90064395 & -- & -- \\
\hline & 13019605 & -- & -- & 90032841 & -- & -- & -- & -- & 90064390 & -- & -- \\
\hline & 13020147 & -- & -- & 90030477 & -- & -- & -- & -- & 90064385 & -- & -- \\
\hline & 13007650 & -- & -- & -- & 1 & 0 & -- & -- & -- & 0 & 1 \\
\hline & 13007796 & -- & -- & -- & 1 & 0 & -- & -- & -- & 0 & 1 \\
\hline & 13007622 & -- & -- & -- & 1 & 0 & -- & -- & -- & 0 & 1 \\
\hline & 13020539 & -- & -- & -- & 1 & 0 & -- & -- & -- & 0 & 1 \\
\hline & 13031999 & -- & -- & -- & 2 & -- & -- & -- & -- & 0 & -- \\
\hline & 13017263 & -- & -- & -- & 1 & -- & -- & -- & -- & 0 & -- \\
\hline & 13028773 & -- & -- & -- & 2 & 0 & -- & -- & -- & 0 & 1 \\
\hline
\end{tabular}


Table 1-1. Revisions to NHDPlus flowline value-added attribute (VAA) information.-Continued

$[--$, not changed $]$

\begin{tabular}{|c|c|c|c|c|c|c|c|c|c|c|c|}
\hline \multirow{2}{*}{ Subregion } & \multirow{2}{*}{ ComID } & \multicolumn{5}{|c|}{ Original values } & \multicolumn{5}{|c|}{ Modified values } \\
\hline & & FromNode & ToNode & Hydroseq & Divergence & Startflag & FromNode & ToNode & Hydroseq & Divergence & Startflag \\
\hline \multirow{5}{*}{$\begin{array}{c}0408 \\
\text { (continued) }\end{array}$} & 13029155 & -- & -- & -- & 2 & 0 & -- & -- & -- & 0 & 1 \\
\hline & 13028795 & -- & -- & -- & 1 & -- & -- & -- & -- & 0 & -- \\
\hline & 13006714 & -- & -- & -- & 1 & -- & -- & -- & -- & 0 & -- \\
\hline & 13009450 & -- & -- & -- & 2 & 0 & -- & -- & -- & 0 & 1 \\
\hline & 13008924 & -- & -- & -- & 1 & -- & -- & -- & -- & 0 & -- \\
\hline \multirow[t]{8}{*}{0409} & 13192736 & -- & -- & -- & 2 & -- & -- & -- & -- & 0 & -- \\
\hline & 13192794 & -- & -- & -- & 2 & -- & -- & -- & -- & 0 & -- \\
\hline & 13192762 & -- & -- & -- & 2 & -- & -- & -- & -- & 0 & -- \\
\hline & 13192474 & -- & -- & -- & 2 & 0 & -- & -- & -- & 0 & 1 \\
\hline & 13194146 & -- & -- & -- & 2 & 0 & -- & -- & -- & 0 & 1 \\
\hline & 13194090 & -- & -- & -- & 1 & -- & -- & -- & -- & 0 & -- \\
\hline & 13193728 & -- & -- & -- & 1 & 0 & -- & -- & -- & 0 & 1 \\
\hline & 13193748 & -- & -- & -- & 2 & -- & -- & -- & -- & 0 & -- \\
\hline \multirow[t]{9}{*}{0410} & 15612436 & 90060257 & 90060500 & & 1 & 0 & 90060500 & 90060257 & & 0 & 1 \\
\hline & 15613610 & -- & -- & -- & -- & 0 & -- & -- & -- & -- & 1 \\
\hline & 15644452 & -- & -- & -- & 2 & -- & -- & -- & -- & 0 & -- \\
\hline & 15627609 & -- & -- & -- & 1 & 0 & -- & -- & -- & 0 & 1 \\
\hline & 15627613 & -- & -- & -- & 2 & 0 & -- & -- & -- & 0 & 1 \\
\hline & 15636243 & -- & -- & -- & 2 & 0 & -- & -- & -- & 0 & 1 \\
\hline & 15627291 & -- & -- & -- & 1 & -- & -- & -- & -- & 0 & -- \\
\hline & 15610538 & -- & -- & -- & 2 & 0 & -- & -- & -- & 0 & 1 \\
\hline & 15605172 & -- & -- & -- & 1 & -- & -- & -- & -- & 0 & -- \\
\hline
\end{tabular}


Table 1-2. Description of classes in the 2006 National Land Cover Database.

\begin{tabular}{|c|c|c|}
\hline Class & Code & Description \\
\hline Open water & 11 & areas of open water, generally with less than 25 percent cover of vegetation or soil \\
\hline Perennial ice/snow & 12 & $\begin{array}{l}\text { areas characterized by a perennial cover of ice and (or) snow, generally greater than } 25 \text { percent of } \\
\text { total cover }\end{array}$ \\
\hline Developed-open space & 21 & $\begin{array}{l}\text { areas with a mixture of some constructed materials, but mostly vegetation in the form of lawn } \\
\text { grasses. Impervious surfaces account for less than } 20 \text { percent of total cover. These areas most } \\
\text { commonly include large-lot single-family housing units, parks, golf courses, and vegetation } \\
\text { planted in developed settings for recreation, erosion control, or aesthetic purposes. }\end{array}$ \\
\hline Developed-low intensity & 22 & $\begin{array}{l}\text { areas with a mixture of constructed materials and vegetation. Impervious surfaces account for } 20 \\
\text { to } 49 \text { percent of total cover. These areas most commonly include single-family housing units. }\end{array}$ \\
\hline Developed-medium intensity & 23 & $\begin{array}{l}\text { areas with a mixture of constructed materials and vegetation. Impervious surfaces account for } 50 \text { to } \\
79 \text { percent of the total cover. These areas most commonly include single-family housing units. }\end{array}$ \\
\hline Developed-high intensity & 24 & $\begin{array}{l}\text { highly developed areas where people reside or work in high numbers. Examples include apartment } \\
\text { complexes, row houses, and commercial/industrial. Impervious surfaces account for } 80 \text { to } \\
100 \text { percent of the total cover. }\end{array}$ \\
\hline Deciduous forest & 41 & $\begin{array}{l}\text { areas dominated by trees generally greater than } 5 \text { meters tall, and greater than } 20 \text { percent of total } \\
\text { vegetation cover. More than } 75 \text { percent of the tree species shed foliage simultaneously in } \\
\text { response to seasonal change. }\end{array}$ \\
\hline Evergreen forest & 42 & $\begin{array}{l}\text { areas dominated by trees generally greater than } 5 \text { meters tall, and greater than } 20 \text { percent of total } \\
\text { vegetation cover. More than } 75 \text { percent of the tree species maintain their leaves all year. } \\
\text { Canopy is never without green foliage. }\end{array}$ \\
\hline Mixed forest & 43 & $\begin{array}{l}\text { areas dominated by trees generally greater than } 5 \text { meters tall, and greater than } 20 \text { percent of total } \\
\text { vegetation cover. Neither deciduous nor evergreen species are greater than } 75 \text { percent of total } \\
\text { tree cover. }\end{array}$ \\
\hline Cultivated cropland & 82 & $\begin{array}{l}\text { areas used for the production of annual crops, such as corn, soybeans, vegetables, tobacco, and cot- } \\
\text { ton, and also perennial woody crops such as orchards and vineyards. Crop vegetation accounts } \\
\text { for greater than } 20 \text { percent of total vegetation. This class also includes all land being actively } \\
\text { tilled. }\end{array}$ \\
\hline Woody wetlands & 90 & $\begin{array}{l}\text { areas where forest or shrubland vegetation accounts for greater than } 20 \text { percent of vegetative cover } \\
\text { and the soil or substrate is periodically saturated with or covered with water }\end{array}$ \\
\hline Herbaceous wetlands & 95 & $\begin{array}{l}\text { areas where perennial herbaceous vegetation accounts for greater than } 80 \text { percent of vegetative } \\
\text { cover and the soil or substrate is periodically saturated with or covered with water }\end{array}$ \\
\hline
\end{tabular}


Table 1-3. Description of soil groups in the Soil Survey Geography (SSURGO) database.

Group
A $\begin{gathered}\text { Soils having a high infiltration rate (low runoff potential) when thoroughly wet. These consist mainly of deep, well drained to } \\ \text { excessively drained sands or gravelly sands. These soils have a high rate of water transmission. }\end{gathered}$
B $\begin{aligned} & \text { Soils having a moderate infiltration rate when thoroughly wet. These consist chiefly of moderately deep or deep, moderately } \\ & \text { well drained or well drained soils that have moderately fine texture to moderately coarse texture. These soils have a moder- } \\ & \text { ate rate of water transmission. }\end{aligned}$
C Soils having a slow infiltration rate when thoroughly wet. These consist chiefly of soils having a layer that impedes the down-
ward movement of water or soils of moderately fine texture or fine texture. These soils have a slow rate of water transmis-
sion.
Soils having a very slow infiltration rate (high runoff potential) when thoroughly wet. These consist chiefly of clays that have
a high shrink-swell potential, soils that have a high water table, soils that have a claypan or clay layer at or near the surface,
and soils that are shallow over nearly impervious material. These soils have a very slow rate of water transmission.

Table 1-4. Description of variables from Aquatic Gap Analysis Program (GAP) used in the analysis.

\begin{tabular}{ccl}
\hline Code & Group & \multicolumn{1}{c}{ Landform (texture) } \\
\hline \multicolumn{2}{c}{ Surficial geology } \\
\hline 2 & A3 & outwash (coarse) \\
3 & B3 & ice-contact (coarse) \\
4 & C1 & end-moraine (fine) \\
5 & C2 & end-moraine (medium) \\
6 & C3 & end-moraine (coarse) \\
7 & D1 & ground-moraine (fine) \\
8 & D2 & ground-moraine (medium) \\
& D3 & ground-moraine (coarse) \\
\hline \multicolumn{2}{c}{ Bedrock geology } \\
\hline
\end{tabular}



Publication services provided by the U.S. Geological Survey Science Publishing Network

\section{Columbus Publishing Service Center}

For more information concerning the research in this report contact the

Director, Michigan Water Science Center

U.S. Geological Survey

6520 Mercantile Way, Suite 5

Lansing, MI 48911-5991

http://mi.water.usgs.gov/ 
